GA-A 14328

UC-77

\title{
PROPERTIES OF UNIRRADIATED HTGR CORE SUPPORT AND PERMANENT SIDE REFLECTOR GRAPHITES: PGX, HLM, 2020, AND H-440N
}

\author{
by \\ G. B. ENGLE
}

Prepared under

Contract EY-76-C-03-0167

Project Agreement No. 17

for the San Francisco Operations Office

U.S. Energy Research and Development Administration 


\section{DISCLAIMER}

This report was prepared as an account of work sponsored by an agency of the United States Government. Neither the United States Government nor any agency Thereof, nor any of their employees, makes any warranty, express or implied, or assumes any legal liability or responsibility for the accuracy, completeness, or usefulness of any information, apparatus, product, or process disclosed, or represents that its use would not infringe privately owned rights. Reference herein to any specific commercial product, process, or service by trade name, trademark, manufacturer, or otherwise does not necessarily constitute or imply its endorsement, recommendation, or favoring by the United States Government or any agency thereof. The views and opinions of authors expressed herein do not necessarily state or reflect those of the United States Government or any agency thereof. 


\section{DISCLAIMER}

Portions of this document may be illegible in electronic image products. Images are produced from the best available original document. 


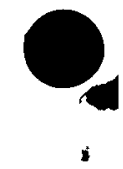

.

4 


\begin{abstract}
Candidate materials for HTGR core supports and permanent side reflectors -- graphite grades 2020 (Stackpole Carbon Company), H-440N (Great Lakes Carbon Corporation), PGX (Union Carbide Corporation), and HLM (Great Lakes Carbon Corporation) -- are described and property data are presented. Properties measured are bulk density; tensile properties including ultimate strength, modulus of elasticity, and strain at fracture; flexural strength; compressive properties including ultimate strength, modulus of elasticity, and strain at fracture; and chemical impurity content. The data presented represent the mean values and standard deviations for single logs. The logs of grades 2020, PGX, and HLM are fu11-size logs purchased from commercial stock, and the data are indicative of property values to be expected in commercial production. The data on grade $\mathrm{H}-440 \mathrm{~N}$ are from an experimental $\log$ and do not represent commercial production values.
\end{abstract}


$\bullet$ 
CONTENTS

ABSTRACT . . . . . . . . . . . . . . . . . . . . 1 . . . . . .

1. SUMMARY. . . . . . . . . . . . . . . . . . . . 1-1

1.1. Grade 2020 . . . . . . . . . . . . . . . . 1-1

1.2. Grade $\mathrm{H}-440 \mathrm{~N}$. . . . . . . . . . . . . . . . 1-2

1.3. Grade PGX. . . . . . . . . . . . . . . . . . . 1-2

1.4. Grade HLM. . . . . . . . . . . . . . . . . . . 1-2

2. INTRODUCTION . . . . . . . . . . . . . . . . . . . 2-1

3. MATERIALS. . . . . . . . . . . . . . . . . . 3-1

3.1. Core Support Posts and Seats: Grades 2020 and

$\mathrm{H}-440 \mathrm{~N}$. . . . . . . . . . . . . . . . . . 3-1

3.1.1. Grade 2020 . . . . . . . . . . . . 3-1

3.1.2. Grade $\mathrm{H}-440 \mathrm{~N}$. . . . . . . . . . . . . 3-2

3.2. Core Support Floor Blocks: Grade PGX. . . . . . . . . 3-2

3.3. Permanent Side Reflector: Grade HLM . . . . . . . . . . 3-2

4. EXPERIMENTAL METHODS . . . . . . . . . . . . . . . . 4-1

5. EXPERIMENTAL RESULTS .................... . . 5-1

5.1. Sampling . . . . . . . . . . . . . . . 5-1

5.2. Properties . . . . . . . . . . . . . . . 5-1

5.2.1. Grade 2020 . . . . . . . . . . . . . 5-2

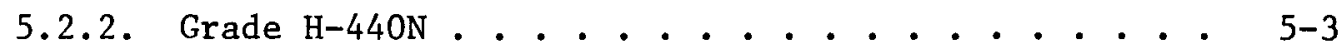

5.2.3. Grade PGX. . . . . . . . . . . . . . 5-3

5.3.4. Grade HLM. . . . . . . . . . . . . . . 5-4

6. CONCLUSIONS. . . . . . . . . . . . . . . . . . 6-1

7. ACKNOWLEDGMENTS. . . . . . . . . . . . . . . . . . 7-1

8. REFERENCES . . . . . . . . . . . . . . . . . . . 8-1

APPENDIX A: GRADE $2020 \mathrm{DATA}$. . . . . . . . . . . . . . . A-1

APPENDIX B: GRADE H-440N DATA . . . . . . . . . . . . . B-1

APPENDIX C: GRADE PGX DATA. . . . . . . . . . . . . . . C-1

APPENDIX D: GRADE HLM DATA. . . . . . . .......... D-1 


\section{FIGURES}

2-1. HTGR core and support arrangement. . . . . . . . . . . 2-3

5-1(a). Slab diagram for core support post graphite grade 2020, $\log 6484-110$................. . . 5-18

5-1(b). Core sampling plan of a slab of grade 2020, $\log 6484-110$. . . . . . . . . . . . . . . . . . 5-19

5-2(a). S1ab diagram for core support post graphite grade 2020 (fracture piece taken for test, $\log 6484-137$ ). . . . . . 5-20

5-2(b). Core sampling and specimen orientation of grade 2020, log 6484-137; axial, radial, and tangential specimens parallel to flex.............. . . 5-21

5-2(c). Core sampling and specimen orientation of grade 2020, log 6484-137; radial and tangential specimens perpendicular to flex. . . . . . . . . . . . . . . . 5-22

5-3(a). Slab diagram for core support post graphite grade 2020, $\log$ 6799-00. . . . . . . . . . . . . . . . 5-23

5-3(b). Core sampling plan of slab 1 of grade 2020, $\log 6799-00$; axial (core sampling of slabs 3 and 5 was the same as for slab 1)..................... . 5-24

5-3(c). Core sampling plan of slab 1 of grade 2020, $\log 6799-00$; radial (core sampling of slabs 3 and 5 was the same as for s1ab 1)................... 5-25

5-4. Slab diagram for core support post graphite preproduction grade $\mathrm{H}-440 \mathrm{~N}$. . . . . . . . . . . . . . . 5-26

5-5(a). Slab diagram for core support floor block and permanent side reflector block graphites (PGX, log 6484-112, or

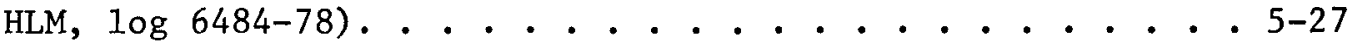

5-5(b). Sectioning of core support floor block and permanent side reflector block graphites (PGX, $\log 6484-112$, or HLM, $\log 6484-78)$. . . . . . . . . . . . . . . 5-28

5-5(c). Center section: AC. . . . . . . . . . . . . . 5-29

5-5(d). Middle section: AY. . . . . . . . . . . . 5-30

5-5(e). Edge section: AE. . . . . . . . . . . . . 5-31 
TABLES

1-1. Summary of characterization data for grade 2020, H-440N, PGX, and HLM graphites. . . . . . . . . . . . . . . 1-3

3-1. Core support and permanent side reflector graphites . . . . 3-3

5-1. Bulk density, ultimate tensile strength, and modulus of elasticity of grade 2020. . . . . . . . . . . . . 5-5

5-2. Bulk density, ultimate tensile strength, and modulus of elasticity of grade $\mathrm{H}-440 \mathrm{~N}, \log 6484-81$. . . . . . . . 5-6

5-3. Bulk density, ultimate tensile strength, and modulus of elasticity of grade PGX, $\log 6484-112$, lot 8D5-3. . . . . . 5-7

5-4. Bulk density, ultimate tensile strength, and modulus of elasticity of grade HLM, $\log 6484-78$, lot 367 . . . . . . 5-8

5-5. Flexural strength of grades 2020, H-440N, PGX, and HLM. . . 5-9

5-6. Ultimate compressive strength and modulus of elasticity of grade 2020 . . . . . . . . . . . . . . . . . 5-10

5-7. U1timate compressive strength and modulus of elasticity of grade $\mathrm{H}-440 \mathrm{~N}, \log 6484-81 . . . . . . . . . . . . .5-11$

5-8. U1timate compressive strength and modulus of elasticity of grade PGX, $\log 6484-112$, lot $85 \mathrm{D}-3$. . . . . . . . . 5-12

5-9. Ultimate compressive strength and modulus of elasticity of grade HLM, $\log 6484, \operatorname{lot} 367$. . . . . . . . . . . 5-13

5-10. Distribution of impurity content within grade 2020, $\log 6484-110,1$ ot 728 . . . . . . . . . . . . . 5-14

5-11. Distribution of impurity content within grade $\mathrm{H}-440 \mathrm{~N}$, $\log 6484-81$. . . . . . . . . . . . . . . . 5-15

5-12. Distribution of impurity content within grade PGX, $\log 6484-112$, lot 8D5-3 ............. 5-16

5-13. Distribution of impurity content within grade HLM, log 6484-78, lot $367 . . . . . . . . . .5-17$ 


\section{SUMMARY}

Three commercially available graphite grades -- Stackpole Carbon Company's 2020, Union Carbide Corporation's PGX, and Great Lakes Carbon Corporation's HLM -- were evaluated as possible candidate materials for core support posts, core support floor blocks, and permanent side reflectors, respectively, in the Large High-Temperature Gas-Cooled Reactor (LHTGR). Great Lakes Carbon Corporation's grade H-440N, an experimental material, was also evaluated as a possible candidate for core support posts. Bulk density, mechanical properties, including tensile, flexural, and compressive properties, and chemical impurities were determined as a function of location within the graphite logs. Mean values of the properties and chemical impurities of the graphites are summarized in Table $1-1 . *$

The work reported herein is part of an on-going program at General Atomic Company to select reference materials for LHTGR components.

1.1. GRADE 2020

As a candidate for core support posts, grade 2020 may be characterized as an isostatically molded, medium-grained graphite manufactured as logs $254 \mathrm{~mm}$ in diameter by $1829 \mathrm{~mm}$ in length with a bulk density of $1.79 \mathrm{Mg} / \mathrm{m}^{3}$. In comparison with other commercial graphites, it has intermediate strength and elastic moduli with a uniform distribution within a log but variation between logs. The ash content and other impurities are relatively high when compared with highly purified fuel element graphites (Ref. 1).

${ }^{*}$ Tables and figures appear at the end of each section. 


\subsection{GRADE H-440N}

As a candidate for core support posts, grade $\mathrm{H}-440 \mathrm{~N}$ may be characterized as an isostatically molded, medium-grained graphite manufactured as logs $254 \mathrm{~mm}$ in diameter by $1829 \mathrm{~mm}$ in length (the piece evaluated in this study was one-half of a prototype log manufactured with a 330 by 330 $\mathrm{mm}$ cross section and a 1829-mm length) with a bulk density of $1.72 \mathrm{Mg} / \mathrm{m}^{3}$. In comparison with other commercial graphites, it has a relatively low strength and elastic moduli that vary along the longitudinal axis. The ash content and other impurities are relatively low, approaching those of highly purified fuel element graphites (Ref. 1).

\subsection{GRADE PGX}

As a candidate for core support floor blocks, grade PGX may be characterized as a conventionally molded, medium-grained graphite manufactured as logs $1143 \mathrm{~mm}$ in diameter by $1829 \mathrm{~mm}$ in length with a bulk density of $1.78 \mathrm{Mg} / \mathrm{m}^{3}$. In comparison with other commercial graphites, it has a relatively low strength and elastic moduli which are uniformly distributed within the log. The ash content and other impurities, especially iron and sulfur, are high in comparison with those of highly purified fuel element graphites (Ref. 1).

\subsection{GRADE HLM}

As a candidate for permanent side reflector blocks, grade HLM may be characterized as an extruded, medium-grained graphite manufactured as logs $1143 \mathrm{~mm}$ in diameter by $1829 \mathrm{~mm}$ in length with a bulk density of $1.79 \mathrm{Mg} / \mathrm{m}^{3}$. In comparison with other commercial graphites, it has a relatively low strength and elastic moduli, with the weakest region at the radial center of a log. The ash content and other impurities are relatively high in comparison with those of highly purified fuel element graphites (Ref. 1). 
TABLE $1-1$

SUMMARY OF CHARACTERIZATION DATA FOR GRADE 2020, H-440N, PGX, AND HLM GRAPHITES

\begin{tabular}{|c|c|c|c|c|c|c|c|c|}
\hline \multirow[b]{3}{*}{ Property } & \multicolumn{8}{|c|}{ Graphite Grade } \\
\hline & \multicolumn{2}{|c|}{2020} & \multicolumn{2}{|c|}{$\mathrm{H}-440 \mathrm{~N}$} & \multicolumn{2}{|c|}{$\mathrm{PGX}$} & \multicolumn{2}{|c|}{ HLM } \\
\hline & Axial & Radial & Axial & Radial & Axial & Radial & Axial & Radial \\
\hline $\begin{array}{l}\text { Bulk density }\left(\mathrm{Mg} / \mathrm{m}^{3}\right) \\
\text { Tensile properties }\end{array}$ & 1.79 & -- & 1.72 & -- & 1.78 & -- & 1.79 & -- \\
\hline Strength (MPa) & 16.0 & 17.5 & 11.0 & 11.5 & 10.3 & 9.5 & 10.0 & 11.5 \\
\hline Modulus (GPa) & 7.5 & 8.5 & 6.5 & 7.5 & 6.0 & 7.0 & 7.0 & 6.0 \\
\hline $\begin{array}{l}\text { Flexural strength } \\
(\mathrm{MPa})\end{array}$ & 25.0 & 27.5 & 19.5 & 21.0 & 15.5 & 15.0 & 18.0 & 17.5 \\
\hline Compressive properties & & & & & & & & \\
\hline Strength (MPa) & 80.5 & 80.5 & 51.0 & 45.0 & 43.0 & 41.0 & 39.0 & 42.0 \\
\hline Modulus (GPa) & 9.5 & 10.0 & 5.5 & 6.0 & 5.0 & 5.5 & 6.0 & 6.0 \\
\hline Impurity content (ppm) & & & & & & & & \\
\hline Ash & 1550 & -- & 600 & -- & 6183 & -- & 2398 & -- \\
\hline $\mathrm{Si}$ & 15 & -- & 19 & -- & 541 & -- & 32 & -- \\
\hline $\mathrm{Fe}$ & 220 & -- & 14 & -- & $>1000$ & -- & $>1000$ & -- \\
\hline
\end{tabular}




\section{INTRODUCTION}

The LHTGR design utilizes graphite for core support posts, seats, and sleeves, core support and side reflector floor blocks, and permanent side reflectors. Figure 2-1 is a diagram of an HTGR core and support arrangement showing these components.

The core support posts are cylindrical columns with hemispherical ends. Posts are provided to support each core support block. The positions of the posts are fixed by the post seats, which are located in the core support blocks and on the bottom pad of the prestressed concrete reactor vessel (PCRV). The posts are free to pivot so as to follow changes in the position of the core support block. Each post supports a portion of the total weight of a multiple-column fuel assembly, plus the additional loads imposed by the drop in coolant pressure through the core.

The upper seats for the core support posts are provided as a separate insert in the bottom surface of the support blocks. The seats have one concave surface machined to a slightly larger radius than the end of the core support posts. The main functions of the seats are to permit the upper end of the post to follow any horizontal movement of the core support block and to help distribute loads at the contact surface. At the lower end of each post, a similar seat is part of an assembly which is located on the bottom pad. The lower seat is in contact with materials, such as alumina or silica, which form other parts of the assembly.

The core support and side reflector floor blocks are of a prismatic shape. Each block supports a multiple-column assembly of fuel element, hexagonal reflector, or side reflector blocks and is supported by core support posts. The blocks contain channels for the passage of coolant gas and contain separate inserts as seats for the core support posts. 
Each of the core support blocks is closely assembled to the adjacent blocks, and the outermost blocks are keyed to the lateral restraint structure. The core support blocks are designed to fix the position of the fuel columns at the bottom of the reactor core.

The permanent side reflectors consist of large blocks which encircle the core and are keyed to the core lateral restraint structure, which is attached to the PCRV.

The primary function of the permanent reflector blocks is to moderate the fast-neutron flux in the reflector region and reflect thermal neutrons back into the core region, thus helping to reduce the neutron flux at the thermal barrier and the PCRV liner. In addition, the permanent reflector blocks form an intermediate support between the PCRV and the keyed plenum elements to help restrict horizontal movement of the core support blocks.

This report describes the initial results of property measurements on three commercial graphite grades which may be utilized for the abovementioned components: grade 2020, manufactured by Stackpole Carbon Company (SC), for support posts; grade PGX, manufactured by Union Carbide Corporation (UCC), for core support floor blocks; and grade HLM, manufactured by Great Lakes Carbon Corporation (GLCC) for permanent side reflector blocks. These grades are available as commercial products from U.S. graphite manufacturers and are manufactured in sizes that satisfy the LHTGR design.

Data are presented for one log each of grades 2020, PGX, and HLM and a portion of a $\mathrm{log}$ of an experimental grade, H-440N, manufactured by GLCC. The purpose of this work is to characterize the materials with regard to properties and chemical impurity content. 


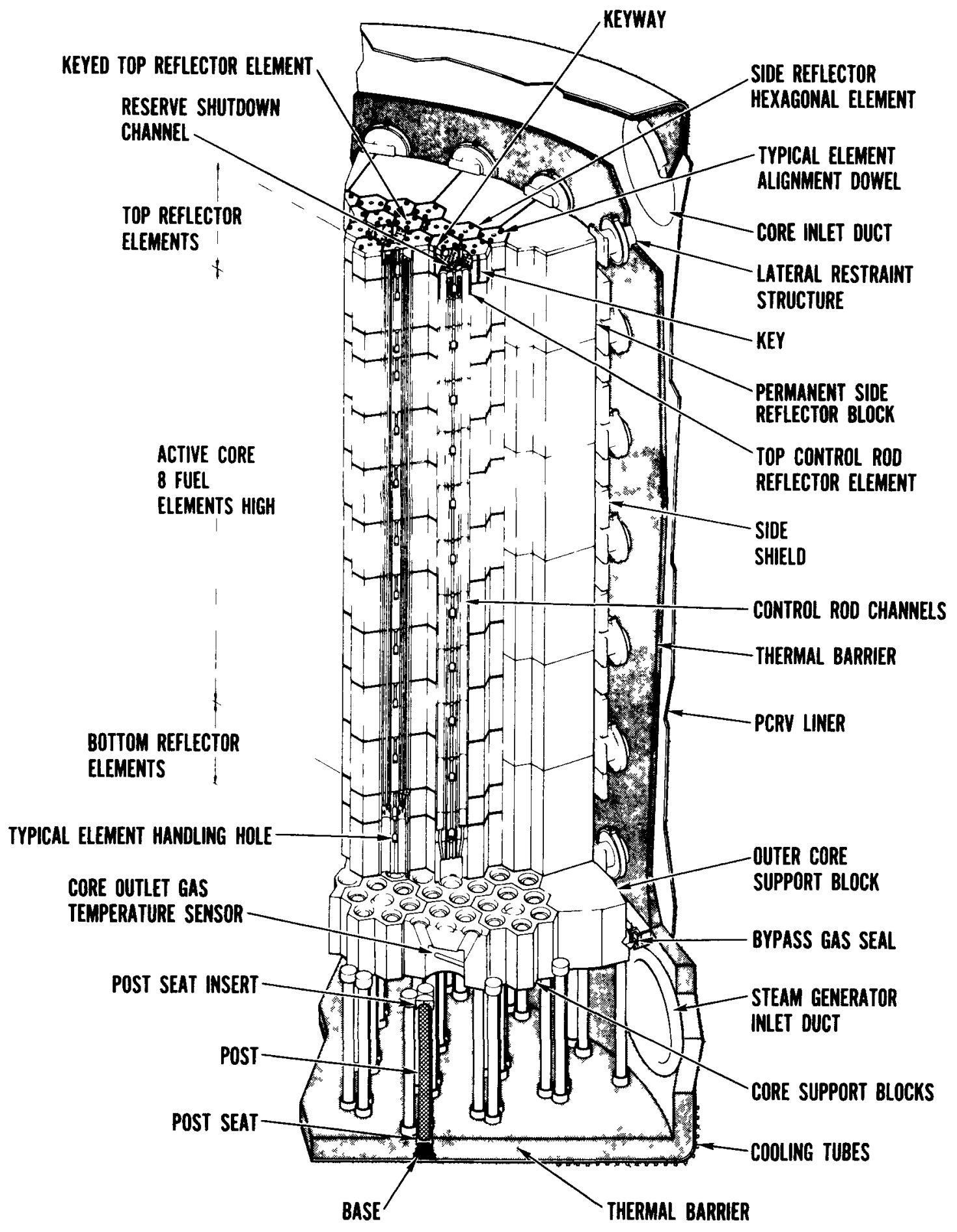

Fig. 2-1. HTGR core and support arrangement 


\section{MATERIALS}

The graphites described in this section are produced by major U.S. graphite companies. The raw materials and details of their processing are considered proprietary by the respective manufacturers. However, all grades were fabricated by conventional graphite methods consisting of mixing filler particles of petroleum coke or recycled graphite with a coal tar pitch binder, forming by molding or extrusion, baking to carbonize the binder, with possible impregnation with coa1-tar pitch or petroleum pitch to improve the properties, and graphitizing in the range 2873 to $3173 \mathrm{~K}\left(2600^{\circ}\right.$ to $\left.2900^{\circ} \mathrm{C}\right)$.

A description of the graphites is given in Table 3-1.

3.1. CORE SUPPORT POSTS AND SEATS: GRADES 2020 AND H-440N

3.1.1. Grade 2020

Grade 2020 is manufactured by the Stackpole Carbon Company in a variety of sizes for commercial sales. Grade 2020 is formed by isostatic molding followed by conventional baking and graphitizing processes. The product being evaluated in this program is manufactured as $\log s 254 \mathrm{~mm}$ in diameter by $1829 \mathrm{~mm}$ in length using petroleum coke as a filler and coal tar pitch as a binder. The logs tested were purchased at different times (see Table 5-1) and thus represent logs manufactured at different times in the Stackpole plant. Log 6484-110 was tested under a characterization program and was systematically sampled; $\log 6484-137$ was compression tested as a fullsize $\log$, and a piece that broke out of the $\log$ as a result of the test was sampled for testing (see Fig. 5-3); and $\log$ 6799-00 was utilized in another study to determine the effect of oxidation on strength. The data from the control specimens of the oxidation study are included in this report. 


\subsubsection{Grade $\mathrm{H}-440 \mathrm{~N}$}

Grade $\mathrm{H}-440 \mathrm{~N}$ is a preproduction material manufactured by the Great Lakes Carbon Corporation. GLCC is in the process of commercializing grade $\mathrm{H}-440 \mathrm{~N}$, and the $\log$ that was evaluated in this program was formed by isostatic molding. It had a $330-\mathrm{mm}$ by $330-\mathrm{mm}$ cross section and a $1829-\mathrm{mm}$ length and was manufactured with petroleum coke as filler and coal tar pitch as binder. One-half of the $\log$ was examined.

\subsection{CORE SUPPORT FLOOR BLOCKS: GRADE PGX}

Grade PGX is manufactured by the Union Carbide Corporation for commercial sales. Grade PGX is formed by conventional molding followed by conventional baking and graphitizing processes. The product being evaluated in this program was manufactured as a $\log 1143 \mathrm{~mm}$ in diameter by 1829 $\mathrm{mm}$ long using recycled graphite as filler and coal tar pitch as binder.

\subsection{PERMANENT SIDE REFLECTOR: GRADE HLM}

Grade HLM is manufactured by the Great Lakes Carbon Corporation for commercial sales. Grade HLM is formed by conventional extrusion followed by conventional baking and graphitizing processes. The HLM material eva1uated in this program was manufactured as a $\log 1143 \mathrm{~mm}$ in diameter by $1829 \mathrm{~mm}$ long using calcined petroleum coke and recycle graphite as filler and coal tar pitch as binder. 
TABLE 3-1

CORE SUPPORT AND PERMANENT SIDE REFLECTOR GRAPHITES

\begin{tabular}{|c|c|c|c|c|c|}
\hline Grade & Manufacturer & $\begin{array}{l}\text { Reactor } \\
\text { Component }\end{array}$ & Filler & Binder & $\begin{array}{l}\text { Forming } \\
\text { Method }\end{array}$ \\
\hline 2020 & $\mathrm{SC}$ & $\begin{array}{l}\text { Core support } \\
\text { posts }\end{array}$ & $\begin{array}{l}\text { Petroleum } \\
\text { coke }\end{array}$ & $\begin{array}{l}\text { Coal tar } \\
\text { pitch }\end{array}$ & $\begin{array}{l}\text { Isostatic } \\
\text { molding }\end{array}$ \\
\hline PGX & UCC & $\begin{array}{l}\text { Core support } \\
\text { floor }\end{array}$ & $\begin{array}{l}\text { Recycle } \\
\text { graphite }\end{array}$ & $\begin{array}{l}\text { Coal tar } \\
\text { pitch }\end{array}$ & $\begin{array}{l}\text { Conventional } \\
\text { molding }\end{array}$ \\
\hline HLM & GLCC & $\begin{array}{l}\text { Permanent side } \\
\text { reflector }\end{array}$ & $\begin{array}{l}\text { Pet. coke + } \\
\text { recycle } \\
\text { graphite }\end{array}$ & $\begin{array}{l}\text { Coal tar } \\
\text { pitch }\end{array}$ & Extrusion \\
\hline $\mathrm{H}-440 \mathrm{~N}^{(\mathrm{a})}$ & GLCC & $\begin{array}{l}\text { Core support } \\
\text { posts }\end{array}$ & $\begin{array}{l}\text { Petroleum } \\
\text { coke }\end{array}$ & $\begin{array}{l}\text { Coal tar } \\
\text { pitch }\end{array}$ & $\begin{array}{l}\text { Isostatic } \\
\text { molding }\end{array}$ \\
\hline
\end{tabular}

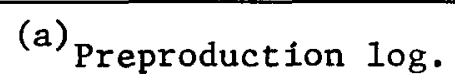


4. EXPERIMENTAL METHODS

Details of the experimental methods are given in Ref. 1.

Compressive properties were measured in accordance with ASTM C695, "Compressive Crushing Strength of Graphite," 1976 Annual Book of ASTM Standards, Part 17 . 


\section{EXPERIMENTAL RESULTS}

\subsection{SAMPLING}

Commercial graphite logs of the size used for core support posts and core support floor blocks and permanent side reflector blocks have been shown to have nonuniform distributions of properties within a single $10 \mathrm{~g}$ and from $\log$ to $\log$ within a manufactured lot (Refs. 1-6). Therefore, properties and chemical impurity contents were measured as a function of location within each log. Mechanical properties were measured on specimens taken parallel (axial) and perpendicular (radial) to the longitudinal axis of the parent $\operatorname{logs}$ and at several locations in each $\log$ as shown in Figs. 5-1 through 5-5.

Only one-half of the H-440N and HLM logs was tested, whereas fu11 logs of grades 2020 and PGX were used.

\subsection{PROPERTIES}

Complete data sets of the graphite properties are given in Appendices $A, B, C$, and D. The data are presented in summary form in this section.

The following properties were measured on grades 2020, PGX, HLM, and H-440N: (1) bulk density, tensile properties including ultimate tensile strength, strain at fracture, and modulus of elasticity; (2) flexural strength; (3) compressive properties including ultimate compressive strength, strain at fracture, and modulus of elasticity; and (4) chemical impurity content. Thermal expansivity and thermal conductivity data will be reported later.

The physical, mechanical, and chemical property data are presented in Tables 5-1 through 5-13. The various graphite grades being evaluated in 
this characterization program are generically similar; i.e., they are manufactured from the same basic raw materials and processes. However, they are different in detail (see Table 3-1). The various grades for each reactor component are manufactured in different sizes and by different manufacturers, and therefore each grade is expected to have unique properties. Gradients within logs were also expected depending upon the raw materials, processing, and $\log$ size. Thus, the data are presented as mean values of specimens taken from various regions of each log. A detailed inspection of data in the summary tables will reveal the axial and radia1 property gradients characteristic of each grade. The data in Tables 5-1 and 5-9 reveal differences in bulk density, strength, and elastic modulus among logs of grade 2020 that were purchased at different times from Stackpole Carbon Company. The data for grades H-44ON, PGX, and HLM are confined to a single $\log$.

\subsubsection{Grade 2020}

The mechanical property data for grade 2020 are given in Tables 5-1, 5-5, and 5-6 and the chemical impurity content data in Table 5-10. The strengths and elastic modulus values varied among the three logs tested. Log 6799-00, purchased in 1974, was significantly stronger than the log purchased in 1976. However, differences in strength along the longitudinal and radial axes were not excessive. The scatter among specimens at a single location in a $\log$ was not excessive. A summary of the tensile strength and elastic modulus values for each $\log$ is given below:

\begin{tabular}{l|c|c|c|c}
\hline \multirow{2}{*}{$\begin{array}{l}\text { Log } \\
\text { No. }\end{array}$} & \multicolumn{2}{|c|}{ UTS (MPa) } & \multicolumn{2}{c}{ E (GPa) } \\
\cline { 2 - 5 } & Axial & Radial & Axial & Radial \\
\hline $6484-110$ & 14.9 & 17.4 & 7.2 & 7.8 \\
$6484-137$ & 15.5 & 17.0 & 8.1 & 9.4 \\
$6799-00$ & 18.0 & 18.8 & -- & -- \\
\hline
\end{tabular}


The ash content was higher at the end of $\log 6484-110$ than at the middle by a factor of 1.7 (1900 ppm and $1100 \mathrm{ppm}$, respectively). This gradient was also reflected in the iron and silicon contents, but was reversed for titanium.

\subsubsection{Grade $\mathrm{H}-440 \mathrm{~N}$}

The mechanical property data for grade $\mathrm{H}-440 \mathrm{~N}$ are given in Tables 5-2, 5-5, and 5-7 and the chemical impurity content data in Table 5-11. The strengths and elastic modulus were higher at the end of the log than at the middle. The overall strength of grade $\mathrm{H}-440 \mathrm{~N}$ was only about $75 \%$ of the strength of the weakest grade $2020 \mathrm{log}$.

The ash content of $\log 6484-81$ averaged about $600 \mathrm{ppm}$, and other impurity elements were relatively low. There were no gradients within the log. Grade H-440N is much purer than grade 2020, approaching but not equaling highly purified fuel element graphites (Ref. 1).

\subsubsection{Grade PGX}

Mechanical property data for grade PGX are given in Tables 5-3, 5-5, and 5-8 and chemical impurity content data in Table 5-12. Strength and elastic moduli were quite uniform along the longitudinal and radial axes of $\log 6484-112$, with the bottom of the $\log$ slightly weaker than the top and middle. A summary of the mean tensile strength and elastic modulus values for each slab is given below:

\begin{tabular}{l|c|c|c|c}
\hline \multirow{2}{*}{ Slab } & \multicolumn{2}{|c|}{ UTS (MPa) } & \multicolumn{2}{c}{ E (GPa) } \\
\cline { 2 - 5 } End-1 & Axial & Radial & Axial & Radial \\
Middle-6 & 11.0 & 10.2 & 6.3 & 6.9 \\
Bottom-12 & 10.8 & 10.2 & 6.4 & 7.4 \\
& 9.2 & 8.6 & 6.1 & 7.1 \\
\hline
\end{tabular}


The ash content was high, ranging from about 800 to $11,000 \mathrm{ppm}$. Other impurity elements, such as iron, calcium, and sulfur, were also high. The middle region of the $\log$ was purer than the top or bottom.

\subsubsection{Grade HLM}

Mechanical property data for grade HLM are given in Tables 5-4, 5-5, and 5-9 and the chemical impurity content data in Table 5-13. The axial tensile strengths and modulus values and the axial flexural strengths were highest at the edge of the $\log$, whereas the radial strength and modulus values and the compressive properties were more uniform. A summary of the mean tensile strength and modulus values for each slab is given below:

\begin{tabular}{c|c|c|c|c}
\hline \multirow{2}{*}{ Slab } & \multicolumn{2}{|c|}{ UTS (MPa) } & \multicolumn{2}{c}{ E (GPa) } \\
\cline { 2 - 5 } & Axia1 & Radial & Axia1 & Radia1 \\
\hline \multirow{2}{*}{ End-1 } & 10.3 & 11.9 & 7.1 & 6.2 \\
Middle-6 & 9.8 & 11.0 & 7.1 & 6.3 \\
\hline
\end{tabular}

The ash content of grade HLM ranged from about 700 to $4900 \mathrm{ppm}$. The iron content was relatively high, being somewhat higher at the end of the log than in the middle. 
TABLE 5-1

BULK DENSITY, ULTIMATE TENSILE STRENGTH, AND MODULUS OF ELASTICITY OF GRADE 2020

\begin{tabular}{|c|c|c|c|c|c|c|c|c|c|c|c|c|c|}
\hline \multirow{2}{*}{$\begin{array}{l}\text { Log } \\
\text { Number }\end{array}$} & \multirow{2}{*}{$\begin{array}{l}\text { Lot } \\
\text { No. }\end{array}$} & \multirow{2}{*}{$\begin{array}{c}\text { Date } \\
\text { Received }\end{array}$} & \multirow{2}{*}{$\begin{array}{c}\text { Location } \\
\text { in Log }\end{array}$} & \multirow{2}{*}{$\begin{array}{l}\text { Orientation } \\
\text { of Specimens }\end{array}$} & \multicolumn{3}{|c|}{$\begin{array}{c}\text { Mean Density } \\
\left(\mathrm{Mg} / \mathrm{m}^{3}\right)\end{array}$} & \multicolumn{3}{|c|}{$\begin{array}{c}\text { Mean Tensile } \\
\text { Strength } \\
(\mathrm{MPa})\end{array}$} & \multicolumn{3}{|c|}{$\begin{array}{c}\text { Mean Elastic } \\
\text { Modulus } \\
(\mathrm{GPa})\end{array}$} \\
\hline & & & & & $\bar{\rho}$ & $\sigma$ & $\mathrm{N}^{(a)}$ & $\bar{S}$ & $\sigma$ & $\mathrm{N}(\mathrm{a})$ & $\overline{\mathrm{E}}$ & $\sigma$ & $\mathrm{N}(\mathrm{a})$ \\
\hline \multirow[t]{4}{*}{$6484-110$} & \multirow[t]{4}{*}{728} & \multirow[t]{4}{*}{ May 1976} & End slab & Axia1 & 1.787 & 0.006 & 12 & 14.1 & 1.9 & 36 & 7.3 & 0.2 & 12 \\
\hline & & & 1(b) & Radial & 1.789 & 0.002 & 8 & 15.8 & 1.4 & 20 & 7.9 & 0.2 & 8 \\
\hline & & & Middle & Axia1 & 1.790 & 0.009 & 12 & 15.8 & 2.4 & 36 & 7.1 & 0.2 & 12 \\
\hline & & & slab 6 & Radial & 1.796 & 0.002 & 8 & 18.9 & 1.5 & 20 & 7.6 & 0.1 & 8 \\
\hline \multirow{5}{*}{$6484-137$} & \multirow{3}{*}{$\begin{array}{l}-- \\
-- \\
--\end{array}$} & \multirow[t]{5}{*}{ Oct. 1974} & \multirow{3}{*}{$\left.\begin{array}{l}\text { Middle } \\
\text { slab } 1(\mathrm{c})\end{array}\right\}$} & Axial & -- & -- & -- & 15.5 & 1.2 & 10 & 8.1 & 0.1 & 4 \\
\hline & & & & Radial & -- & -- & -- & 16.4 & 1.5 & 10 & 9.3 & 0.1 & 4 \\
\hline & & & & Tangential & -- & -- & -- & 20.2 & 1.6 & 10 & 9.5 & 0.2 & 4 \\
\hline & -- & & Middle & Radial & -- & -- & - & 17.6 & 2.0 & 10 & 9.5 & 0.1 & 4 \\
\hline & -- & & slab 2 & Tangential & -- & -- & -- & 14.6 & 1.4 & 10 & 10.1 & 0.2 & 4 \\
\hline \multirow{6}{*}{ 6799-00 } & -- & \multirow[t]{6}{*}{ Dec. 1974} & End slab & Axial & 1.784 & 0.004 & 8 & 18.5 & 1.1 & 8 & -- & -- & -- \\
\hline & -- & & $1(\mathrm{~d}) \quad\}$ & Radial & 1.780 & 0.003 & 8 & 18.8 & 1.7 & 8 & -- & -- & -- \\
\hline & -- & & Middle & Axial & 1.789 & 0.002 & 8 & 17.4 & 0.6 & 8 & -- & -- & -- \\
\hline & -- & & slab 3 & Radial & 1.783 & 0.003 & 8 & 18.6 & 1.1 & 8 & -- & -- & -- \\
\hline & -- & & End slab & Axial & 1.799 & 0.005 & 8 & 18.2 & 1.2 & 8 & -- & -- & -- \\
\hline & -- & & $5 \quad f$ & Radial & 1.805 & 0.004 & 8 & 19.1 & 1.1 & 8 & $-\infty$ & -- & -- \\
\hline
\end{tabular}

(a) $\mathrm{N}=$ number of replicates tested.

(b) See Fig. 5-1 for location in log.

(c) See Fig. 5-2 for location in $10 \mathrm{~g}$.

(d) See Fig. 5-3 for location in 10 . 
TABLE 5-2

BULK DENSITY, ULTIMATE TENSILE STRENGTH, AND MODULUS OF ELASTICITY OF GRADE H-440N, LOG 6484-81

\begin{tabular}{|c|c|c|c|c|c|c|c|c|c|c|}
\hline \multirow{2}{*}{$\begin{array}{l}\text { Location } \\
\text { in } \log (a)\end{array}$} & \multirow{2}{*}{$\begin{array}{l}\text { Orientation } \\
\text { of Specimens }\end{array}$} & \multicolumn{3}{|c|}{$\begin{array}{l}\text { Mean Density } \\
\left(\mathrm{Mg} / \mathrm{m}^{3}\right)\end{array}$} & \multicolumn{3}{|c|}{$\begin{array}{c}\text { Mean Tensile } \\
\text { Strength } \\
(\mathrm{MPa})\end{array}$} & \multicolumn{3}{|c|}{$\begin{array}{l}\text { Mean Elastic } \\
\text { Modulus } \\
(\mathrm{GPa})\end{array}$} \\
\hline & & $\bar{\rho}$ & $\sigma$ & $\mathrm{N}^{(\mathrm{b})}$ & $\overline{\bar{S}}$ & $\sigma$ & $N^{(b)}$ & $\overline{\mathrm{E}}$ & $\sigma$ & $\mathrm{N}^{(\mathrm{b})}$ \\
\hline End slab 1 & Axial & 1.746 & 0.004 & 12 & 11.2 & 1.5 & 34 & 7.1 & 0.3 & 12 \\
\hline End slab 1 & Radial & 1.740 & 0.007 & 8 & 13.1 & 1.1 & 20 & 8.0 & 0.7 & 8 \\
\hline Middle slab 6 & Axial & 1.706 & 0.029 & 12 & 10.9 & 1.3 & 34 & 6.0 & 1.0 & 12 \\
\hline Middle slab 6 & Radial & 1.700 & 0.018 & 8 & 10.4 & 1.3 & 20 & 7.0 & 0.8 & 8 \\
\hline
\end{tabular}

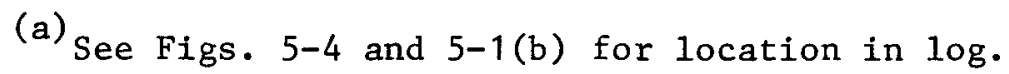

${ }^{(b)}{ }_{N}=$ number of replicates tested. 
TABLE 5-3

BULK DENSITY, ULTIMATE TENSILE STRENGTH, AND MODULUS OF ELASTICITY OF GRADE PGX, LOG 6484-112, LOT 8D5-3

\begin{tabular}{|c|c|c|c|c|c|c|c|c|c|c|c|}
\hline \multicolumn{2}{|c|}{ Location in Log (a) } & \multirow{2}{*}{$\begin{array}{l}\text { Orientation } \\
\text { of Specimens }\end{array}$} & \multicolumn{3}{|c|}{$\begin{array}{l}\text { Mean Density } \\
\left(\mathrm{Mg} / \mathrm{m}^{3}\right)\end{array}$} & \multicolumn{3}{|c|}{$\begin{array}{c}\text { Mean Tensile } \\
\text { Strength } \\
(\mathrm{MPa})\end{array}$} & \multicolumn{3}{|c|}{$\begin{array}{l}\text { Mean Elastic } \\
\text { Modulus } \\
(\mathrm{GPa})\end{array}$} \\
\hline Slab & Section & & $\bar{\rho}$ & $\sigma$ & $\mathrm{N}^{(\mathrm{b})}$ & $\overline{\mathrm{S}}$ & $\sigma$ & $\mathrm{N}^{(\mathrm{b})}$ & $\bar{E}$ & $\sigma$ & $\mathrm{N}^{(\mathrm{b})}$ \\
\hline End-1 & $\begin{array}{l}\mathrm{AC}-\mathrm{BC} \\
\mathrm{AC}-\mathrm{BC} \\
\mathrm{AY}-\mathrm{BY} \\
\mathrm{AY}-\mathrm{BY} \\
\mathrm{AE}-\mathrm{BE} \\
\mathrm{AE}-\mathrm{BE}\end{array}$ & $\begin{array}{l}\text { Axial } \\
\text { Radial } \\
\text { Axial } \\
\text { Radial } \\
\text { Axial } \\
\text { Radial }\end{array}$ & $\begin{array}{l}1.781 \\
1.776 \\
1.774 \\
1.774 \\
1.790 \\
1.790\end{array}$ & $\begin{array}{l}0.005 \\
0.006 \\
0.003 \\
0.002 \\
0.016 \\
0.002\end{array}$ & $\begin{array}{l}8 \\
8 \\
8 \\
8 \\
8 \\
8\end{array}$ & $\begin{array}{r}10.5 \\
9.6 \\
11.1 \\
10.2 \\
11.4 \\
10.9\end{array}$ & $\begin{array}{l}2.0 \\
0.7 \\
0.5 \\
0.8 \\
0.6 \\
0.6\end{array}$ & $\begin{array}{l}24 \\
20 \\
24 \\
20 \\
24 \\
20\end{array}$ & $\begin{array}{l}6.5 \\
6.7 \\
6.2 \\
6.8 \\
6.3 \\
7.2\end{array}$ & $\begin{array}{l}0.3 \\
0.2 \\
0.2 \\
0.3 \\
0.2 \\
0.2\end{array}$ & $\begin{array}{l}7 \\
8 \\
8 \\
8 \\
8 \\
8\end{array}$ \\
\hline Middle-6 & $\begin{array}{l}A C-B C \\
A C-B C \\
A Y-B Y \\
A Y-B Y \\
A E-B E \\
A E-B E\end{array}$ & $\begin{array}{l}\text { Axial } \\
\text { Radial } \\
\text { Axial } \\
\text { Radial } \\
\text { Axial } \\
\text { Radial }\end{array}$ & $\begin{array}{l}1.779 \\
1.778 \\
1.780 \\
1.783 \\
1.790 \\
1.791\end{array}$ & $\begin{array}{l}0.008 \\
0.005 \\
0.003 \\
0.004 \\
0.004 \\
0.002\end{array}$ & $\begin{array}{l}8 \\
8 \\
8 \\
8 \\
8 \\
8\end{array}$ & $\begin{array}{r}10.0 \\
9.2 \\
10.9 \\
10.3 \\
11.4 \\
11.0\end{array}$ & $\begin{array}{l}0.7 \\
0.8 \\
0.7 \\
0.7 \\
0.6 \\
0.9\end{array}$ & $\begin{array}{l}24 \\
20 \\
24 \\
20 \\
24 \\
20\end{array}$ & $\begin{array}{l}6.2 \\
7.1 \\
6.5 \\
7.3 \\
6.6 \\
7.7\end{array}$ & $\begin{array}{l}0.2 \\
0.2 \\
0.2 \\
0.2 \\
0.1 \\
0.2\end{array}$ & $\begin{array}{l}8 \\
8 \\
8 \\
8 \\
8 \\
8\end{array}$ \\
\hline Bottom- 12 & $\begin{array}{l}A C-B C \\
A C-B C \\
A Y-B Y \\
A Y-B Y \\
A E-B E \\
A E-B E\end{array}$ & $\begin{array}{l}\text { Axial } \\
\text { Radial } \\
\text { Axial } \\
\text { Radial } \\
\text { Axial } \\
\text { Radial }\end{array}$ & $\begin{array}{l}1.783 \\
1.777 \\
1.773 \\
1.777 \\
1.780 \\
1.775\end{array}$ & $\begin{array}{l}0.009 \\
0.004 \\
0.005 \\
0.005 \\
0.010 \\
0.003\end{array}$ & $\begin{array}{l}8 \\
8 \\
8 \\
8 \\
8 \\
8\end{array}$ & $\begin{array}{l}9.1 \\
8.9 \\
9.1 \\
8.1 \\
9.4 \\
8.8\end{array}$ & $\begin{array}{l}0.9 \\
1.1 \\
0.6 \\
1.4 \\
0.7 \\
0.9\end{array}$ & $\begin{array}{l}24 \\
20 \\
24 \\
20 \\
24 \\
20\end{array}$ & $\begin{array}{l}6.2 \\
7.4 \\
6.0 \\
6.9 \\
6.1 \\
6.9\end{array}$ & $\begin{array}{l}0.5 \\
0.3 \\
0.2 \\
0.2 \\
0.2 \\
0.3\end{array}$ & $\begin{array}{l}8 \\
8 \\
8 \\
8 \\
8 \\
8\end{array}$ \\
\hline
\end{tabular}

(a) See Fig. 5-5 for location in $10 \mathrm{~g}$.

(b) ${ }_{N}=$ number of replicates tested. 
TABLE 5-4

BULK DENSITY, ULTIMATE TENSILE STRENGTH, AND MODULUS OF ELASTICITY OF GRADE HLM, LOG 6484-78, LOT 367

\begin{tabular}{|c|c|c|c|c|c|c|c|c|c|c|c|}
\hline \multicolumn{2}{|c|}{ Location in Log (a) } & \multirow{2}{*}{$\begin{array}{l}\text { Orientation } \\
\text { of Specimens }\end{array}$} & \multicolumn{3}{|c|}{$\begin{array}{l}\text { Mean Density } \\
\left(\mathrm{Mg} / \mathrm{m}^{3}\right)\end{array}$} & \multicolumn{3}{|c|}{$\begin{array}{c}\text { Mean Tensile } \\
\text { Strength } \\
(\mathrm{MPa})\end{array}$} & \multicolumn{3}{|c|}{$\begin{array}{l}\text { Mean Elastic } \\
\text { Modulus } \\
\text { (GPa) }\end{array}$} \\
\hline Slab & Section & & $\bar{\rho}$ & $\sigma$ & $N^{(b)}$ & $\bar{S}$ & $\sigma$ & $N^{(b)}$ & $\overline{\mathrm{E}}$ & $\sigma$ & $\mathrm{N}^{(\mathrm{b})}$ \\
\hline \multirow{6}{*}{ End-1 } & $A C-B C$ & Axial & 1.784 & 0.008 & 8 & 7.8 & 2.1 & 24 & 6.9 & 0.1 & 3 \\
\hline & $A C-B C$ & Radial & 1.780 & 0.007 & 8 & 11.9 & 1.8 & 20 & 6.6 & 0.3 & 7 \\
\hline & $A Y-B Y$ & Axial & 1.774 & 0.008 & 8 & 9.4 & 1.0 & 24 & 7.0 & 0.3 & 7 \\
\hline & $A Y-B Y$ & Radial & 1.774 & 0.007 & 8 & 12.0 & 0.7 & 20 & 6.2 & 0.2 & 8 \\
\hline & $\mathrm{AE}-\mathrm{BE}$ & Axial & 1.772 & 0.009 & 8 & 13.8 & 1.2 & 24 & 7.4 & 0.6 & 8 \\
\hline & $A E-B E$ & Radial & 1.771 & 0.008 & 8 & 11.9 & 1.1 & 20 & 5.8 & 0.4 & 7 \\
\hline \multirow{6}{*}{ Middle-6 } & $\mathrm{AC}-\mathrm{BC}$ & Axial & 1.796 & 0.006 & 8 & 6.8 & 1.0 & 24 & 6.5 & 0.5 & 5 \\
\hline & $A C-B C$ & Radial & 1.796 & 0.003 & 8 & 9.9 & 1.3 & 20 & 6.6 & 0.2 & 8 \\
\hline & $A Y-B Y$ & Axial & 1.798 & 0.005 & 8 & 9.3 & 2.2 & 24 & 7.2 & 0.8 & 4 \\
\hline & $\mathrm{AY}-\mathrm{BY}$ & Radial & 1.795 & 0.006 & 8 & 10.6 & 1.7 & 20 & 6.2 & 0.4 & 7 \\
\hline & $\mathrm{AE}-\mathrm{BE}$ & Axial & 1.794 & 0.004 & 8 & 13.3 & 1.8 & 24 & 7.7 & 0.5 & 8 \\
\hline & $\mathrm{AE}-\mathrm{BE}$ & Radial & 1.794 & 0.006 & 8 & 12.4 & 0.8 & 20 & 6.1 & 0.3 & 8 \\
\hline
\end{tabular}

(a) See Fig. 5-5 for location in log.

${ }^{(b)} \mathrm{N}=$ number of replicates tested. 
TABLE 5-5

FLEXURAL STRENGTH OF GRADES 2020, H-440N, PGX, AND HLM

\begin{tabular}{|c|c|c|c|c|c|c|c|c|}
\hline \multirow{3}{*}{$\begin{array}{l}\text { Graphite } \\
\text { Grade } \\
\end{array}$} & \multirow[b]{3}{*}{ Log No. } & \multirow{3}{*}{$\begin{array}{l}\text { Lot } \\
\text { No. }\end{array}$} & \multicolumn{2}{|c|}{ Location in Log } & \multirow{3}{*}{$\begin{array}{l}\text { Orientation } \\
\text { of Specimens }\end{array}$} & \multirow{2}{*}{\multicolumn{3}{|c|}{$\begin{array}{c}\text { Mean Flexural } \\
\text { Strength } \\
(\text { (MPa) }\end{array}$}} \\
\hline & & & & & & & & \\
\hline & & & slab & Section & & $\overline{\mathrm{S}}$ & $\sigma$ & $\mathrm{N}(\mathrm{a})$ \\
\hline 2020 & $6484-110$ & 1 & $\begin{array}{l}\text { End-1 } \\
\text { End-1 } \\
\text { Midd le-6 } \\
\text { Middle-6 }\end{array}$ & $\begin{array}{l}-{ }^{(b)} \\
--\end{array}$ & $\begin{array}{l}\text { Axial } \\
\text { Radial } \\
\text { Axial } \\
\text { Radial }\end{array}$ & $\begin{array}{l}25.0 \\
27.4 \\
25.2 \\
27.2\end{array}$ & $\begin{array}{l}2.4 \\
0.9 \\
0.8 \\
0.8\end{array}$ & $\begin{array}{l}42 \\
20 \\
42 \\
20\end{array}$ \\
\hline $\mathrm{H}-440 \mathrm{~N}$ & | & $\begin{array}{l}-- \\
-- \\
--\end{array}$ & $\begin{array}{l}\text { End-1 } \\
\text { End-1 } \\
\text { Middle-6 } \\
\text { Middle-6 }\end{array}$ & $\begin{array}{l}-(c) \\
--\end{array}$ & $\begin{array}{l}\text { Axial } \\
\text { Radial } \\
\text { Axial } \\
\text { Radial }\end{array}$ & $\begin{array}{l}20.5 \\
21.8 \\
18.5 \\
20.1\end{array}$ & $\begin{array}{l}1.1 \\
1.4 \\
1.8 \\
1.6\end{array}$ & $\begin{array}{l}42 \\
20 \\
42 \\
20\end{array}$ \\
\hline PGX & ${ }^{6484-112}$ & $8 D_{1} 5-3$ & $\begin{array}{l}\text { End-1 } \\
\text { End-1 } \\
\text { End-1 } \\
\text { End-1 } \\
\text { End-1 } \\
\text { End-1 }\end{array}$ & $\begin{array}{l}A C-B C(d) \\
A C-B C \\
A Y-B Y \\
A Y-B Y \\
A E-B E \\
A E-B E\end{array}$ & $\begin{array}{l}\text { Axial } \\
\text { Radial } \\
\text { Axial } \\
\text { Radial } \\
\text { Axial } \\
\text { Radial }\end{array}$ & $\begin{array}{l}15.8 \\
15.1 \\
16.1 \\
15.5 \\
16.0 \\
16.6\end{array}$ & $\begin{array}{l}1.2 \\
1.8 \\
1.0 \\
1.8 \\
0.9 \\
1.2\end{array}$ & $\begin{array}{l}24 \\
20 \\
20 \\
20 \\
20 \\
20\end{array}$ \\
\hline PGX & $6484-112$ & $\begin{array}{c}8 \mathrm{D} 5-3 \\
\mid\end{array}$ & $\begin{array}{l}\text { Midd le-6 } \\
\text { Midd 1e-6 } \\
\text { Midd 1e-6 } \\
\text { Midd 1e-6 } \\
\text { Midd 1e-6 } \\
\text { Midd1e-6 }\end{array}$ & $\begin{array}{l}A C-B C \\
A C-B C \\
A Y-B Y \\
A Y-B Y \\
A E-B E \\
A E-B E\end{array}$ & $\begin{array}{l}\text { Axial } \\
\text { Radial } \\
\text { Axial } \\
\text { Radial } \\
\text { Axial } \\
\text { Radial }\end{array}$ & $\begin{array}{l}15.3 \\
14.3 \\
15.9 \\
15.7 \\
16.8 \\
17.1\end{array}$ & $\begin{array}{l}0.9 \\
2.2 \\
0.8 \\
1.5 \\
0.8 \\
1.6\end{array}$ & $\begin{array}{l}24 \\
20 \\
20 \\
20 \\
20 \\
20\end{array}$ \\
\hline$\stackrel{\text { PGX }}{\mid}$ & $\begin{array}{c}6484-112 \\
\end{array}$ & $\begin{array}{c}8 \mathrm{D} 5-3 \\
1\end{array}$ & $\begin{array}{l}\text { End-12 } \\
\text { End-12 } \\
\text { End-12 } \\
\text { End-12 } \\
\text { End-12 } \\
\text { End-12 }\end{array}$ & $\begin{array}{l}A C-B C \\
A C-B C \\
A Y-B Y \\
A Y-B Y \\
A E-B E \\
A E-B E\end{array}$ & $\begin{array}{l}\text { Axial } \\
\text { Radial } \\
\text { Axial } \\
\text { Radial } \\
\text { Axial } \\
\text { Radial }\end{array}$ & $\begin{array}{l}14.1 \\
14.1 \\
13.3 \\
13.9 \\
14.3 \\
13.5\end{array}$ & $\begin{array}{l}1.3 \\
1.7 \\
1.0 \\
1.1 \\
1.1 \\
1.9\end{array}$ & $\begin{array}{l}24 \\
20 \\
20 \\
20 \\
20 \\
20\end{array}$ \\
\hline $\begin{array}{l}\text { HLM } \\
\qquad\end{array}$ & $\begin{array}{c}6484-78 \\
\mid\end{array}$ & $\begin{array}{c}367 \\
\mid\end{array}$ & $\begin{array}{l}\text { End-1 } \\
\text { End-1 } \\
\text { End-1 } \\
\text { End-1 } \\
\text { End-1 } \\
\text { End-1 }\end{array}$ & $\begin{array}{l}A C-B C(d) \\
A C-B C \\
A Y-B Y \\
A Y-B Y \\
A E-B E \\
A E-B E\end{array}$ & $\begin{array}{l}\text { Axial } \\
\text { Radial } \\
\text { Axial } \\
\text { Radial } \\
\text { Axial } \\
\text { Radial }\end{array}$ & $\begin{array}{l}16.3 \\
17.9 \\
16.9 \\
17.7 \\
20.8 \\
16.6\end{array}$ & $\begin{array}{l}1.9 \\
1.7 \\
1.8 \\
1.6 \\
1.5 \\
1.4\end{array}$ & $\begin{array}{l}24 \\
20 \\
20 \\
20 \\
20 \\
20\end{array}$ \\
\hline 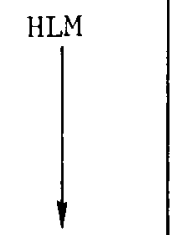 & 6484-78 & $\begin{array}{c}367 \\
\mid\end{array}$ & $\begin{array}{l}\text { Midd 1e-6 } \\
\text { Midd 1e-6 } \\
\text { Midd1e-6 } \\
\text { Midd1e-6 } \\
\text { Midd1e-6 } \\
\text { Midd1e-6 }\end{array}$ & $\begin{array}{l}A C-B C \\
A C-B C \\
A Y-B Y \\
A Y-B Y \\
A E-B E \\
A E-B E\end{array}$ & $\begin{array}{l}\text { Axial } \\
\text { Radial } \\
\text { Axial } \\
\text { Radial } \\
\text { Axial } \\
\text { Radial }\end{array}$ & $\begin{array}{l}13.8 \\
17.5 \\
18.0 \\
17.4 \\
20.8 \\
17.9\end{array}$ & $\begin{array}{l}2.6 \\
2.1 \\
1.7 \\
1.7 \\
1.4 \\
1.3\end{array}$ & $\begin{array}{l}24 \\
20 \\
20 \\
20 \\
20 \\
20\end{array}$ \\
\hline
\end{tabular}

\footnotetext{
(a) $\mathrm{N}$ = number of replicates tested.

(b) See Fig. 5-1 for location in log.

(c) See Figs. 5-4 and 5-1 for location in $10 \mathrm{~g}$.

(d) See Fig. 5-5 for location in $\log$.
} 
TABLE 5-6

ULTIMATE COMPRESSIVE STRENGTH AND MODULUS OF ELASTICITY OF GRADE 2020

\begin{tabular}{|c|c|c|c|c|c|c|c|c|c|}
\hline \multirow{2}{*}{$\begin{array}{c}\text { Log } \\
\text { Number }\end{array}$} & \multirow{2}{*}{$\begin{array}{l}\text { Lot } \\
\text { No. }\end{array}$} & \multirow{2}{*}{$\begin{array}{l}\text { Location } \\
\text { in } \log (a)\end{array}$} & \multirow{2}{*}{$\begin{array}{l}\text { Orientation } \\
\text { of Specimens }\end{array}$} & \multicolumn{3}{|c|}{$\begin{array}{c}\text { Mean Compressive } \\
\text { Strength } \\
(\mathrm{MPa}) \\
\end{array}$} & \multicolumn{3}{|c|}{$\begin{array}{l}\text { Mean Elastic } \\
\text { Modulus } \\
(\mathrm{GPa})\end{array}$} \\
\hline & & & & $\overline{\mathrm{s}}$ & $\sigma$ & $N_{N}^{(b)}$ & $\overline{\mathrm{E}}$ & $\sigma$ & ${ }_{N}(b)$ \\
\hline $\begin{array}{l}6484-110 \\
6410-110\end{array}$ & $\begin{array}{l}728 \\
728\end{array}$ & $\begin{array}{l}\text { Slab } 2 \\
\text { Slab } 2\end{array}$ & $\begin{array}{l}\text { Axial } \\
\text { Radial }\end{array}$ & $\begin{array}{l}78.6 \\
78.5\end{array}$ & $\begin{array}{l}3.1 \\
1.5\end{array}$ & $\begin{array}{l}4 \\
4\end{array}$ & $\begin{array}{r}10.0 \\
9.9\end{array}$ & $\begin{array}{l}0.6 \\
0.9\end{array}$ & $\begin{array}{l}4 \\
4\end{array}$ \\
\hline$\left.\right|^{6484-137}$ & $\begin{array}{l}-- \\
-- \\
-- \\
-- \\
--\end{array}$ & $\begin{array}{ll}\text { Middle slab } & 1 \\
\text { Middle slab } & 1 \\
\text { Middle slab } & 1 \\
\text { Middle slab } & 2 \\
\text { Middle slab } & 2\end{array}$ & $\begin{array}{l}\text { Axial } \\
\text { Radial } \\
\text { Tangential } \\
\text { Radial } \\
\text { Tangential }\end{array}$ & $\begin{array}{l}75.5 \\
74.3 \\
74.2 \\
77.0 \\
79.5\end{array}$ & $\begin{array}{l}1.9 \\
1.5 \\
2.0 \\
1.9 \\
2.0\end{array}$ & $\begin{array}{l}10 \\
10 \\
10 \\
10 \\
10\end{array}$ & $\begin{array}{r}8.7 \\
9.4 \\
9.5 \\
10.1 \\
10.0\end{array}$ & $\begin{array}{l}0.3 \\
0.6 \\
0.5 \\
0.5 \\
0.5\end{array}$ & $\begin{array}{r}9 \\
10 \\
10 \\
10 \\
10\end{array}$ \\
\hline${ }^{6799-00}$ & $\begin{array}{l}-- \\
-- \\
-- \\
-- \\
-- \\
--\end{array}$ & $\begin{array}{l}\text { End slab } 1 \\
\text { End slab } 1 \\
\text { Middle slab } 3 \\
\text { Middle slab } 3 \\
\text { End slab } 5 \\
\text { End slab } 5\end{array}$ & $\begin{array}{l}\text { Axial } \\
\text { Radial } \\
\text { Axial } \\
\text { Radial } \\
\text { Axial } \\
\text { Radial }\end{array}$ & $\begin{array}{l}84.0 \\
82.0 \\
80.0 \\
81.2 \\
85.4 \\
88.9\end{array}$ & $\begin{array}{l}2.5 \\
2.2 \\
1.6 \\
2.7 \\
3.0 \\
4.1\end{array}$ & $\begin{array}{l}8 \\
8 \\
8 \\
8 \\
8 \\
8\end{array}$ & $\begin{array}{l}-- \\
-- \\
-- \\
-- \\
-- \\
--\end{array}$ & $\begin{array}{l}-- \\
-- \\
-- \\
-- \\
-- \\
--\end{array}$ & $\begin{array}{l}-- \\
-- \\
-- \\
-- \\
-- \\
--\end{array}$ \\
\hline
\end{tabular}

(a) See Fig. 5-1 for location in $10 \mathrm{~g}$.

(b) ${ }_{\mathrm{N}}=$ number of replicates tested. 
TABLE 5-7

ULTIMATE COMPRESSIVE STRENGTH AND MODULUS OF ELASTICITY OF GRADE H-440N, LOG 6484-81

\begin{tabular}{|c|c|c|c|c|c|}
\hline \multirow{2}{*}{$\begin{array}{l}\text { Location } \\
\text { in } \log (a)\end{array}$} & \multirow{2}{*}{$\begin{array}{l}\text { Orientation } \\
\text { of Specimens }\end{array}$} & \multicolumn{2}{|c|}{$\begin{array}{c}\text { Mean Compressive } \\
\text { Strength } \\
(\mathrm{MPa})(\mathrm{b})\end{array}$} & \multicolumn{2}{|c|}{$\begin{array}{l}\text { Mean Modulus } \\
\text { of Elasticity } \\
(\mathrm{GPa})(\mathrm{b})\end{array}$} \\
\hline & & $\bar{s}$ & $\sigma$ & $\overline{\mathrm{E}}$ & $\sigma$ \\
\hline End slab 1 & Axial & 52.2 & 2.9 & 5.7 & 0.4 \\
\hline End $s l a b \quad 1$ & Radial & 49.9 & 2.3 & 7.2 & 0.4 \\
\hline Middle slab 6 & Axial & 49.7 & 5.2 & 5.2 & 0.9 \\
\hline Middle slab 6 & Radial & 40.3 & 1.6 & 5.2 & 0.5 \\
\hline
\end{tabular}

(a) See Figs. 5-4 and 5-1(b) for location in $10 \mathrm{~g}$.

(b) Number of replicates tested $=8$. 
TABLE 5-8

ULTIMATE COMPRESSIVE STRENGTH AND MODULUS OF ELASTICITY OF GRADE PGX, LOG 6484-112, LOT 85D-3

\begin{tabular}{|c|c|c|c|c|c|c|}
\hline \multicolumn{2}{|c|}{$\begin{array}{l}\text { Location } \\
\text { in } \log (a)\end{array}$} & \multirow{2}{*}{$\begin{array}{l}\text { Orientation } \\
\text { of Specimens }\end{array}$} & \multicolumn{2}{|c|}{$\begin{array}{c}\text { Mean Compressive } \\
\text { Strength } \\
(\mathrm{MPa})(\mathrm{b})\end{array}$} & \multicolumn{2}{|c|}{$\begin{array}{l}\text { Mean Elastic } \\
\text { Modulus } \\
(\mathrm{GPa})(\mathrm{b})\end{array}$} \\
\hline Slab & Section & & $\overline{\mathrm{S}}$ & $\sigma$ & $\bar{E}$ & $\sigma$ \\
\hline $\begin{array}{c}\text { End- } 1 \\
1\end{array}$ & $\begin{array}{l}A C-B C \\
A C-B C \\
A Y-B Y \\
A Y-B Y \\
A E-B E \\
A E-B E\end{array}$ & $\begin{array}{l}\text { Axial } \\
\text { Radial } \\
\text { Axial } \\
\text { Radial } \\
\text { Axial } \\
\text { Radial }\end{array}$ & $\begin{array}{l}44.7 \\
42.2 \\
43.9 \\
41.1 \\
45.7 \\
42.5\end{array}$ & $\begin{array}{l}0.9 \\
2.7 \\
2.8 \\
2.8 \\
1.6 \\
1.5\end{array}$ & $\begin{array}{l}5.6 \\
5.4 \\
5.2 \\
5.3 \\
5.4 \\
5.4\end{array}$ & $\begin{array}{l}0.2 \\
0.3 \\
0.3 \\
0.5 \\
0.3 \\
0.3\end{array}$ \\
\hline Middle-6 & $\begin{array}{l}A C-B C \\
A C-B C \\
A Y-B Y \\
A Y-B Y \\
A E-B E \\
A E-B E\end{array}$ & $\begin{array}{l}\text { Axial } \\
\text { Radial } \\
\text { Axial } \\
\text { Radial } \\
\text { Axial } \\
\text { Radial }\end{array}$ & $\begin{array}{l}41.7 \\
40.2 \\
44.8 \\
41.8 \\
45.5 \\
43.3\end{array}$ & $\begin{array}{l}2.2 \\
2.2 \\
2.0 \\
1.5 \\
1.9 \\
1.7\end{array}$ & $\begin{array}{l}5.0 \\
6.0 \\
5.9 \\
6.4 \\
5.4 \\
5.5\end{array}$ & $\begin{array}{l}0.3 \\
0.7 \\
0.3 \\
0.1 \\
0.1 \\
0.4\end{array}$ \\
\hline $\begin{array}{c}\text { End- } 12 \\
\mid\end{array}$ & $\begin{array}{l}A C-B C \\
A C-B C \\
A Y-B Y \\
A Y-B Y \\
A E-B E \\
A E-B E\end{array}$ & $\begin{array}{l}\text { Axial } \\
\text { Radial } \\
\text { Axial } \\
\text { Radial } \\
\text { Axial } \\
\text { Radial }\end{array}$ & $\begin{array}{l}41.7 \\
40.2 \\
40.3 \\
39.1 \\
40.7 \\
38.3\end{array}$ & $\begin{array}{l}0.8 \\
1.4 \\
1.9 \\
1.1 \\
2.1 \\
1.8\end{array}$ & $\begin{array}{l}4.9 \\
5.8 \\
4.5 \\
5.5 \\
4.7 \\
5.3\end{array}$ & $\begin{array}{l}0.2 \\
0.4 \\
0.2 \\
0.3 \\
0.2 \\
0.1\end{array}$ \\
\hline
\end{tabular}
(a) See Fig. 5-5 for location in 10 .
(b) Number of rep1icates tested $=8$. 
TABLE 5-9

ULTIMATE COMPRESSIVE STRENGTH AND MODULUS OF ELASTICITY OF GRADE HLM, LOG 6484-78, LOT 367

\begin{tabular}{|c|c|c|c|c|c|c|}
\hline \multicolumn{2}{|c|}{$\begin{array}{l}\text { Location } \\
\text { in } \log (a)\end{array}$} & \multirow{2}{*}{$\begin{array}{l}\text { Orientation } \\
\text { of Specimens }\end{array}$} & \multicolumn{2}{|c|}{$\begin{array}{c}\text { Mean Compressive } \\
\text { Strength } \\
(\mathrm{MPa})(\mathrm{b})\end{array}$} & \multicolumn{2}{|c|}{$\begin{array}{l}\text { Mean Elastic } \\
\text { Modulus } \\
(\text { GPa) (b) }\end{array}$} \\
\hline Slab & Section & & $\bar{S}$ & $\sigma$ & $\overline{\mathrm{E}}$ & $\sigma$ \\
\hline $\begin{array}{l}\text { End }-1 \\
\mid\end{array}$ & $\begin{array}{l}A C-B C \\
A C-B C \\
A Y-B Y \\
A Y-B Y \\
A E-B E \\
A E-B E\end{array}$ & $\begin{array}{l}\text { Axial } \\
\text { Radial } \\
\text { Axial } \\
\text { Radial } \\
\text { Axial } \\
\text { Radial }\end{array}$ & $\begin{array}{l}38.1 \\
43.0 \\
35.0 \\
41.0 \\
39.8 \\
41.6\end{array}$ & $\begin{array}{l}0.9 \\
1.1 \\
1.5 \\
1.8 \\
1.5 \\
2.4\end{array}$ & $\begin{array}{l}5.1 \\
5.9 \\
5.0 \\
5.7 \\
6.7 \\
5.3\end{array}$ & $\begin{array}{l}0.4 \\
0.3 \\
0.6 \\
0.4 \\
0.3 \\
0.3\end{array}$ \\
\hline Midd $1 e-6$ & $\begin{array}{l}A C-B C \\
A C-B C \\
A Y-B Y \\
A Y-B Y \\
A E-B E \\
A E-B E\end{array}$ & $\begin{array}{l}\text { Axial } \\
\text { Radial } \\
\text { Axial } \\
\text { Radial } \\
\text { Axial } \\
\text { Radial }\end{array}$ & $\begin{array}{l}35.3 \\
40.5 \\
45.4 \\
42.6 \\
41.1 \\
44.7\end{array}$ & $\begin{array}{l}2.2 \\
1.6 \\
3.1 \\
1.5 \\
1.8 \\
1.0\end{array}$ & $\begin{array}{l}5.3 \\
5.9 \\
5.7 \\
5.8 \\
7.1 \\
5.9\end{array}$ & $\begin{array}{l}0.6 \\
0.4 \\
0.8 \\
0.4 \\
0.7 \\
0.2\end{array}$ \\
\hline
\end{tabular}

(a) See Fig. 5-5 for location in $\log$.

(b) Number of replicates tested $=8$. 
TABLE 5-10

DISTRIBUTION OF IMPURITY CONTENT WITHIN GRADE 2020, LOG 6484-110, LOT 728

\begin{tabular}{|c|c|c|c|c|c|c|c|c|c|c|c|c|c|c|c|c|c|c|c|c|c|}
\hline \multirow{3}{*}{$\begin{array}{l}\text { Sample } \\
\text { Number }\end{array}$} & \multicolumn{2}{|c|}{ Location in Log $(a)$} & \multirow{2}{*}{\multicolumn{19}{|c|}{ Element (ppm) }} \\
\hline & \multirow[b]{2}{*}{ Slab } & \multirow{2}{*}{$\begin{array}{c}\text { Radial } \\
\text { Location }\end{array}$} & & & & & & & & & & & & & & & & & & & \\
\hline & & & Ash & $\mathrm{S}$ & $\mathrm{A} 1$ & B & $\mathrm{Ba}$ & $\mathrm{Ca}$ & $\mathrm{Cu}$ & $\mathrm{Fe}$ & $\mathrm{K}$ & Li & $\mathrm{Mg}$ & Mn & $\mathrm{Na}$ & $\mathrm{Ni}$ & $\mathrm{Pb}$ & Si & $\mathrm{Sr}$ & $\mathrm{Ti}$ & V \\
\hline L16B & End-1 & Edge & 2000 & 23 & 60 & 4 & 40 & 600 & $<1$ & 400 & 80 & $<1$ & 4 & 2 & 10 & $<4$ & $<6$ & 200 & 40 & 40 & 20 \\
\hline L28B & End-1 & Edge & 1900 & 13 & 60 & 2 & 40 & 600 & $<1$ & 400 & 60 & $<1$ & 2 & 1 & 10 & $<4$ & $<6$ & 200 & $<40$ & 20 & 10 \\
\hline L64B & Midd $1 e-6$ & Edge & 1100 & 11 & 20 & 4 & 40 & 400 & $<1$ & 20 & 20 & $<1$ & 4 & $<1$ & 10 & $<4$ & $<6$ & 20 & $<40$ & 100 & 20 \\
\hline L76B & Middle-6 & Edge & 1200 & 12 & 60 & 4 & 20 & 400 & $<1$ & 60 & 60 & 8 & 4 & $<1$ & 20 & $<4$ & $<6$ & 100 & $<40$ & 100 & 20 \\
\hline
\end{tabular}

(a) See Fig. 5-1 for location in log. 
TABIE 5-11

DISTRIBUTION OF IMPURITY CONTENT WITHIN GRADE H-440N, LOG 6484-81

\begin{tabular}{|c|c|c|c|c|c|c|c|c|c|c|c|c|c|c|c|c|c|c|c|c|c|c|}
\hline \multirow{2}{*}{$\begin{array}{l}\text { Sample } \\
\text { Number }\end{array}$} & \multicolumn{2}{|c|}{$\begin{array}{l}\text { Location } \\
\text { in Log (a) }\end{array}$} & \multirow{2}{*}{$\begin{array}{c}\text { Circumfer- } \\
\text { ential } \\
\text { Location } \\
\text { (rad) } \\
\end{array}$} & \multicolumn{19}{|c|}{ Element (ppm) } \\
\hline & Slab & Radia1 & & Ash & $\mathrm{S}$ & $\mathrm{AI}$ & B & $\mathrm{Ba}$ & $\mathrm{Ca}$ & $\mathrm{Cu}$ & $\mathrm{Fe}$ & $\mathrm{K}$ & $\mathrm{Li}$ & $\mathrm{Mg}$ & $\mathrm{Mn}$ & $\mathrm{Na}$ & $\mathrm{Ni}$ & $\mathrm{Pb}$ & $\mathrm{Si}$ & $\mathrm{Sr}$ & $\mathrm{Ti}$ & $\mathrm{V}$ \\
\hline $\mathrm{L} 2$ & (b) & Edge & 0 & 590 & 104 & $<1$ & 2 & 20 & 60 & $<1$ & 10 & 10 & $<0.5$ & 4 & $<1$ & $<40$ & $<4$ & $<6$ & 80 & $<40$ & 40 & 60 \\
\hline L3 & (b) & Edge & 0.78 & 680 & 114 & $<1$ & 2 & 20 & 60 & $<1$ & 8 & 6 & $<0.5$ & 1 & $<1$ & $<40$ & $<4$ & $<6$ & 80 & $<40$ & 20 & 40 \\
\hline L21 & (b) & Edge & 2.36 & 680 & 9 & $<1$ & 2 & 20 & 60 & $<1$ & 10 & 8 & $<0.5$ & 10 & $<1$ & $<40$ & $<4$ & $<6$ & 80 & $<40$ & 20 & 20 \\
\hline L16 & (b) & Edge & 3.14 & 660 & 5 & $<1$ & 2 & 20 & 60 & $<1$ & 10 & 6 & $<0.5$ & 10 & $<1$ & $<40$ & $<4$ & $<6$ & 80 & $<40$ & 20 & 20 \\
\hline $\mathrm{L} 40$ & (b) & Edge & 3.14 & 620 & 11 & $<1$ & 2 & 20 & 60 & $<1$ & 20 & 8 & $<0.5$ & 10 & $<1$ & $<40$ & $<4$ & $<6$ & 80 & $<40$ & 40 & 20 \\
\hline L39 & (b) & Edge & 3.92 & 620 & $<2$ & $<1$ & 2 & 8 & 60 & $<1$ & $<20$ & 10 & $<0.5$ & 10 & $<1$ & $<40$ & $<4$ & $<6$ & 80 & $<40$ & 20 & 40 \\
\hline $\mathrm{L} 57$ & (b) & Edge & 5.49 & 550 & $<2$ & $<1$ & 2 & 10 & 60 & $<1$ & 10 & 6 & $<0.5$ & 2 & $<1$ & $<40$ & $<4$ & $<6$ & 80 & $<40$ & 20 & 20 \\
\hline L 10 & (b) & Center & -- & 700 & 20 & $<1$ & 2 & 20 & 60 & $<1$ & 10 & 6 & $<0.5$ & 10 & $<1$ & $<40$ & $<4$ & $<6$ & 80 & $<40$ & 20 & 40 \\
\hline L52 & (c) & Edge & 0 & 520 & 4 & $<1$ & 2 & 20 & 60 & $<1$ & 10 & 6 & $<0.5$ & 20 & $<1$ & $<40$ & $<4$ & $<6$ & 80 & $<40$ & 20 & 10 \\
\hline L76 & (c) & Edge & 0 & 520 & $<2$ & $<1$ & 2 & 10 & 60 & $<1$ & 20 & 4 & $<0.5$ & 10 & $<1$ & $<40$ & $<4$ & $<6$ & 80 & $<40$ & 40 & 40 \\
\hline L75 & (c) & Edge & 0.78 & 590 & $<3$ & $<1$ & 2 & 10 & 60 & $<1$ & 10 & 4 & $<0.5$ & 20 & $<1$ & $<40$ & $<4$ & $<6$ & 80 & $<40$ & 20 & 40 \\
\hline L93 & (c) & Edge & 2.36 & 610 & 12 & $<1$ & 2 & 10 & 60 & $<1$ & 10 & 4 & $<0.5$ & 10 & $<1$ & $<40$ & $<4$ & $<6$ & 80 & $<40$ & 40 & 40 \\
\hline L64 & (c) & Edge & 3.14 & 500 & $<4$ & $<1$ & 2 & 10 & 60 & $<1$ & 10 & 8 & $<0.5$ & 20 & $<1$ & $<40$ & $<4$ & $<6$ & 80 & $<40$ & 20 & 10 \\
\hline L88 & (c) & Edge & 3.14 & 580 & 11 & $<1$ & 2 & 10 & 60 & $<1$ & 20 & 8 & $<0.5$ & 10 & $<1$ & $<40$ & $<4$ & $<6$ & 80 & $<40$ & 40 & 40 \\
\hline L 111 & (c) & Edge & 3.92 & 570 & $<2$ & $<1$ & 2 & 10 & 60 & $<1$ & 20 & 8 & $<0.5$ & 10 & $<1$ & $<40$ & $<4$ & $<6$ & 80 & $<40$ & 40 & 40 \\
\hline L 129 & (c) & Edge & 5.49 & 540 & 13 & $<1$ & 2 & 10 & 40 & $<1$ & 20 & 8 & $<0.5$ & 20 & $<1$ & $<40$ & $<4$ & $<6$ & 80 & $<40$ & 40 & 40 \\
\hline L82 & (c) & Center & -- & 630 & 14 & $<1$ & 2 & 10 & 60 & $<1$ & 10 & $<4$ & $<0.5$ & 10 & $<1$ & $<40$ & $<4$ & $<6$ & 80 & $<40$ & 10 & 20 \\
\hline L58 & (c) & Center & -- & 570 & 12 & $<1$ & 2 & 10 & 60 & $<1$ & 20 & 6 & $<0.5$ & 10 & $<1$ & $<40$ & $<4$ & $<6$ & 80 & $<40$ & 20 & 20 \\
\hline
\end{tabular}

(a) See Figs. 5-4 and 5-1(b) for location in $10 \mathrm{~g}$.

(b) End-1.

(c) Middle-6. 
TABLE $\quad 5-12$

DISTRIBUTION OF IMPURITY CONTENT WITHIN GRADE PGX, LOG 6484-112, LOT 8D5-3

\begin{tabular}{|c|c|c|c|c|c|c|c|c|c|c|c|c|c|c|c|c|c|c|c|c|c|}
\hline \multirow{3}{*}{$\begin{array}{l}\text { Sample } \\
\text { Number }\end{array}$} & \multicolumn{2}{|c|}{ Location in $\log (a)$} & \multirow{2}{*}{\multicolumn{19}{|c|}{ Element (ppm) }} \\
\hline & \multirow[b]{2}{*}{ Slab } & \multirow{2}{*}{$\begin{array}{c}\text { Radial } \\
\text { Location }\end{array}$} & & & & & & & & & & & & & & & & & & & \\
\hline & & & Ash & $\mathrm{S}$ & $\mathrm{Al}$ & B & $\mathrm{Ba}$ & $\mathrm{Ca}$ & $\mathrm{Cu}$ & $\mathrm{Fe}$ & $\mathrm{K}$ & $\mathrm{Li}$ & $\mathrm{Mg}$ & Mn & $\mathrm{Na}$ & $\mathrm{Ni}$ & $\mathrm{Pb}$ & $\mathrm{Si}$ & $\mathrm{Sr}$ & $\mathrm{Ti}$ & $\mathrm{V}$ \\
\hline E70B & Top- 1 & $\mathrm{E}-\mathrm{A}$ & 6740 & 110 & 80 & 2 & 40 & 80 & 10 & 1000 & 10 & 1 & 1 & 10 & $<10$ & 20 & $<6$ & 100 & $<40$ & 40 & 10 \\
\hline $\mathrm{E} 40 \mathrm{~B}$ & Top-1 & $\mathrm{EC}-\mathrm{A}$ & 6890 & 355 & 80 & 2 & 40 & 80 & 10 & 1000 & 60 & 6 & 10 & 10 & 10 & 20 & $<6$ & 40 & $<40$ & 20 & 10 \\
\hline E16B & Top-1 & $\mathrm{C}-\mathrm{A}$ & 7600 & 540 & 100 & 2 & 40 & 100 & 10 & $>1000$ & 20 & 2 & 40 & 40 & $<10$ & 40 & $<6$ & 40 & $<40$ & 40 & 20 \\
\hline $\mathrm{E} 88 \mathrm{~B}$ & Top-1 & $C-B$ & 6800 & 570 & 80 & 2 & 40 & 80 & 10 & 1000 & 20 & 6 & 40 & 20 & 20 & 20 & $<6$ & 20 & $<40$ & 40 & 20 \\
\hline E124B & Top-1 & $\mathrm{EC}-\mathrm{B}$ & 7440 & 430 & 80 & 2 & 40 & 80 & 20 & 1000 & 20 & 2 & 10 & 20 & $<10$ & 20 & $<6$ & 80 & $<40$ & 40 & 20 \\
\hline E154B & Top-1 & $E-B$ & 7160 & 1005 & 100 & 2 & 60 & $>1000$ & 8 & 600 & $<10$ & $<1$ & 6 & 1 & $<10$ & $<4$ & $<6$ & 80 & $<40$ & 40 & 20 \\
\hline M7OB & Middle-6 & $\mathrm{E}-\mathrm{A}$ & 5310 & 150 & 80 & 2 & 40 & 8 & 10 & 1000 & 80 & 2 & 8 & 10 & 20 & 20 & $<6$ & 60 & $<40$ & 80 & 40 \\
\hline $\mathrm{M} 40 \mathrm{~B}$ & Middle- 6 & $\mathrm{EC}-\mathrm{A}$ & 8070 & 310 & 80 & 2 & 40 & 100 & 10 & 1000 & 20 & 2 & 2 & 10 & 10 & 20 & $<6$ & 80 & $<40$ & 40 & 20 \\
\hline M16B & Middle- 6 & $\mathrm{C}-\mathrm{A}$ & 7010 & 325 & 100 & 2 & 40 & 80 & 10 & $>1000$ & 20 & 1 & 10 & 40 & $<10$ & 20 & $<6$ & 40 & $<40$ & 40 & 20 \\
\hline M100B & Middle-6 & $C-B$ & 2779 & 430 & 80 & 2 & 40 & 200 & 20 & $>1000$ & $<10$ & 2 & 20 & 40 & $<10$ & 20 & $<6$ & 40 & $<40$ & 40 & 20 \\
\hline M1 22B & Middle- 6 & $\mathrm{EC}-\mathrm{B}$ & 893 & 455 & 100 & 2 & 40 & $>1000$ & 10 & $>1000$ & 10 & 2 & 1 & 40 & $<10$ & 20 & $<6$ & 100 & $<40$ & 40 & 20 \\
\hline M152B & Middle- 6 & $E-B$ & 880 & 125 & 80 & $<0.5$ & 40 & 60 & 6 & 40 & 10 & $<1$ & $<0.5$ & $<1$ & $<10$ & $<4$ & $<6$ & 40 & $<40$ & 40 & 4 \\
\hline B 70B & Bottom- 12 & $\mathrm{E}-\mathrm{A}$ & 11070 & 720 & 200 & 2 & 60 & $>1000$ & 20 & $>1000$ & 20 & 1 & 4 & 40 & $<10$ & 20 & $<6$ & 400 & $<40$ & 40 & 40 \\
\hline $\mathrm{B} 40 \mathrm{~B}$ & Bottom- 12 & $\mathrm{EC}-\mathrm{A}$ & 10440 & 610 & 60 & 2 & 40 & 200 & 20 & $>1000$ & 10 & 2 & 10 & 40 & $<10$ & 20 & $<6$ & 80 & $<40$ & 40 & 20 \\
\hline $\mathrm{B} 4 \mathrm{~B}$ & Bottom- 12 & $C-A$ & 7150 & 875 & 60 & 2 & 40 & 200 & 20 & $>1000$ & 10 & 2 & 40 & 40 & $<10$ & 20 & $<6$ & 20 & $<40$ & 40 & 20 \\
\hline $\mathrm{B} 88 \mathrm{~B}$ & Bot tom-12 & $C-B$ & 7270 & 1096 & 100 & 2 & 40 & 100 & 20 & $>1000$ & 40 & 2 & 60 & 60 & 10 & 20 & $<6$ & 40 & $<40$ & 40 & 20 \\
\hline B124B & Bottom-12 & $\mathrm{EC}-\mathrm{B}$ & 845 & 695 & 80 & 2 & 40 & 80 & 20 & $>1000$ & 40 & 1 & 20 & 40 & 10 & 20 & $<6$ & 60 & $<40$ & 40 & 20 \\
\hline B154B & Bottom- 12 & $E-B$ & 6990 & 945 & 80 & 2 & 40 & $>1000$ & 20 & $>1000$ & 60 & 1 & 10 & 40 & $<10$ & 20 & $<6$ & 60 & $<40$ & 40 & 20 \\
\hline
\end{tabular}

(a) See Fig. 5-5 for location in log. 
TABLE 5-13

DISTRIBUTION OF IMPURITY CONTENT WITHIN GRADE HLM, LOG 6484-78, LOT 367

\begin{tabular}{|c|c|c|c|c|c|c|c|c|c|c|c|c|c|c|c|c|c|c|c|c|c|}
\hline \multirow{3}{*}{$\begin{array}{l}\text { Sample } \\
\text { Number }\end{array}$} & \multicolumn{2}{|c|}{ Location in Log $(a)$} & \multirow{2}{*}{\multicolumn{19}{|c|}{ Element (ppm) }} \\
\hline & \multirow[b]{2}{*}{ Slab } & \multirow{2}{*}{$\begin{array}{l}\text { Radial } \\
\text { Location }\end{array}$} & & & & & & & & & & & & & & & & & & & \\
\hline & & & Ash & $\mathrm{s}$ & $\mathrm{A} 1$ & B & $\mathrm{Ba}$ & $\mathrm{Ca}$ & $\mathrm{Cu}$ & $\mathrm{Fe}$ & $\mathrm{K}$ & $\mathrm{Li}$ & $\mathrm{Mg}$ & Mn & $\mathrm{Na}$ & $\mathrm{Ni}$ & $\mathrm{Pb}$ & $\mathrm{Si}$ & $\mathrm{Sr}$ & Ti & $\mathrm{V}$ \\
\hline E70B & Top-1 & $E-A$ & 2400 & 176 & 60 & 2 & 60 & 400 & $<1$ & 1000 & 20 & $<1$ & 2 & $<1$ & 10 & 20 & $<6$ & 100 & $<40$ & 60 & 60 \\
\hline E4OB & Top-1 & $E C-A$ & 4900 & $<4$ & 60 & 2 & 40 & 200 & $<1$ & $>1000$ & 10 & 1 & 6 & 4 & 10 & 40 & $<6$ & 80 & $<40$ & 40 & 40 \\
\hline E16B & Top-1 & $\mathrm{C}-\mathrm{A}$ & 4500 & $<4$ & 80 & 20 & 40 & 200 & $<1$ & $>1000$ & 10 & $<1$ & 2 & 4 & 10 & 40 & $<6$ & 60 & $<40$ & 40 & 40 \\
\hline E88B & Top-1 & $\mathrm{C}-\mathrm{B}$ & 4600 & 58 & 40 & 2 & 40 & 200 & $<1$ & $>1000$ & 60 & 2 & 4 & 4 & 40 & 40 & $<6$ & 60 & $<40$ & 40 & 40 \\
\hline E124B & Top-1 & EC-B & 4500 & 18 & 20 & 2 & 40 & 200 & $<1$ & $>1000$ & $<10$ & 1 & 2 & 4 & $<10$ & 40 & $<6$ & 60 & $<40$ & 40 & 60 \\
\hline E154B & Top- 1 & $E-B$ & 1200 & 16 & $<1$ & 2 & 40 & 100 & $<1$ & 800 & $<10$ & $<1$ & 4 & $<1$ & $<10$ & 10 & $<6$ & 80 & $<40$ & 40 & 20 \\
\hline M70B & Midd le-6 & $E-A$ & 670 & 45 & $<1$ & 2 & 40 & 80 & $<1$ & 100 & 10 & 2 & 2 & $<1$ & $<10$ & $<4$ & $<6$ & 60 & $<40$ & 20 & 10 \\
\hline $\mathrm{M} 40 \mathrm{~B}$ & Middle- 6 & EC-A & 1900 & $<4$ & 60 & 2 & 40 & 200 & $<1$ & 800 & $<10$ & 1 & 4 & 2 & $<10$ & 20 & $<6$ & 100 & $<40$ & 40 & 40 \\
\hline M16B & Middle- 6 & $\mathrm{C}-\mathrm{A}$ & 1000 & $<4$ & 80 & 2 & 40 & 100 & $<1$ & $>1000$ & $<10$ & 6 & 4 & 100 & $<10$ & $>1000$ & $<6$ & 200 & $<40$ & 80 & 20 \\
\hline M100B & Middle-6 & $C-B$ & 1100 & 23 & 40 & 2 & 20 & 100 & $<1$ & 100 & $<10$ & 1 & 4 & 20 & $<10$ & 200 & $<6$ & 80 & $<40$ & 20 & 20 \\
\hline M122B & Middle-6 & $E C-B$ & 1100 & 22 & 100 & 2 & 40 & 100 & $<1$ & 400 & 10 & 1 & 2 & $<1$ & $<10$ & 10 & $<6$ & 80 & $<40$ & 20 & 20 \\
\hline M152B & Middle- 6 & $E-B$ & 900 & 9 & $<1$ & 2 & 40 & 80 & $<1$ & 80 & $<10$ & $<1$ & 2 & $<1$ & $<10$ & 10 & $<6$ & 80 & $<40$ & 20 & 20 \\
\hline
\end{tabular}

${ }^{(a)}$ See Fig. 5-5 for location in $10 \mathrm{~g}$. 


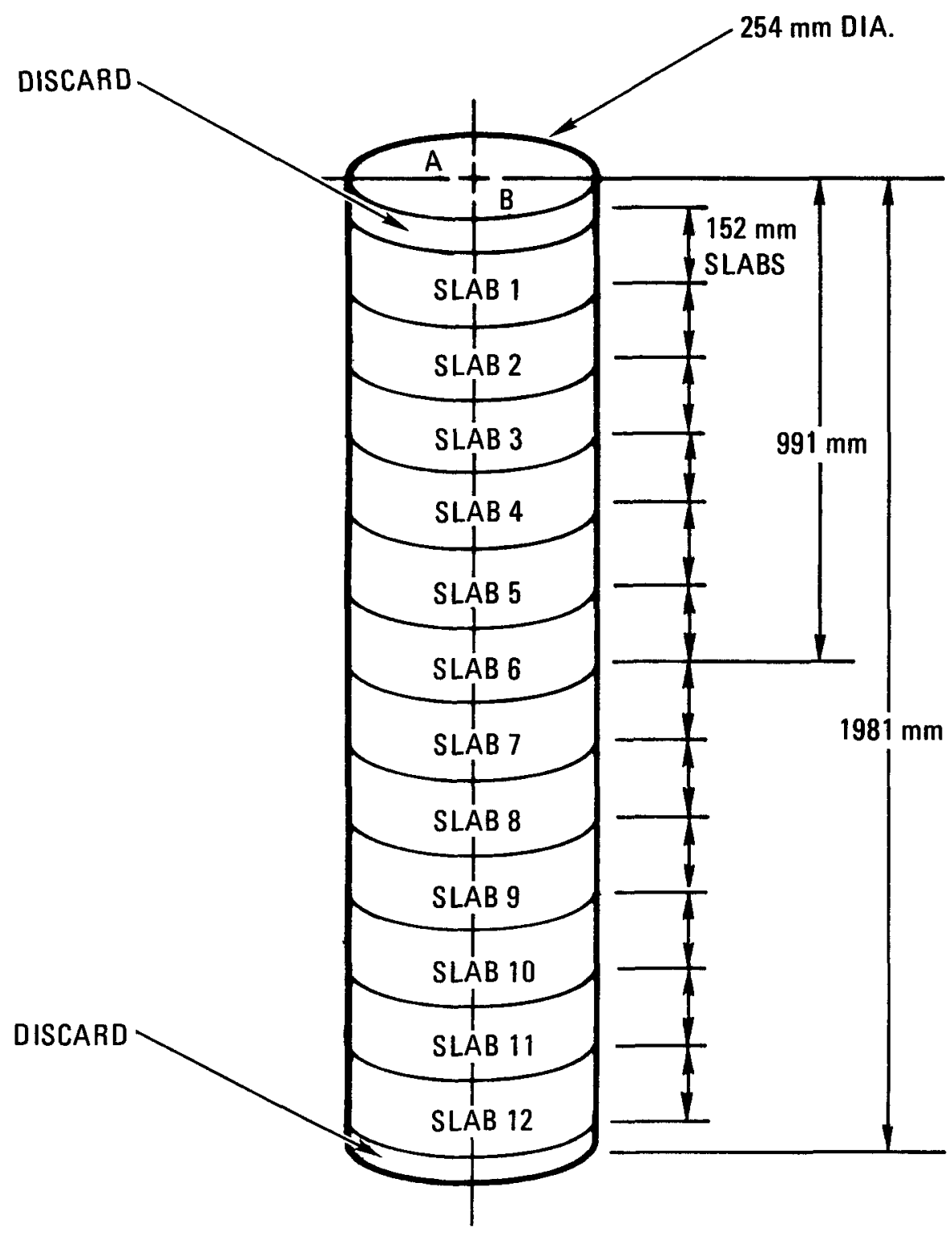

Fig. 5-1(a). Slab diagram for core support post graphite grade 2020, $\log 6484-110$ 


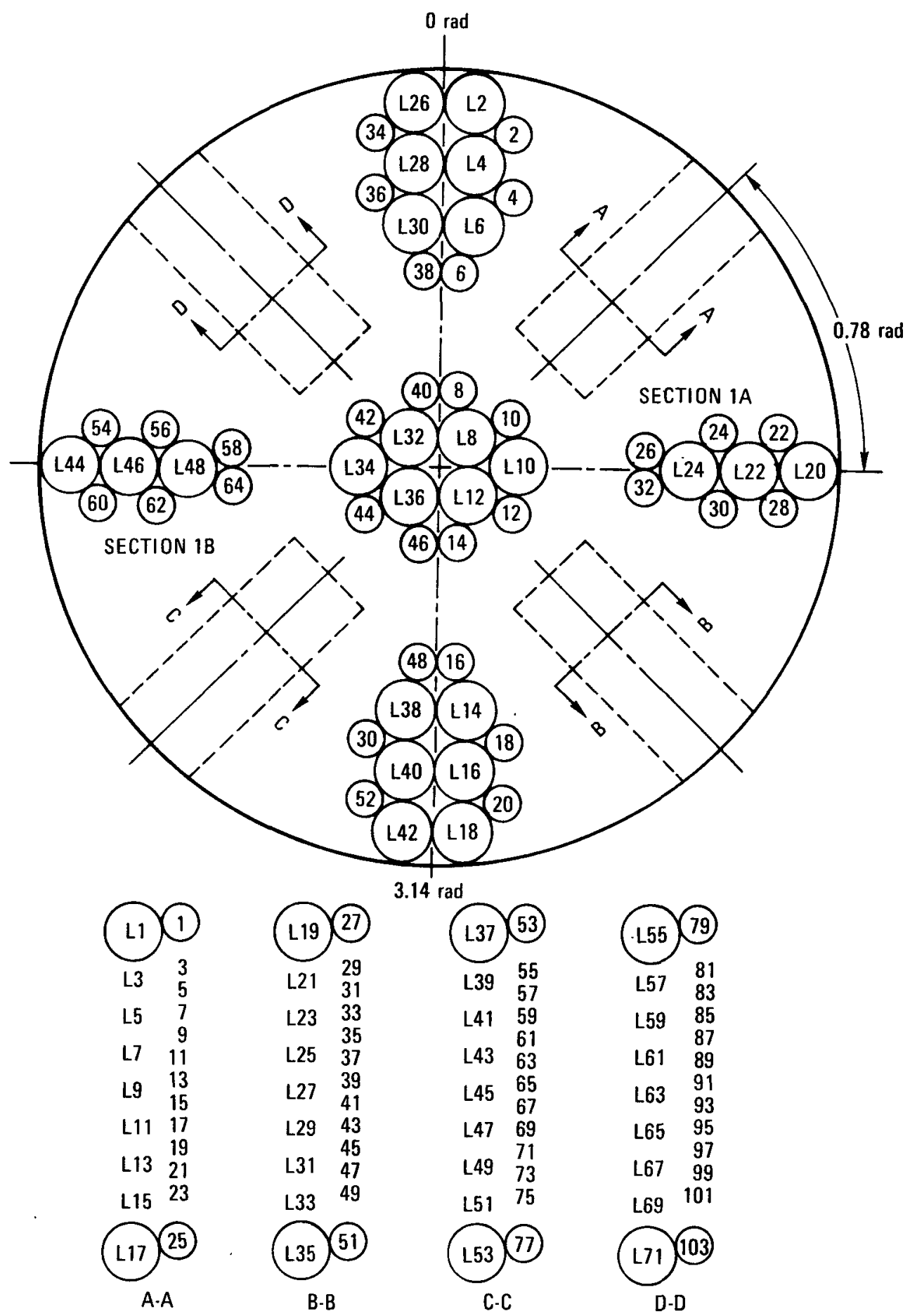

Fig. 5-1(b). Core sampling plan of a slab of grade 2020, log 6484-110 

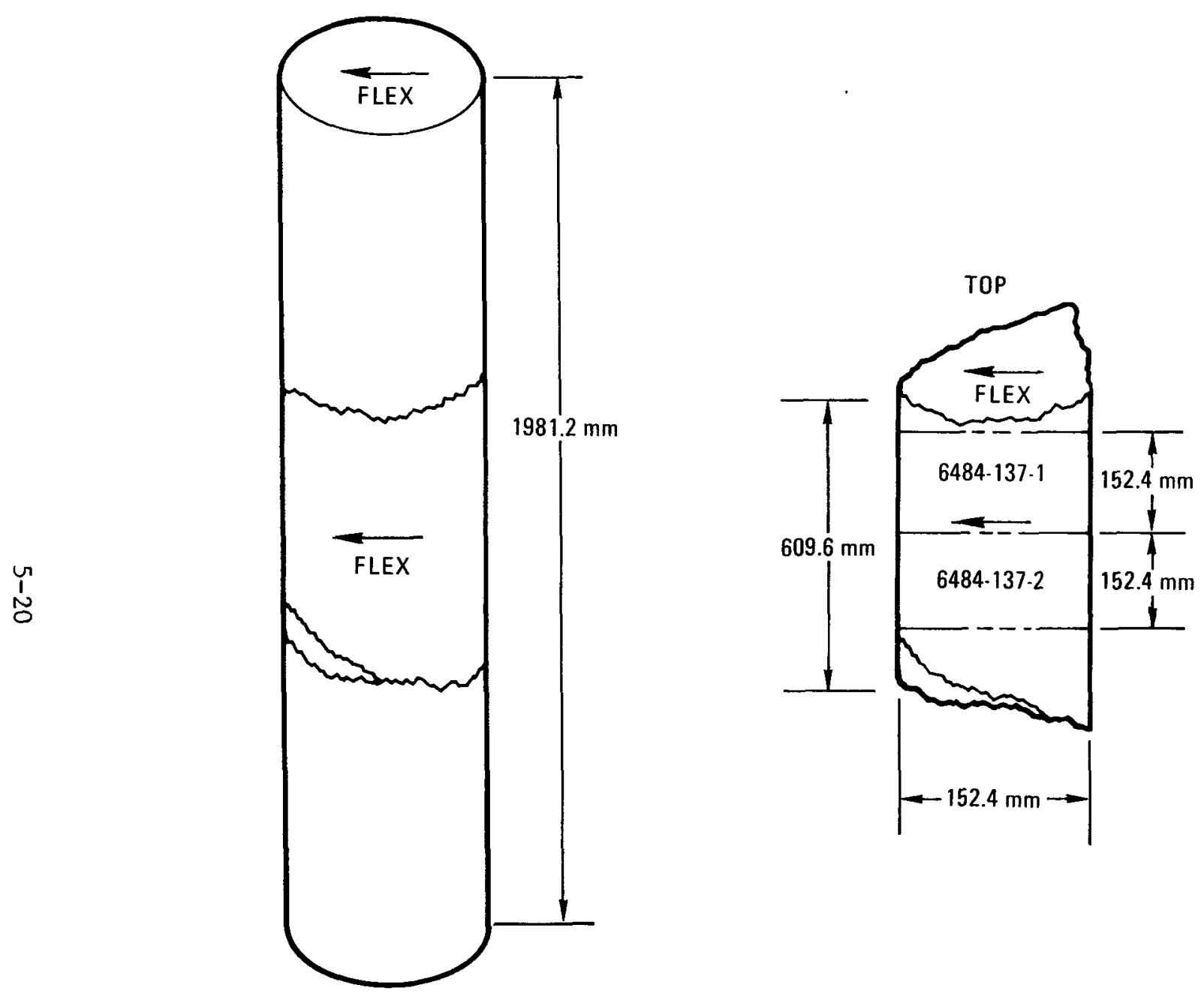

SAMPLE

$12.7 \mathrm{~mm}$

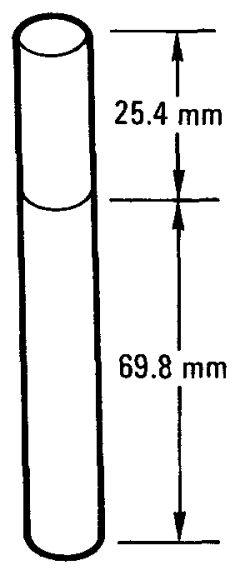

Fig. 5-2(a). Slab diagram for core support post graphite grade 2020 (fracture piece taken for test, $\log 6484-137$ ) 


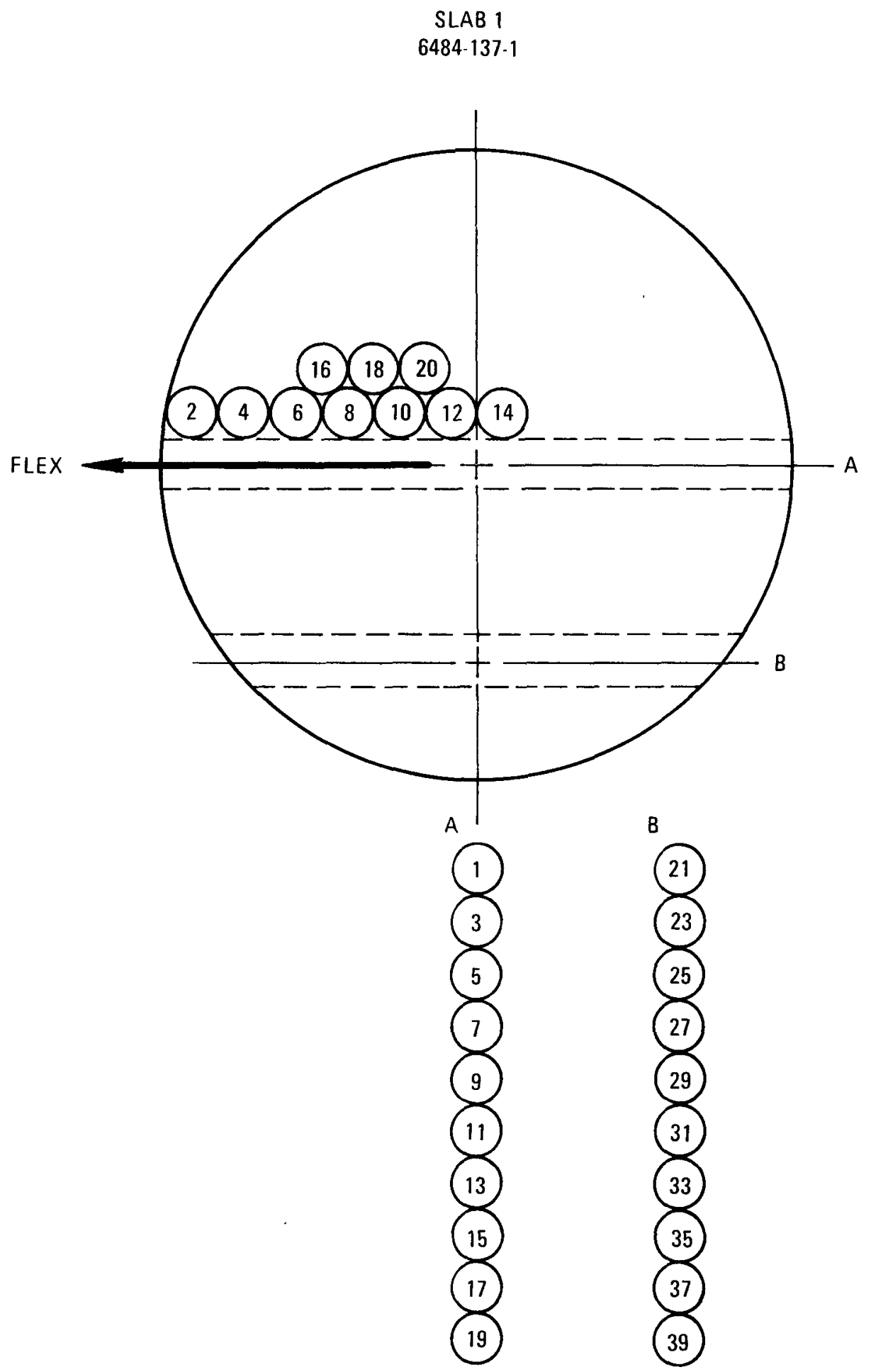

Fig. 5-2(b). Core sampling and specimen orientation of grade 2020, log 6484-137; axia1, radial, and tangential specimens parallel to flex 


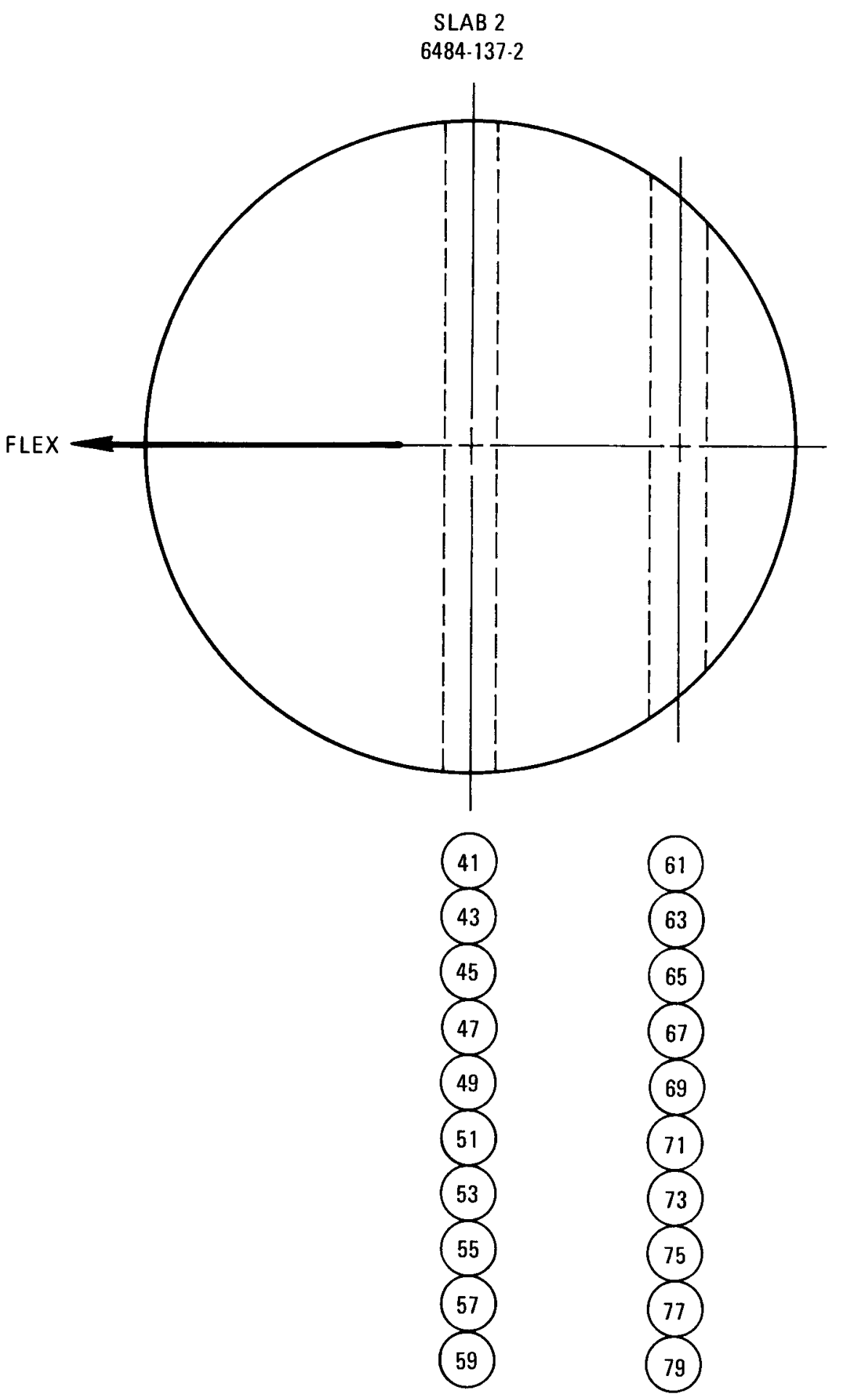

Fig. 5-2(c). Core sampling and specimen orientation of grade $2020,10 \mathrm{~g}$ 6484-137; radial and tangential specimens perpendicular to flex 


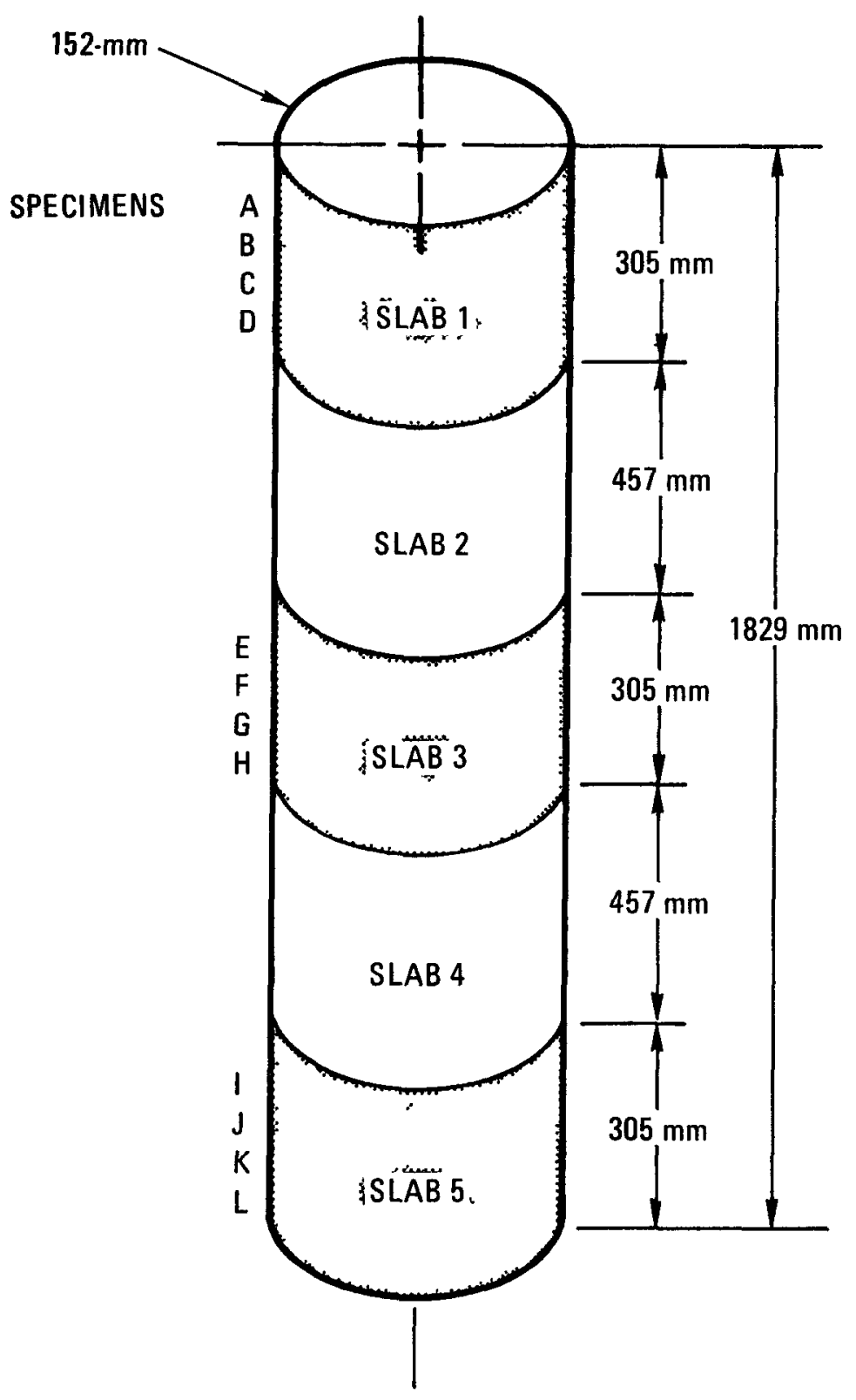

Fig. 5-3(a). Slab diagram for core support post graphite grade 2020, log 6799-00 


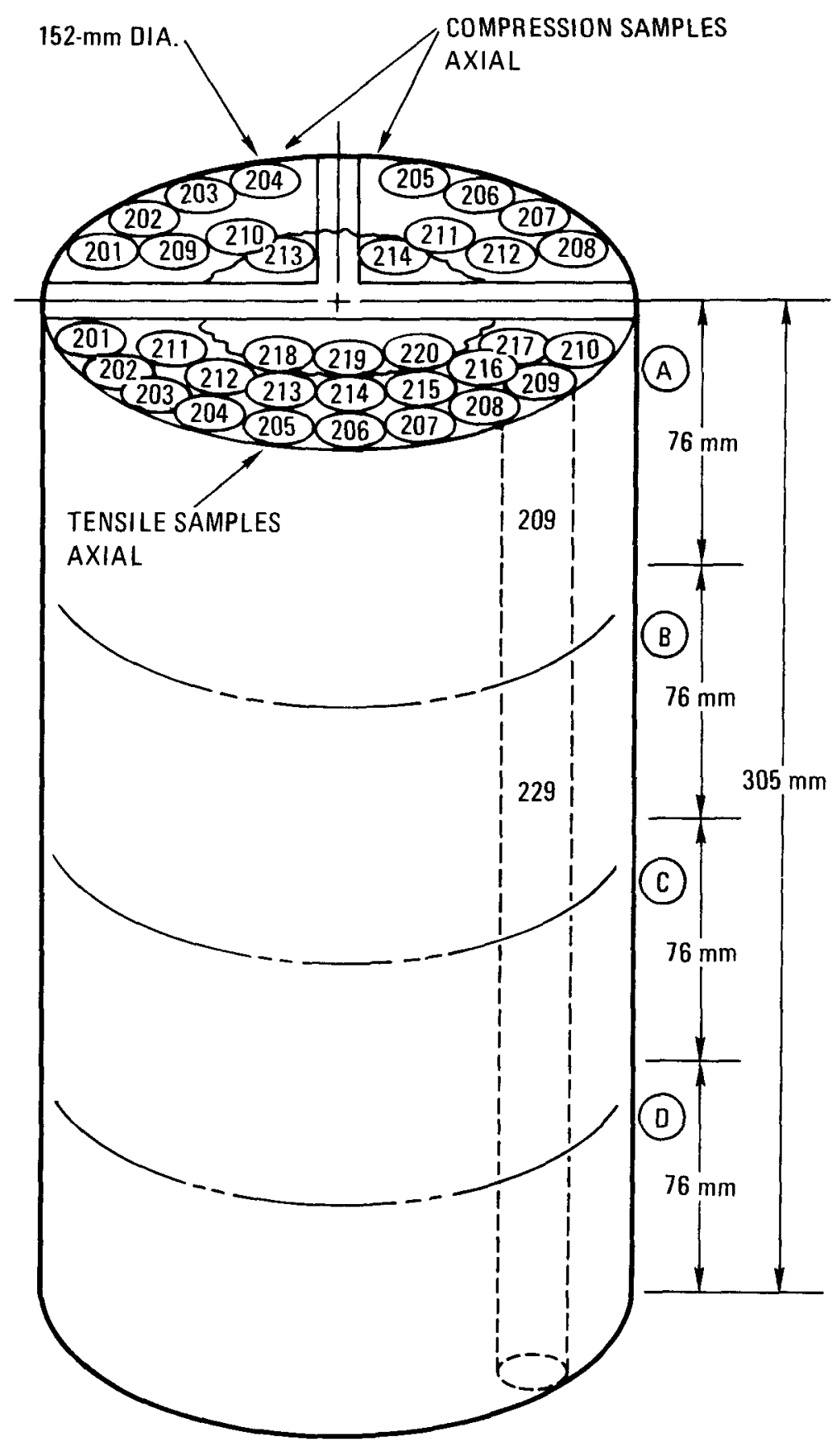

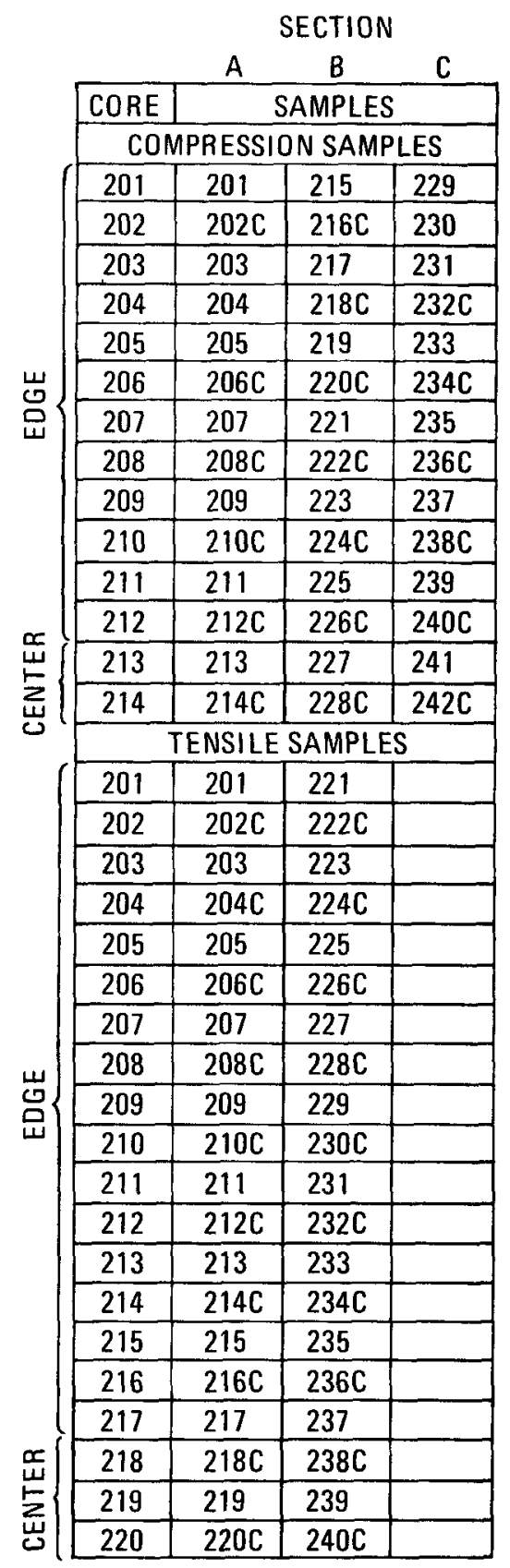

FINISHED CORES 12.8-mm DIA.

Fig. 5-3(b). Core sampling plan of slab 1 of grade 2020, $\log 6799-00$; axial (core sampling of slabs 3 and 5 was the same as for $\mathrm{slab}$ 1) 


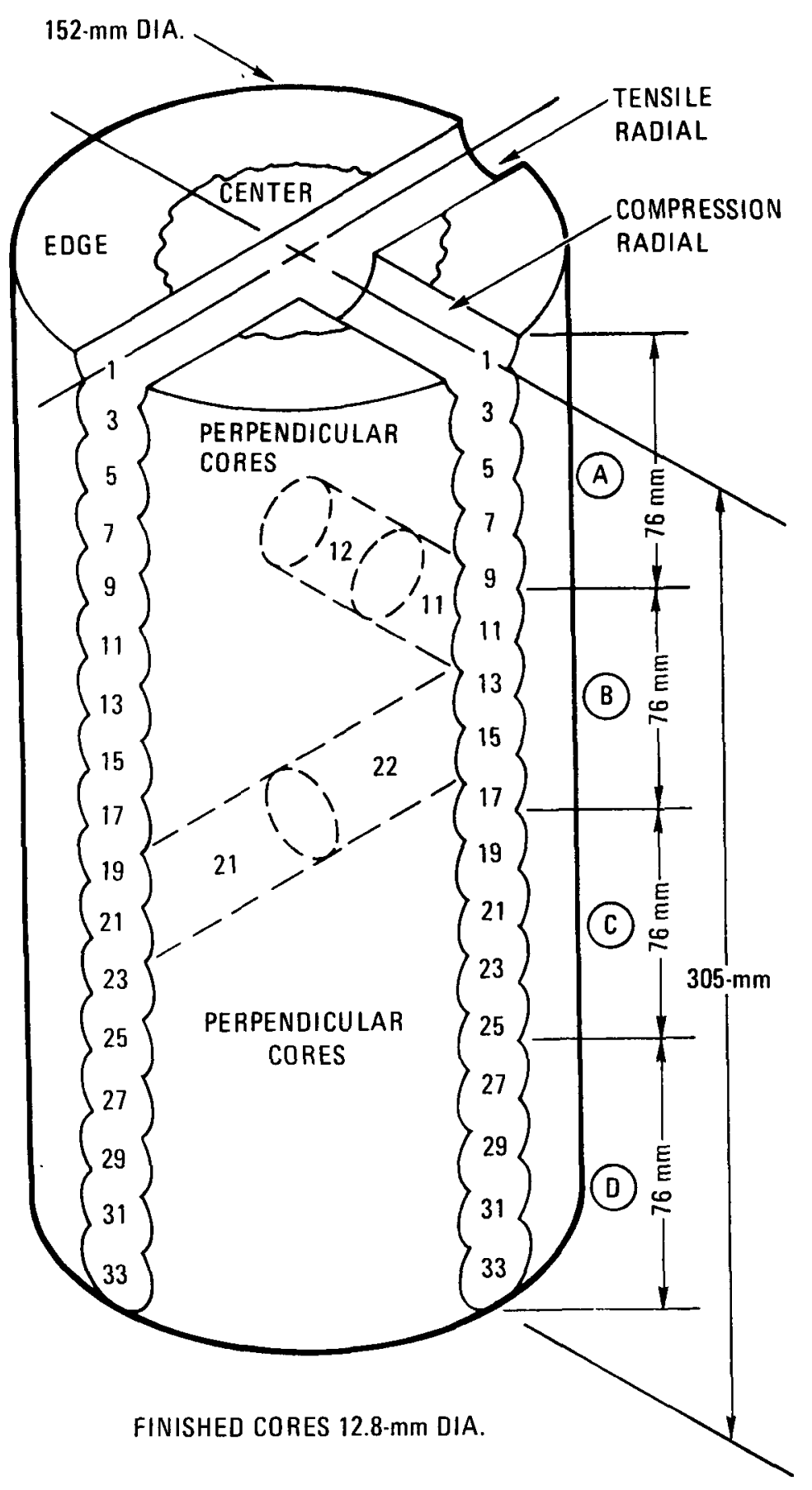

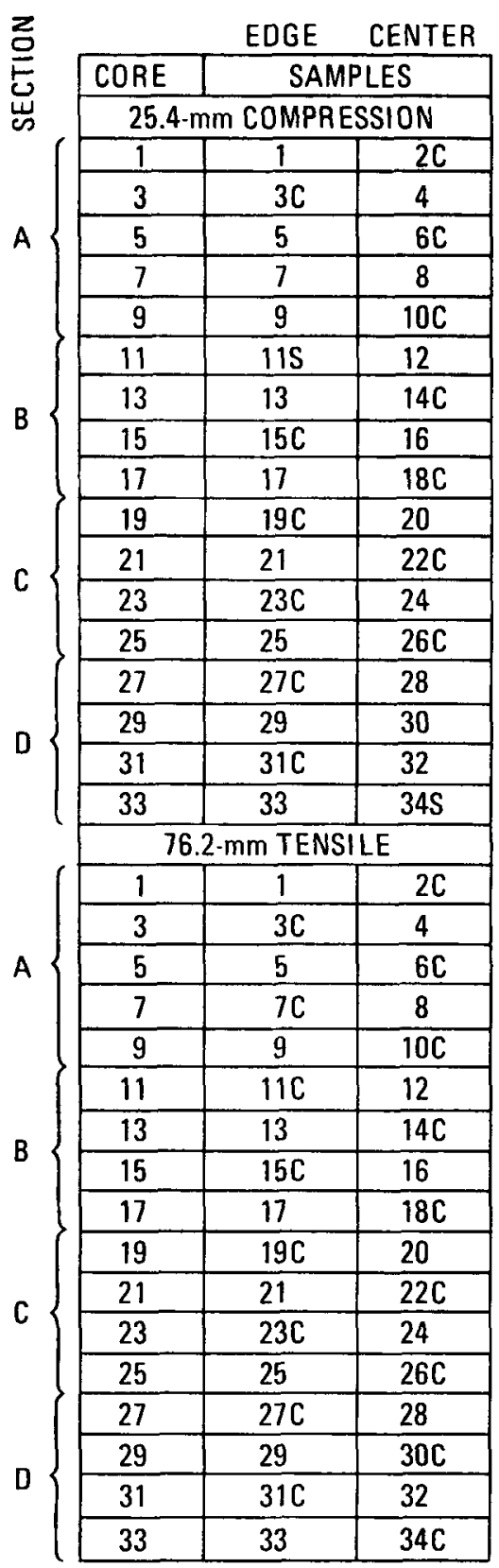

Fig. 5-3(c). Core sampling plan of slab 1 of grade 2020, log 6799-00; radial (core sampling of slabs 3 and 5 was the same as for slab 1) 


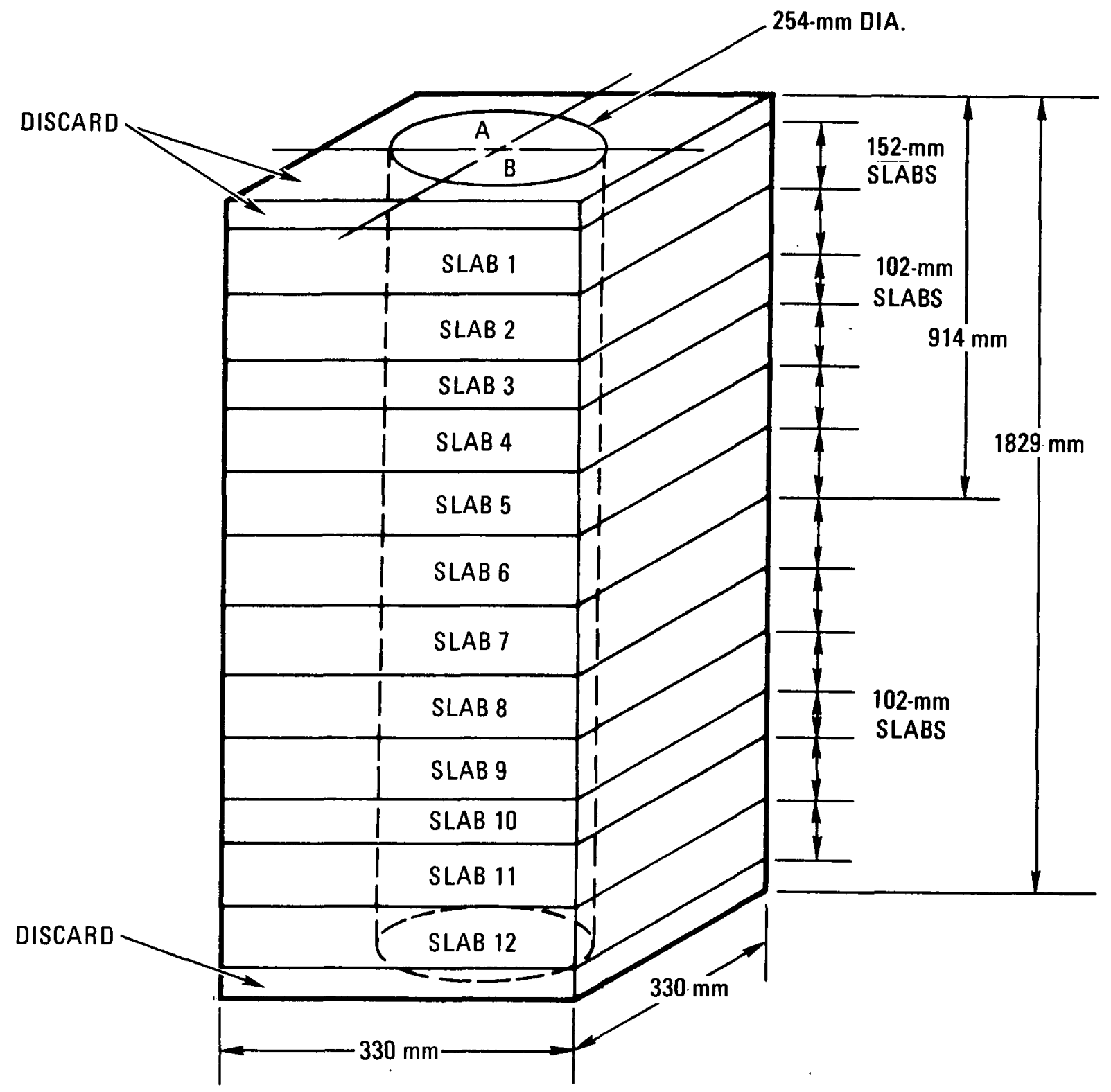

Fig. 5-4. Slab diagram for core support post graphite preproduction grade $\mathrm{H}-440 \mathrm{~N}$ 


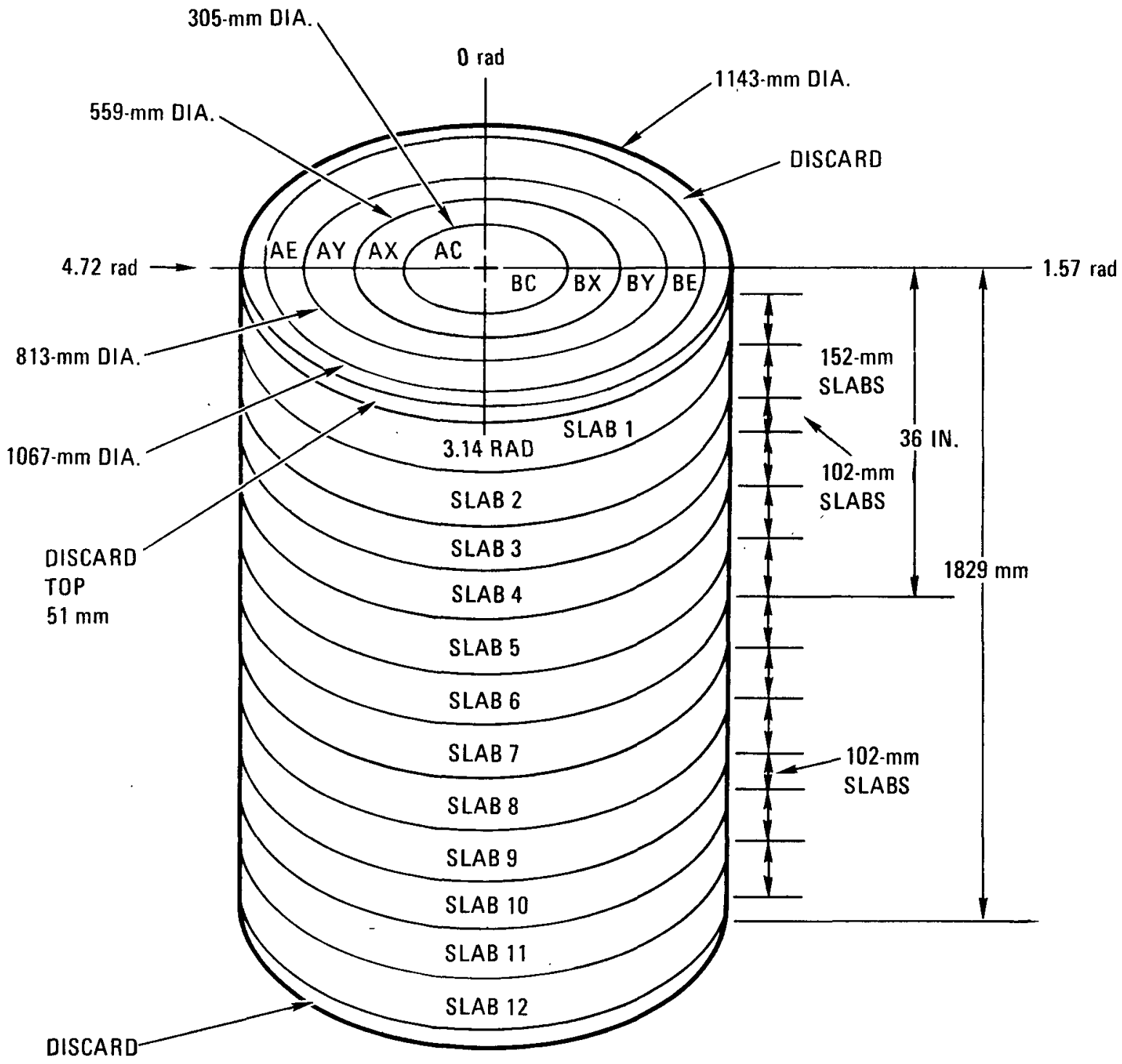

Fig. 5-5(a). Slab diagram for core support floor block and permanent side reflector block graphites (PGX, log 6484-112, or HLM, $\log 6484-78$ ) 


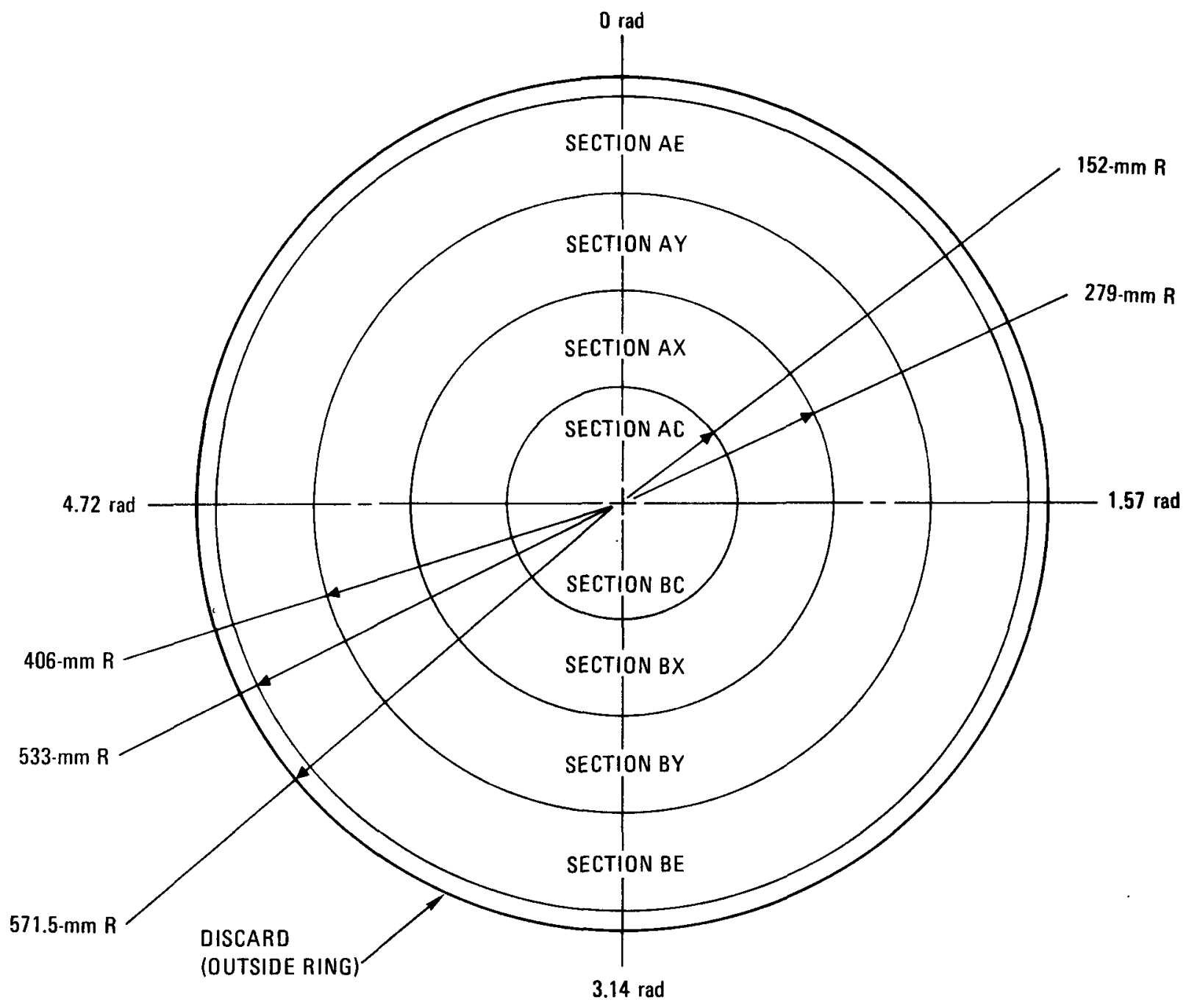

Fig. 5-5(b). Sectioning of core support floor block and permanent side reflector block graphites (PGX, log 6484-112, or HLM, log 6484-78) 


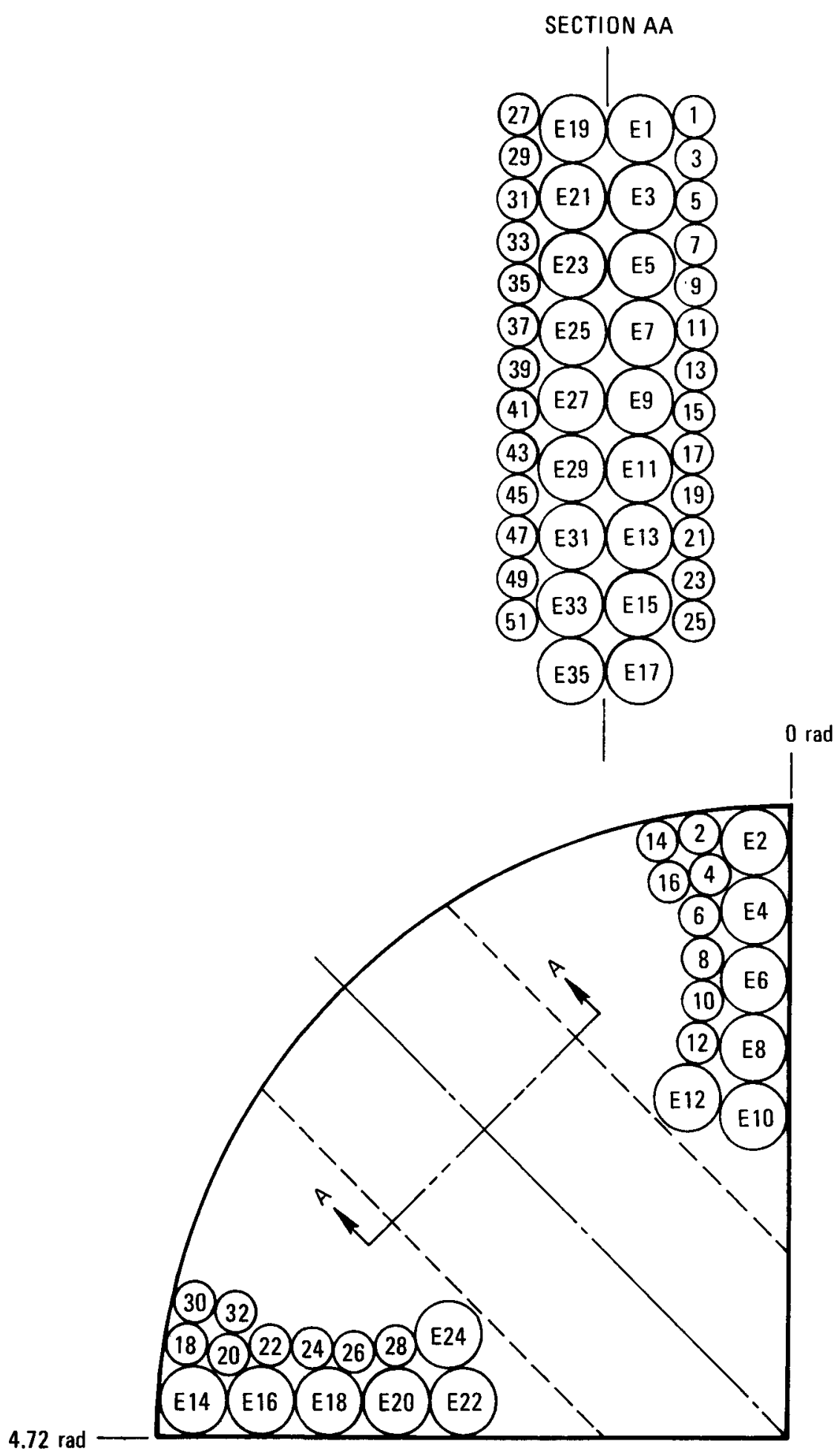

Fig. 5-5(c). Center section: AC 


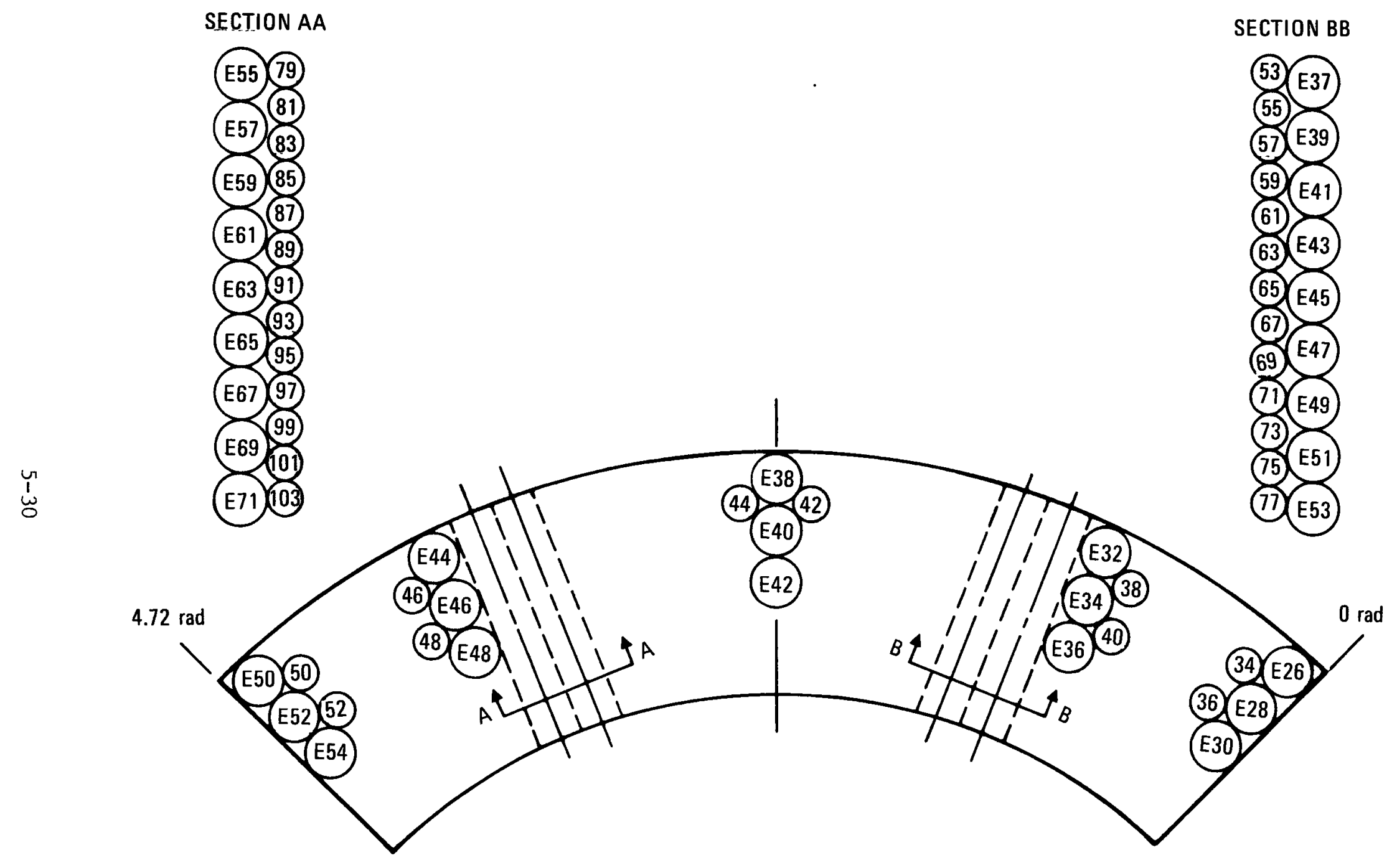

Fig. 5-5(d). Middle section: AY 


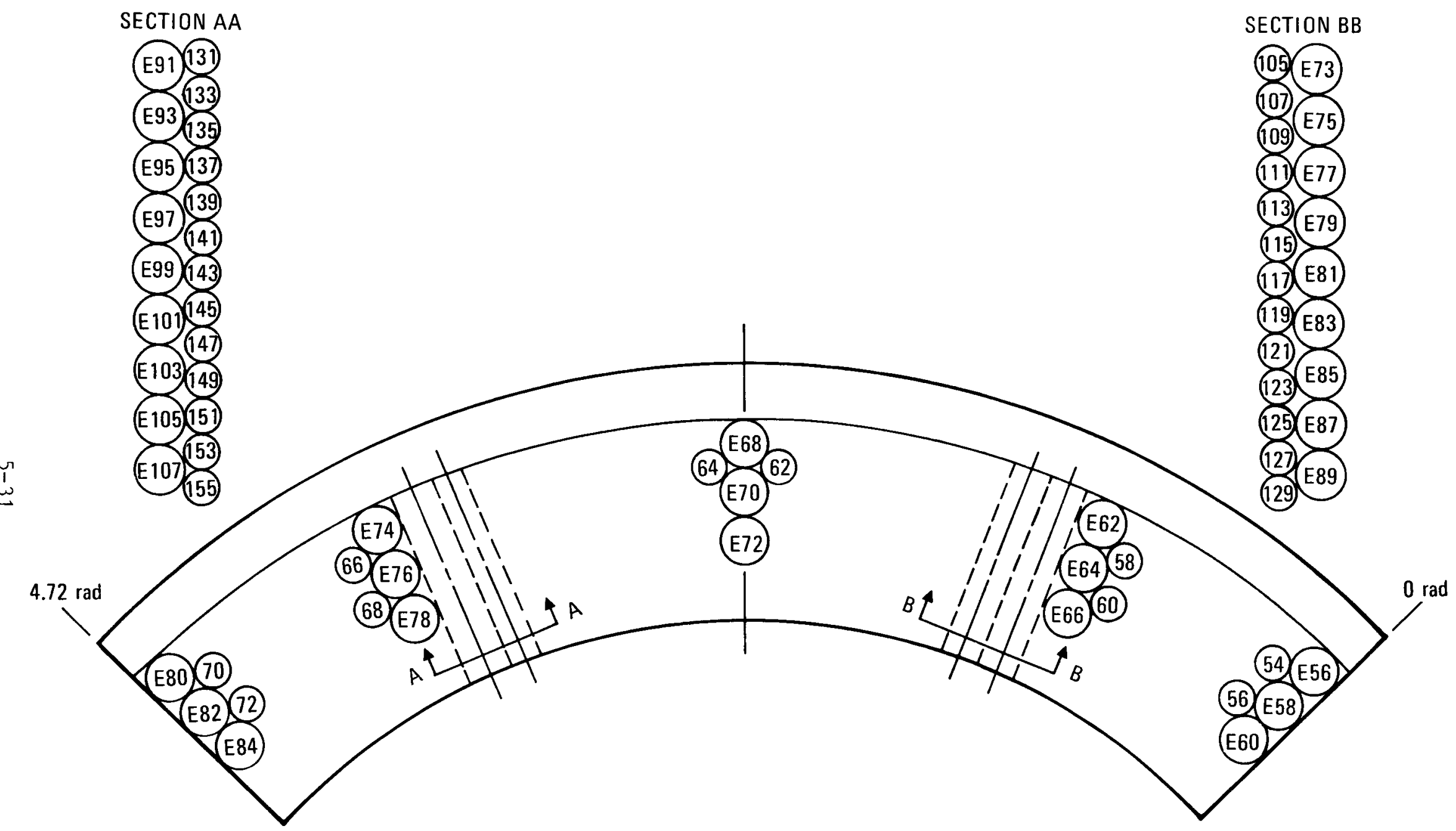

Fig. 5-5(e). Edge section: $\mathrm{AE}$ 


\section{CONCLUSIONS}

Three grades of commercially available candidate graphites -- 2020 for core support posts, PGX for core support floor blocks, and HLM for permanent side reflectors -- have undergone preliminary characterization tests to determine their mechanical properties and chemical impurity contents. An additional experimental grade, $\mathrm{H}-440 \mathrm{~N}$, was also evaluated as a candidate for core support posts. The data were collected in a systematic pattern that revealed property and impurity gradients within the individual logs. As expected, each grade was different with respect to properties and gradients. The data assembled in this report will serve as a portion of the data base required to select reference grades for LHTGR components. Other properties, such as thermal expansivity, thermal conductivity, oxidation resistance, fatigue, and irradiation behavior, which are not reported here will also be considered in the overall evaluation of these graphites. However, the data sets reported here will be analyzed and used initially to determine tentative design configurations and to calculate allowable stress margins. 


\section{ACKNOWLEDGMENTS}

The author expresses thanks to C. H. Richards, J. R. Whaley, M. G. Dunlap, F. R. Hogan, and D. W. Stevens for the laboratory work.

L. H. Juel and R. F. Peters of Great Lakes Carbon Corporation, A. E. Goldman of Union Carbide Corporation, and W. A. Nystrom of Stackpole Carbon Company are acknowledged for their cooperation and helpful comments during the course of this work. 


\section{REFERENCES}

1. Johnson, W. R., and G. B. Engle, "Properties of Unirradiated Fuel Element Graphites H-451 and TS-1240," ERDA Report GA-A13752, General Atomic Company, January 31, 1976.

2. Engle, G. B., and W. R. Johnson, "Properties of Unirradiated Fuel Element Graphites H-451 and S0818," ERDA Report GA-A14068, General Atomic Company, October 8, 1976.

3. Engle, G. B., et al., "Development Status of Near-Isotropic Graphites for Large HTGRs," USAEC Report GA-A12944, General Atomic Company, June 1, 1974.

4. Price, R. J., and L. A. Beavan, "Final Report on Graphite Irradiation Test OG-3," ERDA Report GA-A14211, General Atomic Company, January 1977.

5. Price, R. J., and L. A. Beavan, "Final Report on Graphite Irradiation Test OG-2," ERDA Report GA-A13556, General Atomic Company, December $15,1975$.

6. Cobb, H. R. W., G. B. Engle, and S. J. S. Perry, "Characterization of Production-Grade H-327 Graphite for HTGR Design," in Proceedings of Conference on Continuum Aspects of Graphite Design, Gatlinburg, Tennessee, November 9-12, 1970, USAEC Report CONF-701105, p. 98. 
APPENDIX A

GRADE 2020 DATA

This appendix contains complete data sets for the bulk density, tensile properties, flexural strength, compressive properties, and chemical impurity content of grade 2020 graphite. 
TABLE A-1

TENSILE PROPERTIES OF 2020 GRAPHITE

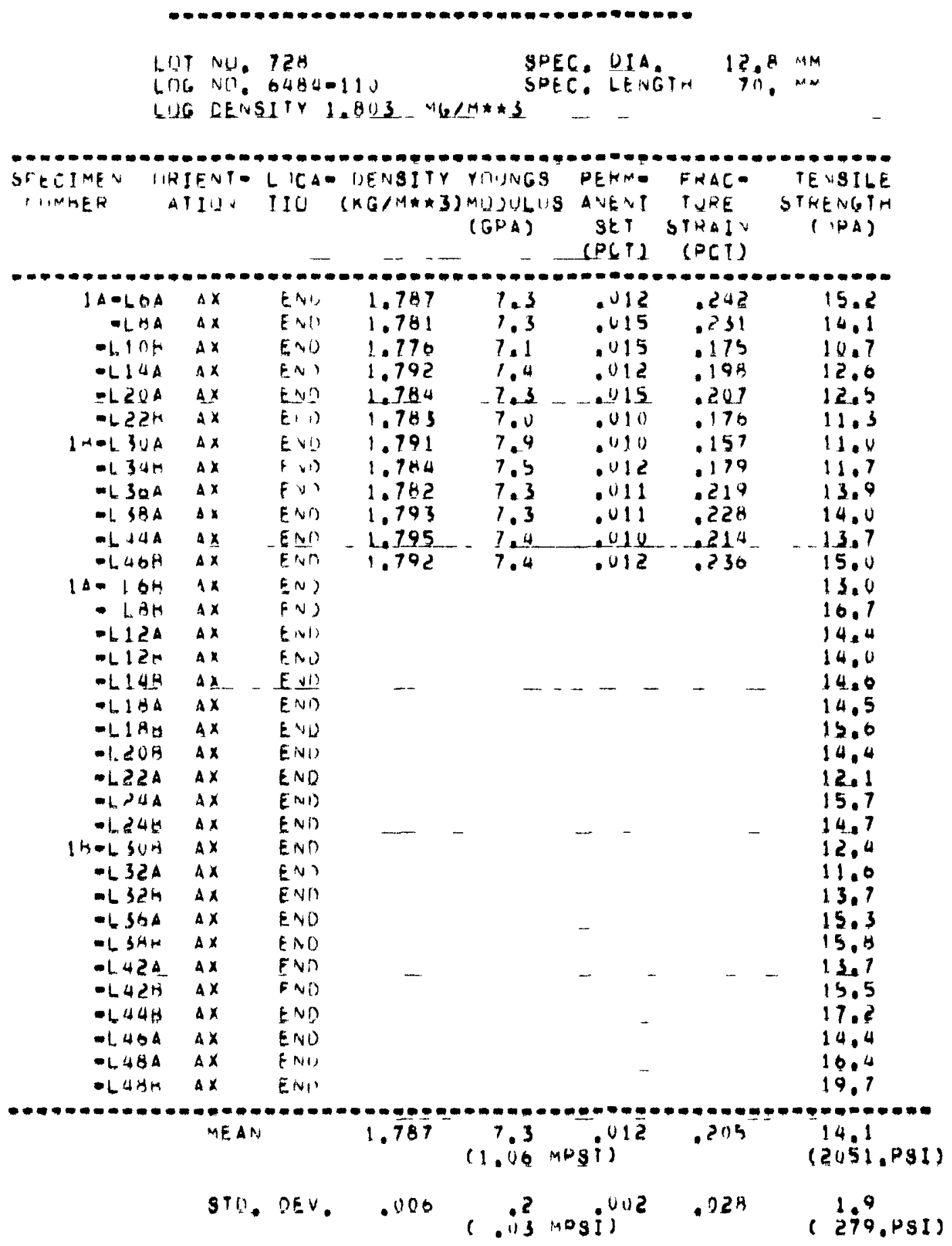

A-2 


\section{TABLE A-1 (Continued)}

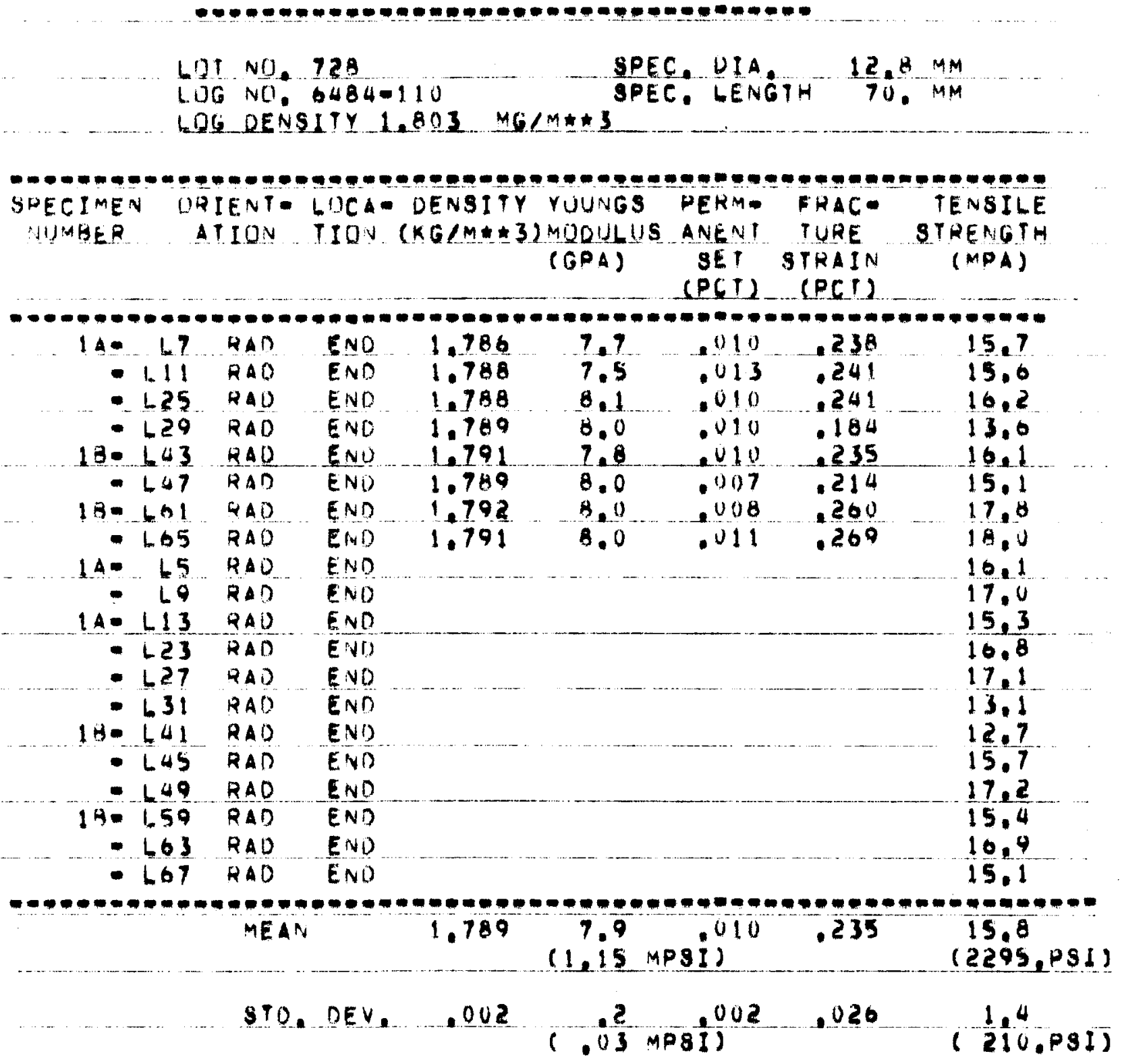


L11 NG. 729 SPEC. DIA. $12.6 \mathrm{MM}$

i If, wi: b4hide11)

LUL UENSIIY 1.6 S MG/M*IS

\begin{tabular}{|c|c|c|c|c|c|c|c|}
\hline $\begin{array}{l}\text { SUECIMEV } \\
Y J A K F\end{array}$ & $\begin{array}{c}\text { WRIF VT } \\
\text { ATIUV }\end{array}$ & $\begin{array}{l}11 C A= \\
11] 2\end{array}$ & $\begin{array}{l}\text { DFASIIY } \\
(A \sqcup I M * 3\end{array}$ & $\begin{array}{l}\text { Y!UNGS } \\
\text { M1DULUS } \\
\text { (GDA) }\end{array}$ & $\begin{array}{c}\text { DEKM } \\
\text { AVEPI } \\
\text { SET }\end{array}$ & $\begin{array}{l}\text { FWAC= } \\
\text { T1RE } \\
\text { STAAIV }\end{array}$ & $\begin{array}{l}\text { TENSILE } \\
\text { STRENGIH } \\
\text { (MDA) }\end{array}$ \\
\hline & & & & - & (PCT) & $(\rho) I)$ & \\
\hline
\end{tabular}

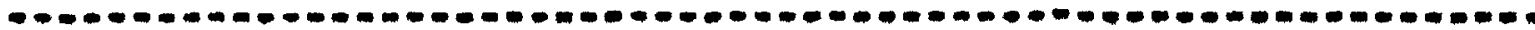

- LSOA AX

$-6 \operatorname{lom} A x$

- Lo? $D$ a

$-\log \Delta \leq x$

$-1.71)^{4} \quad A x$

$64-17 A 4 \quad 1 X$

- Ldem ax

- Leisa ax

- Laga ax

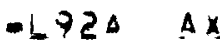

$-194 \pi \quad \Delta x$

$D A=\operatorname{LS\cap A} \quad A x$

$-15)$ H $\quad a x$

-154 h $a x$

- 1 5om $a x$

- Lova a

- 1.bua ax

$-1 \cos A x$

- Inota ax

- Lgati $\Delta x$

- Ljua ax

$-1.7 ? a \quad A x$

$-472 n \quad \Delta x$

$0 B-174 \Delta \quad A x$

$-17 \int K \quad A X$

- L78B Ax

- Lina ax

$-\operatorname{lgux} a x$

- Lath ax

- Inom ax

$-100 \Delta \quad A x$

- Loun $A x$

- $1.92 \pi \quad \Delta x$

$-1.94 a$ ax

$-6.96 a$ a $x$

- $690 \mathrm{~m}$

$\begin{array}{lll}M L & 1.795 & 7.0 \\ M L & 1.774 & 0.9 \\ H L & 1.775 & 0.8 \\ H L & 1.794 & 7.2 \\ M L & 1.793 & 7.2 \\ M L & 1.792 & 7.2 \\ M L & 1.795 & 7.4 \\ M L & 1.791 & 0.6 \\ " L & 1.781 & 0.9 \\ M L & 1.794 & 7.6 \\ M L & 1.943 & 7.2 \\ M L & 1.798 & 7.3\end{array}$

.01

20h

.259

12.7

.9

. U10

.260

14.1

7. 2

. 111

2i14

15.1

. $ง 13$

.204

12.9

410

.230

15.0

- "1"

- 226

.014

.239

- 111

.217

- viz

145

- 110

- 25!

.111 .224

14.8

"L

$\rightarrow L$

${ }^{M} \mathrm{~L}$

4

91

"1L

$m L$

$n$

$\because 1$

$A$

"

it

14

ML

$M L$

ils

$M L$

41

ML

$N$

${ }^{4} L$

$M$

$M L$

Mi.

14.3

14.2

13. 3

9.7

15.2

14.1

13.5

11.6

15.5

13.0

17.4

17.6

15.4

$18 \cdot 3$

16.0

15.4

18.4

19.2

1.5

20.2

10.1

17.1

10.4

14.2

15.4

17.2

19.1

12.3

19.0

16.5

18.1

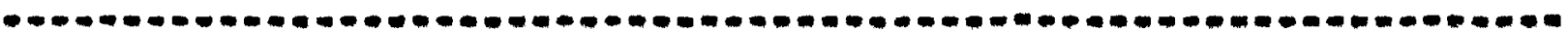

$$
\begin{aligned}
& \text { MEN } \\
& 1.790 \\
& 7.1 \\
& \text {. 2दा } \\
& 13.8 \\
& \text { (1.03 MPSi) } \\
& \text { (2280.PSI) }
\end{aligned}
$$

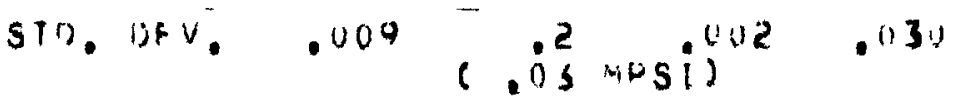

$$
\begin{aligned}
& 2.4 \\
& \text { (354.PSI) }
\end{aligned}
$$

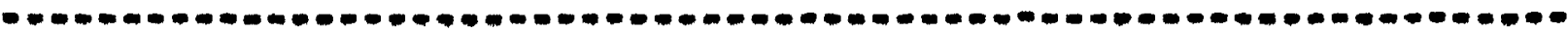




\section{TABLE A-1 (Continued)}

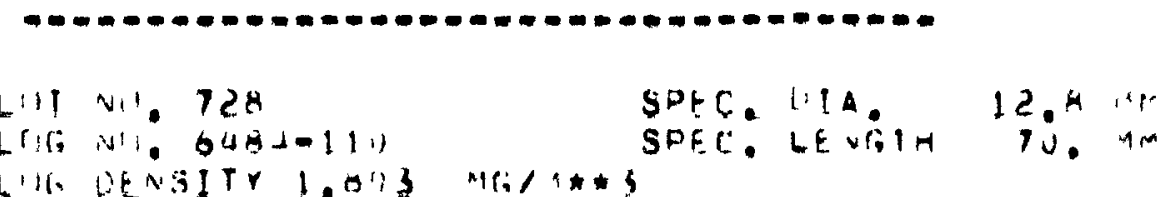

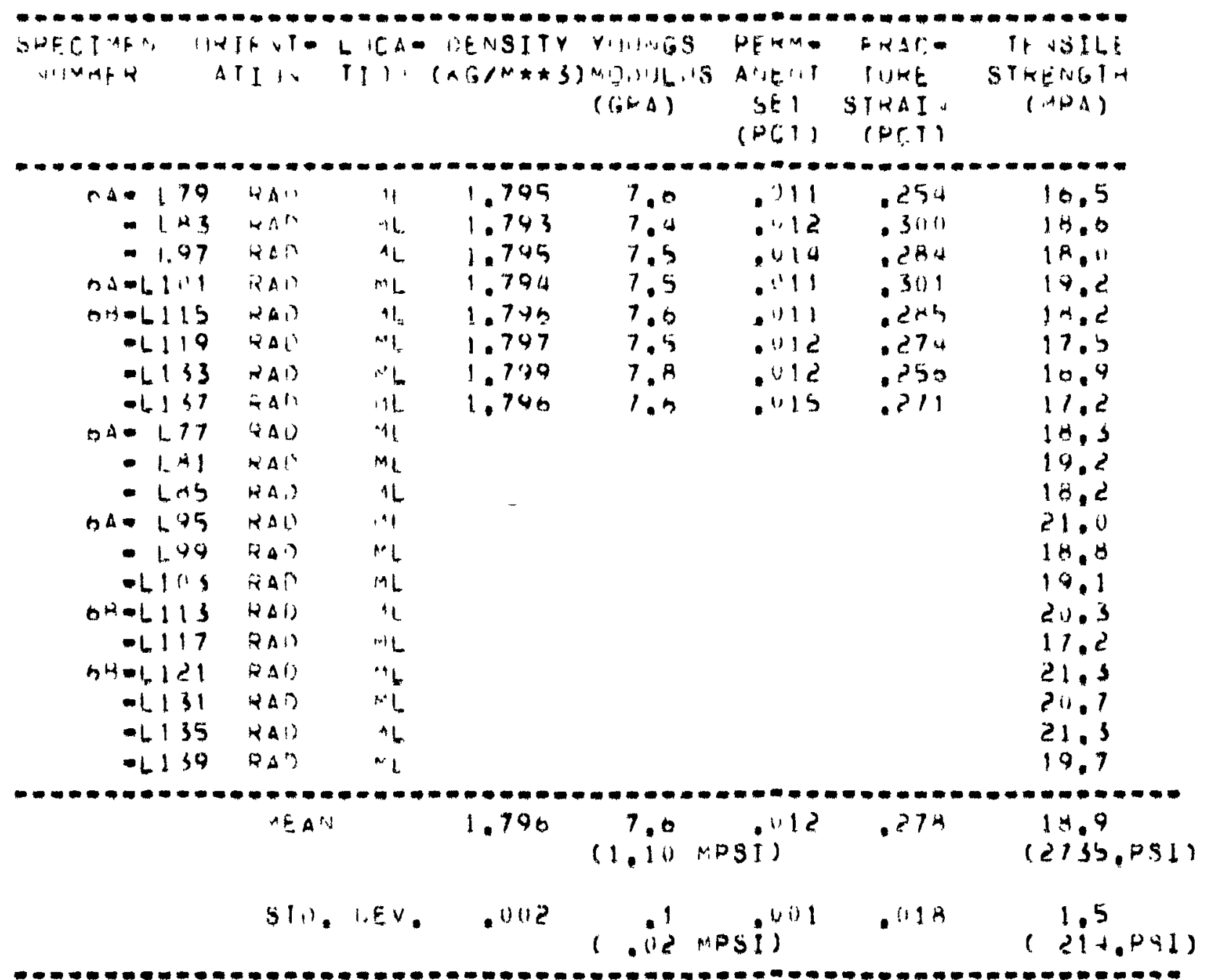


TABLE A-2

TENSILE PROPERTIES OF 2020 GRAPHITE

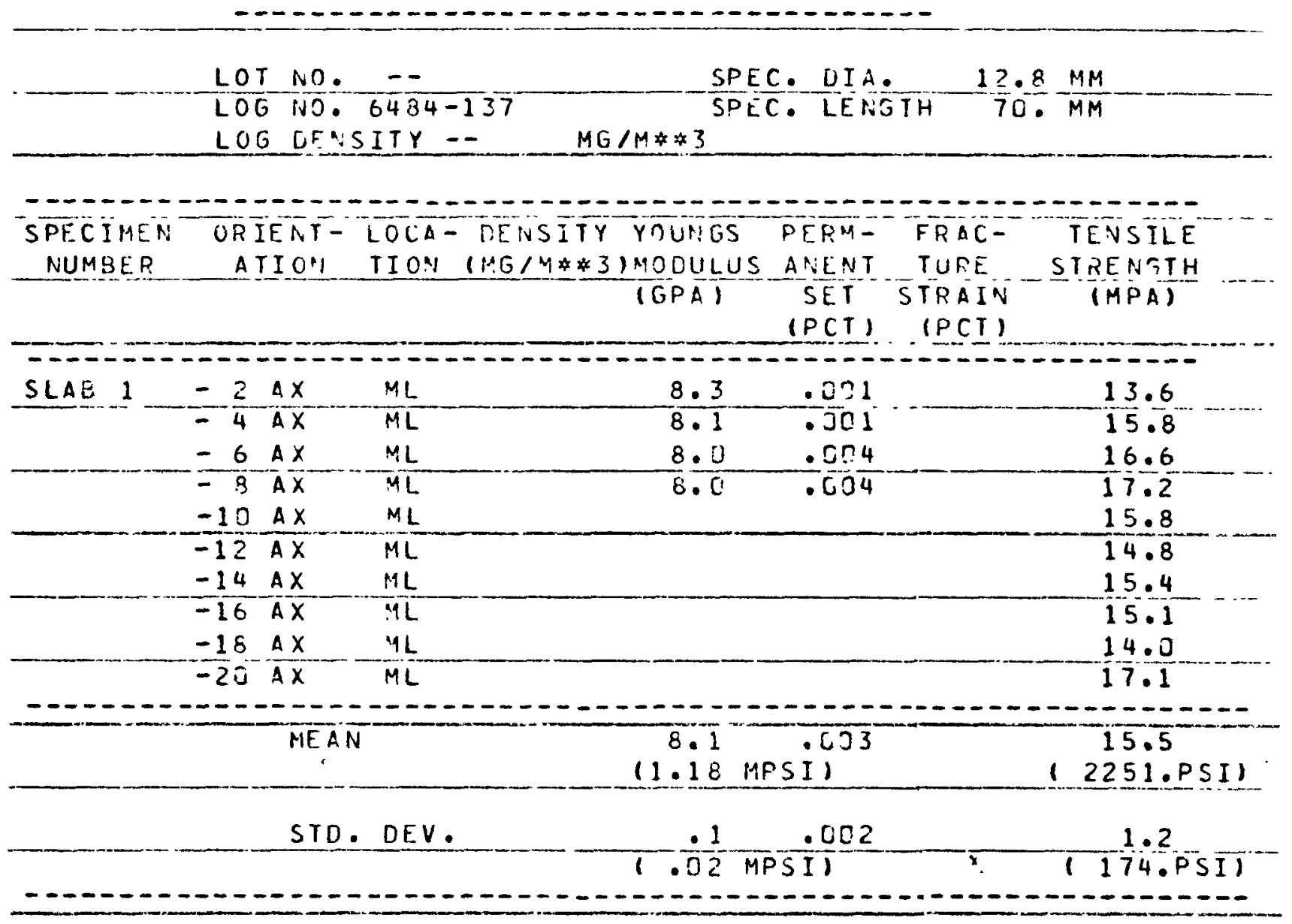


TABLE A-2 (Continued)

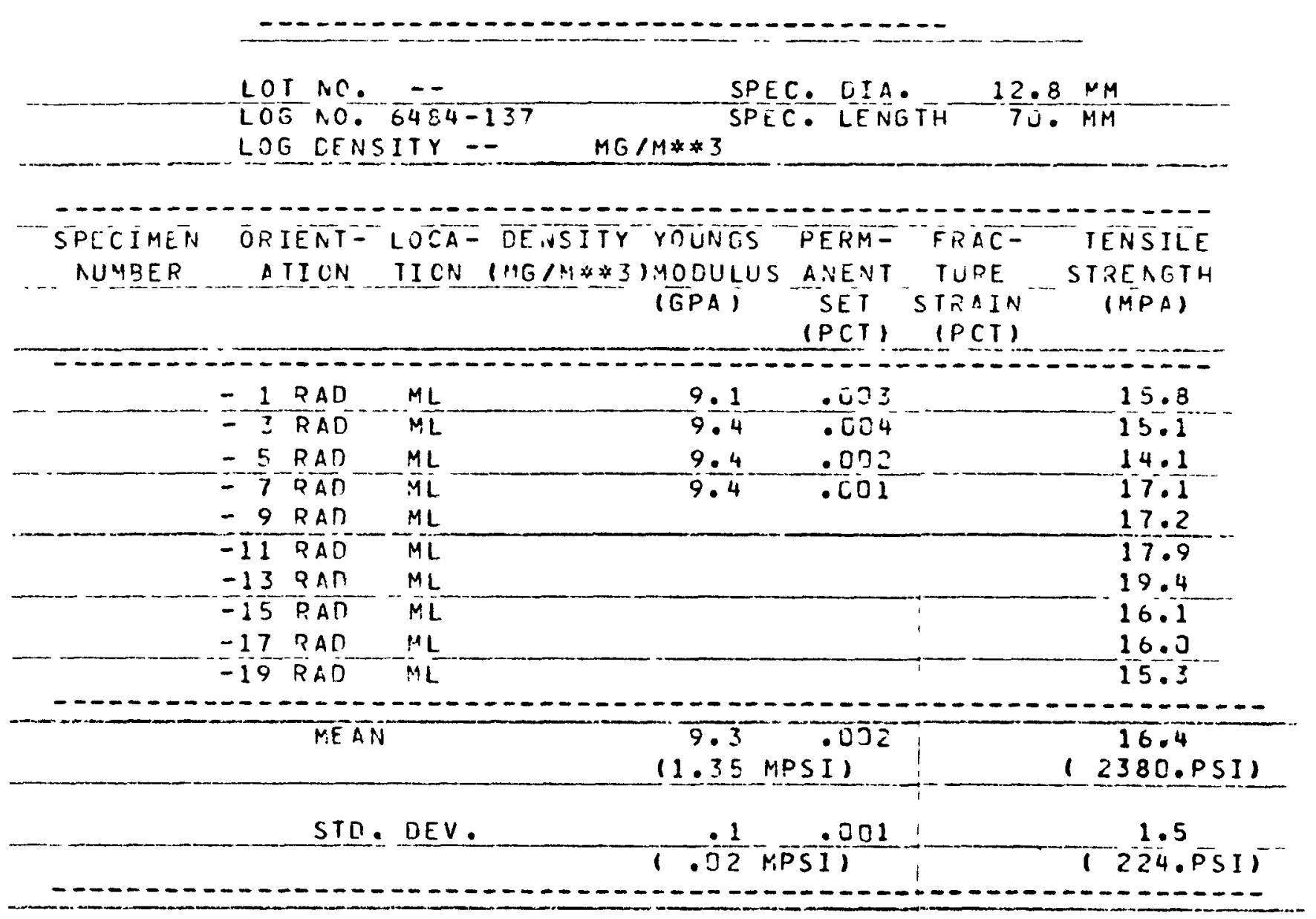


TABLE A-2 (Continued)

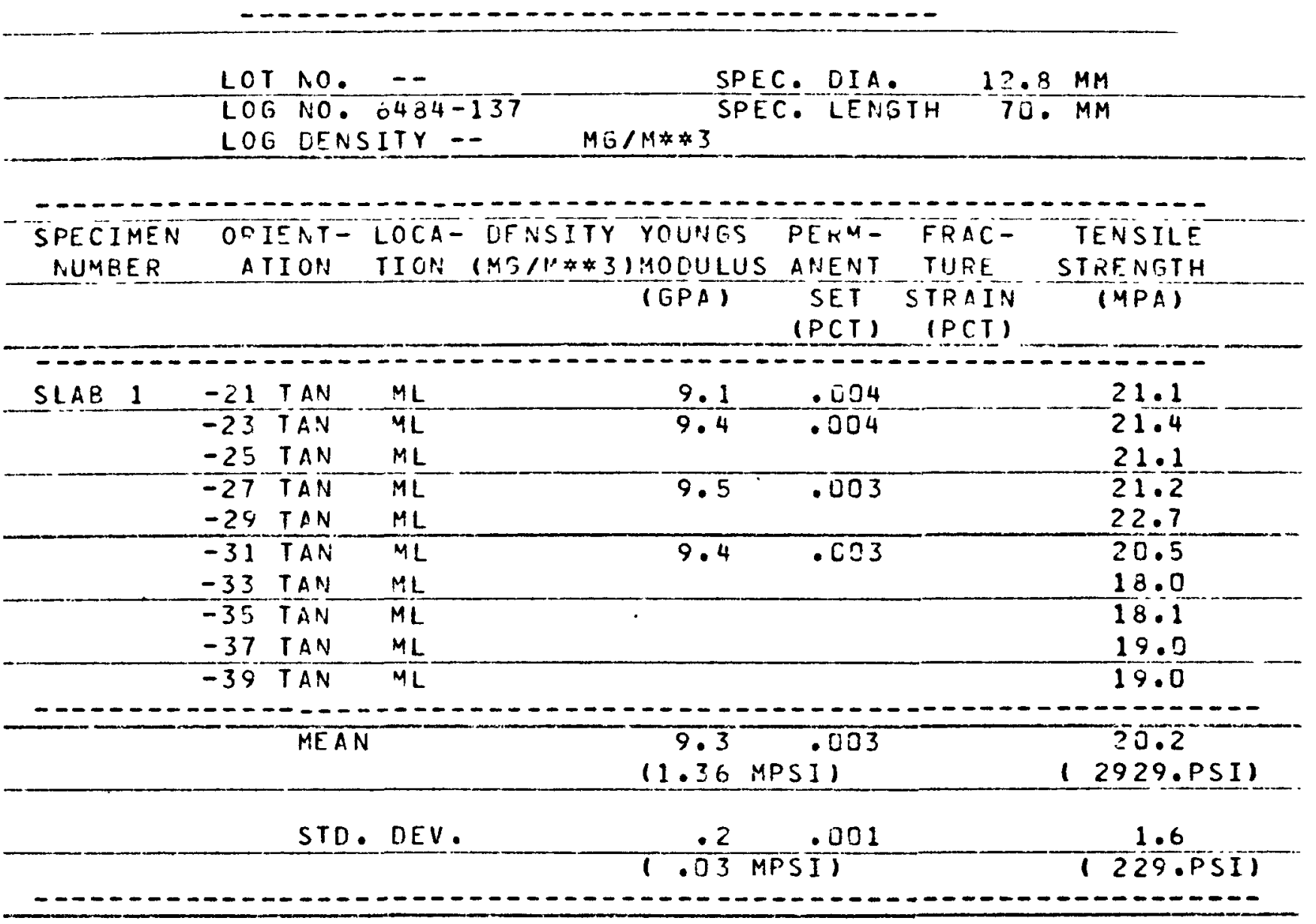


TABLE A-2 (Continued)

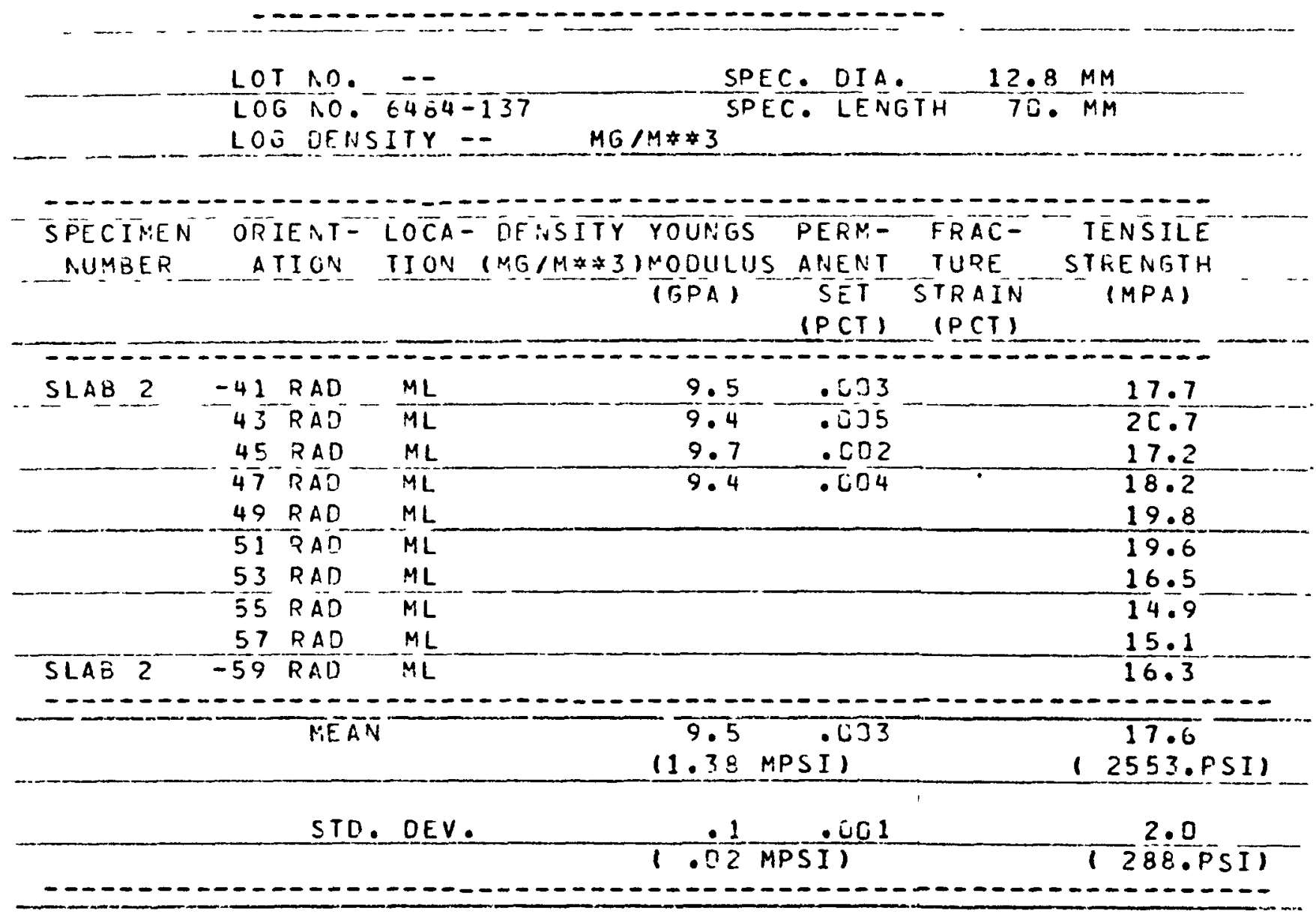


TABLE A-2 (Continued)

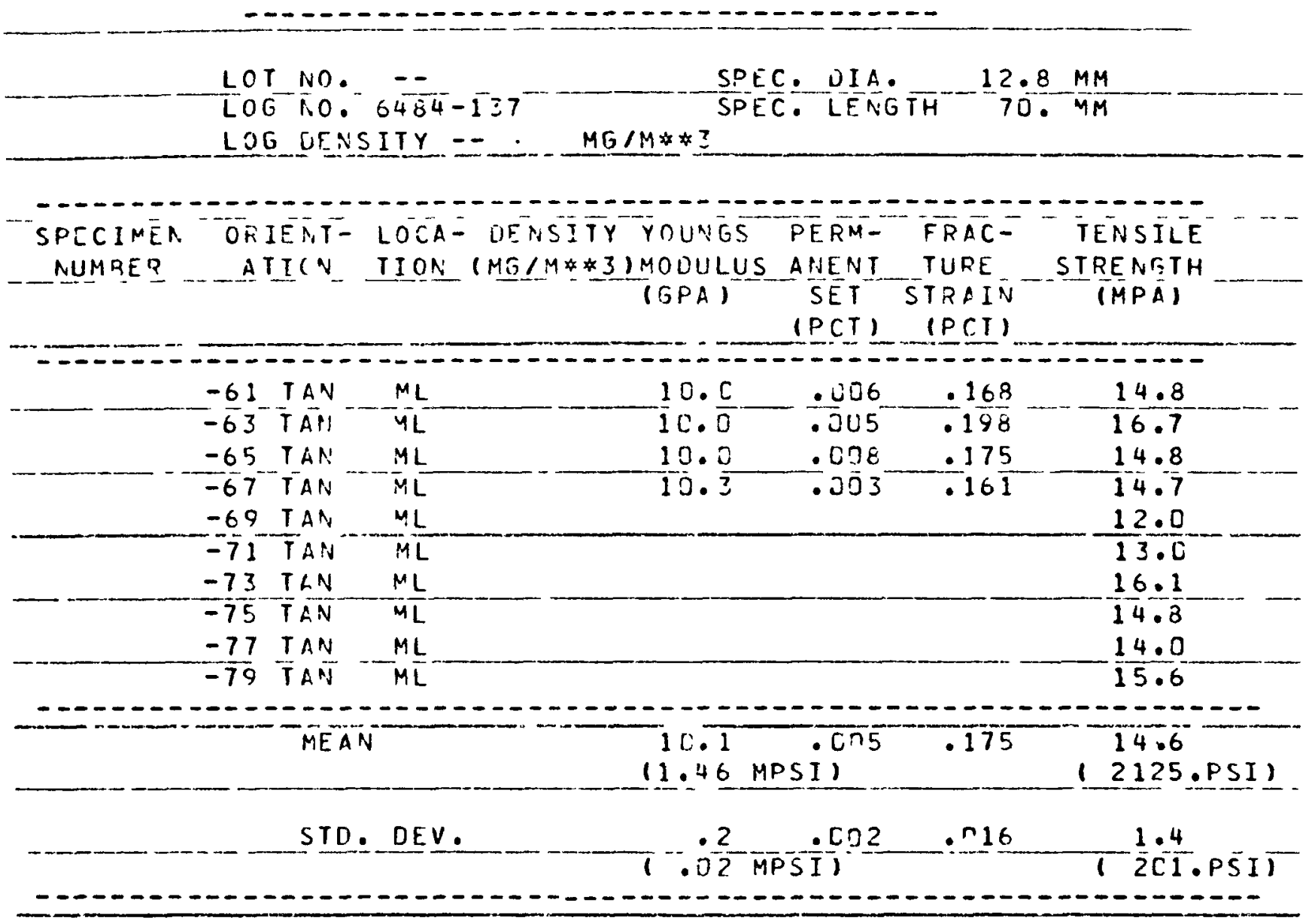


TABLE A-3

TENSILE PROPERTIES OF GRADE 2020 GRAPHITE (LOG 6799-00)

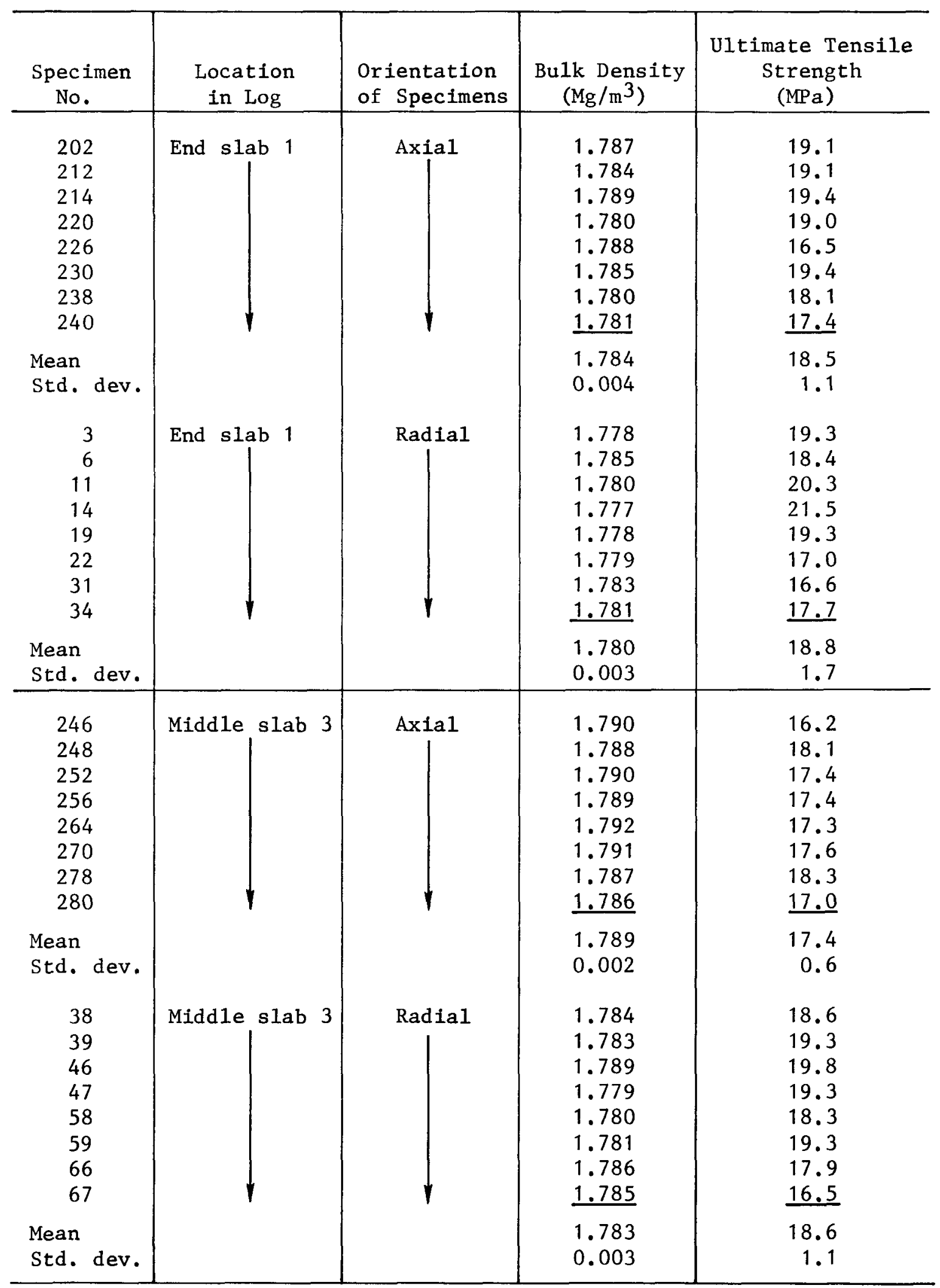


TABLE A-3 (Continued)

\begin{tabular}{|c|c|c|c|c|}
\hline $\begin{array}{l}\text { Specimen } \\
\text { No. }\end{array}$ & $\begin{array}{l}\text { Location } \\
\text { in Log }\end{array}$ & $\begin{array}{l}\text { Orientation } \\
\text { of Specimens }\end{array}$ & $\begin{array}{c}\text { Bulk Density } \\
\left(\mathrm{Mg} / \mathrm{m}^{3}\right)\end{array}$ & $\begin{array}{c}\text { Ultimate Tensile } \\
\text { Strength } \\
(\mathrm{MPa})\end{array}$ \\
\hline $\begin{array}{l}284 \\
290 \\
296 \\
300 \\
306 \\
310 \\
312 \\
316\end{array}$ & End slab 5 & $\stackrel{\text { Axial }}{\mid}$ & $\begin{array}{l}1.801 \\
1.798 \\
1.799 \\
1.798 \\
1.803 \\
1.802 \\
1.803 \\
1.803 \\
\end{array}$ & $\begin{array}{l}18.1 \\
18.6 \\
18.6 \\
19.0 \\
17.1 \\
20.0 \\
16.2 \\
17.6 \\
\end{array}$ \\
\hline $\begin{array}{l}\text { Mean } \\
\text { Std. dev. }\end{array}$ & & & $\begin{array}{l}1.799 \\
0.005\end{array}$ & $\begin{array}{r}18.2 \\
1.2\end{array}$ \\
\hline $\begin{array}{l}70 \\
71 \\
78 \\
79 \\
90 \\
91 \\
98 \\
99\end{array}$ & End slab 5 & $\mid$ & $\begin{array}{l}1.802 \\
1.799 \\
1.805 \\
1.801 \\
1.807 \\
1.805 \\
1.812 \\
1.810 \\
\end{array}$ & $\begin{array}{l}19.0 \\
17.1 \\
20.1 \\
20.8 \\
18.4 \\
18.6 \\
18.8 \\
19.6 \\
\end{array}$ \\
\hline $\begin{array}{l}\text { Mean } \\
\text { Std. dev. }\end{array}$ & & & $\begin{array}{l}1.805 \\
0.004\end{array}$ & $\begin{array}{r}19.1 \\
1.1\end{array}$ \\
\hline
\end{tabular}


TABLE A-4

FLEXURAI PROPERTIES OF 2020 GRAPHITE

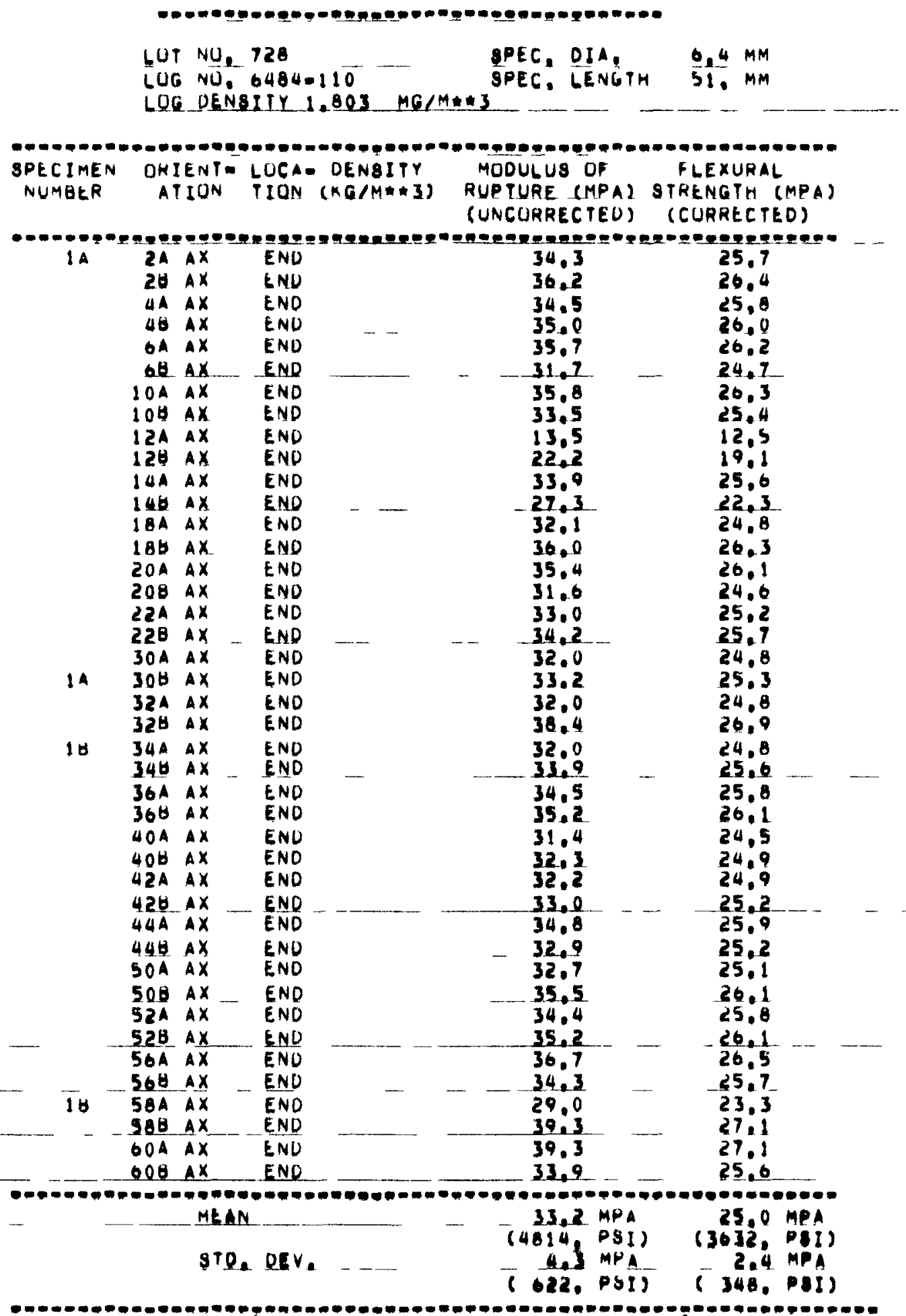


TABLE A-4 (Continued)

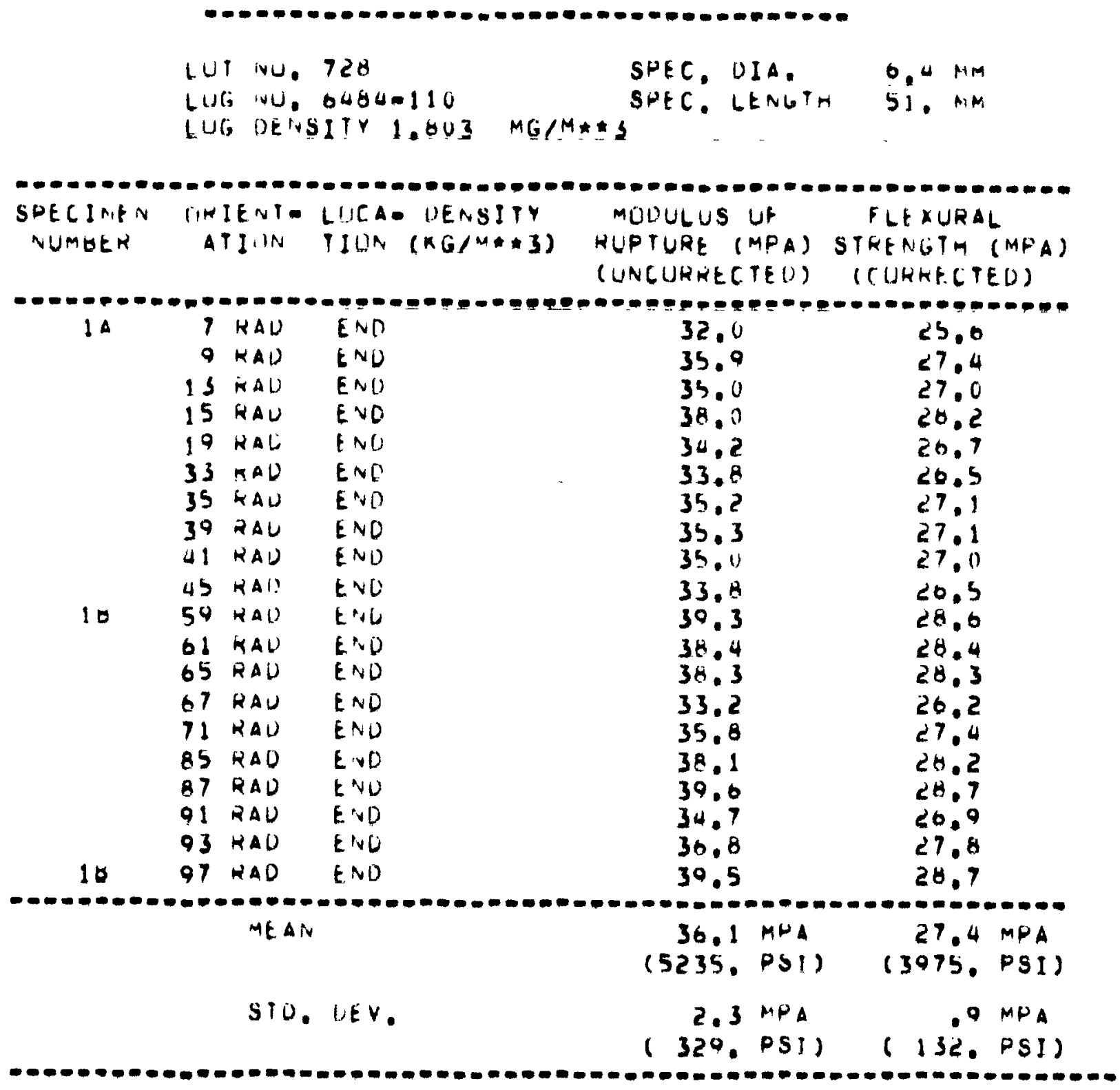


TABLE A-4 (Continued)

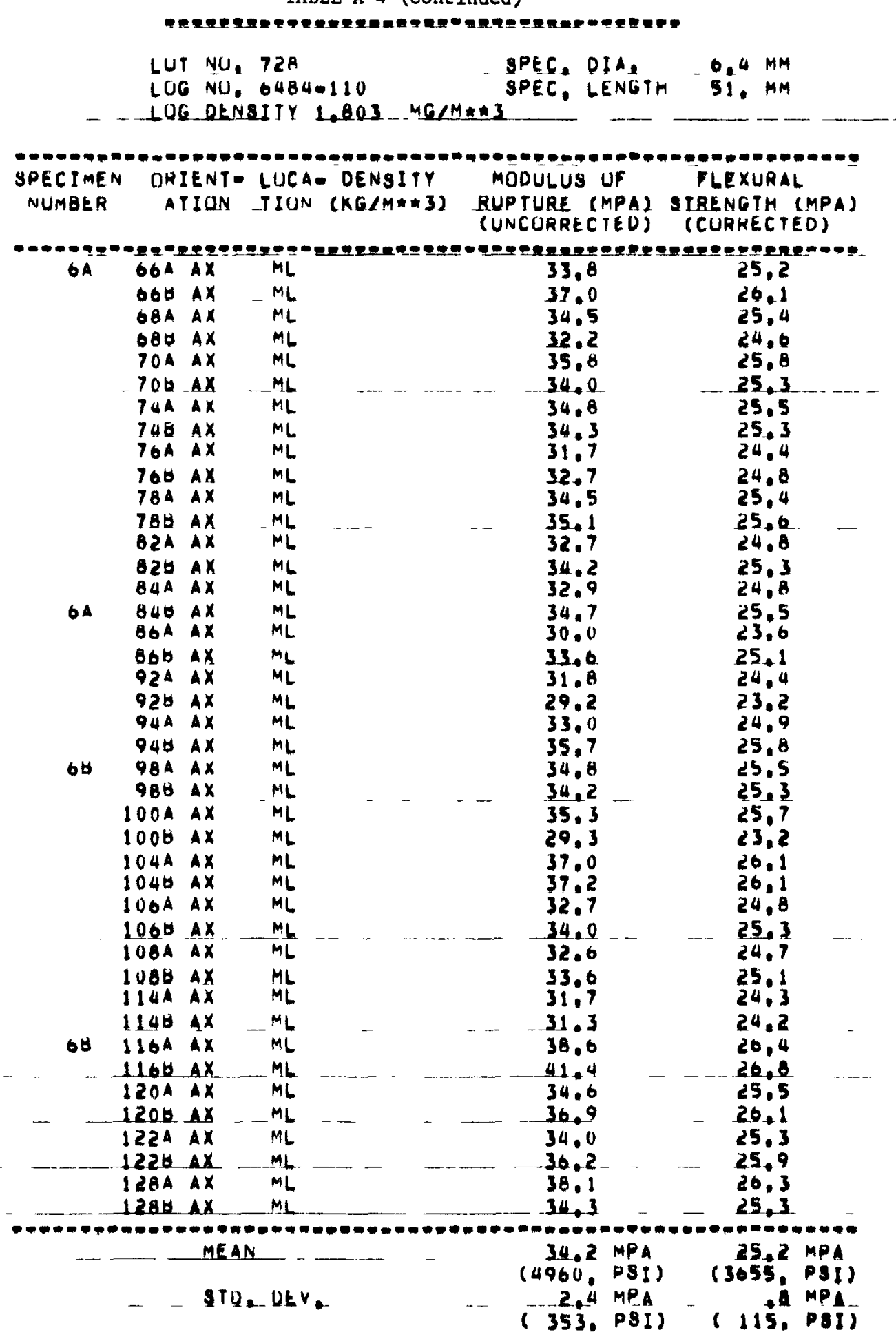

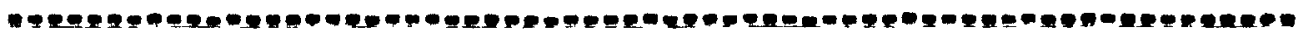


TABLE A-4 (Continued)

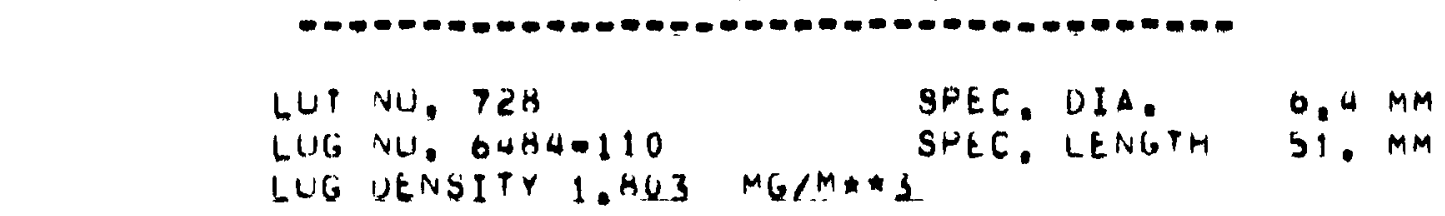

\begin{tabular}{|c|c|c|c|c|c|c|}
\hline $\begin{array}{l}\text { SFECIMEIV } \\
\text { PUUMULR }\end{array}$ & & $\begin{array}{l}\text { IEnI- } \\
\text { IUTh }\end{array}$ & $\begin{array}{l}\text { LUCA } \text { [IENSITY } \\
\text { TION (KG/M**3) }\end{array}$ & $\begin{array}{l}\text { MIJUULUS UF } \\
\text { RUPTURE (MPA) } \\
\text { (UNCURRECIED) }\end{array}$ & $\begin{array}{l}\text { FLEXUKAL } \\
\text { SIRENGTH } \\
\text { ICURKECTE }\end{array}$ & $(M P A)$ \\
\hline nnon & $=0-$ & $\infty-\infty$ & $-2=0$ & 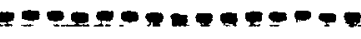 & $0--p=-2=8 x$ & 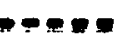 \\
\hline OA & 111 & $K A U$ & $M L$ & 38.4 & 27.7 & \\
\hline & 113 & $K A U$ & $M L$ & 39.0 & 27.9 & \\
\hline & 111 & RAU & $M L$ & 39.5 & 27.9 & \\
\hline & 119 & KAU & $M L$ & 39.7 & 27.9 & \\
\hline & 123 & $K A D$ & $M L$ & 40.6 & 28.1 & \\
\hline & 137 & $K \triangle D$ & $M L$ & 37.3 & 27.3 & \\
\hline & $13^{\circ}$ & RAD & $M L$ & 35.2 & 26.6 & \\
\hline & 143 & KAU & $M_{L}$ & 33.2 & 25.7 & \\
\hline & 145 & HAD & Nil & 35.3 & 26.6 & \\
\hline 60 & 149 & $\begin{array}{l}\text { RAU } \\
\text { RAU }\end{array}$ & $M L$ & 37.0 & $<7.2$ & \\
\hline & $\begin{array}{l}165 \\
169\end{array}$ & $\begin{array}{l}\text { RAD } \\
\text { RAD }\end{array}$ & $\stackrel{M L}{M L}$ & $\begin{array}{l}40.6 \\
37.3\end{array}$ & $\begin{array}{r}28.1 \\
27.3\end{array}$ & \\
\hline & $\begin{array}{l}171 \\
175\end{array}$ & $\begin{array}{l}\text { RAL } \\
\text { RAD }\end{array}$ & $M L$ & $\begin{array}{l}36.9 \\
35.0\end{array}$ & $\begin{array}{l}27.2 \\
20.5\end{array}$ & \\
\hline & 189 & RAL & $M L$ & 30.1 & 20.9 & \\
\hline & 191 & RAD & $M L$ & 39.7 & 27.9 & \\
\hline & 1.95 & RAD & ML & 39.5 & 27.9 & \\
\hline 60 & 197 & KAD & ML & 37.6 & 27.4 & \\
\hline & 201 & RAD & $M L$ & 31.6 & 25.0 & \\
\hline & & $-\infty-\infty$ & 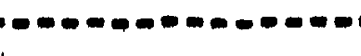 & & & \\
\hline & & $M E A A$ & & 37.3 MPA & 127.2 & MPA \\
\hline & & STU. & WEV. & $2.5 \mathrm{MPA}$ &.$H$ & $M P A$ \\
\hline & & & & $(367 . P S I)$ & 1122. & PSII \\
\hline
\end{tabular}

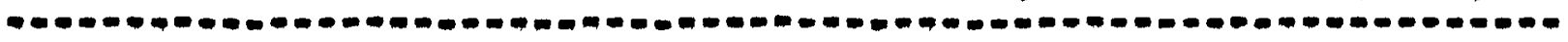


TABLE A-5

COMPRESSIVE PROPERTIES OF 2020 GRAPHITE

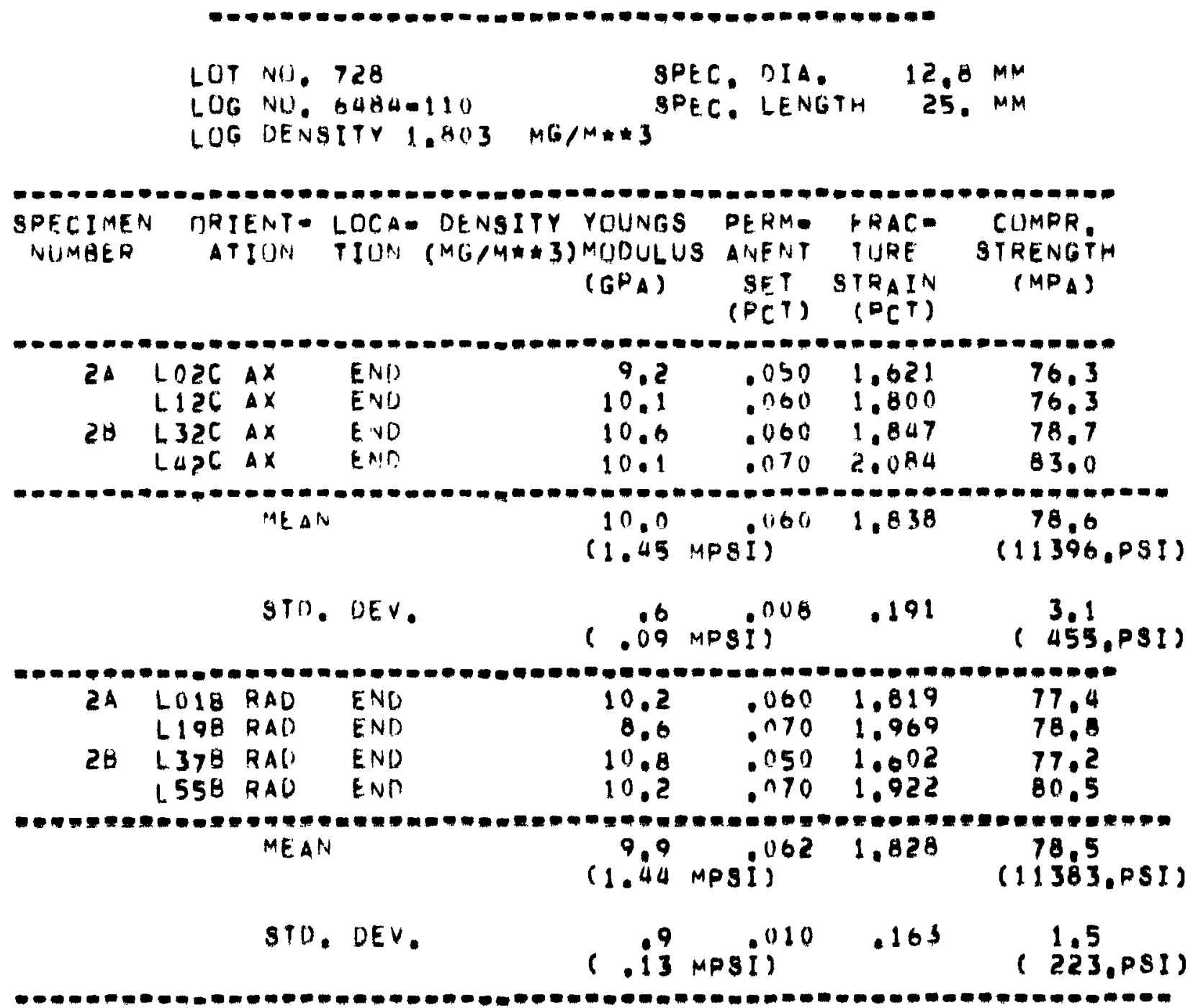


TABLE A-6

COMPRESSIVE PROPERTIES OF 2020 GRAPHITE

- -

LOT NO. - _ SPECE DIA. 12.8 MM

LOG $10.6484-137$ SPEC. LENGTH 23. MM

LOG OENSILY - - MGLM $\$ 3$

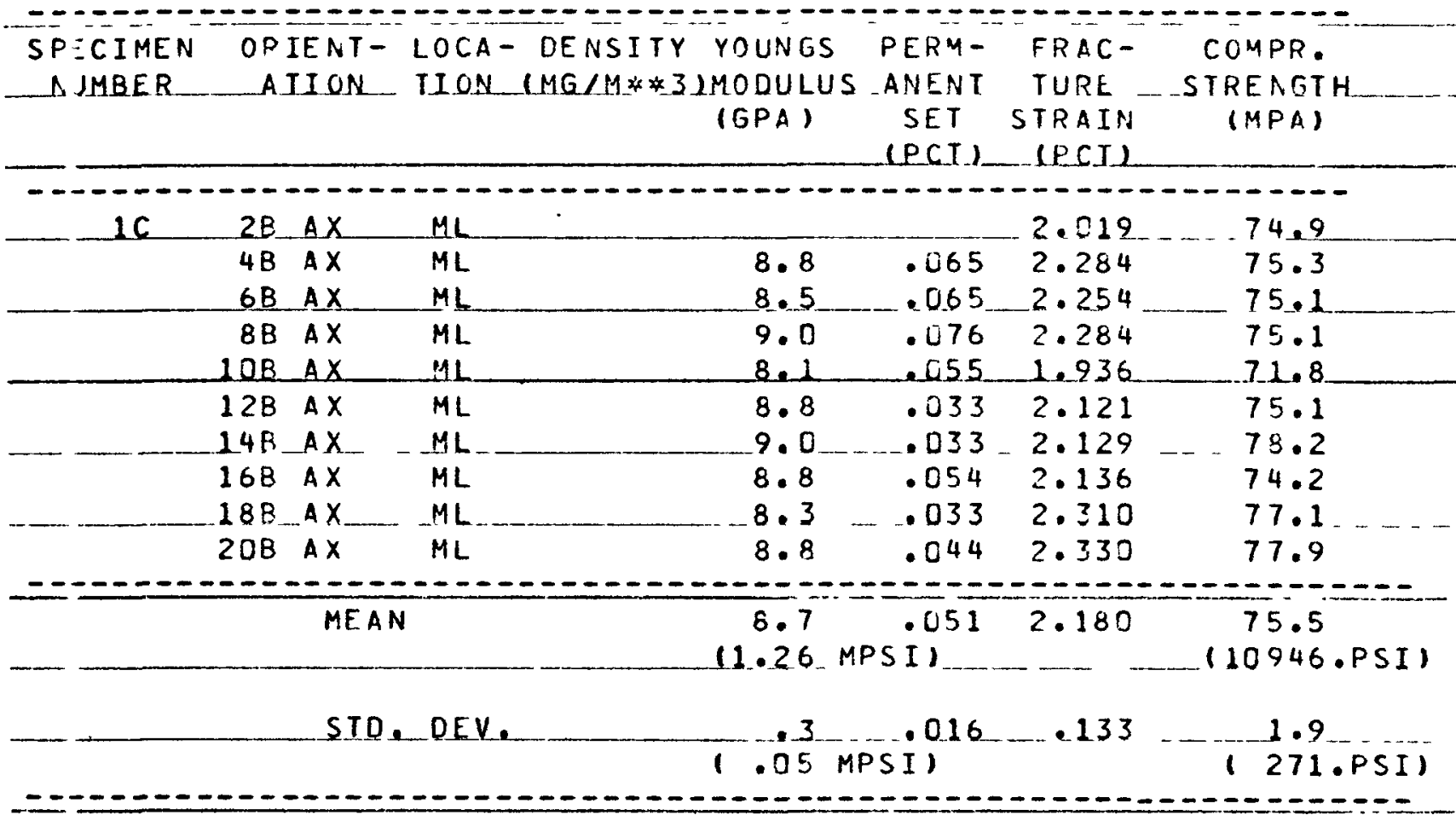


TABLE A-6 (Continued)

$-\cdots-\cdots-\cdots-\cdots-\cdots-\cdots-\cdots-\cdots-\cdots-\cdots$

LOI NO._- - SPEC. DIA. $12.8 \mathrm{MM}$

LOG NO. 6484-137 SPEC. LENGTH 23. MM

LOG DENSITY $==$ MG/M* $* 3$

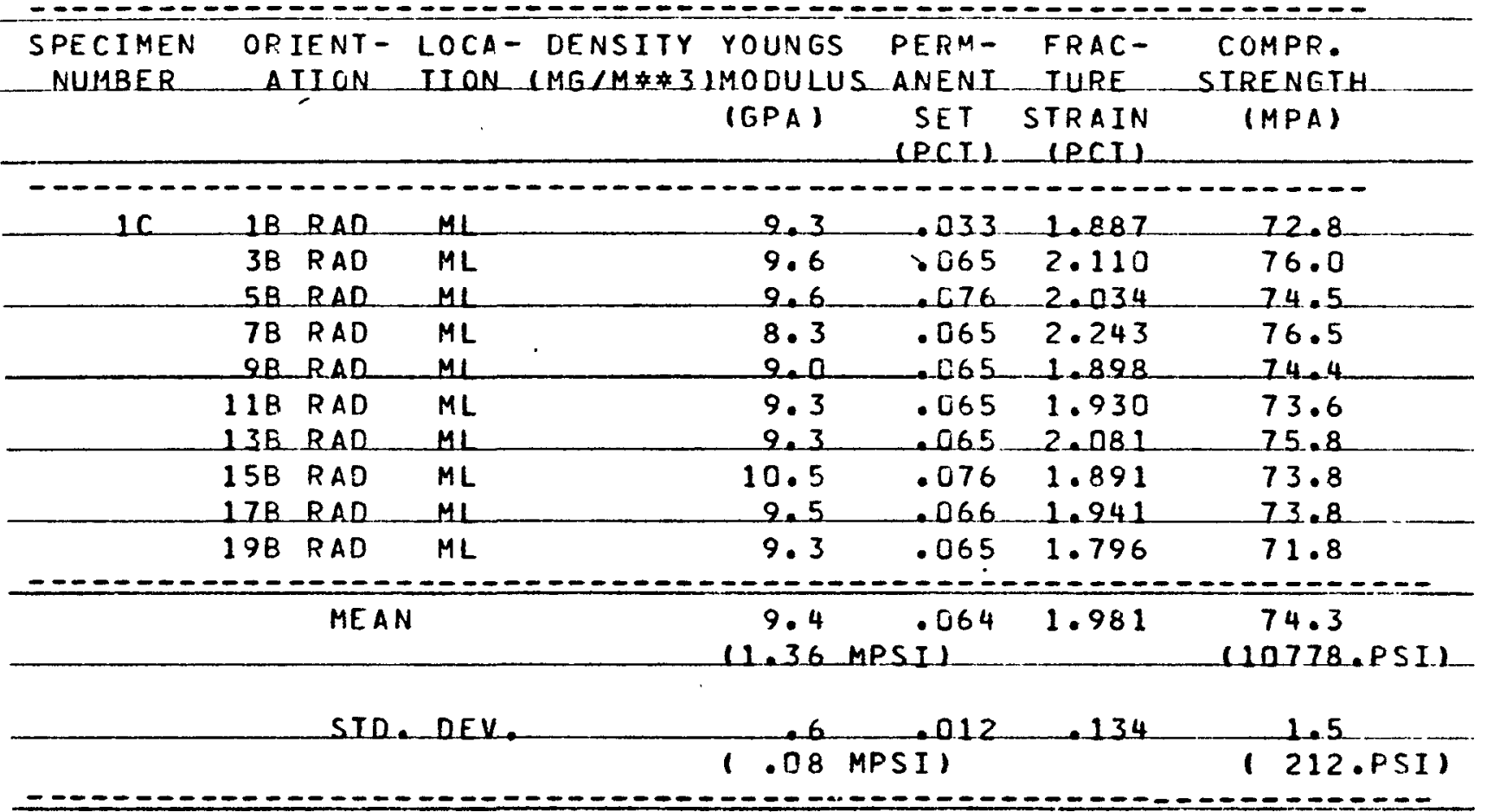


TABLE A-6 (Continued)

--1-1----
LOI NO...-
SPEC. DIA
12.8 MM
LOG NO. $6484-137$
106 DENSIIY --
$M G / M+3$
SPEC. LENGTH
23. $M M$

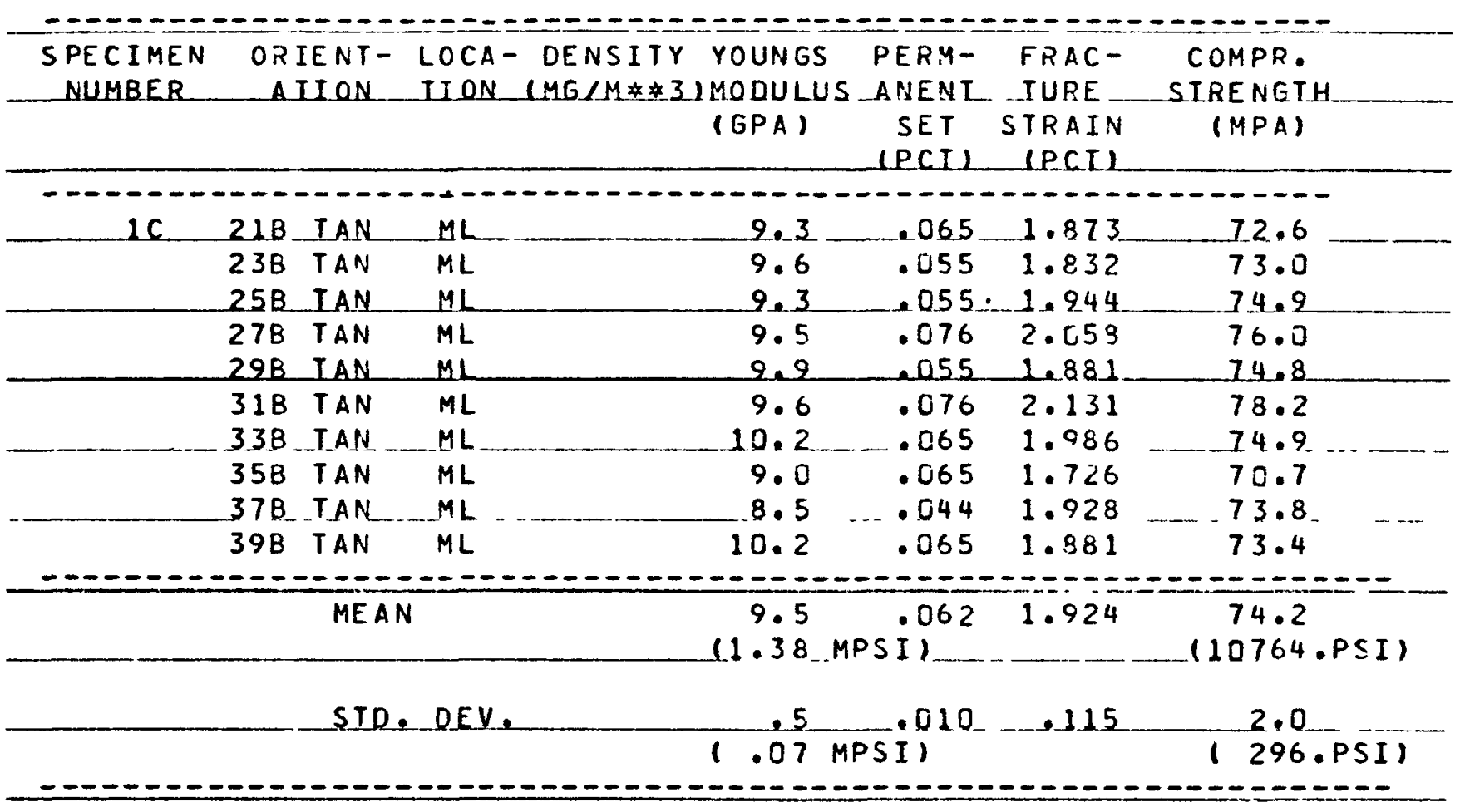


TABLE A-6 (Continued)

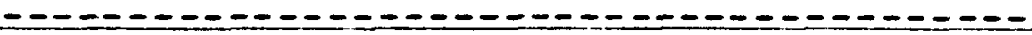

LOINO. - = SP.EC.DIA. $\quad 12.8 \mathrm{MM}$

LOG NO. 6484-137 SPEC. LENGTH 23. MM

LOG CENSIIX - - MG/M* $* 3$

SPECIMEN ORIENT- LOCA- DENSITY YOUNGS PERM- FRAC- COMPR.

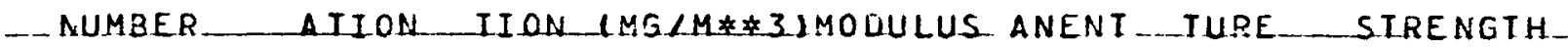

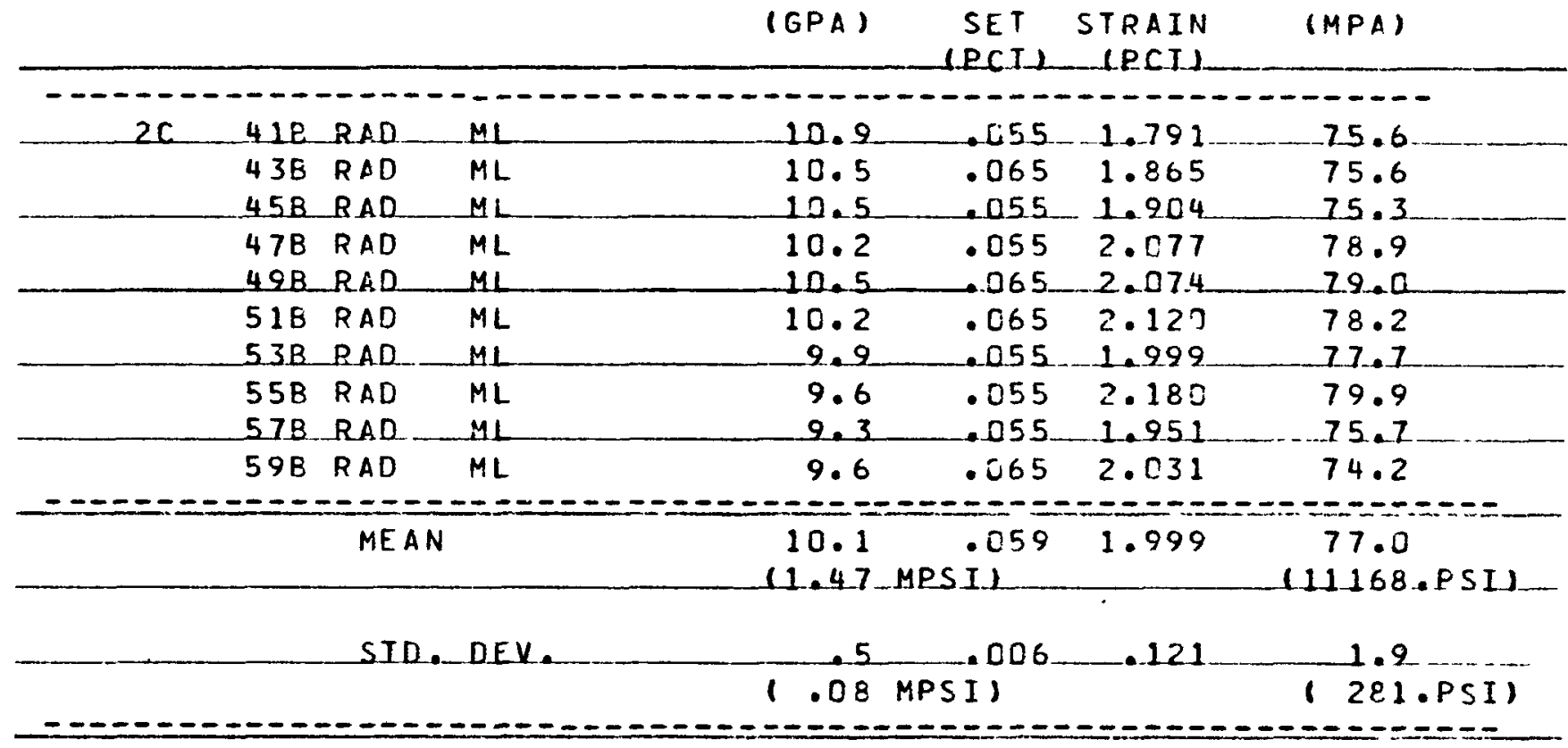


TABLE A-6 (Continued)

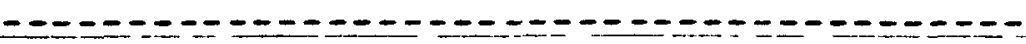

LOT_NO -

SPEC.- DIA.

$12.8 \mathrm{MM}$

LOG NO. $6484 .-137$

SPEC. LENGTH

23. $M M$

LOG DENSITY --

$M G / M+* 3$

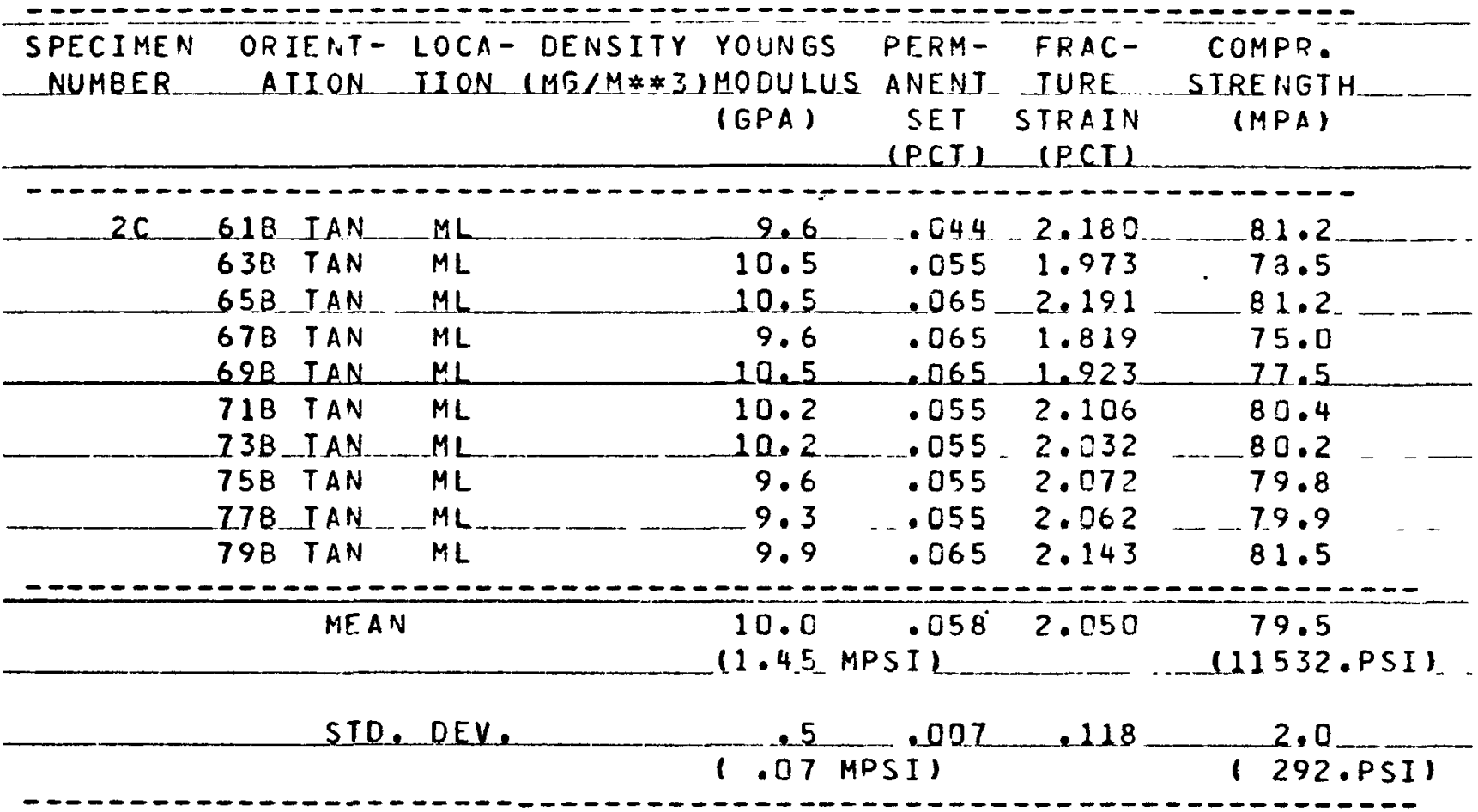


TABLE A-7

COMPRESSIVE PROPERTIES OF GRADE 2020 GRAPHITE (LOG 6799-00)

\begin{tabular}{|c|c|c|c|c|}
\hline $\begin{array}{l}\text { Specimen } \\
\text { No. }\end{array}$ & $\begin{array}{l}\text { Location } \\
\text { in Log }\end{array}$ & $\begin{array}{l}\text { Orientation } \\
\text { of Specimens }\end{array}$ & $\begin{array}{c}\text { Bulk Density } \\
\left(\mathrm{Mg} / \mathrm{m}^{3}\right)\end{array}$ & $\begin{array}{c}\text { Ultimate } \\
\text { Compressive } \\
\text { Strength } \\
\text { (MPa) }\end{array}$ \\
\hline $\begin{array}{l}202 \\
210 \\
218 \\
220 \\
222 \\
228 \\
234 \\
240\end{array}$ & End slab 1 & $\left.\right|_{1} ^{\text {Axial }}$ & $\begin{array}{l}1.780 \\
1.780 \\
1.782 \\
1.783 \\
1.791 \\
1.782 \\
1.781 \\
1.779 \\
\end{array}$ & $\begin{array}{l}79.7 \\
84.8 \\
85.5 \\
84.1 \\
88.2 \\
82.7 \\
89.8 \\
82.0 \\
\end{array}$ \\
\hline $\begin{array}{l}\text { Mean } \\
\text { Std. dev. }\end{array}$ & & & $\begin{array}{l}1.782 \\
0.004\end{array}$ & $\begin{array}{r}84.0 \\
2.5\end{array}$ \\
\hline $\begin{array}{r}3 \\
6 \\
11 \\
14 \\
23 \\
26 \\
31 \\
34\end{array}$ & End slab 1 & $\left.\right|^{\text {Radial }}$ & $\begin{array}{l}1.774 \\
1.765 \\
1.772 \\
1.767 \\
1.782 \\
1.776 \\
1.779 \\
1.776 \\
\end{array}$ & $\begin{array}{l}83.4 \\
80.7 \\
83.4 \\
80.0 \\
84.1 \\
83.4 \\
82.7 \\
77.9 \\
\end{array}$ \\
\hline $\begin{array}{l}\text { Mean } \\
\text { Std. dev. }\end{array}$ & & & $\begin{array}{l}1.773 \\
0.006 \\
\end{array}$ & $\begin{array}{r}82.0 \\
2.2 \\
\end{array}$ \\
\hline $\begin{array}{l}246 \\
256 \\
260 \\
262 \\
265 \\
272 \\
278 \\
280\end{array}$ & $\mid$ & $\left.\right|^{\text {Axial }}$ & $\begin{array}{l}1.779 \\
1.778 \\
1.782 \\
1.781 \\
1.781 \\
1.784 \\
1.776 \\
1.779 \\
\end{array}$ & $\begin{array}{l}80.7 \\
80.7 \\
79.3 \\
79.3 \\
79.3 \\
80.7 \\
77.2 \\
82.7 \\
\end{array}$ \\
\hline $\begin{array}{l}\text { Mean } \\
\text { Std. dev. }\end{array}$ & & & $\begin{array}{l}1.780 \\
0.003\end{array}$ & $\begin{array}{r}80.0 \\
1.6\end{array}$ \\
\hline $\begin{array}{l}35 \\
38 \\
43 \\
46 \\
55 \\
58 \\
63 \\
66\end{array}$ & $\begin{array}{l}\text { Middle slab } 3 \\
\mid\end{array}$ & $\left.\right|^{\text {Radial }}$ & $\begin{array}{l}1.767 \\
1.765 \\
1.770 \\
1.768 \\
1.772 \\
1.766 \\
1.778 \\
1.770 \\
\end{array}$ & $\begin{array}{l}81.4 \\
78.6 \\
83.4 \\
84.8 \\
80.7 \\
82.7 \\
76.5 \\
81.3 \\
\end{array}$ \\
\hline $\begin{array}{l}\text { Mean } \\
\text { Std. dev. }\end{array}$ & & & $\begin{array}{l}1.769 \\
0.004\end{array}$ & $\begin{array}{r}21.2 \\
2.7\end{array}$ \\
\hline
\end{tabular}


TABLE A-7 (Continued)

\begin{tabular}{|c|c|c|c|c|}
\hline $\begin{array}{l}\text { Specimen } \\
\text { No. }\end{array}$ & $\begin{array}{l}\text { Location } \\
\text { in Log }\end{array}$ & $\begin{array}{l}\text { Orientation } \\
\text { of Specimens }\end{array}$ & $\begin{array}{l}\text { Bulk Density } \\
\left(\mathrm{Mg} / \mathrm{m}^{3}\right)\end{array}$ & $\begin{array}{c}\text { Ultimate } \\
\text { Compressive } \\
\text { Strength } \\
\text { (NPa) }\end{array}$ \\
\hline $\begin{array}{l}290 \\
292 \\
298 \\
300 \\
302 \\
318 \\
322 \\
326\end{array}$ & End slab 5 & $\left.\right|^{\text {Axial }}$ & $\begin{array}{l}1.784 \\
1.779 \\
1.784 \\
1.787 \\
1.787 \\
1.786 \\
1.786 \\
1.785 \\
\end{array}$ & $\begin{array}{l}83.4 \\
81.0 \\
85.5 \\
88.2 \\
84.8 \\
89.6 \\
88.2 \\
82.7 \\
\end{array}$ \\
\hline $\begin{array}{l}\text { Mean } \\
\text { Std. dev. }\end{array}$ & & & $\begin{array}{l}1.784 \\
0.003\end{array}$ & $\begin{array}{r}85.4 \\
3.0\end{array}$ \\
\hline $\begin{array}{l}71 \\
74 \\
79 \\
82 \\
87 \\
90 \\
95 \\
98\end{array}$ & End slab 5 & $\mid$ & $\begin{array}{l}1.790 \\
1.784 \\
1.790 \\
1.789 \\
1.793 \\
1.790 \\
1.800 \\
1.800 \\
\end{array}$ & $\begin{array}{l}80.0 \\
87.6 \\
93.1 \\
89.6 \\
91.7 \\
88.9 \\
91.7 \\
88.2 \\
\end{array}$ \\
\hline $\begin{array}{l}\text { Mean } \\
\text { Std. dev. }\end{array}$ & & & $\begin{array}{l}1.792 \\
0.006\end{array}$ & $\begin{array}{r}88.9 \\
4.1\end{array}$ \\
\hline
\end{tabular}




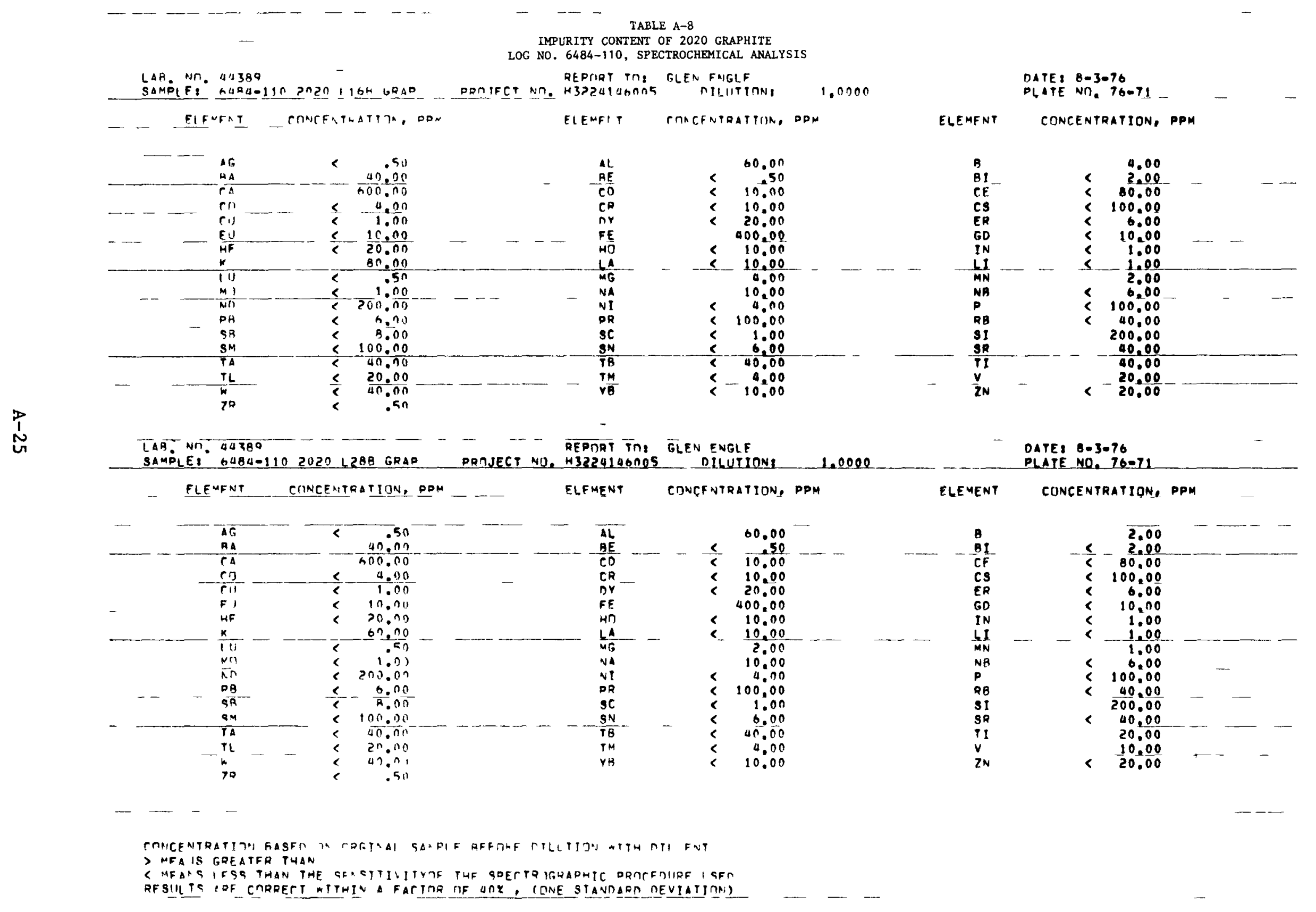




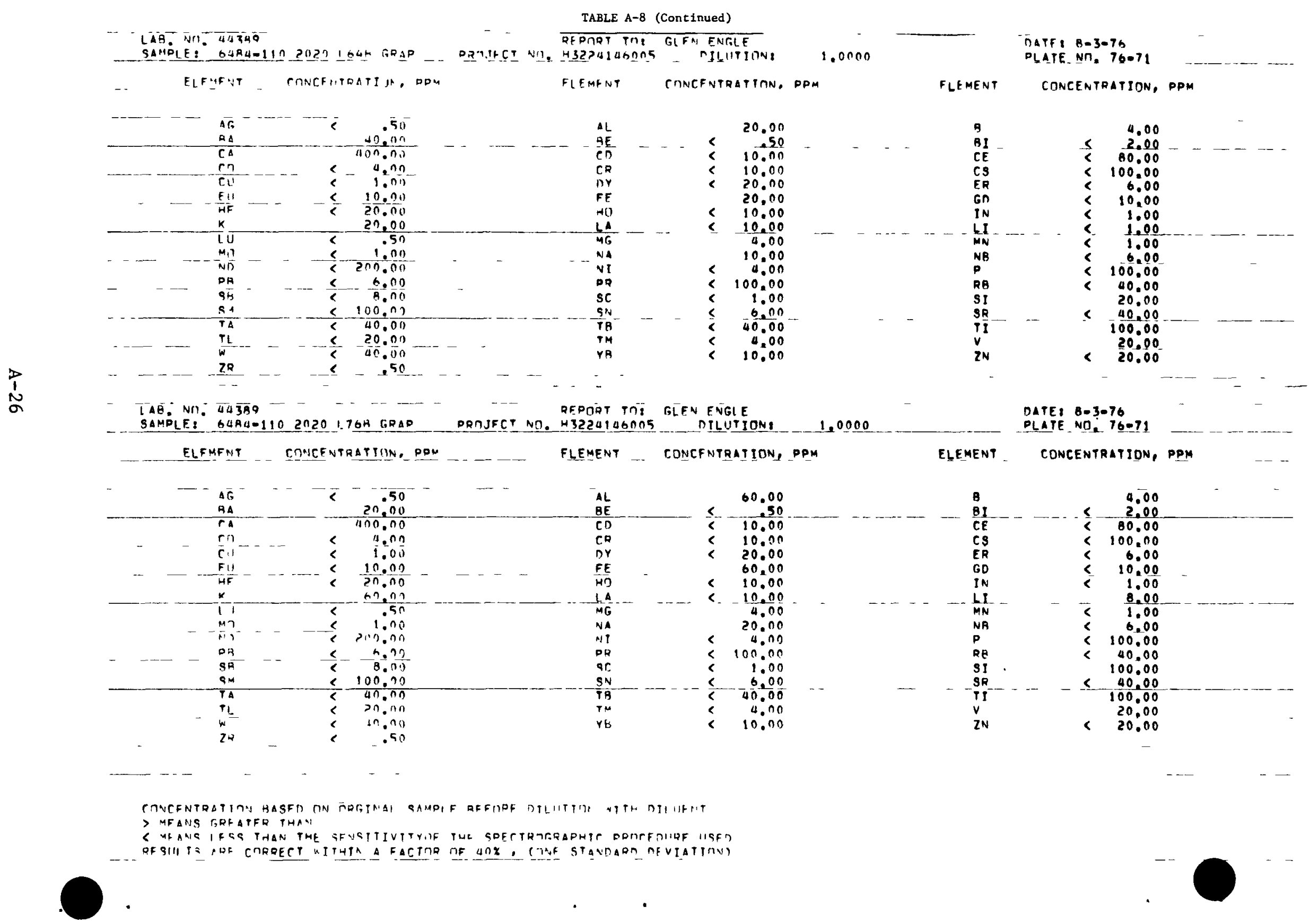




\section{APPENDIX B}

GRADE H-440N DATA

This appendix contains complete data sets for the bulk density, tensile properties, flexural strength, compressive properties, and chemical impurity content of grade H-440N graphite. 
TABLE B-1

TENSILE STRENGTH AND MODULUS OF ELASTICITY DATA FOR GRADE H-440N

$\begin{array}{lrr}\text { LOT NO. } & \text { SPEC. DIA. } & 12.8 \text { MM } \\ \text { LOG NO: 6484-81 SPEC. LFNGTH TO. MM } \\ \text { LOG OENSITY -- MG/M\$3 }\end{array}$

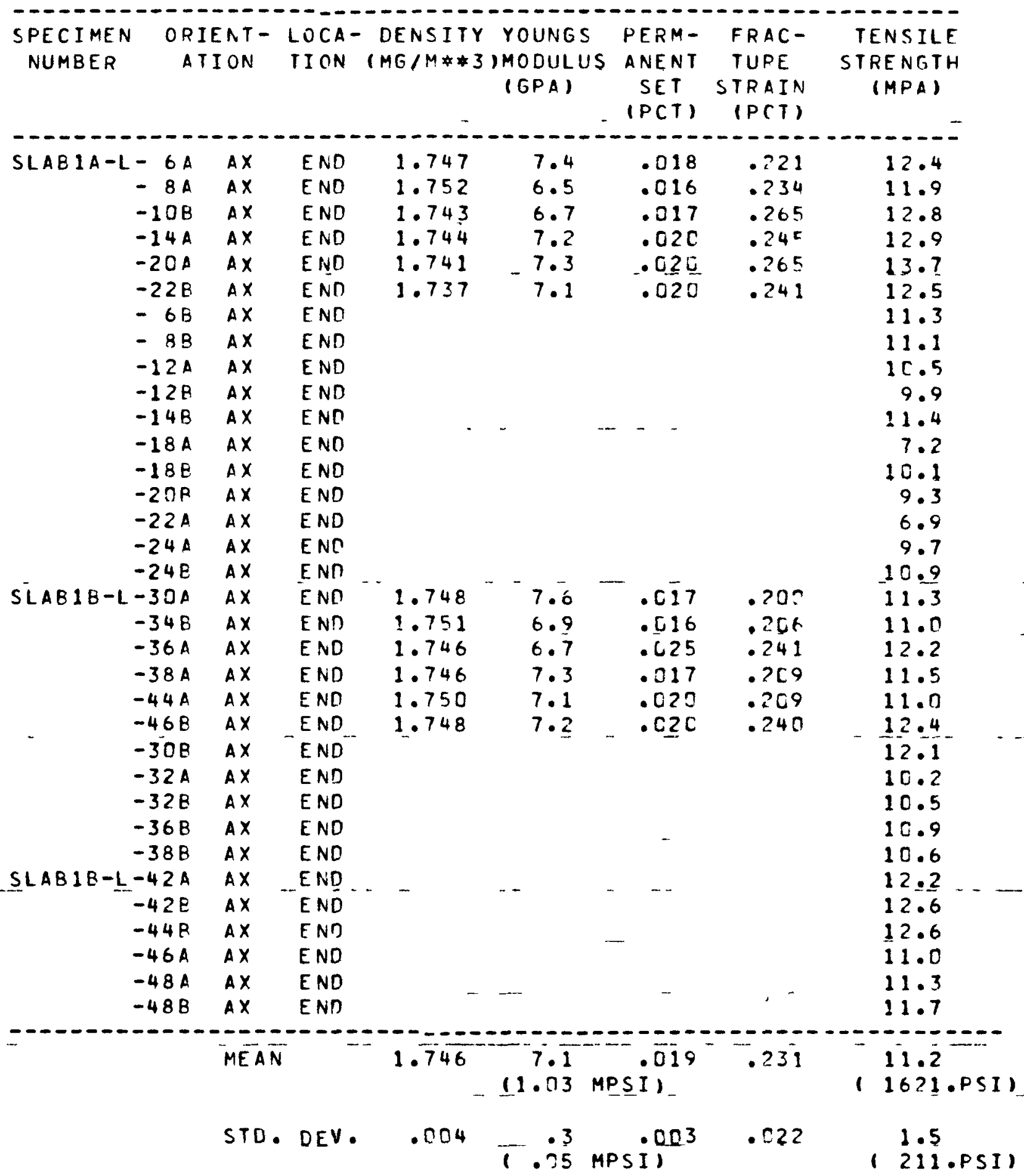


TABLE B-1 (Continued)

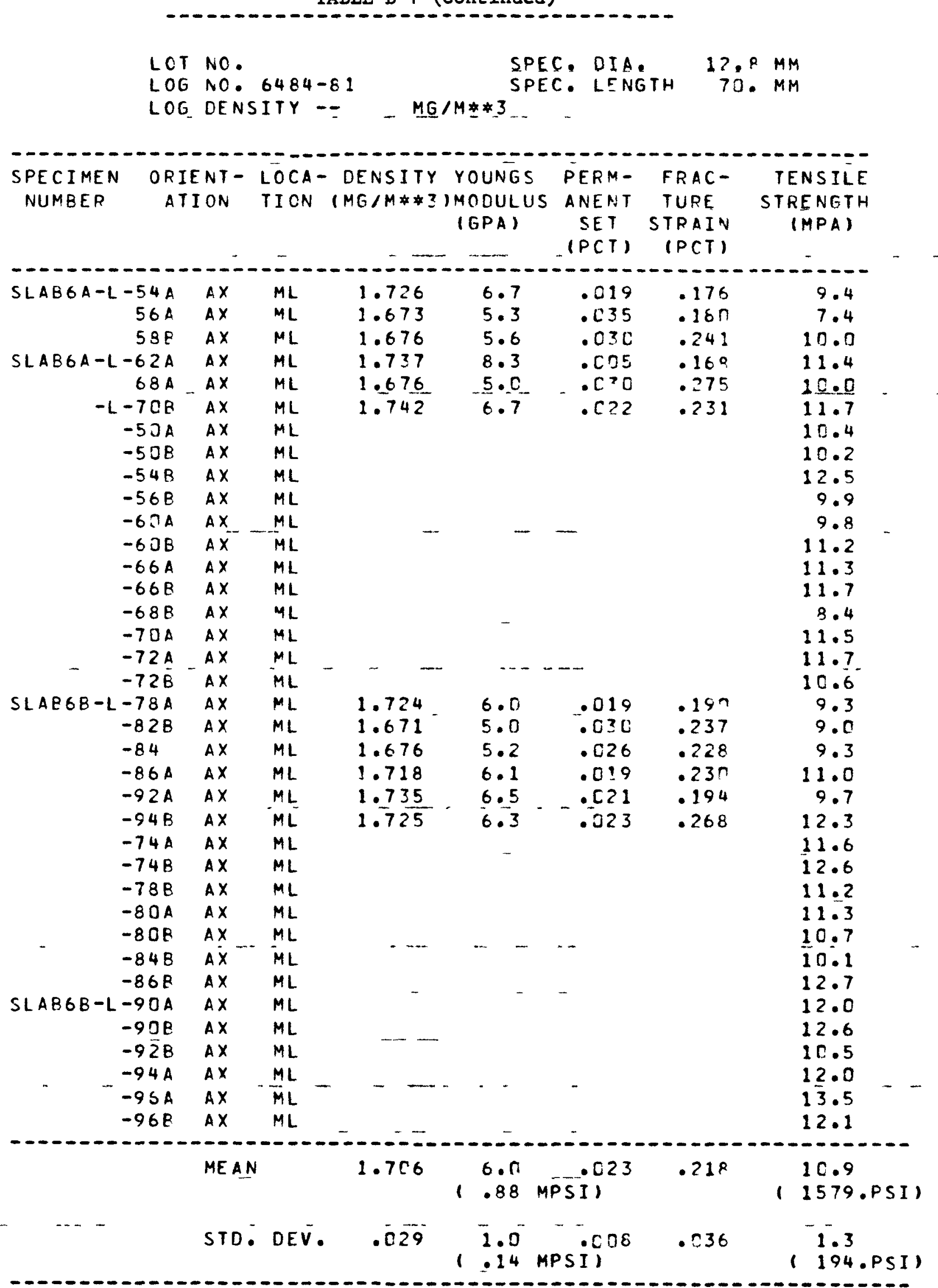


TABLE B-1 (Continued)

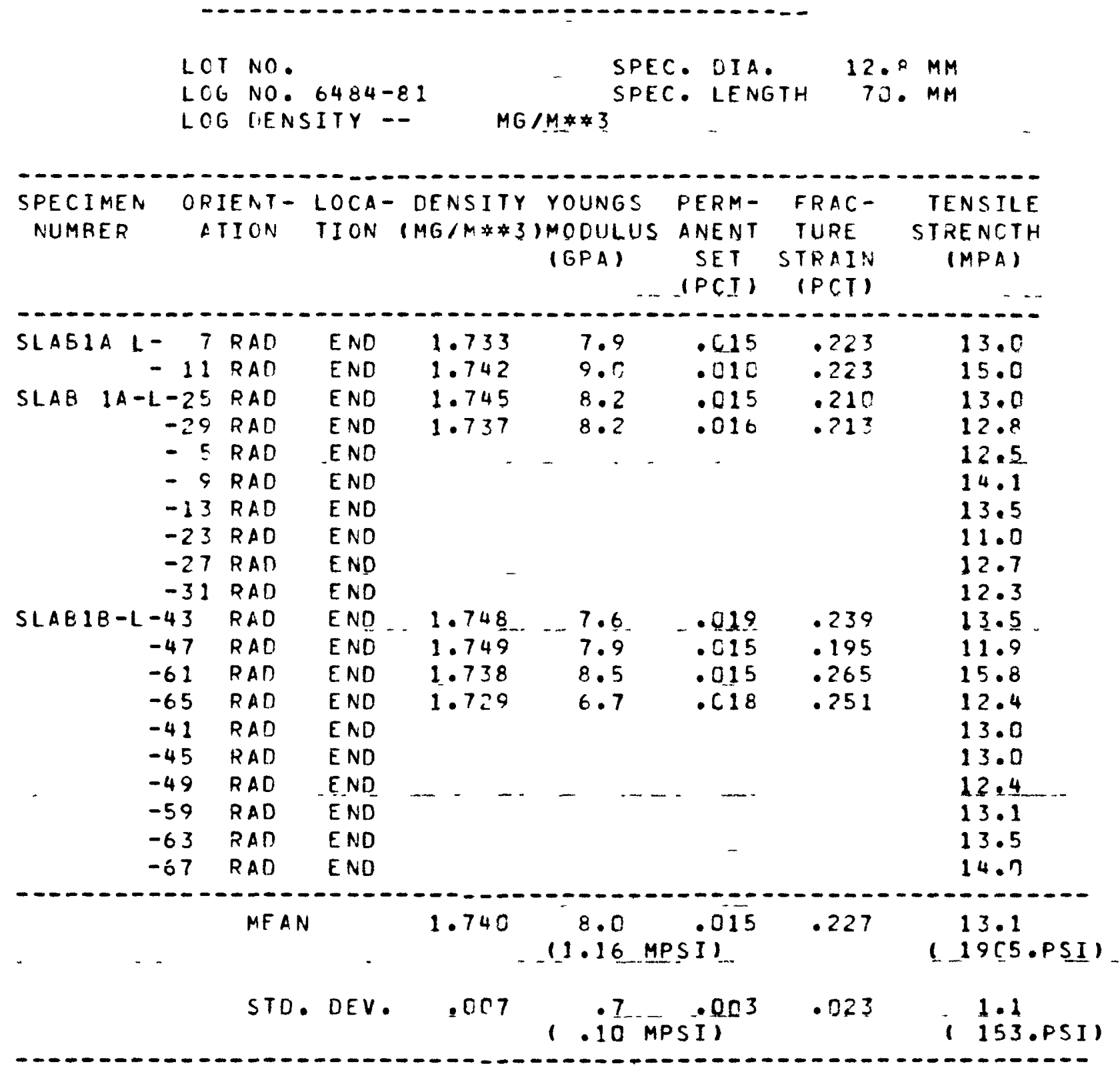


TABLE B-1 (Continued)

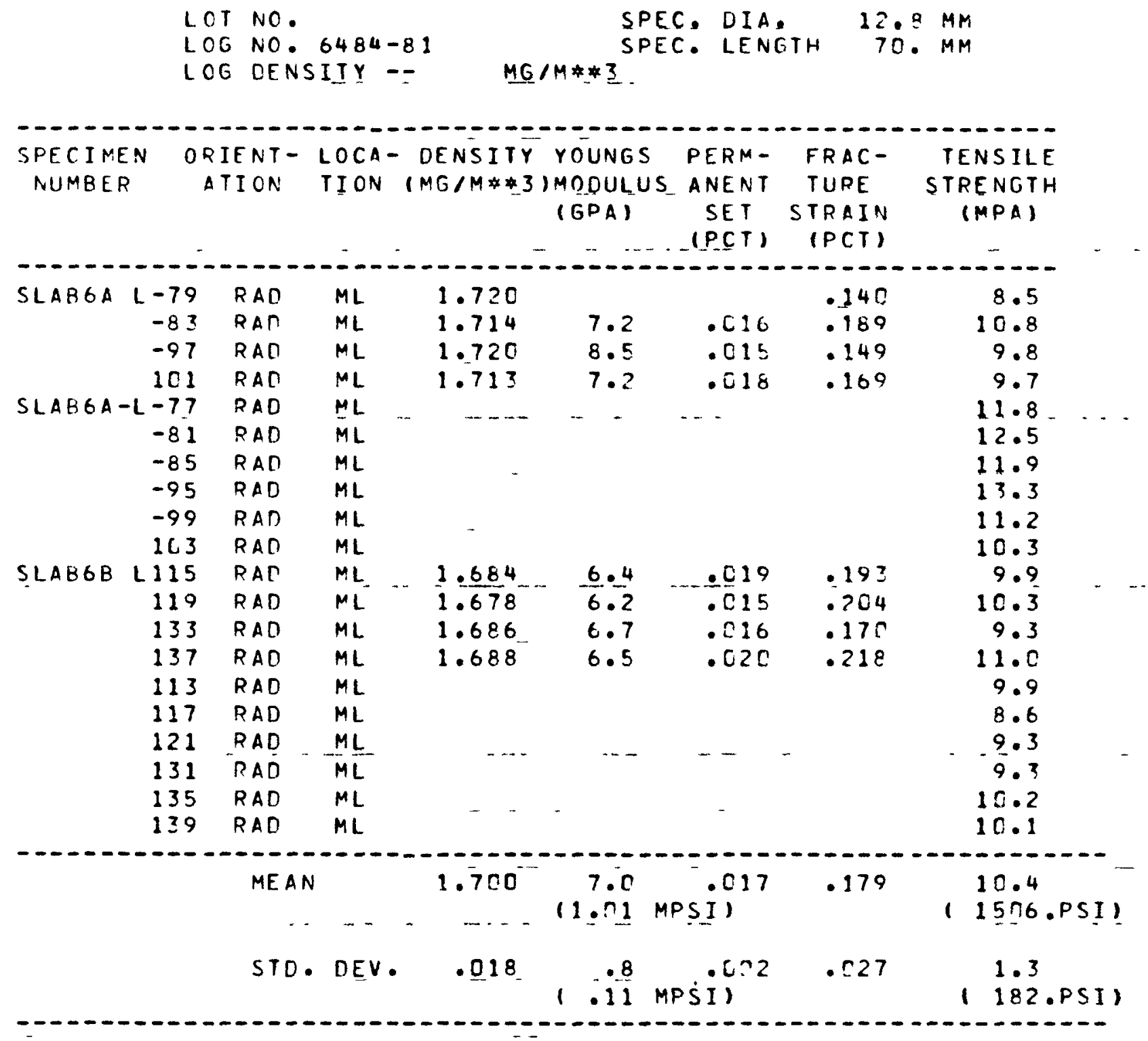


TABLE B-2

FLEXURAL STRENGTH DATA FOR GRADE H-440N

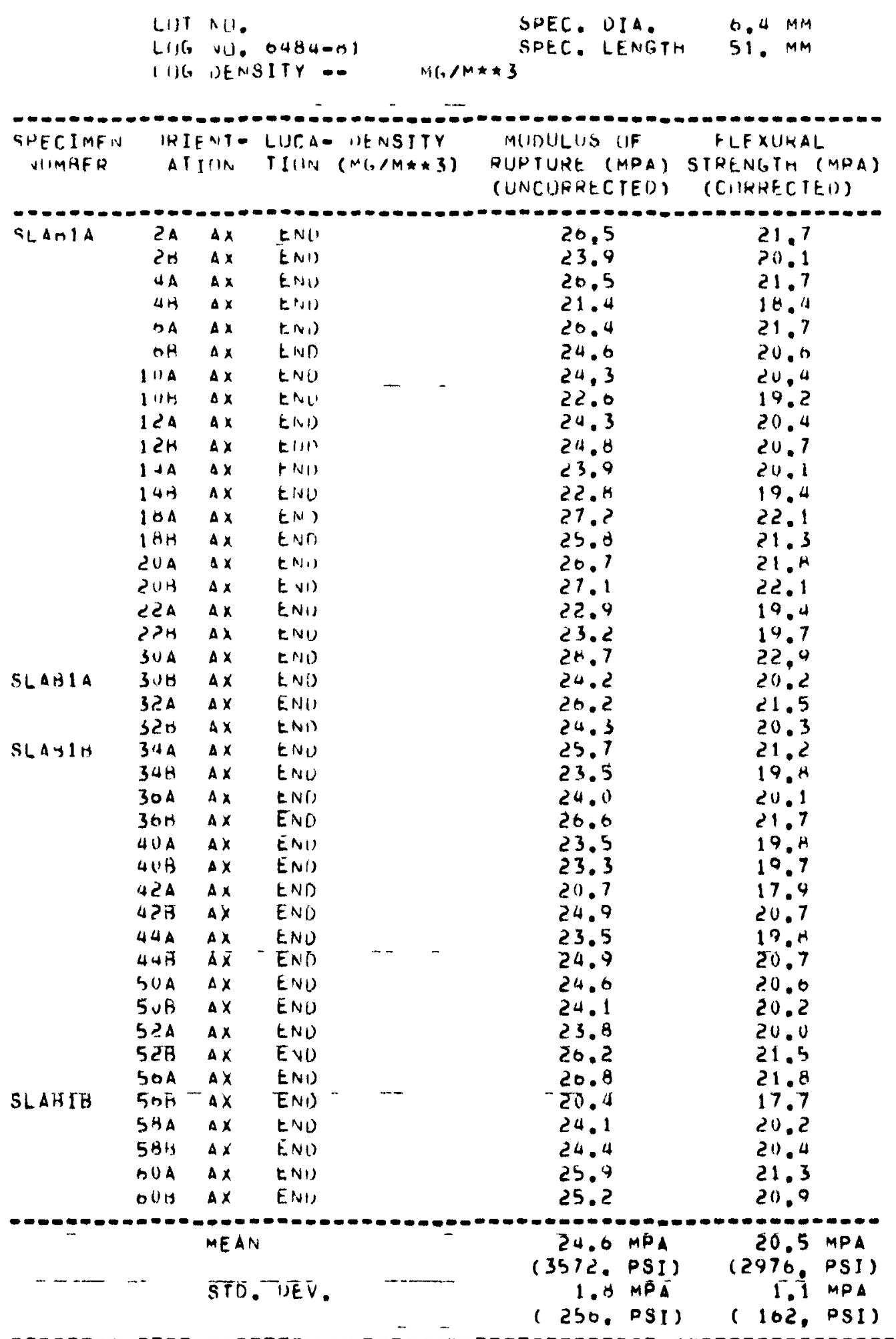

B-6 
TABLE B-2 (Continued)

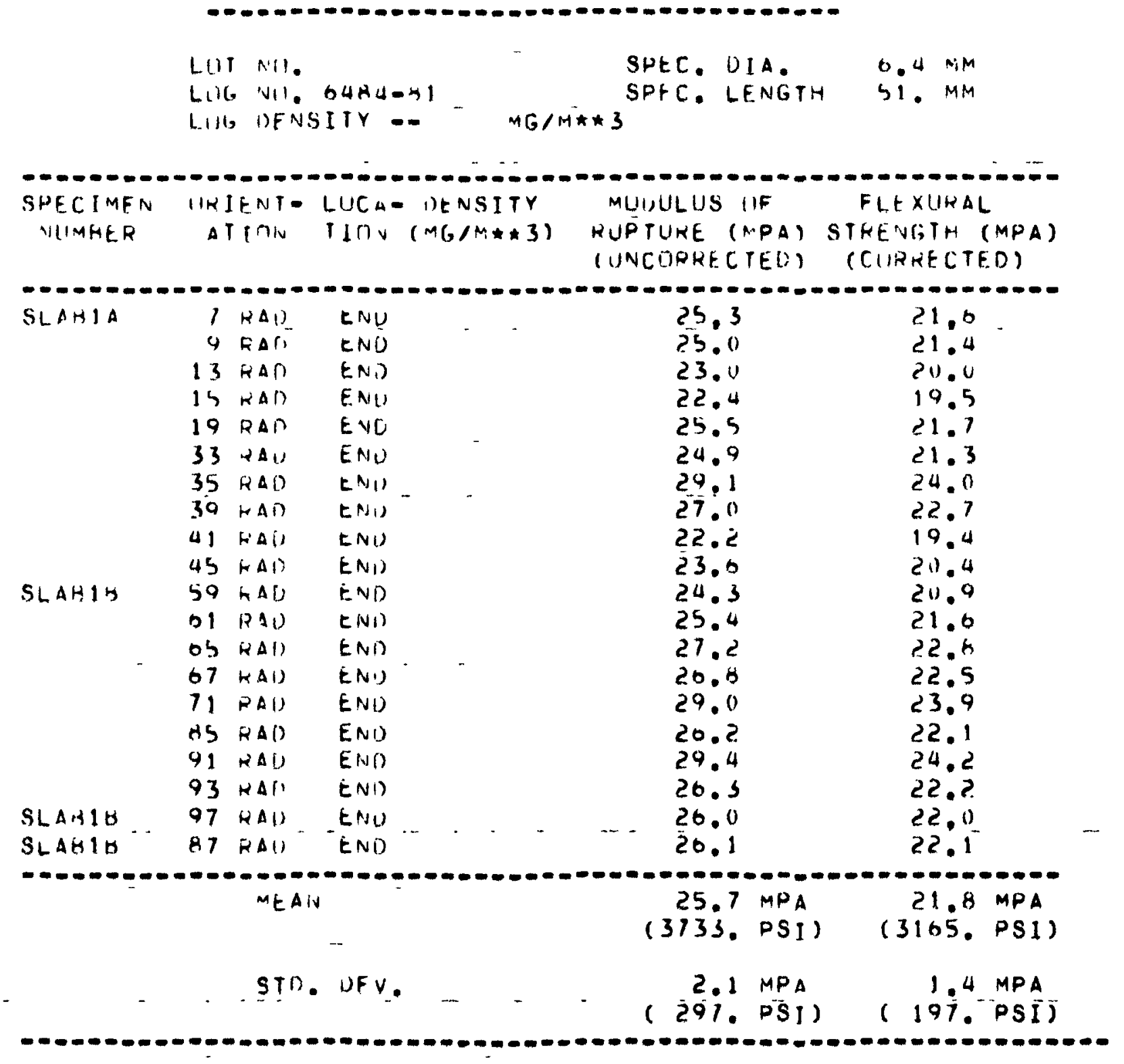


TABLE B-2 (Continued)

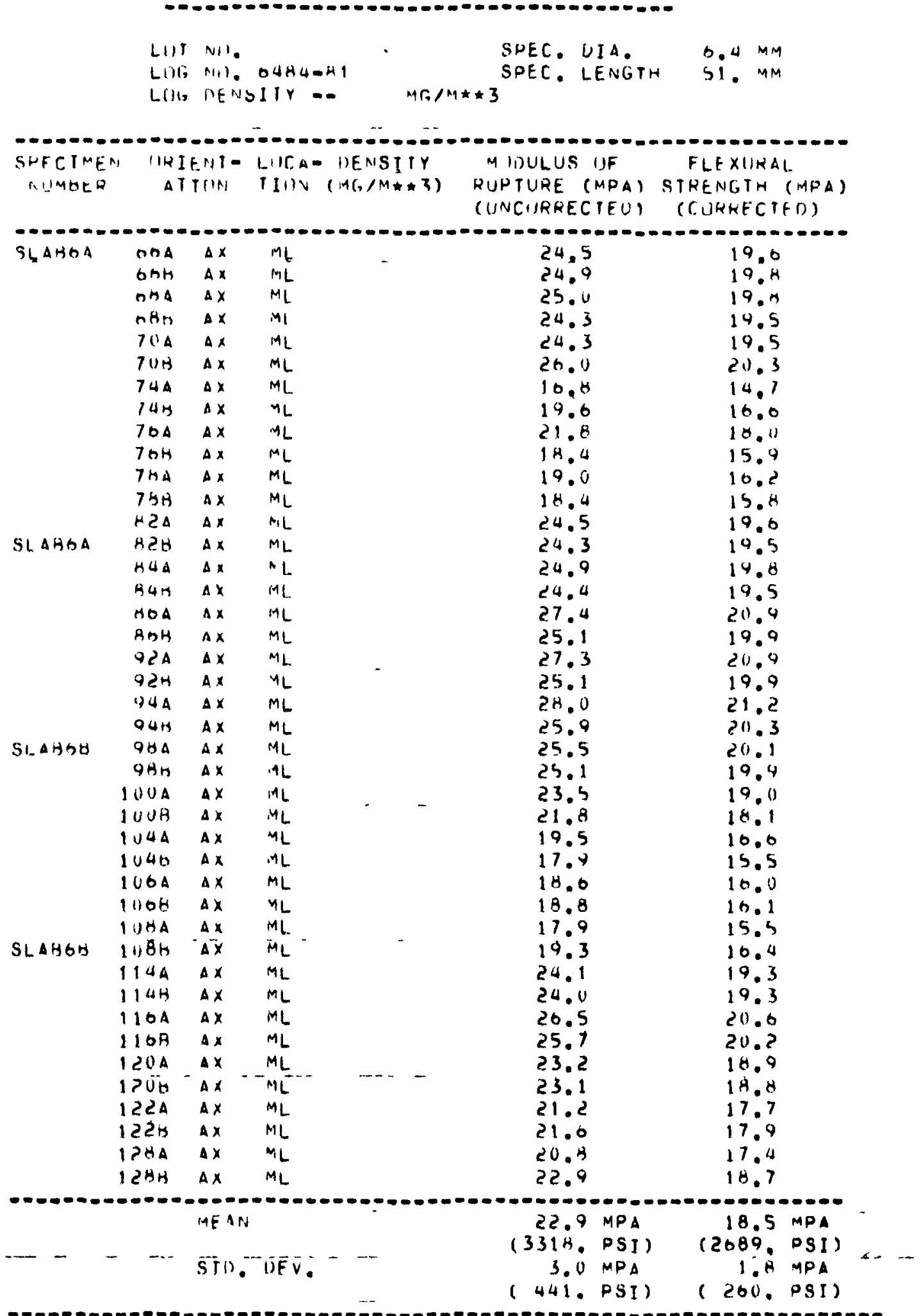


TABLE B-2 (Continued)

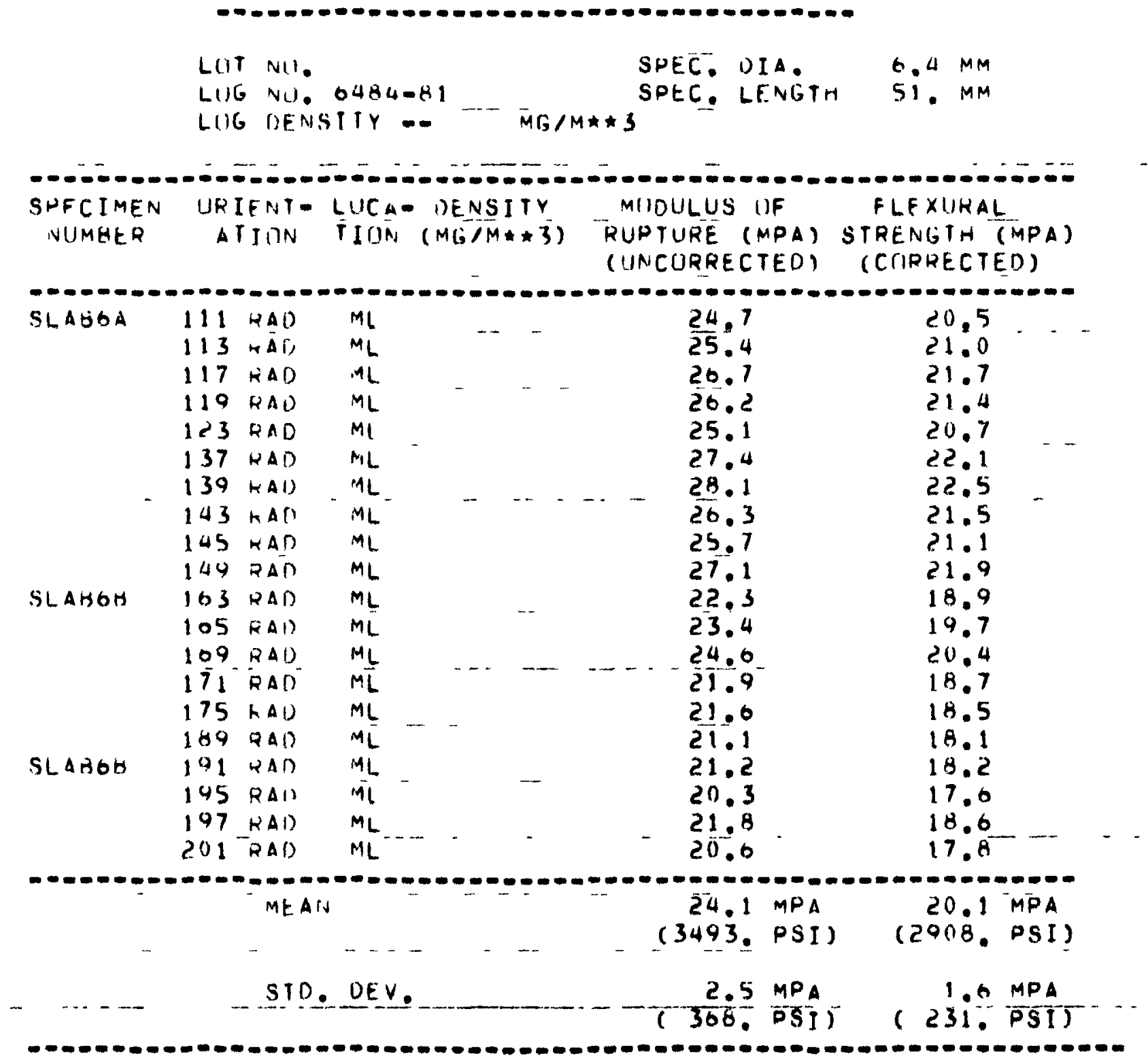


TABLE B-3

COMPRESSIVE STRENGTH AND MODULUS OF ELASTICITY FOR GRADE H-44ON

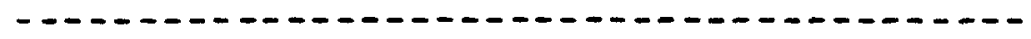
L. T NO.
SPEC. IIA. Z?.'MM
L G NO. 64\&4-81

L. $O$ [ENSITY -

$M G / M *$ ?

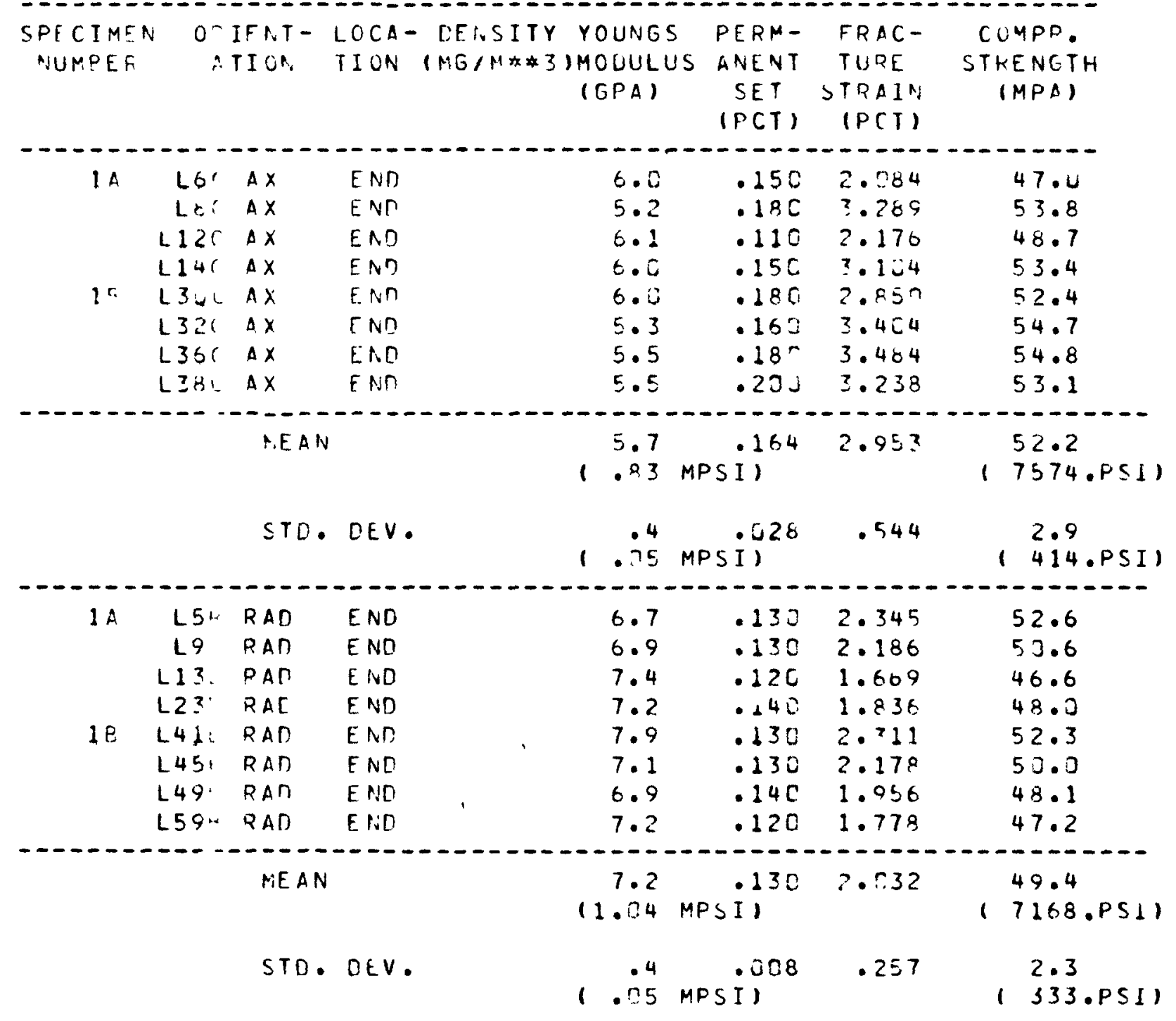


TABLE B-3 (Continued)
LIT iNO.
SPEC. DIA. 12. = MM
LIG NO. $6484-81$
L:C DENSITY --
$M G / M * 3$
SPEC. LEPGTH ?2. MM

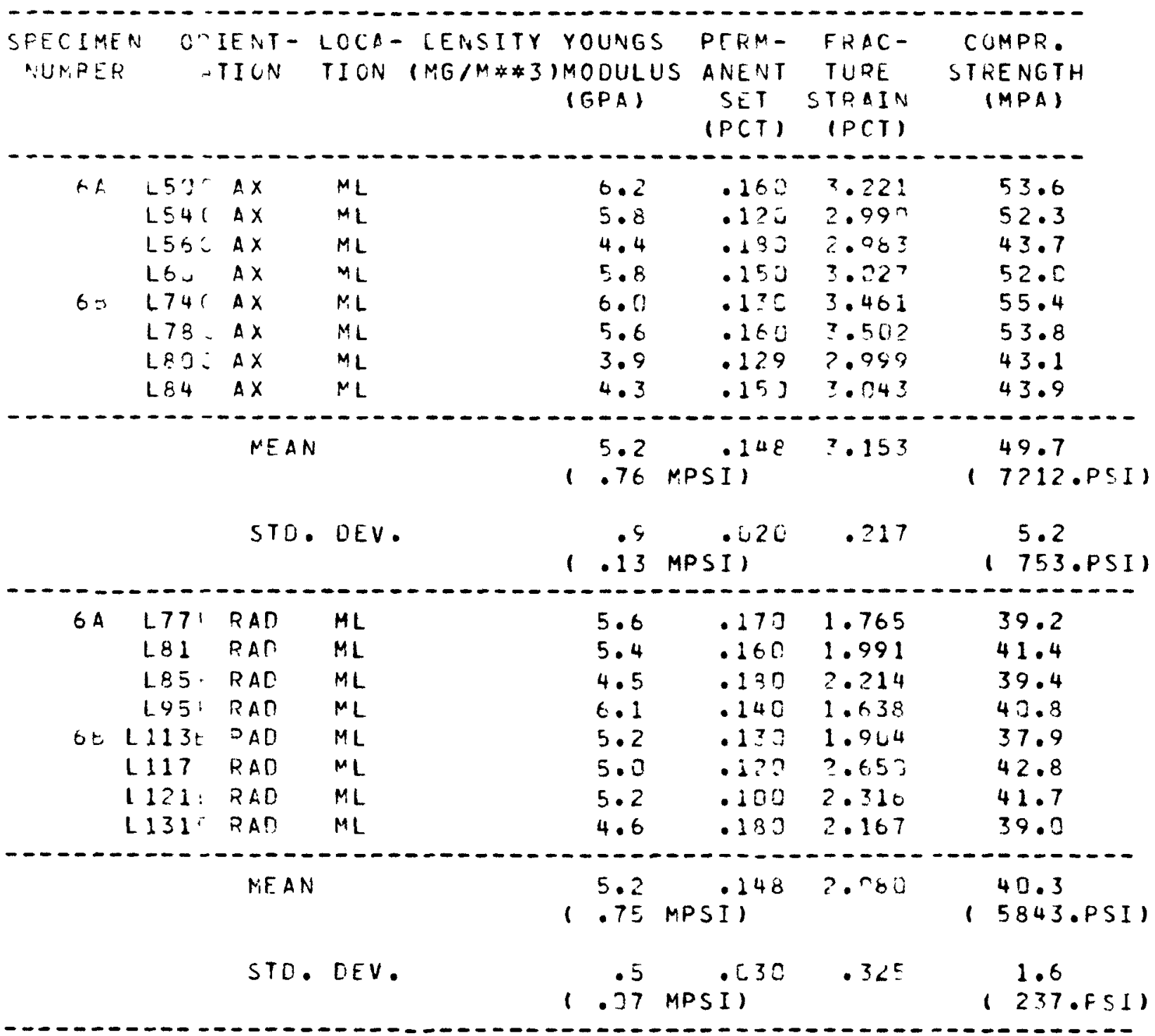




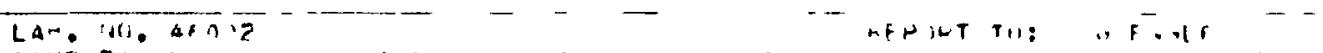

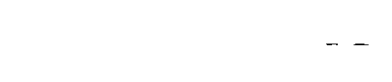

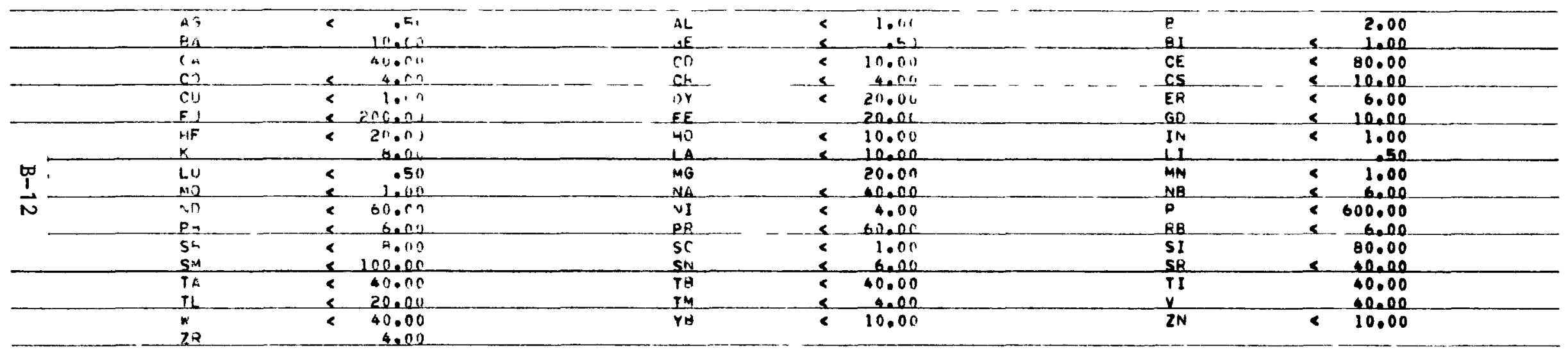

COLCF TRAT IOF 3ASEO UN ORG INGL SAMPLE BEFORE OILUTION $*$ ITH DILUENT

C MEAUS GPEATER THAN

RESULTS APE CORRECT WITHIT A FACTOE UF QU\%, COME SIANDARD DEVIAIJON 


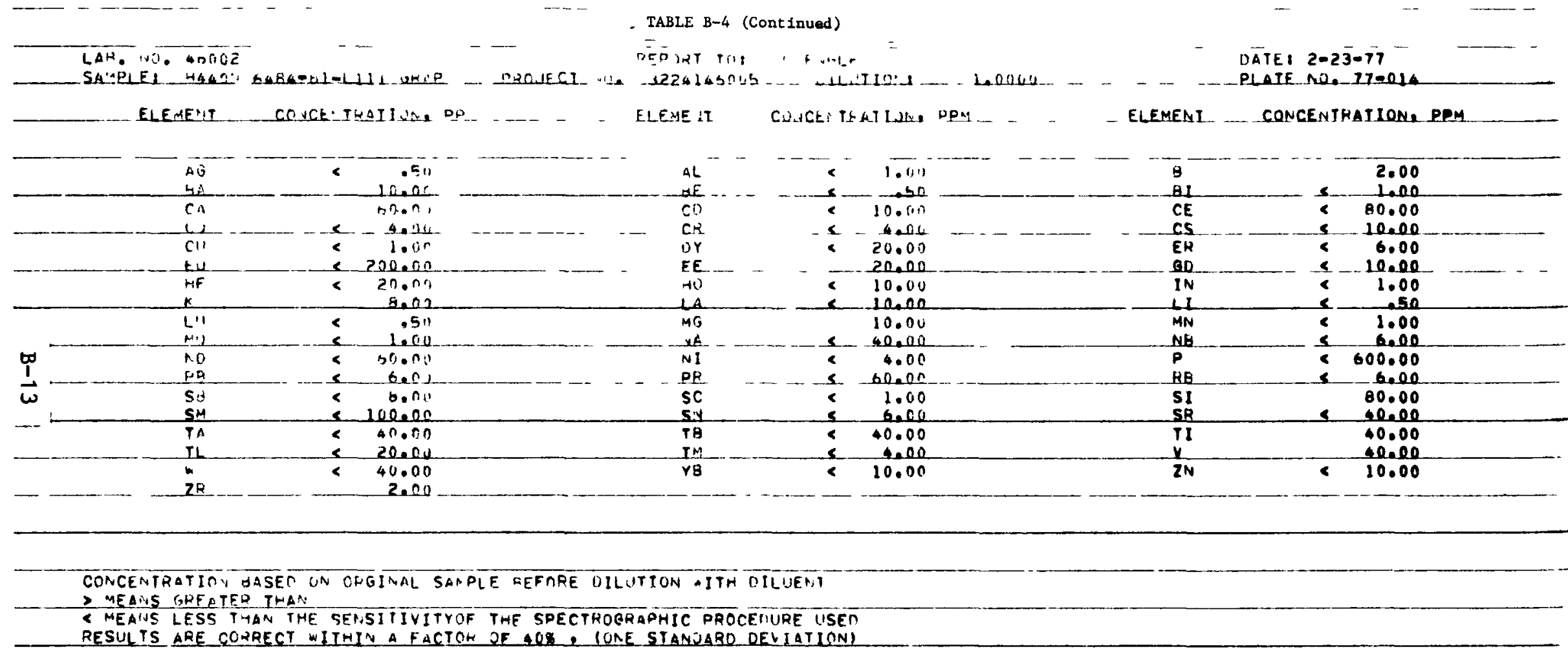

TABLE B-4 (Continued)
DATEI $2-2 \overline{3}-7 \overline{7}$

CONCENTRATIONE PPM 
TABLE B-4 (ContInued)

LAE. NC. 61112 ? $-\cdots$ ELEMENT COHCENTEATLL, DP. ELEIEIT CONCE:THALUOR. PPM

CONCENTHATION BASED OII CRGI'TAL SAMPLE TEFOFE UILUTION WITH OILUENT

- MEANS GREATER THAM

- MEANS LESS THAN TAF SENSITIVITYOF THE SPECTROGRAPHIC PAOCEDURE USED

RESULTS AAE CORAECT WITHIN A EACTOP OF AOK, CONE STAMDARD DEYIAUIOMU 
TABLE B-4 (Continued)

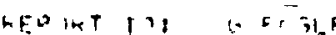

LAB. NG. 40012 2

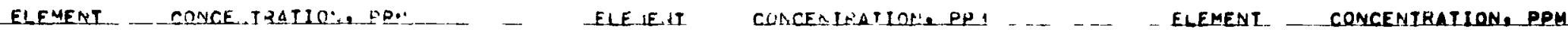

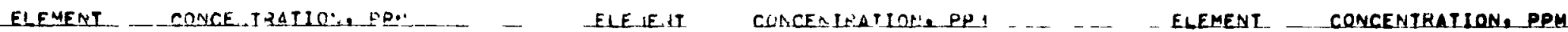

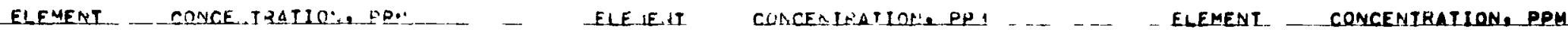

UATE: 2-23-77

PLAIE NOE TT-016

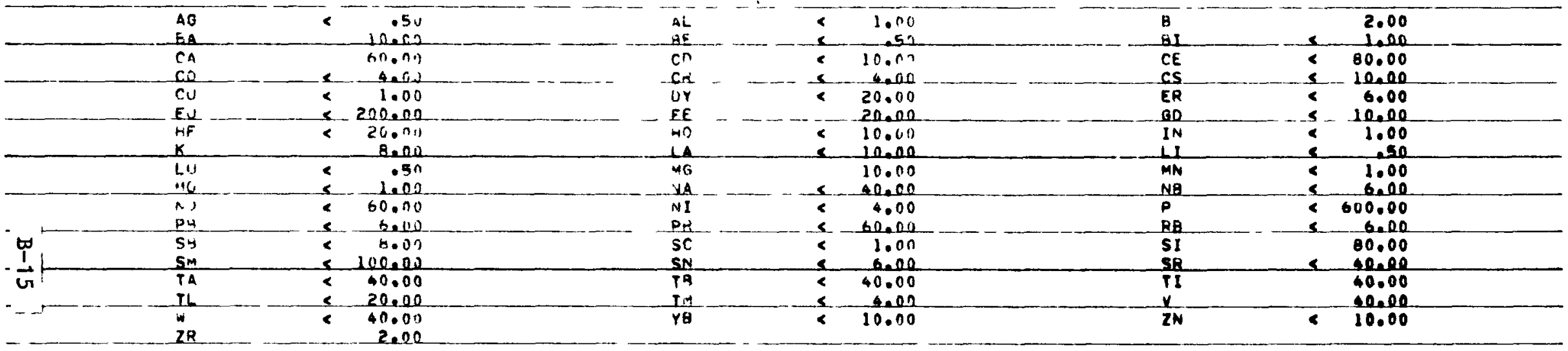

CONCENTRATION 3ASEN ON CQUINAL SAMPLE FEFORE OILUTION WITH OILIJENT

- MEATS GREATER THAN

RE MENS LESS TWA"I THE SENSITIVITYOF THE SPECTROGPAPHIC PROCEDURE USED

RESULTS ARE COARECT WITHIN A FACTOK OE DO LONE SIANDARO DEVIALIONI 


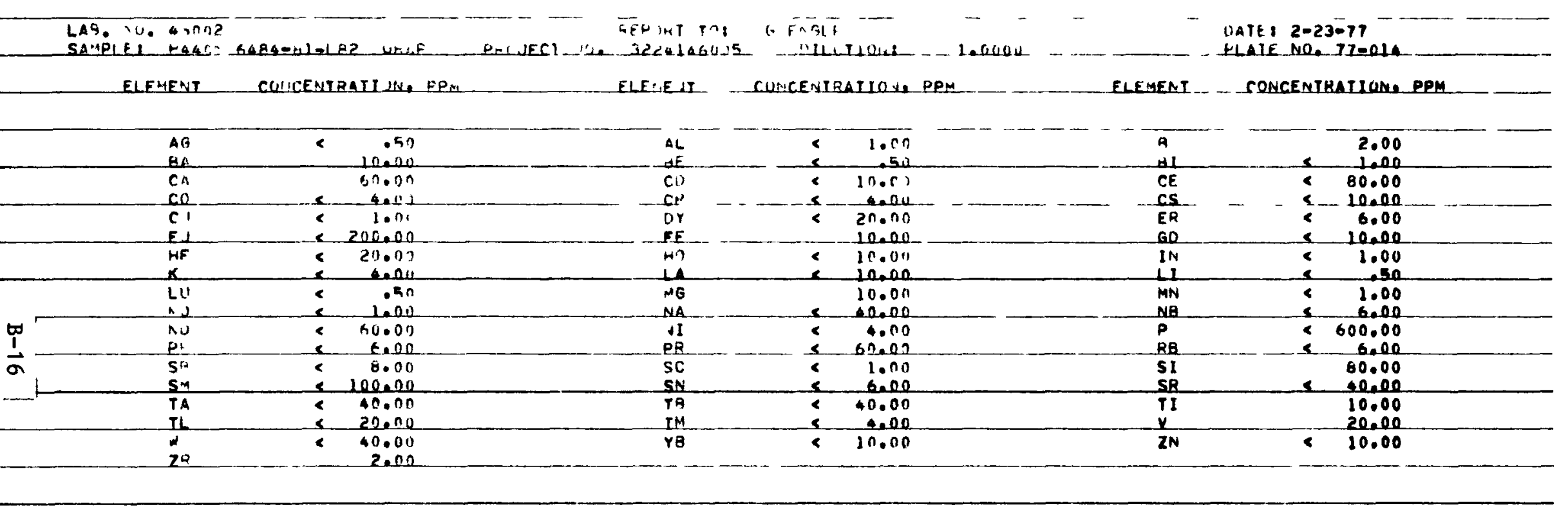

CONCEHTRATIOO OASEN NV ORGINAL SAMPLE FEFOFE OILUTIJU WITM OILUENT

$\rightarrow$ MEALS GREATER THAN

C MEANS LFSS THAN TME SE JSITIVITYOF THE SPECTRGgeapHIC PZOCEOURE USED

PESILTS ARE COQRECT WITHIU A FACTOR OF OQS IONE STANOABO OEVIAIIONI 
LAG. 40. agOn?
UATE, $2-23-77$

- PLAIE NOE TI-0La

ELENENT ... CONCENTKAIIUN, PPM

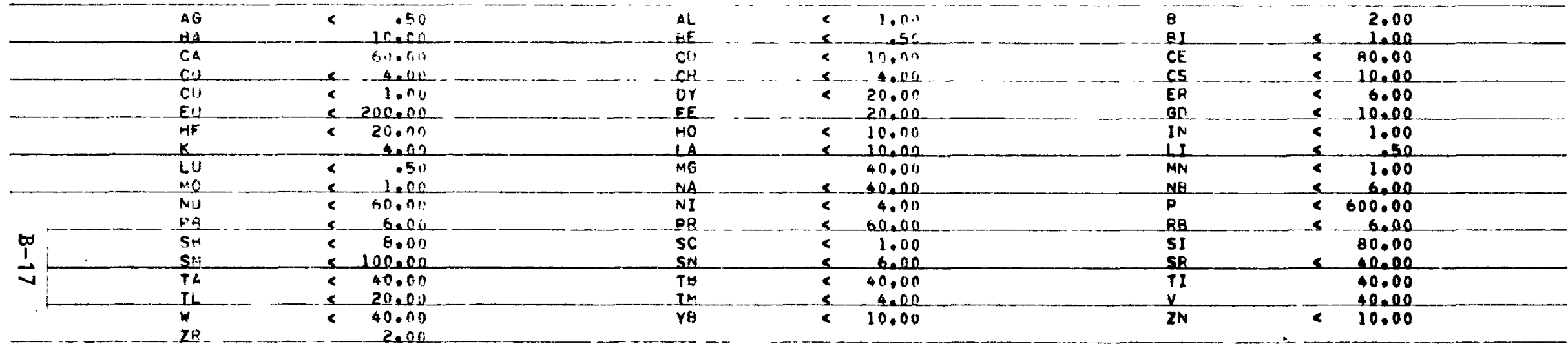

CONCENTRATION BASEU ON ORGINAL SAMPLE REFORE OILUTIOA WITH OILLENT

$\rightarrow$ MEANS GLEATEE THAN

C MEAIVS LESS THLA THE SEISITIVITYOF THE SPECTROGRAPHIC PPOCEUUNE USED

RESULTS ARE CORRECI WITH A FACIOR OF ON LONE STAYOARO OEYIATION) 
TABLE B-4 (Continued)

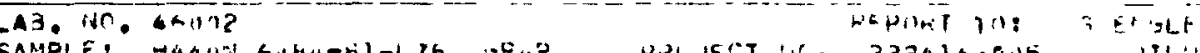

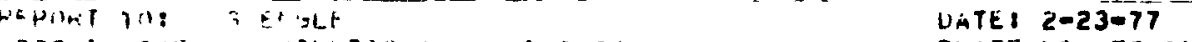

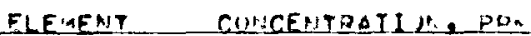

ELEMEVT COICE IFAllOA. PPY

ELEMENT COMGENTRATION PPM

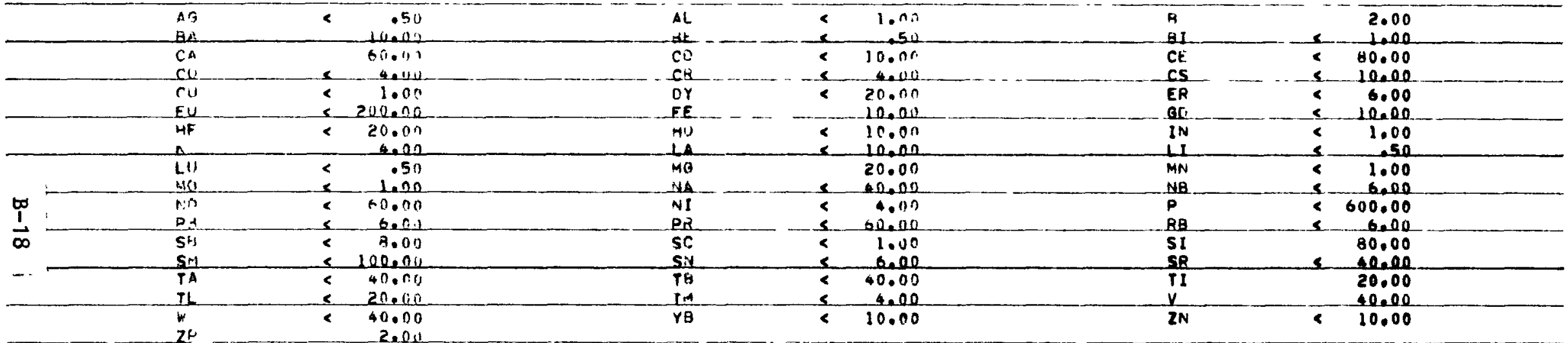

CONCENTRATIOH OASED ON ORGINAL SAMPLE REFORE DILUTION WITH OILUENT

- NEANS GKEATER THAN

- MEANS LESS THANI THE SEISITIUITYOF THF. SPECTROGRAPHIC PROCEUURE ISED

RESULTS ARE COQRECT WITHIN A FACTOK OE OO, CONE STANDARD DEVIUIIONS) 


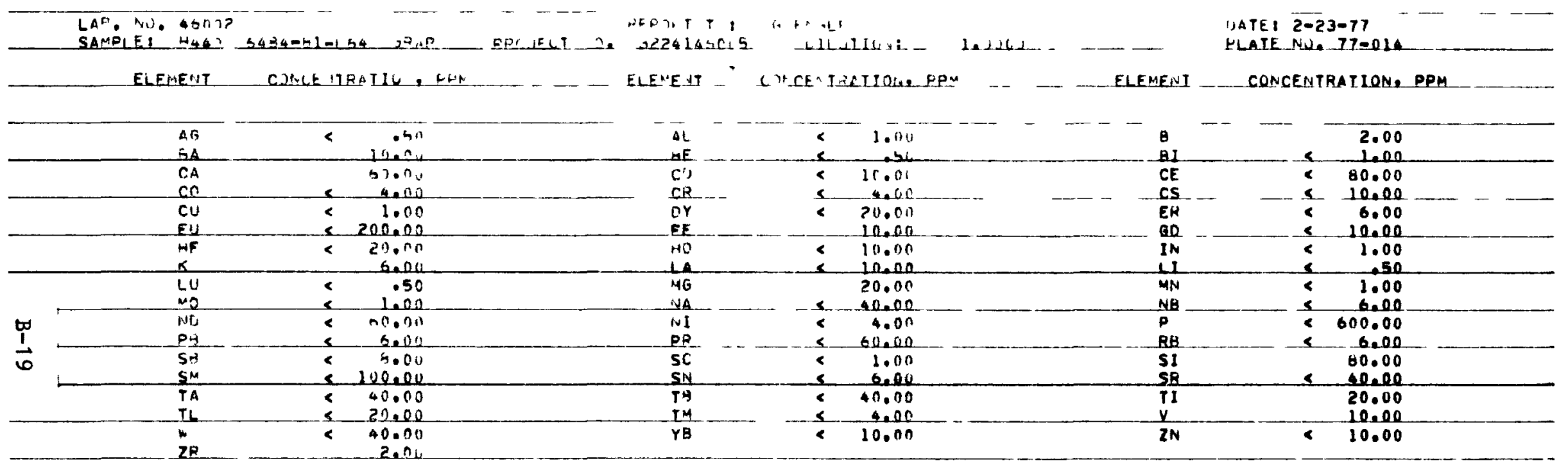

CONCENTRATION GASED ON. ORGINAL SAMPLE REFOHE OILUTIUN WITH DILUENT

? PAATS GREATEH THAIY

C MEANS LESS TAA THE SENSITIVITYOF TWE SPECTROGRAPHIC PPOCEDURE USEU

RESULTS ARE COARECT WITHIN A FACTOR OF OOR, (ONE STAMPARO DEVIATLONI) 
AB. 10.640 .12

00 TEI $2-23-77$

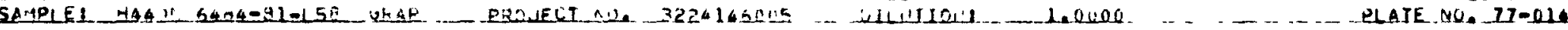

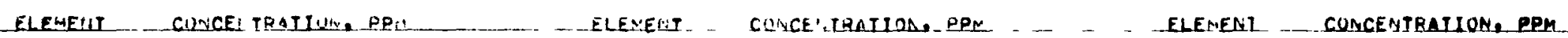

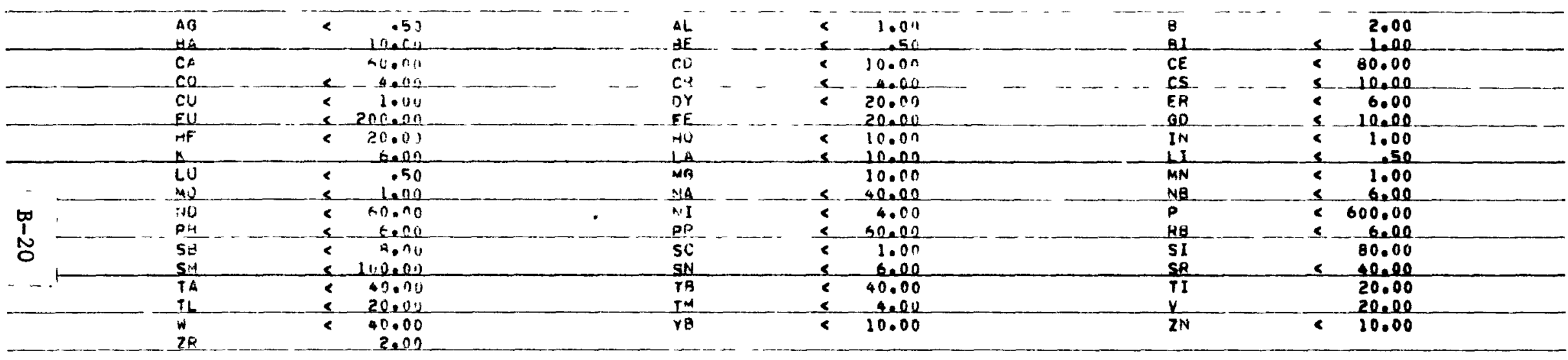

COMCENTRATION BASED O. ORGIIAL SHMPLE BEFONE OILUTIOII NITH OILUEMT

C MEANS GREATEK TMAI4.

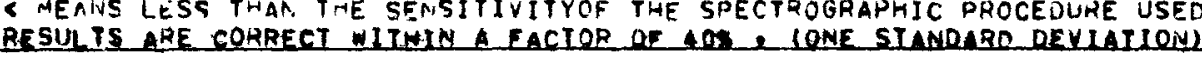


TABLE B-4 (Continued)

LAR. $A G, \overline{4 a n Q ?}$

SAHPLE

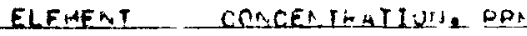

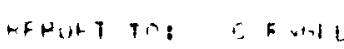

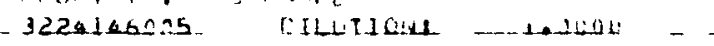

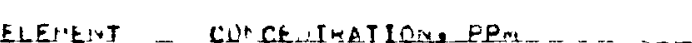

TATE: $2-23=77$

PLAIE TH. TTE0I

ELEMEAI _. CONCENTKAIION. PPM

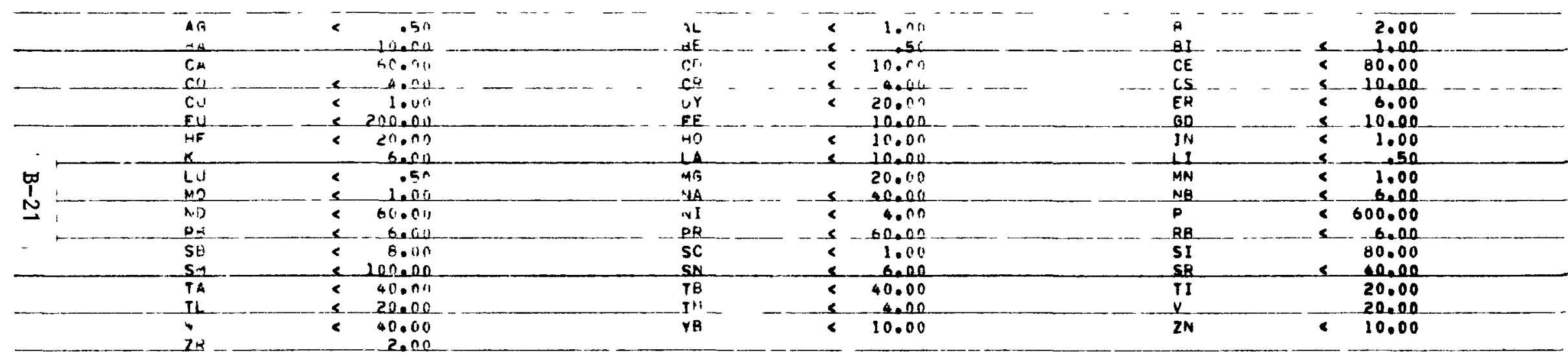

CONCENTRATIOK GASED ON ORGINAL SAMPLE REFORE DILUTIOH WITH OILUENT

C MEATS GPEATER THA:

C MEASSS LESS THAN THE SENSITIVITYUF TME SPECTROGRAPHIC PROCEOURE USEO

RESULTS ARE CORRECI ITHIN A EACTOR OE AOS. IONE SIANDARD DEVIAIIONI 
TABLE B-4 (Continued)

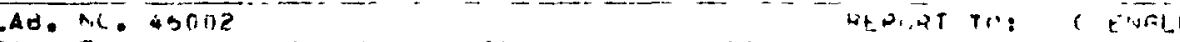

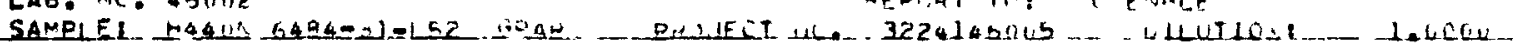

CATEI $2-23=11$

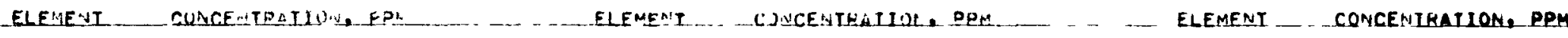

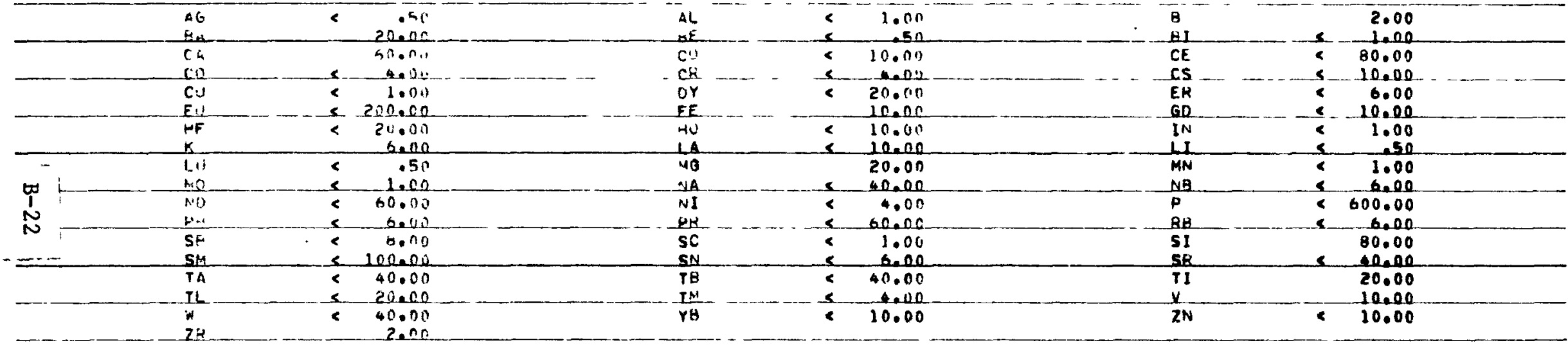

CONCFMTRATION BASED UA TPGIMAL SAMPIE NEF ORE OILUTION WITH DILUENT

MEAHS GPEATER THAM

MEANS LESS THAN THE SFASITIVITYOF THF SPECTROGRAPHIC PROCEDLRE USED

RESILLTS ARE CQRRECT WITHIN A FACTOR OF ROS, (ONE STANDARO DEVIATION) 
TABLE B-4 (Cont inued)

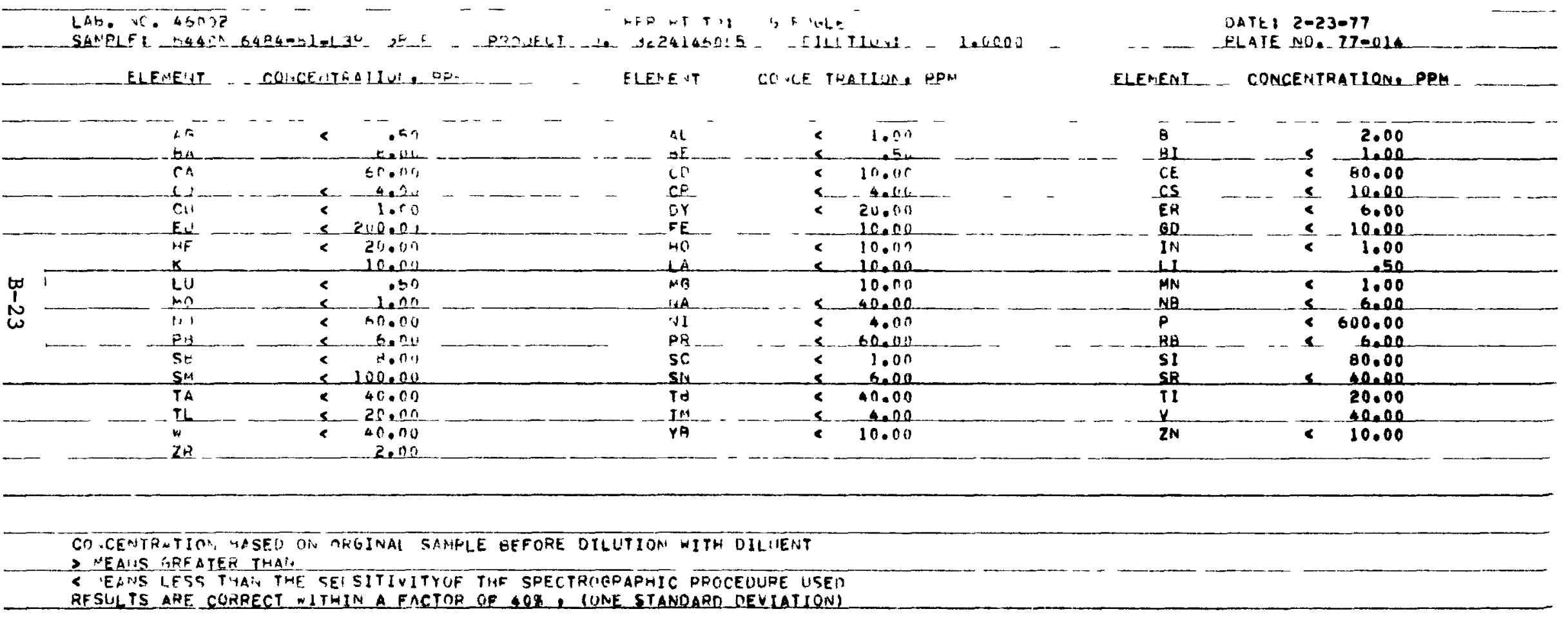

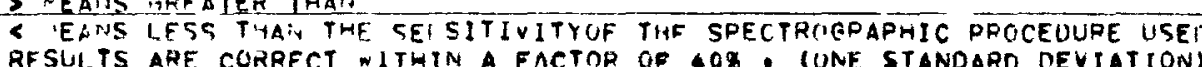

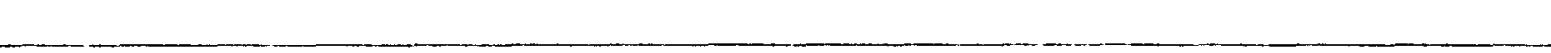

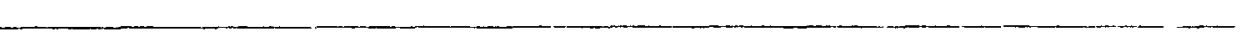




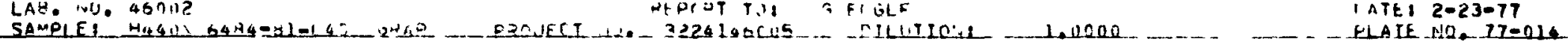

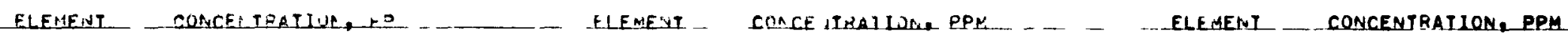

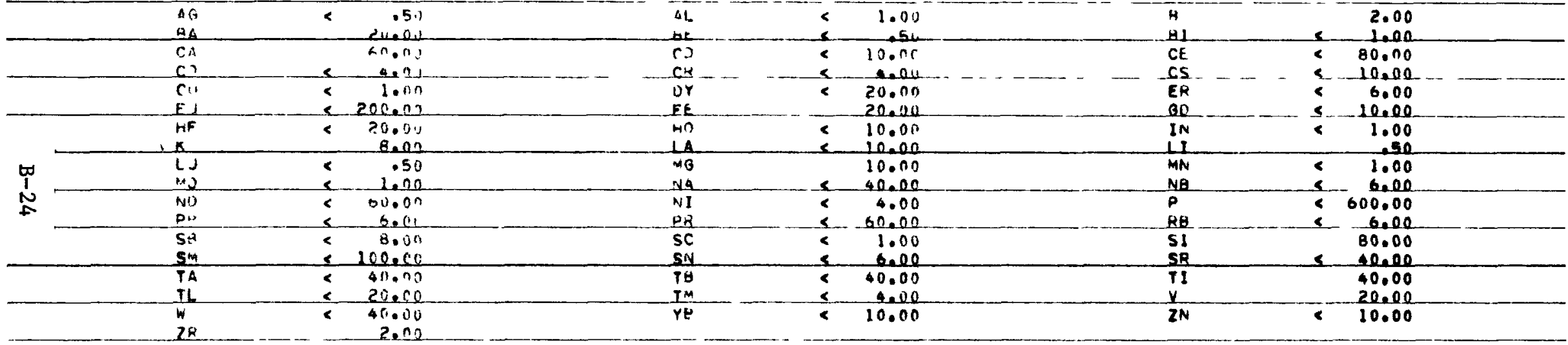

CONCEHTRATION OASED ON ORGIWAL SAMPIE AFFORE OILUTION ITTM DILUETAT

C MEALS LESS THAR. THE SENSITIVITYOF THE SPECTROGKAPAIC PAOCENLRE USEO

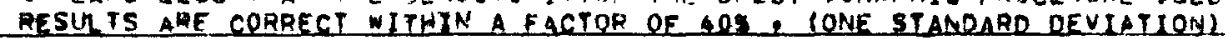




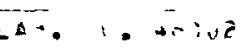

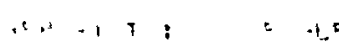

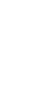

Sc. iltei +48

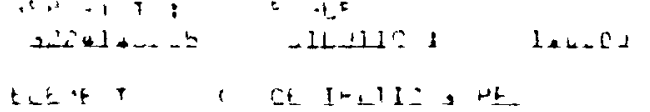

$-\cdots$

InTE: ?-23-77

ELEET OCERHA-

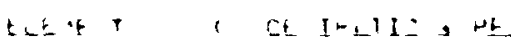

ELE: EY)

AIE Ti. 77-414
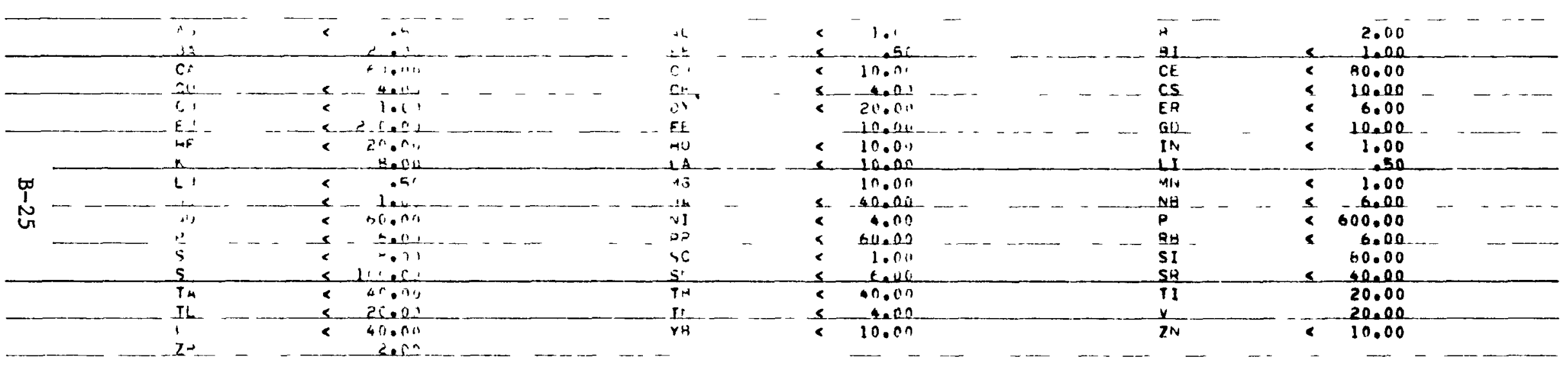

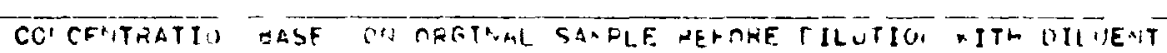

$\rightarrow$ FA'S GMEATER I'UA

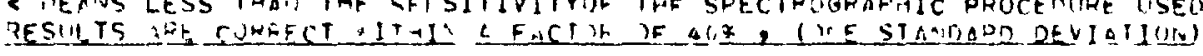

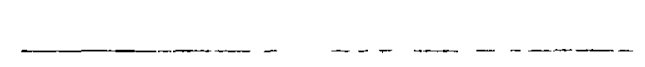

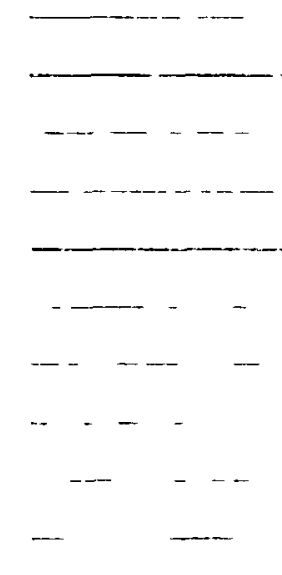

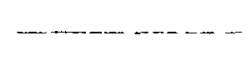

$-+-\cdots$ 
LAB. NO. An OCD WEPTOT TOO

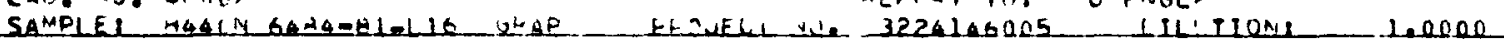

DATE: $2-\overline{23-77}$ PLAIt NOE 7I-0I

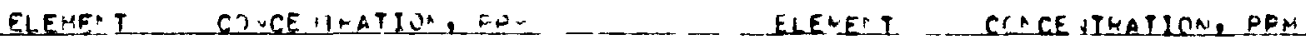

ELEMENT - CONCENTRAIIONS PPM

\begin{tabular}{|c|c|c|c|c|c|c|}
\hline & $\begin{array}{l}\Delta G \\
H B\end{array}$ & $\begin{array}{r}.511 \\
21.02 \\
\end{array}$ & QL. & $\therefore \quad 1.00$ & $\begin{array}{l}0 \\
\text { aI }\end{array}$ & $\begin{array}{r}2.00 \\
1.00 \\
\end{array}$ \\
\hline & CA & $\begin{array}{r}40.6 n \\
<\quad 4060 \\
\end{array}$ & $\begin{array}{l}C^{13} \\
C Q\end{array}$ & $\begin{array}{r}10.00 \\
\times \quad 1.00 \\
\end{array}$ & $\begin{array}{l}\text { CE } \\
C S\end{array}$ & $\begin{array}{l}80.00 \\
<\quad 10.00 \\
\end{array}$ \\
\hline & $\begin{array}{l}\text { rU } \\
\text { EU }\end{array}$ & $\begin{array}{r}1.00 \\
<\quad 200.00 \\
\end{array}$ & or & $\begin{array}{r}20.00 \\
10.00 \\
\end{array}$ & $\begin{array}{l}E R \\
G C\end{array}$ & $\begin{array}{r}6000 \\
\times \quad 10.00\end{array}$ \\
\hline & $\begin{array}{l}H F \\
K\end{array}$ & $\begin{array}{r}20.00 \\
6.00 \\
\end{array}$ & Ho & $\therefore \quad 10000$ & In & $\begin{array}{r}1.00 \\
\end{array}$ \\
\hline & $\begin{array}{l}\mathrm{LO} \\
10 \\
\end{array}$ & $<\quad .50$ & $\begin{array}{l}\text { MG } \\
N A\end{array}$ & $\begin{array}{r}10.00 \\
\times \quad 0.00 \\
\end{array}$ & $\begin{array}{l}M N \\
N B\end{array}$ & $\begin{array}{r}1.00 \\
<\quad 6.00\end{array}$ \\
\hline $\begin{array}{ll}0 & 1 \\
1 & 1\end{array}$ & $\begin{array}{l}\text { ND } \\
P E\end{array}$ & $\begin{array}{r}60.00 \\
<\quad 5.00 \\
\end{array}$ & $\begin{array}{l}N I \\
P R\end{array}$ & $\therefore \quad \begin{array}{r}400 \\
\end{array}$ & PR & $\begin{array}{r}600.00 \\
\times \quad 6.00 \\
\end{array}$ \\
\hline a & $\begin{array}{l}\text { SP } \\
\text { Sil }\end{array}$ & $\begin{array}{r}8.10 \\
<\quad 100.00 \\
\end{array}$ & $\begin{array}{l}\text { SC } \\
\text { SN. }\end{array}$ & $\begin{array}{r}1.00 \\
<\quad 6,00 \\
\end{array}$ & $\begin{array}{l}5 I \\
58\end{array}$ & $\begin{array}{r}80000 \\
\times \quad \text { cedere } \\
\end{array}$ \\
\hline$\cdots$ & IA & $\begin{array}{r}40.00 \\
<\quad 20.00 \\
\end{array}$ & $\begin{array}{l}T B \\
\text { TH } \\
\end{array}$ & $\begin{array}{r}+0.00 \\
.00 \\
\end{array}$ & $\begin{array}{l}T I \\
y\end{array}$ & $\begin{array}{r}20.00 \\
20.00 \\
\end{array}$ \\
\hline & $2 t$ & $\begin{array}{r}40.00 \\
2.06\end{array}$ & $Y B$ & 10.00 & $2 N$ & $<10.00$ \\
\hline
\end{tabular}

CONGEITRATION DASEO OA TRGINAL SAMPLE AEFTKE DILUTTON WITH UILUENT

> MEANS BHEAIER THAN

CEANS LESS THAN THE SENSITIVITYOF THE SPECTHOGRAPHIC PROCEOURE USEO

RESILTS APE CORRECT WITHIN FACTON OF 4 ON, (CNE SIAYHARD OEVIATION) 


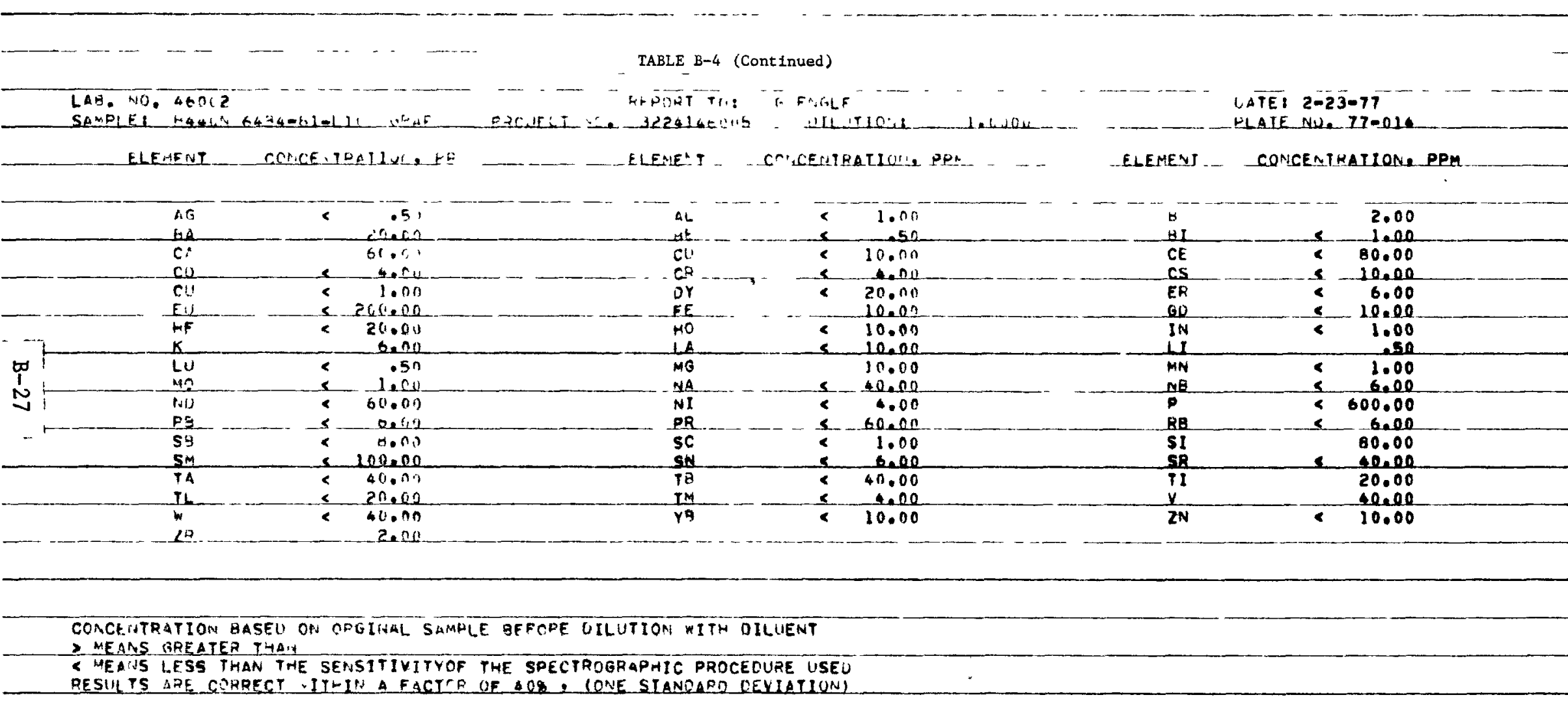




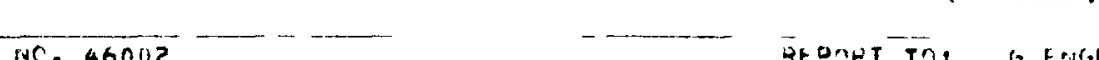

LAB. NC. 46RIZ

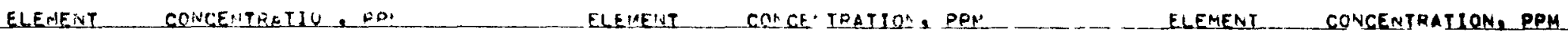

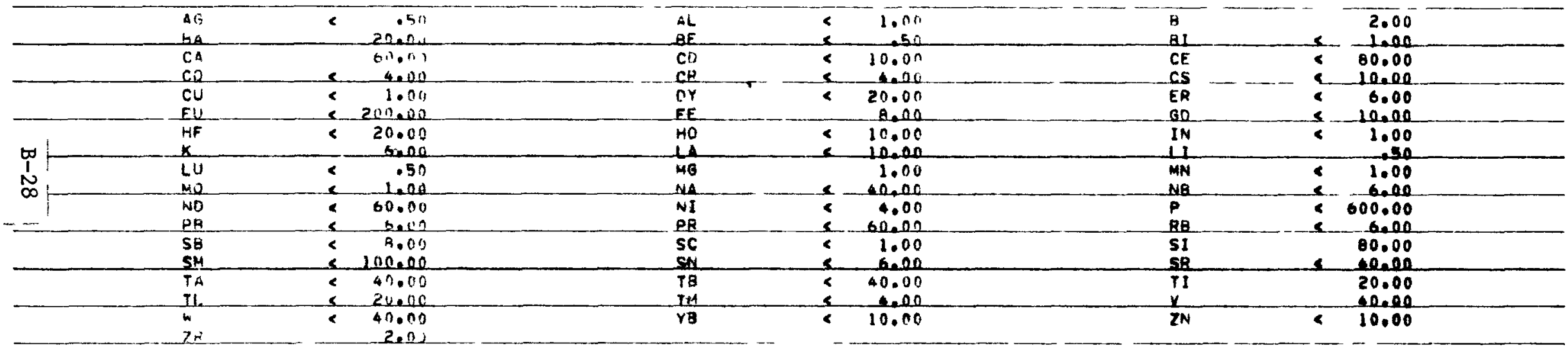

CONCFNTRATION BASED CV ORGINAL SAMFLE FEFORE OILUTION ITH DILUENT

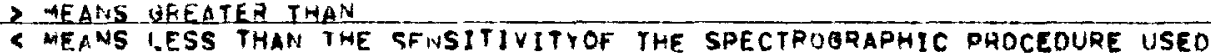

RESULTS ARE CORRECT ITHI A A FACICP OE LOX, CONE SIANDARO CEVIGLLON 


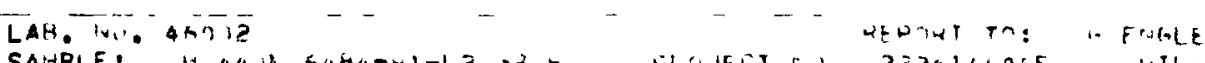

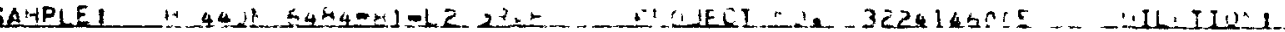

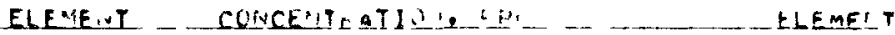
ELEMERT - CQNCENTHATION, PPM
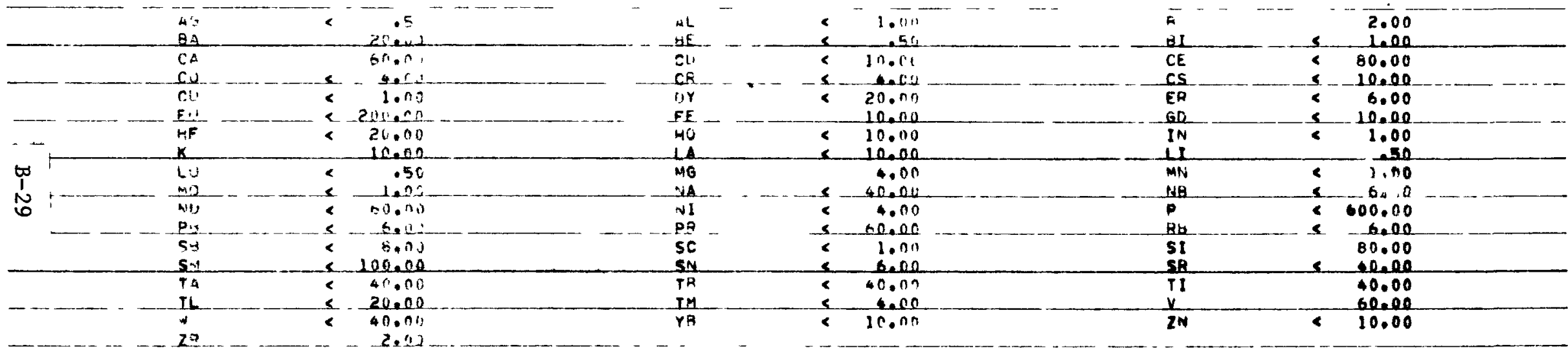

CONCENTRATION DASED ON OFGINAL SANPLE REFORE DILUTION WITM DILUENT

$\rightarrow$ MEANS GPEATER TMATH

C MEANS LESS THAN THE SENSITIVITYOF THE SPECTROGRAPHIC PROCEDUAE USED

AESIILTS ARE CDRPECT ITHIP G EACTCE OF AOX L IONE SIANIDARE OEVIAIION) 
APPENDIX C

GRADE PGX DATA

This appendix contains complete data sets for the bulk density, tensile properties, flexural strength, compressive properties, and chemical impurity content of grade PGX graphite. 
TABLE $C-1$

TENSILE PROPERTIES OF PGX GRAPHITE

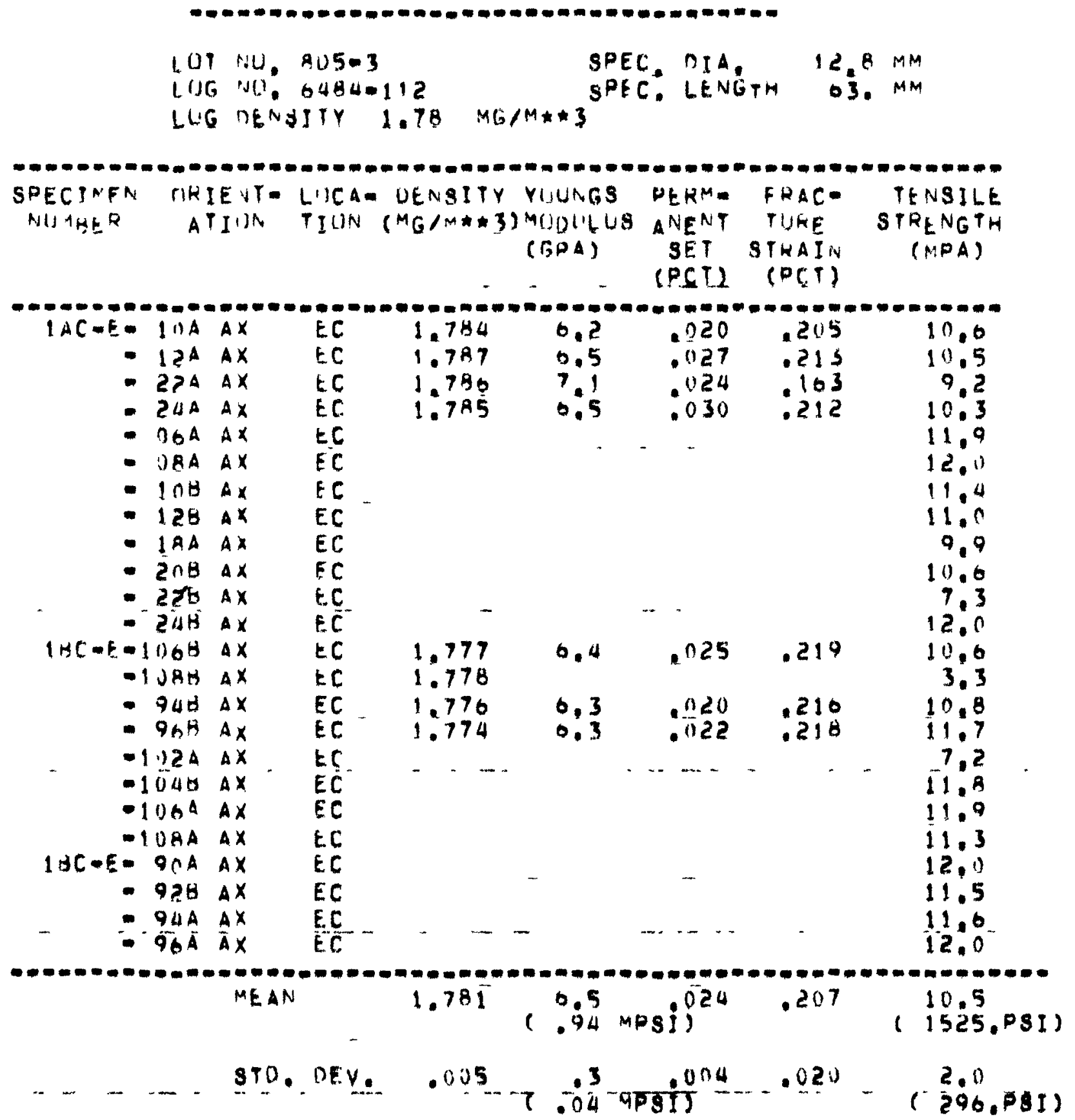




\section{TABLE C-1 (Continued)}

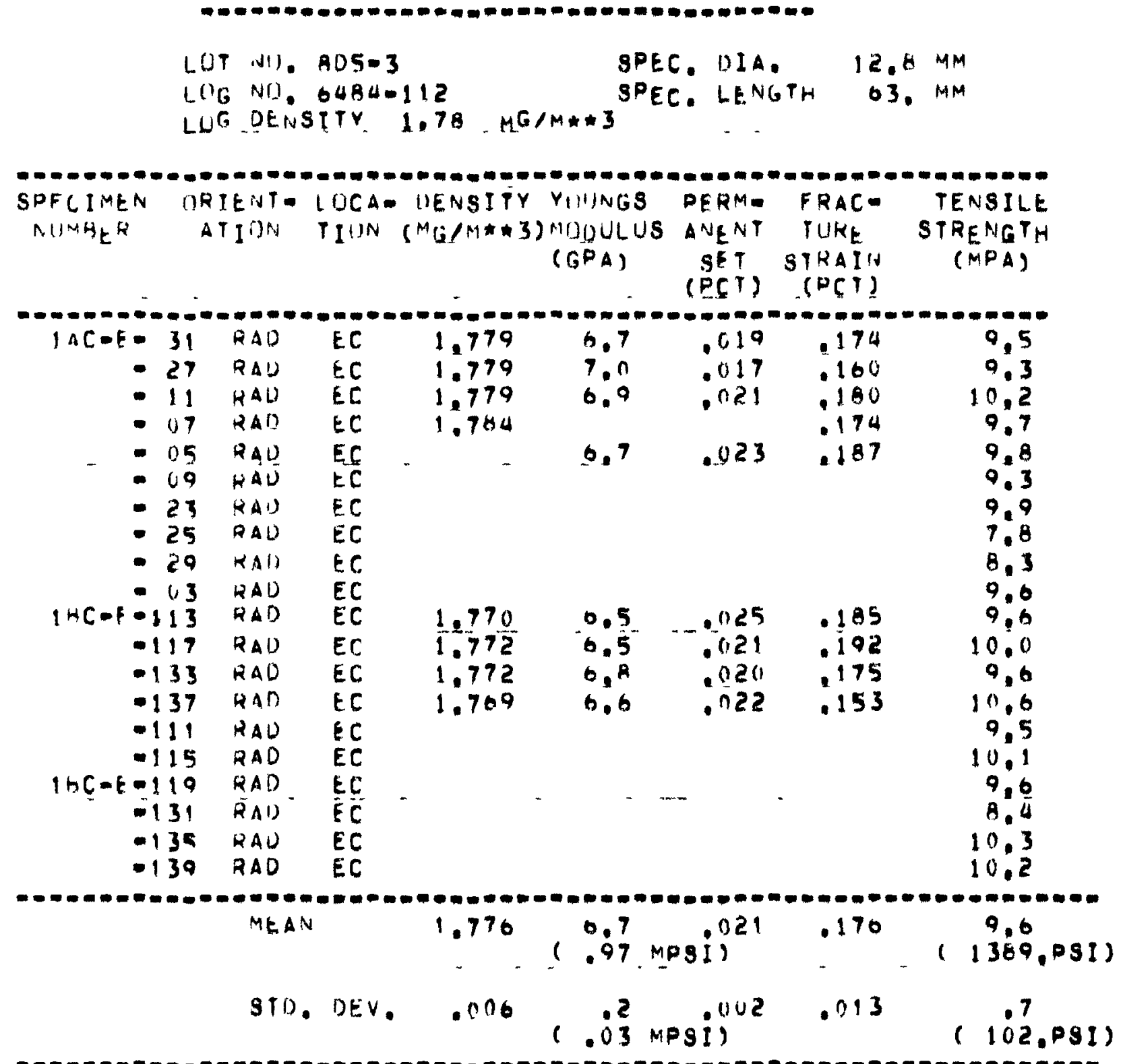


TABLE C-1 (Continued)

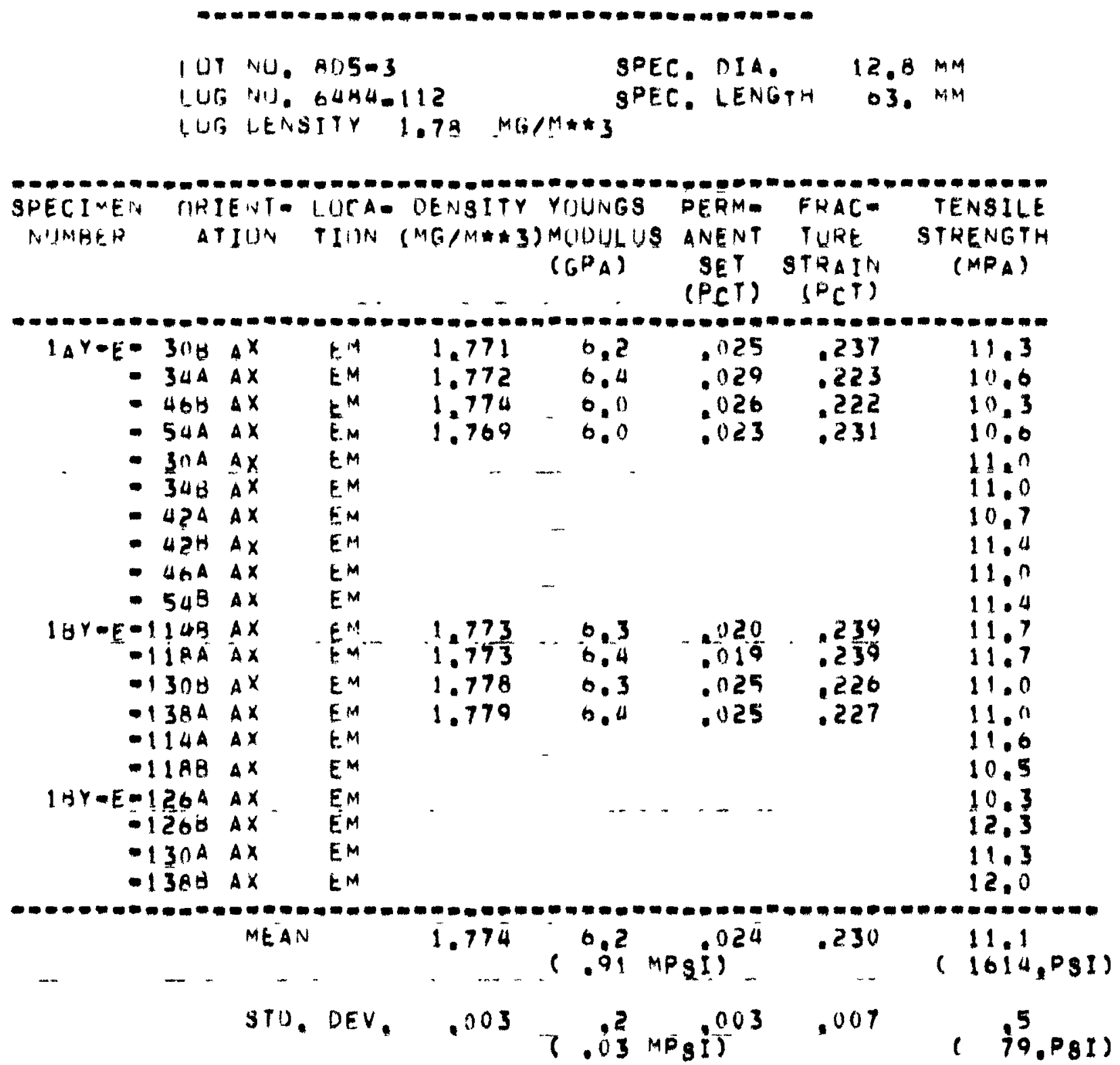

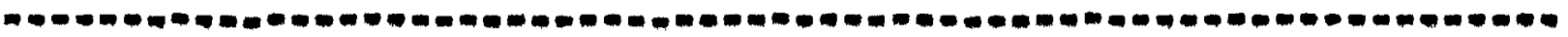


TABLE C-1 (Continued)

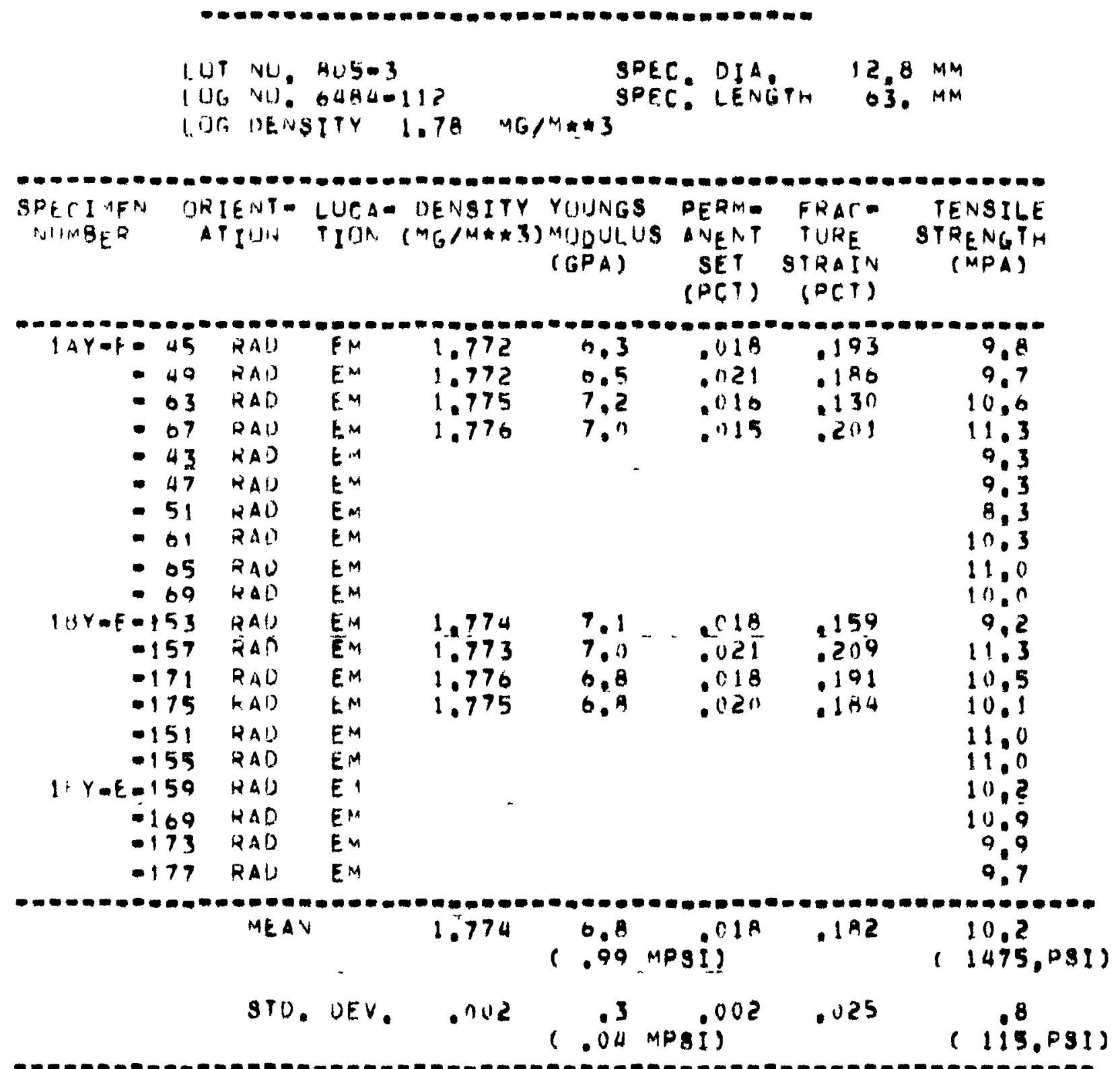


TABLE C-1 (Continued)

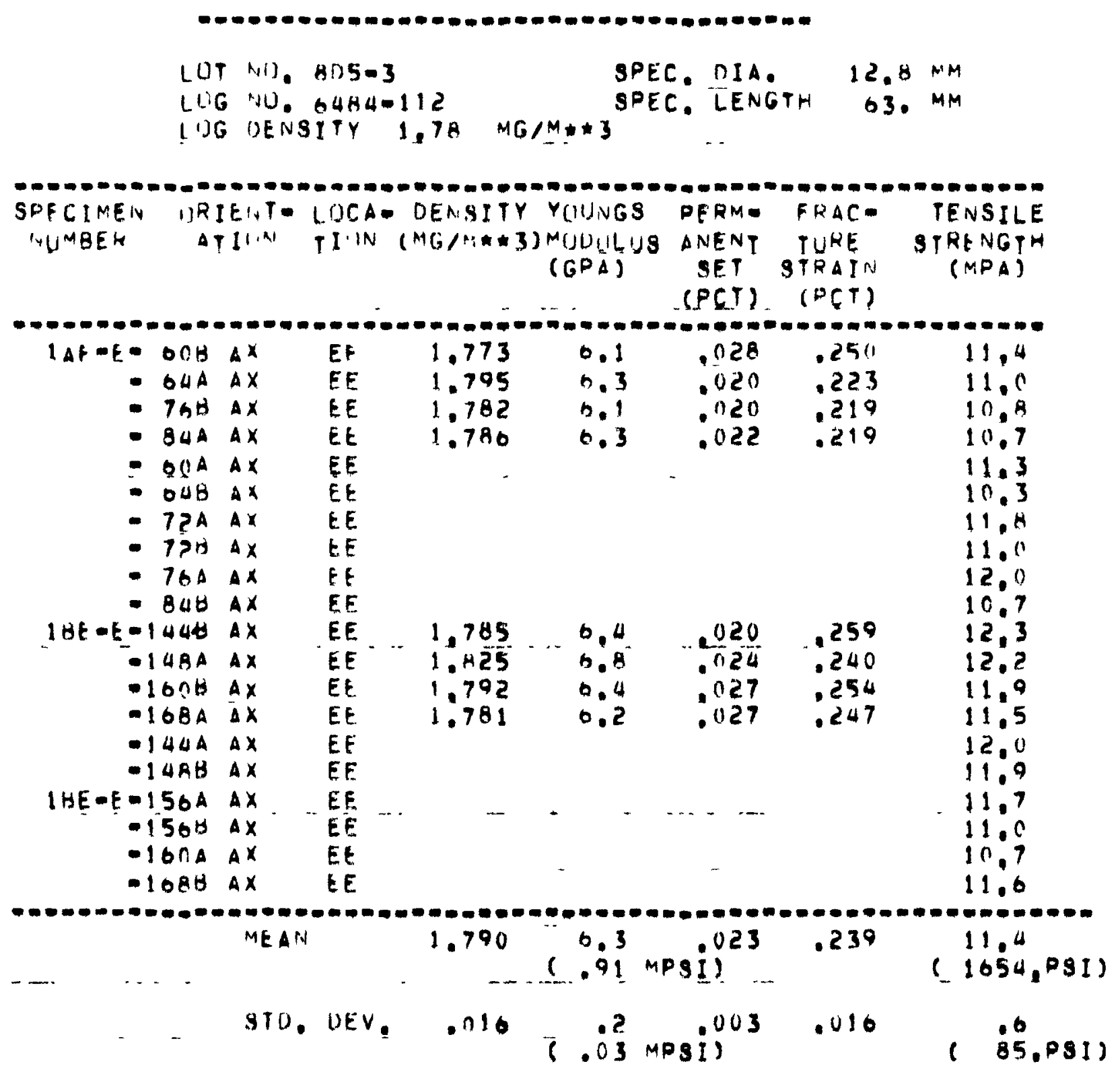

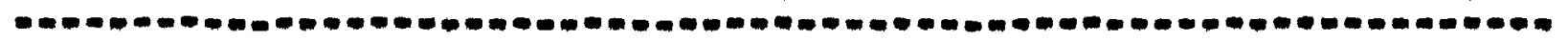


TABLE C-1 (Continued)

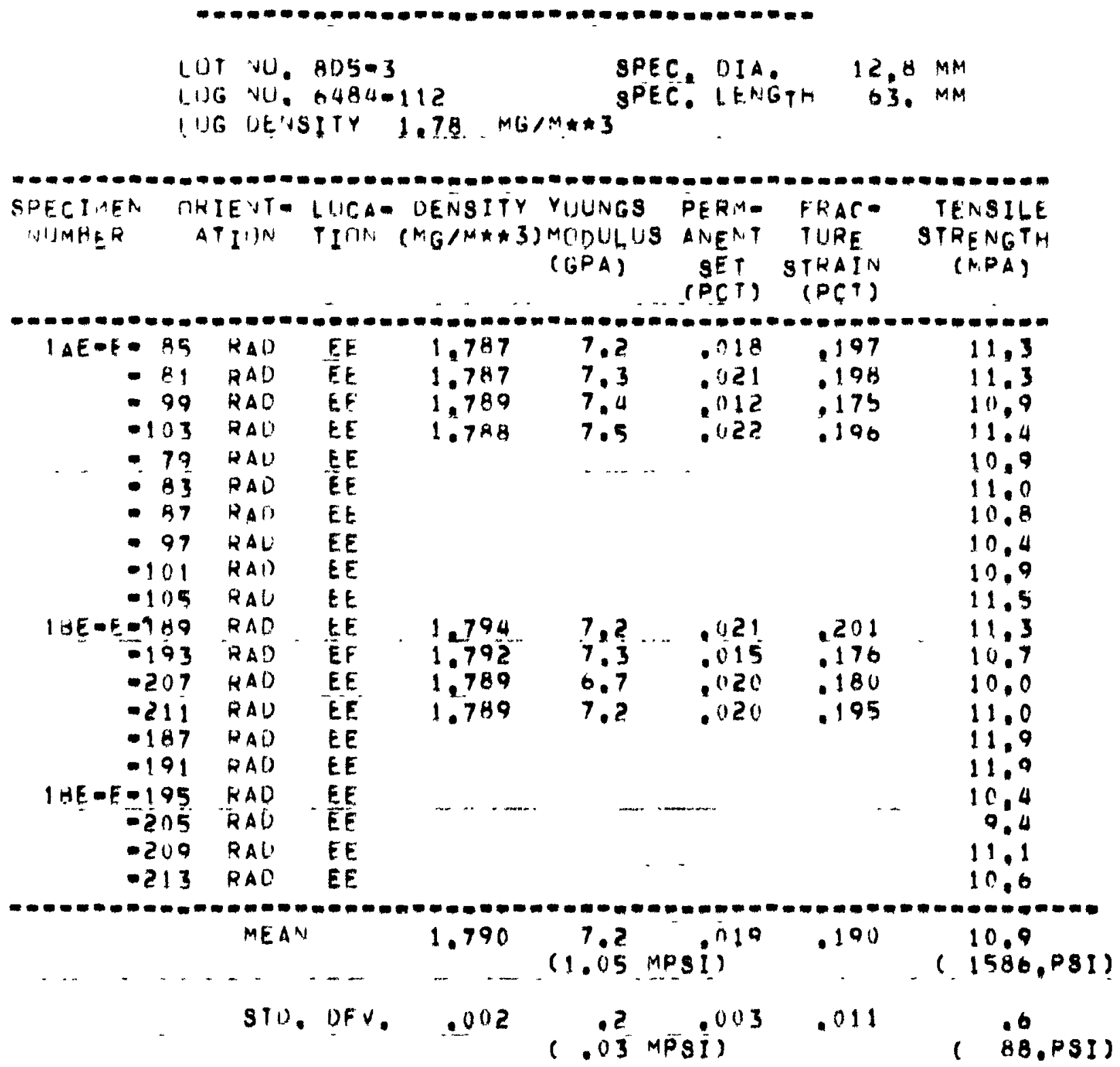

- 
TABLE C-1 (Continued)

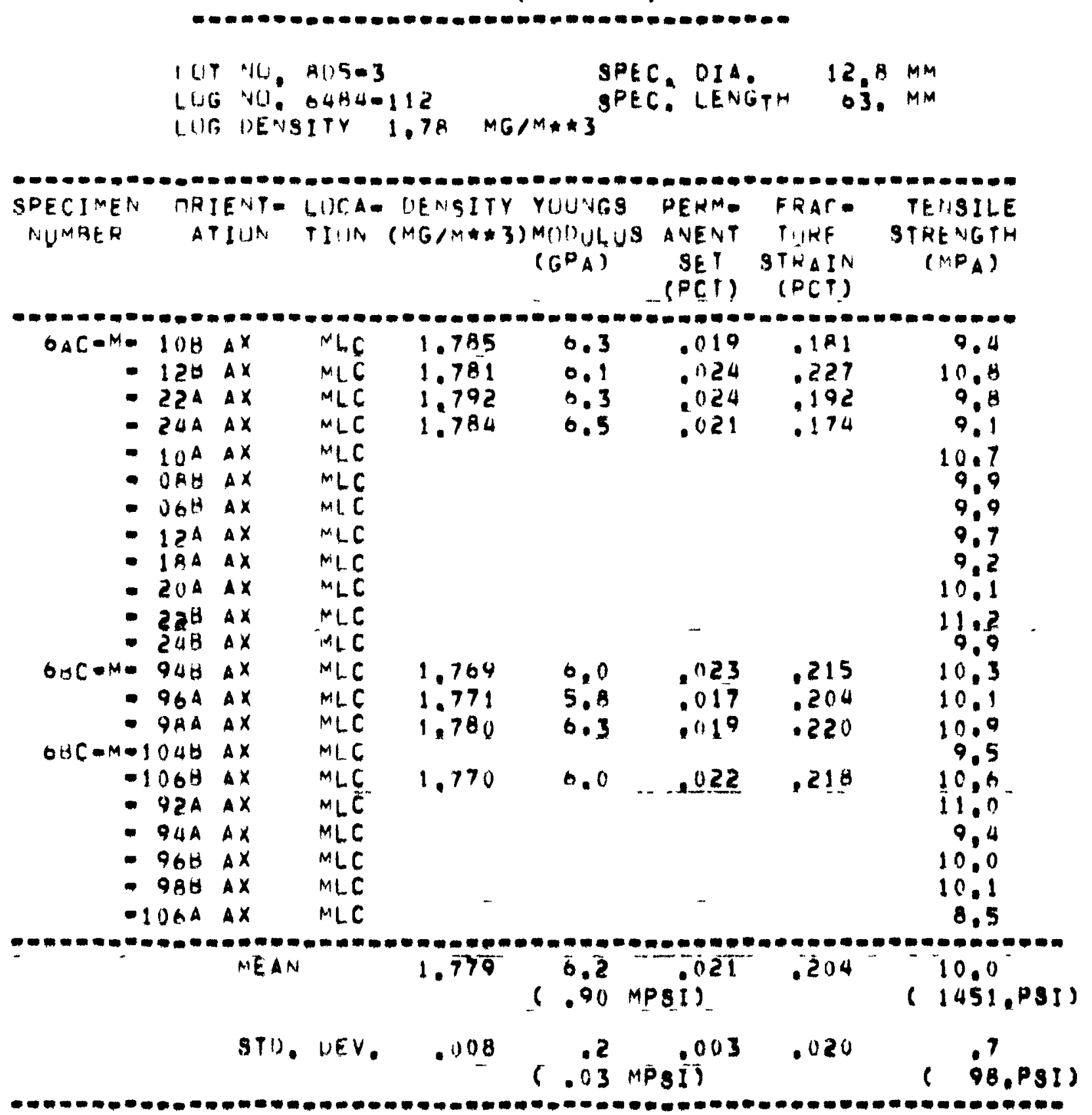




\section{TABLE C-1 (Continued)}

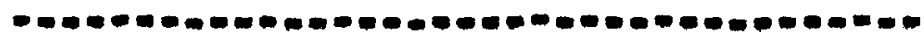
LIT NO, $\$ 05-3$
SPEC: OIA.
$12.8 \mathrm{MM}$
LUG NU. $6484-112$
IOG OENSITY
$1.78 \mathrm{M}(\mathrm{i} / \mathrm{1} * 3$
SPEC. LENGTH
b3. $M M$

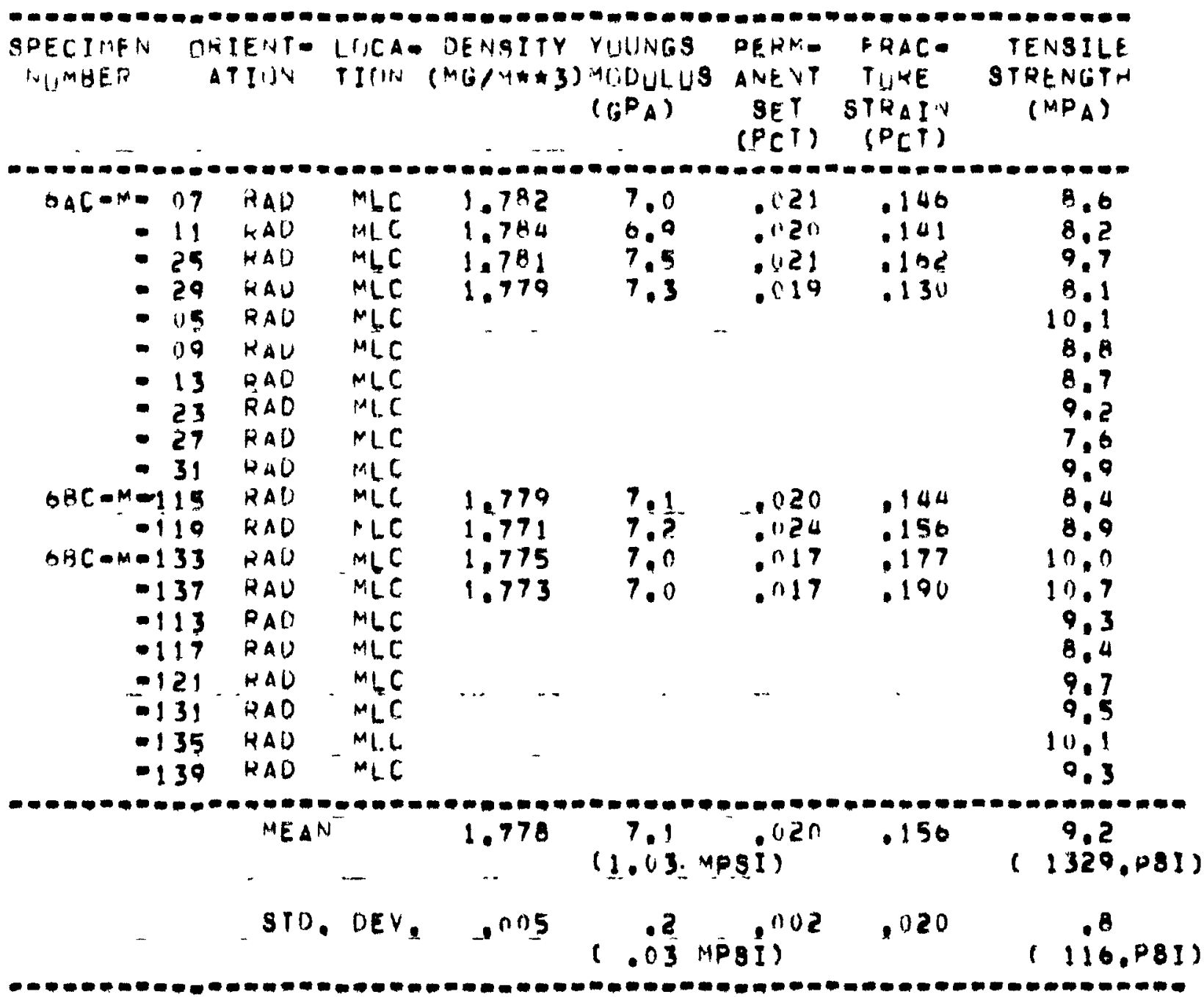


TABLE C-1 (Continued)

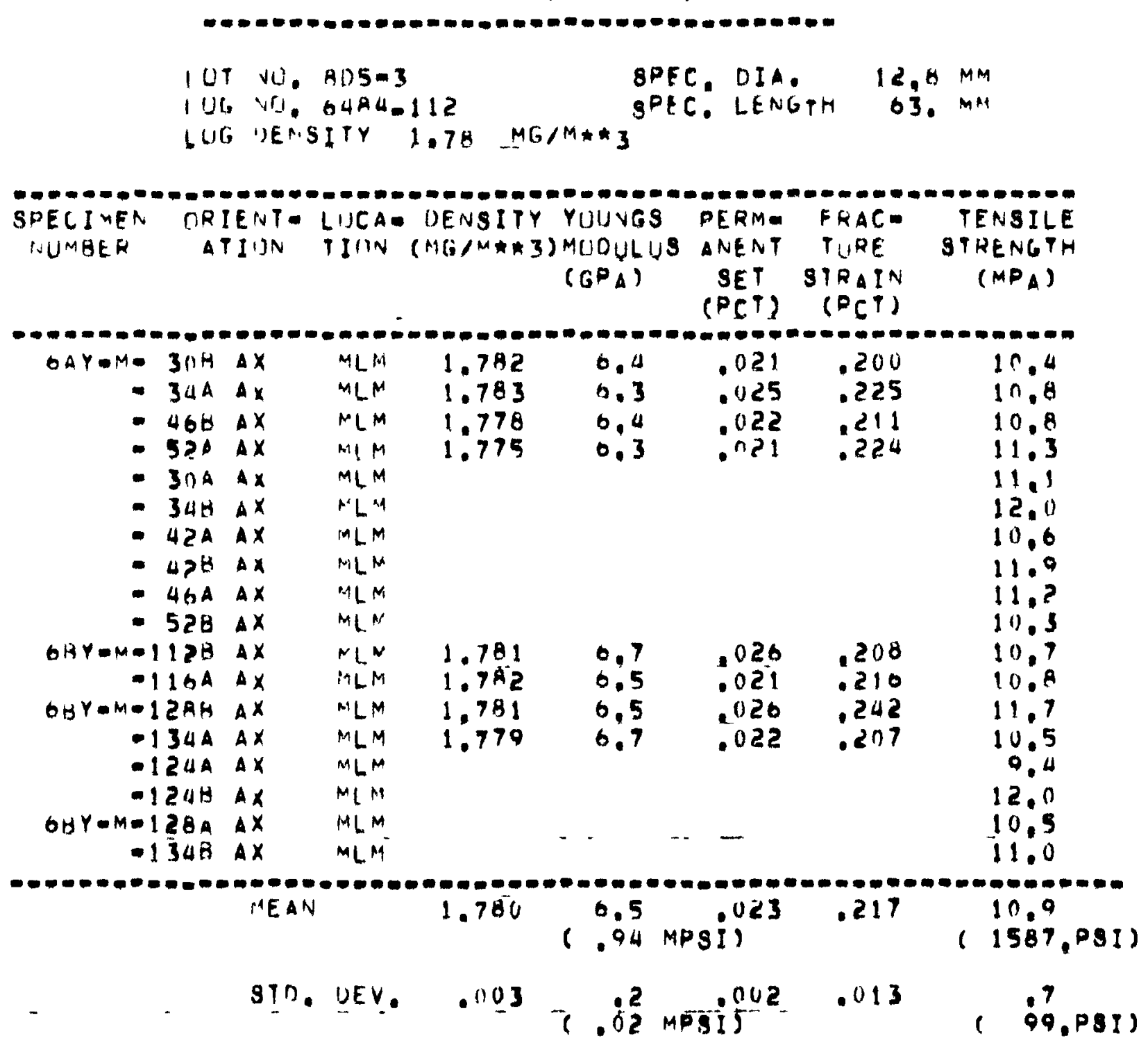

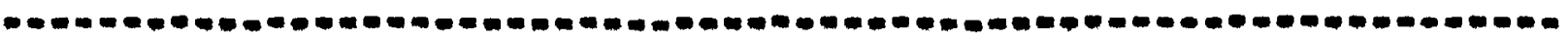


TABLE C-1 (Continued)

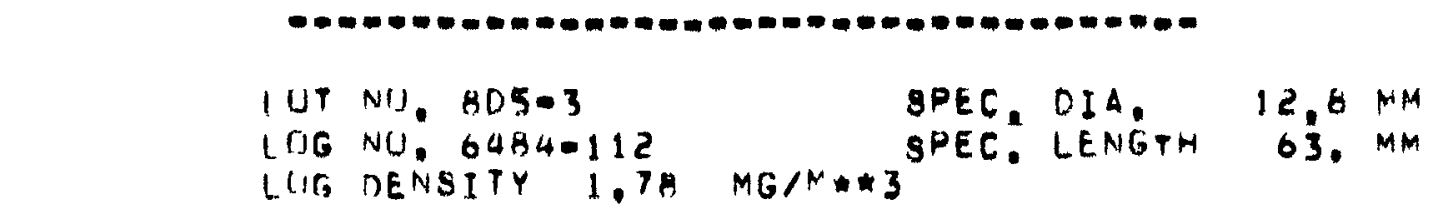

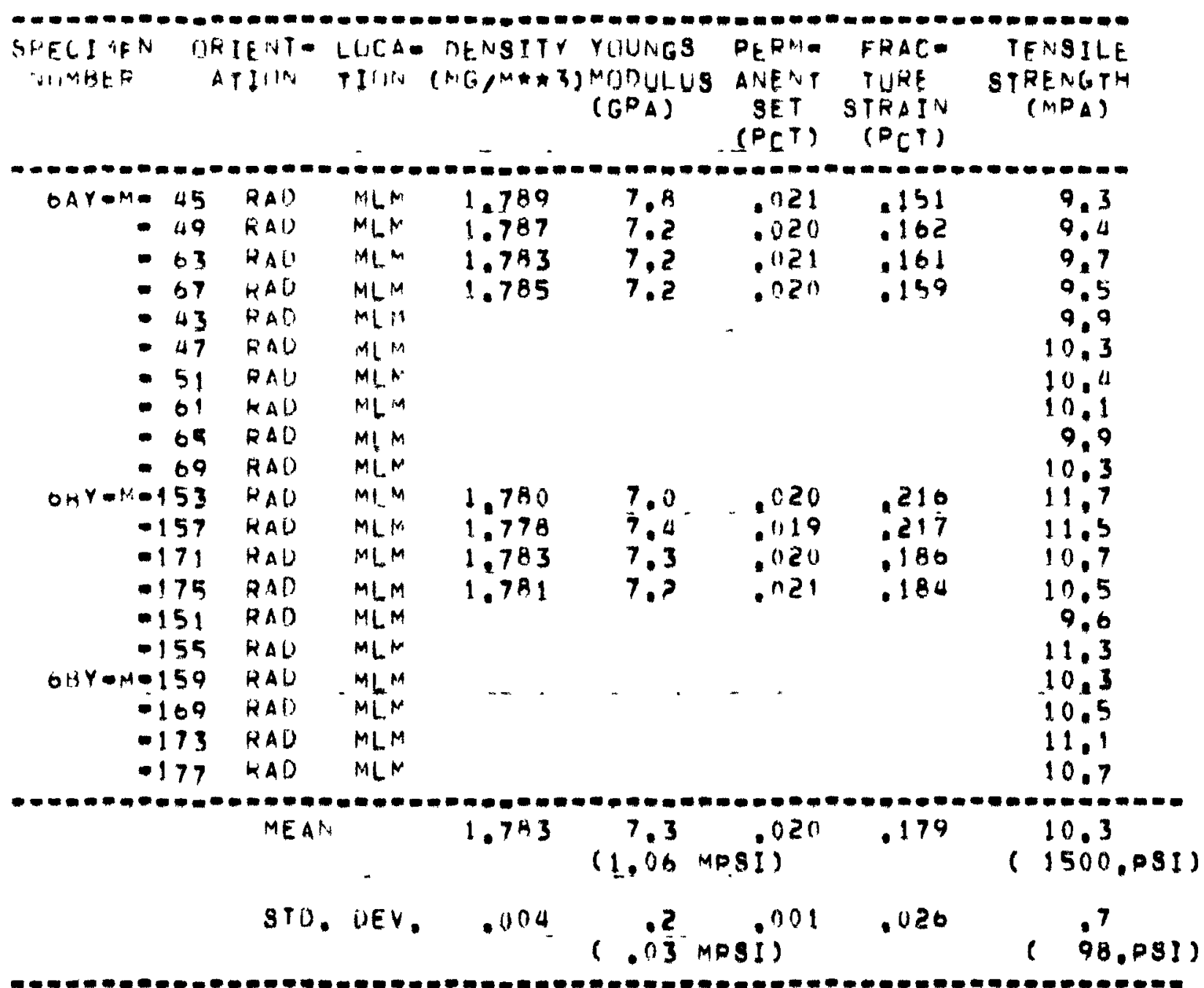


TABLE C-1 (Continued)

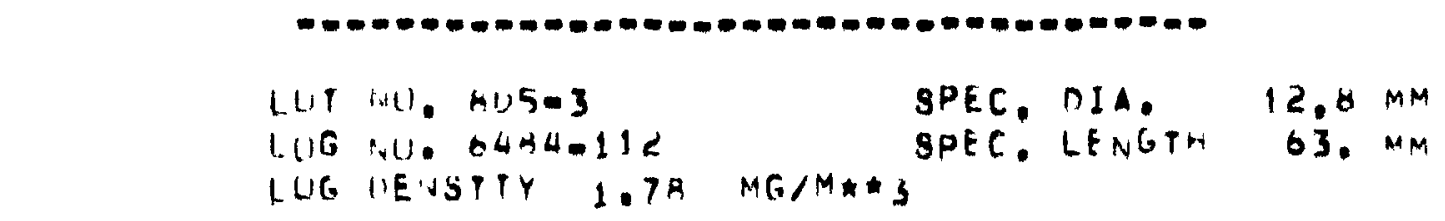

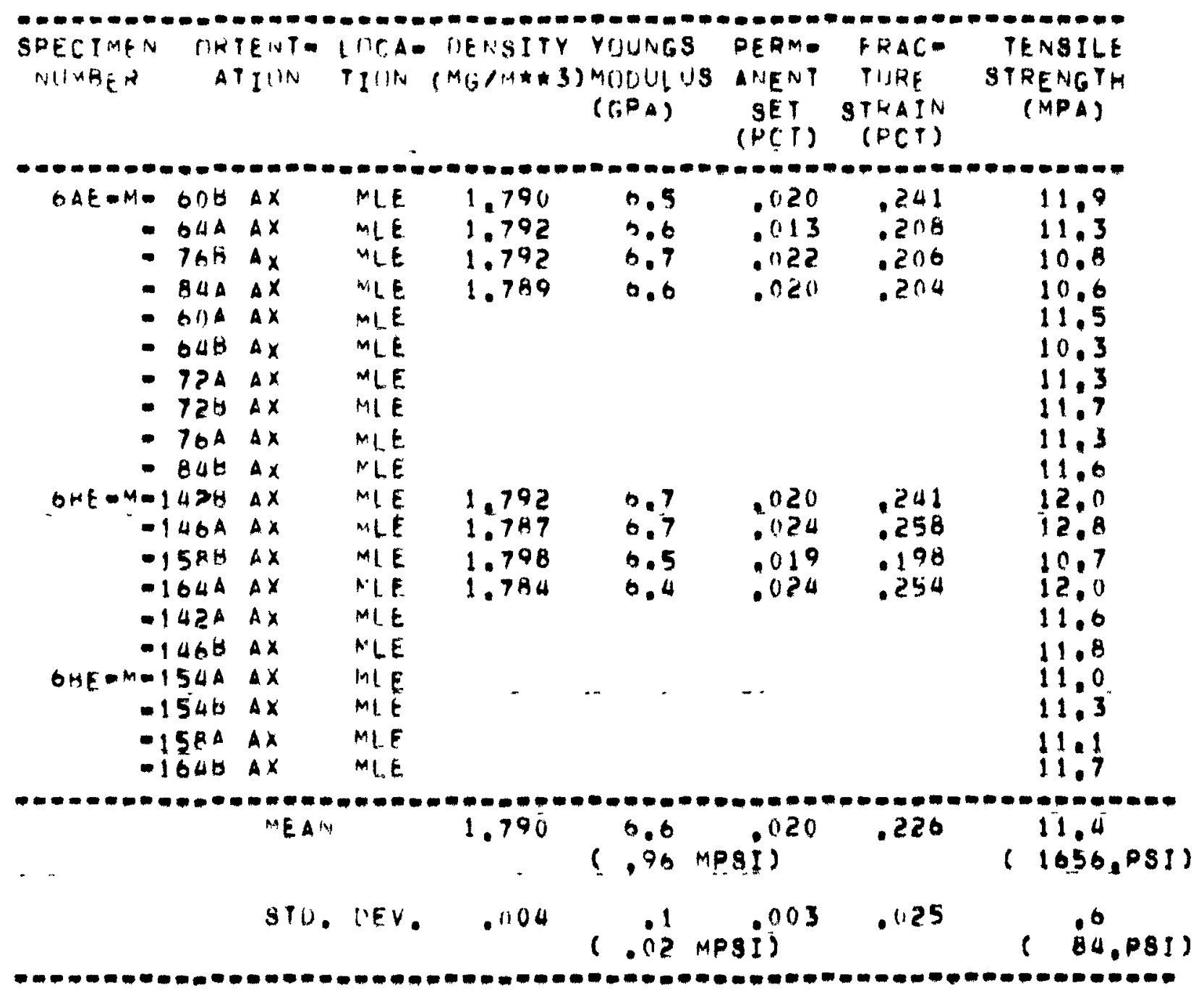


TABLE C-1 (Continued)

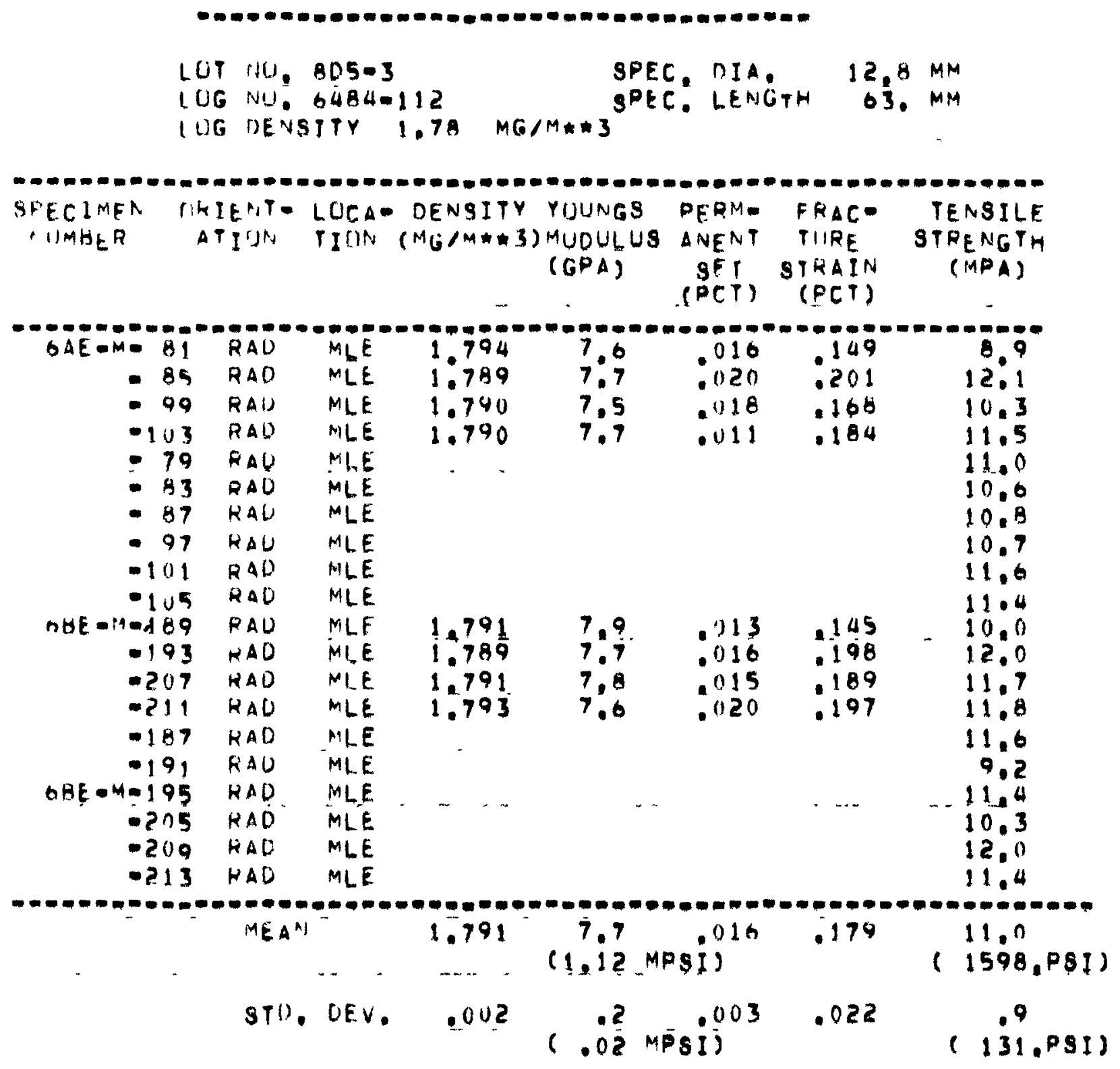


TABLE C-1 (Continued)

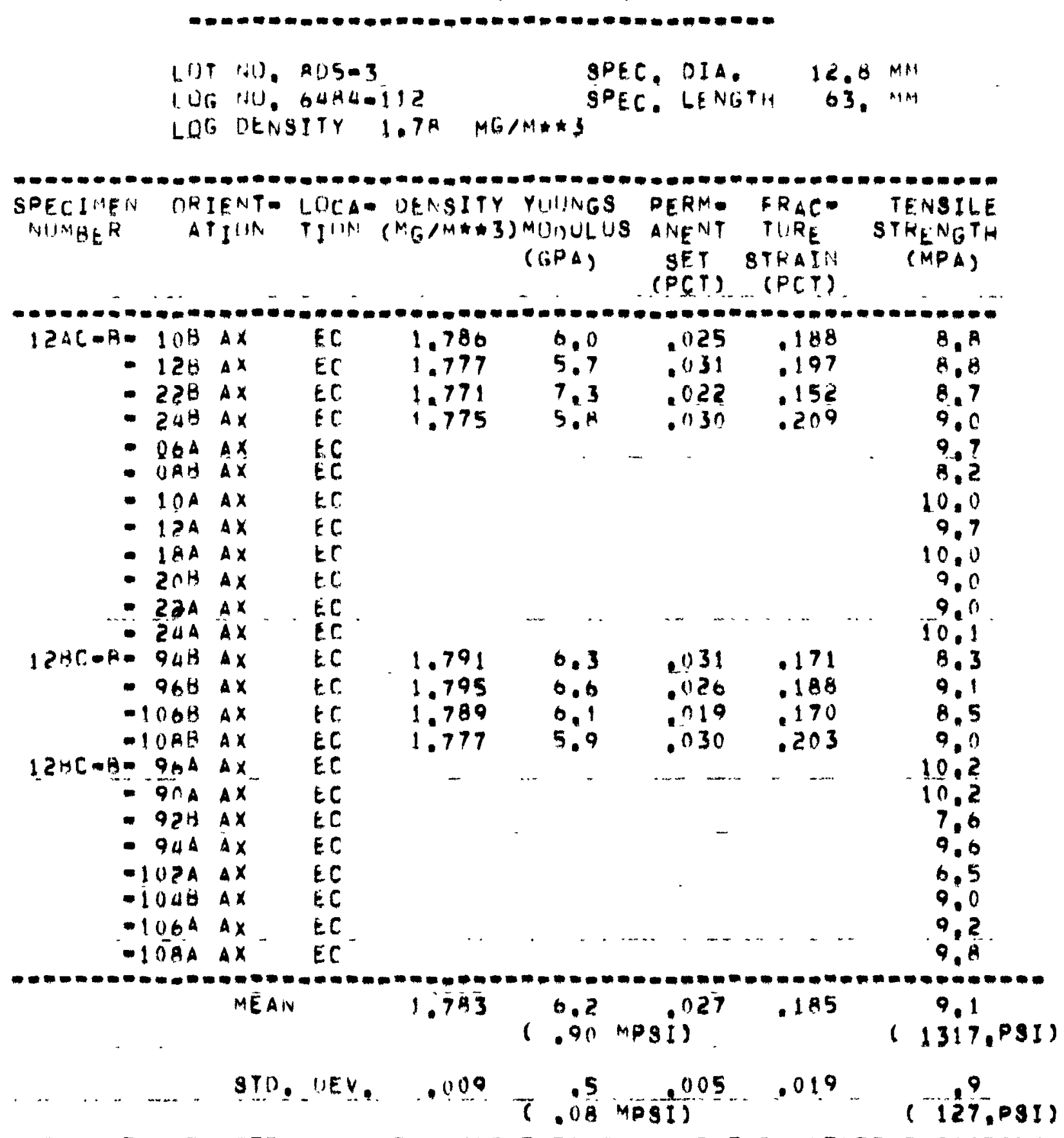


TABLE C-1 (Continued)

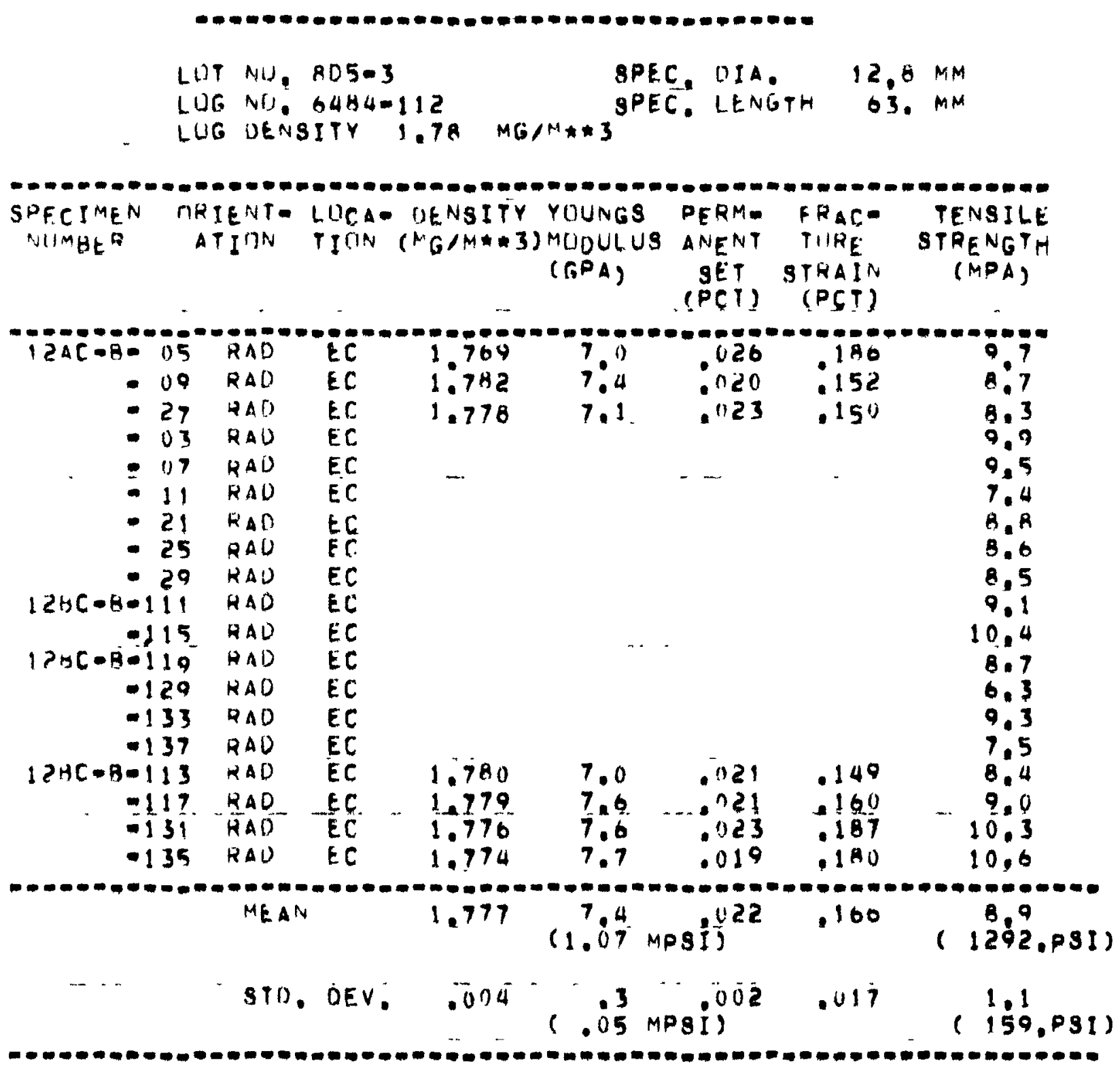


TABLE C-1 (Continued)

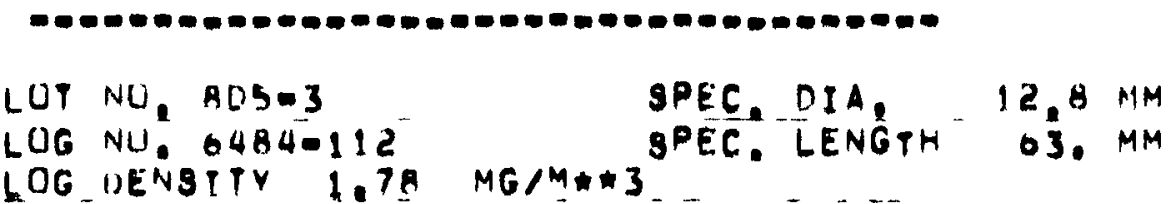

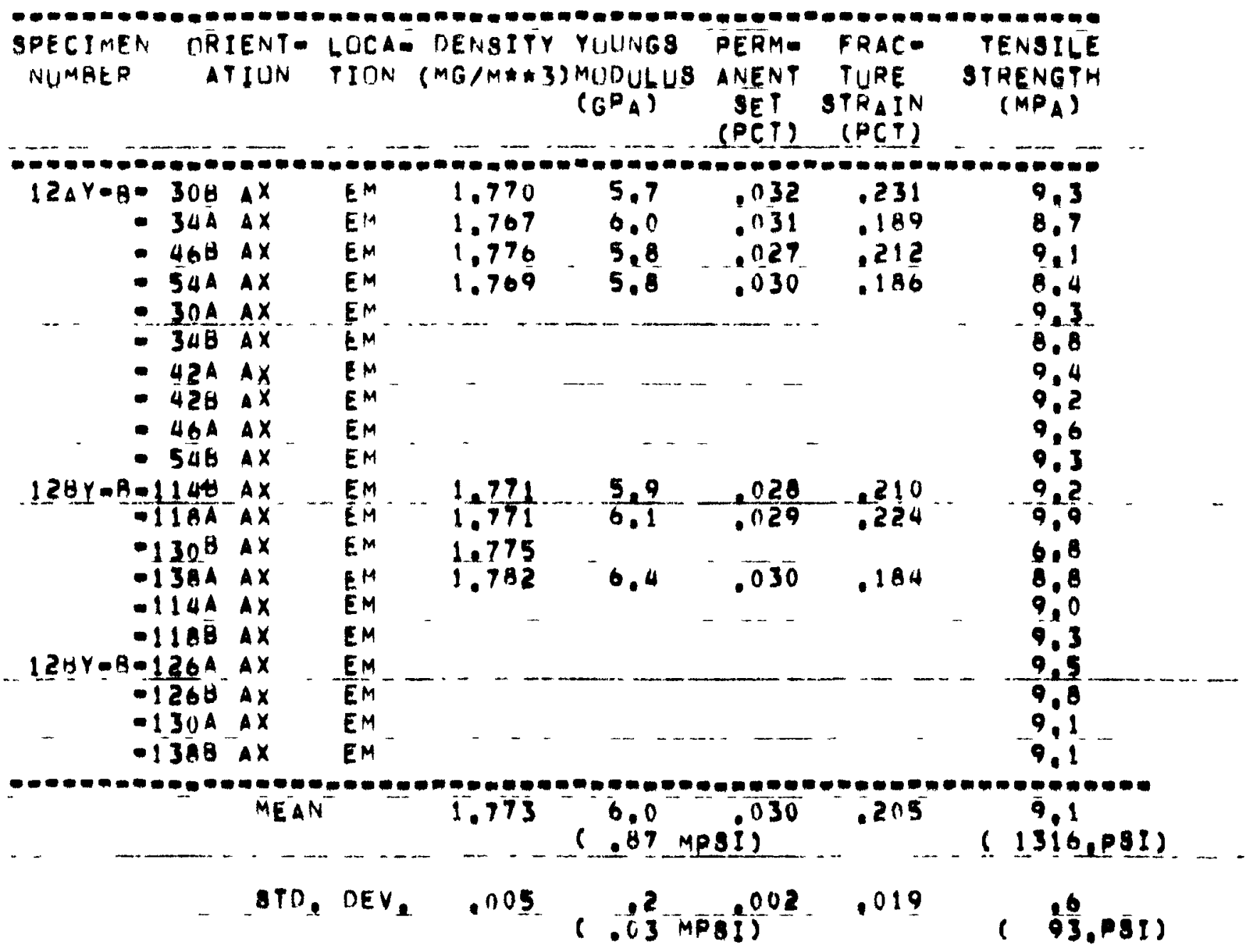


TABLE C-1 (Continued)

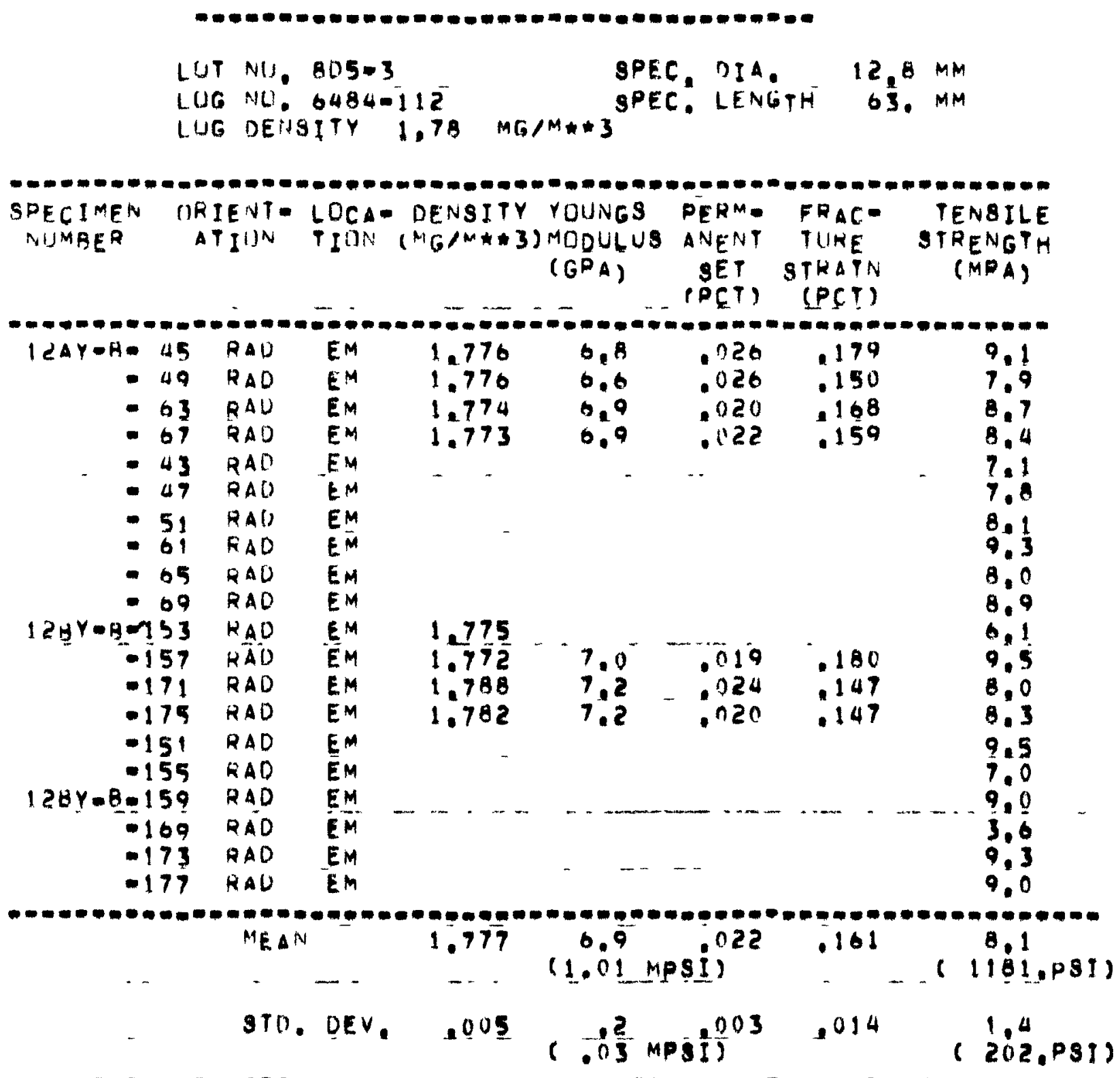




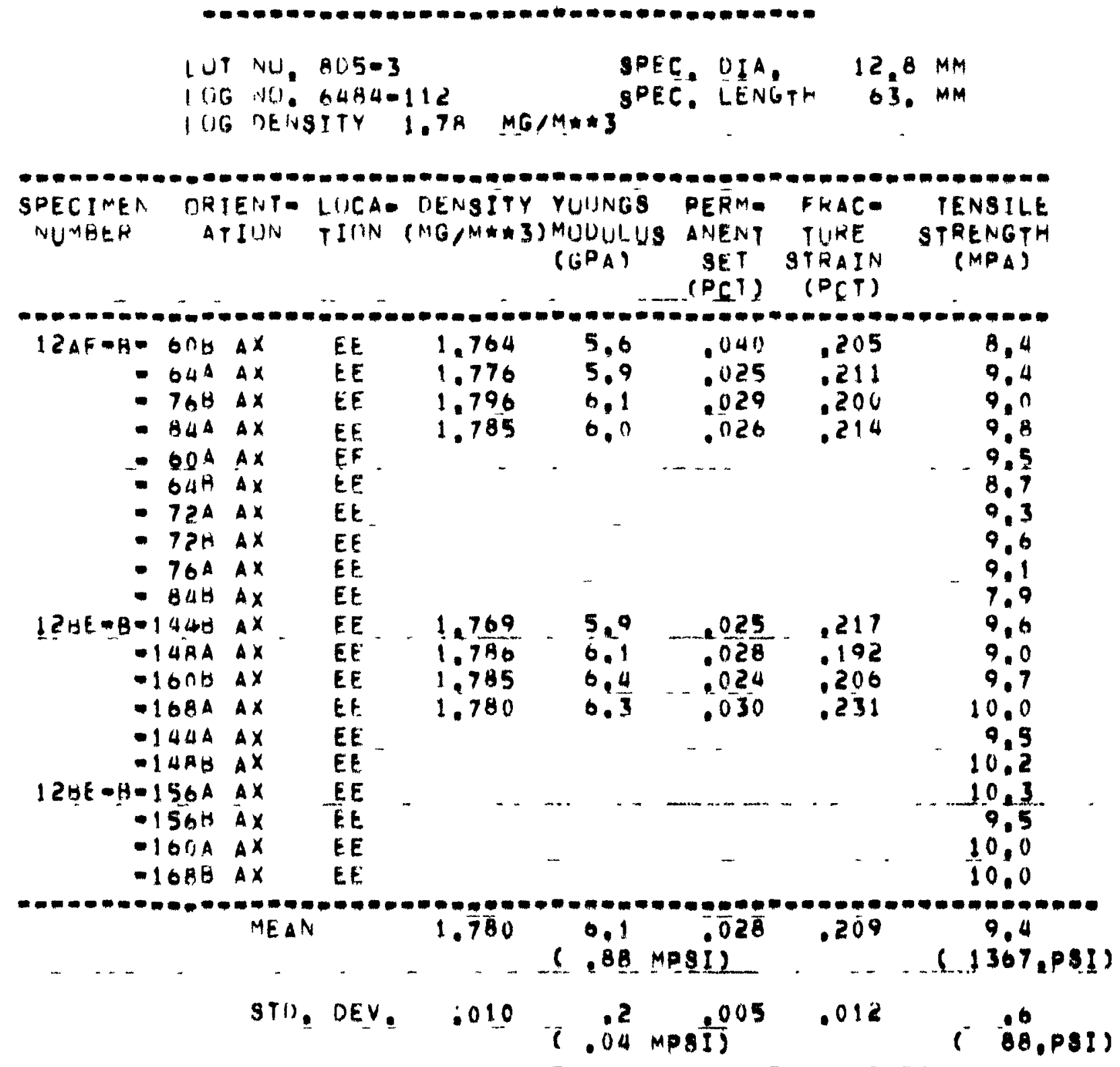


TABLE C-1 (Continued)

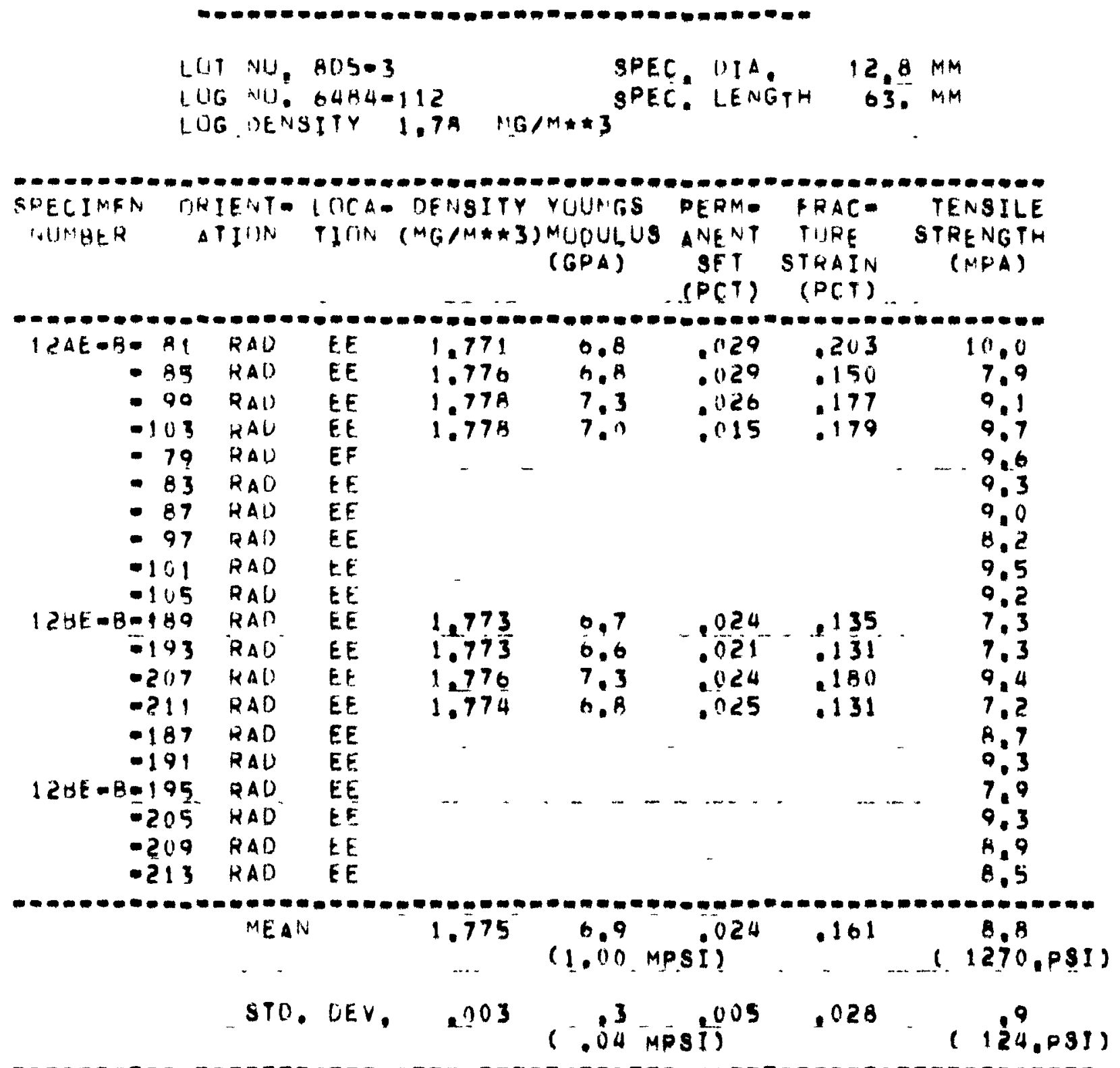


TABLE $\quad \mathrm{C}-2$

FLEXURAL STRENGTH OF PGX GRAPHITE

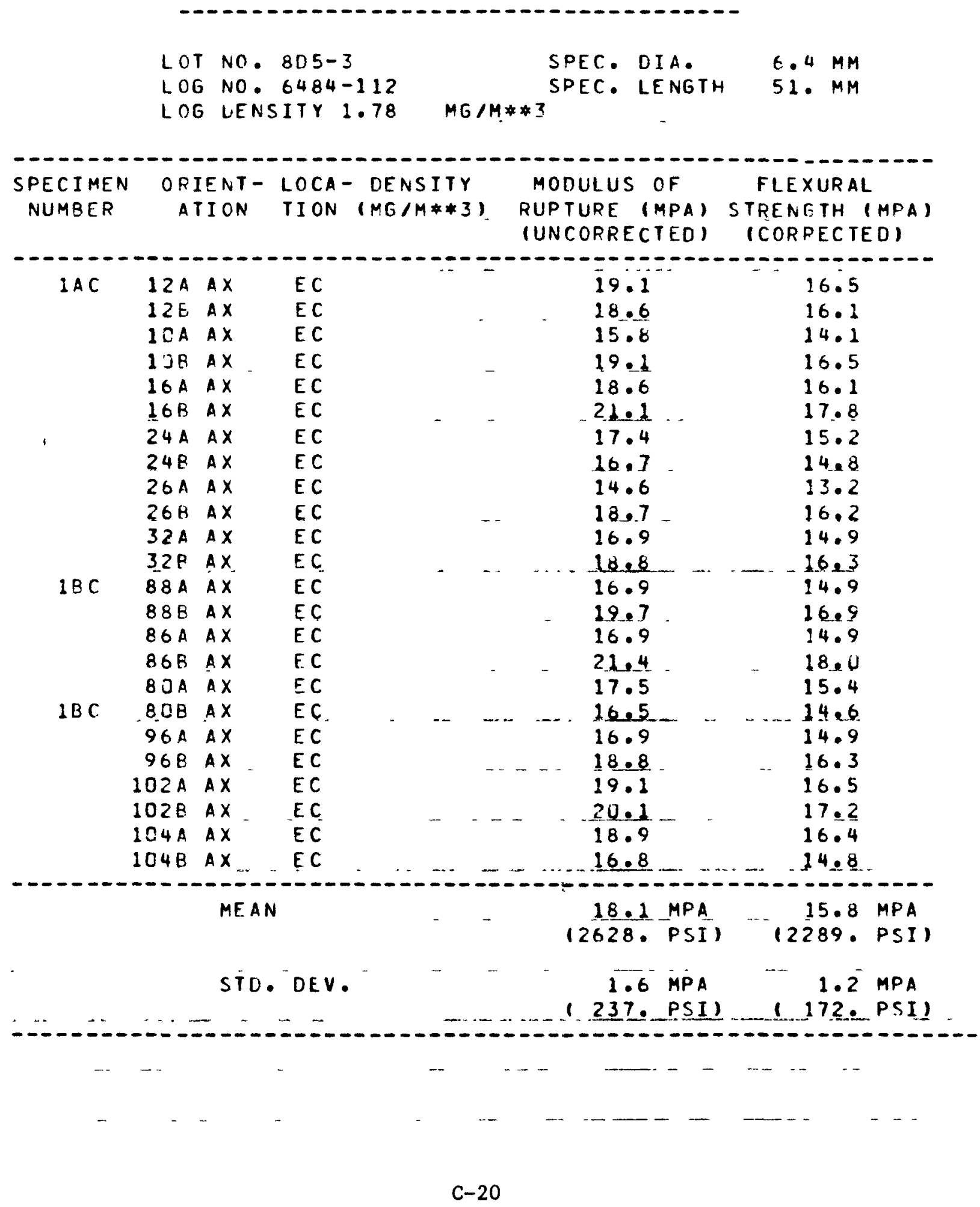


TABLE $\mathrm{C}-2$ (Continued)

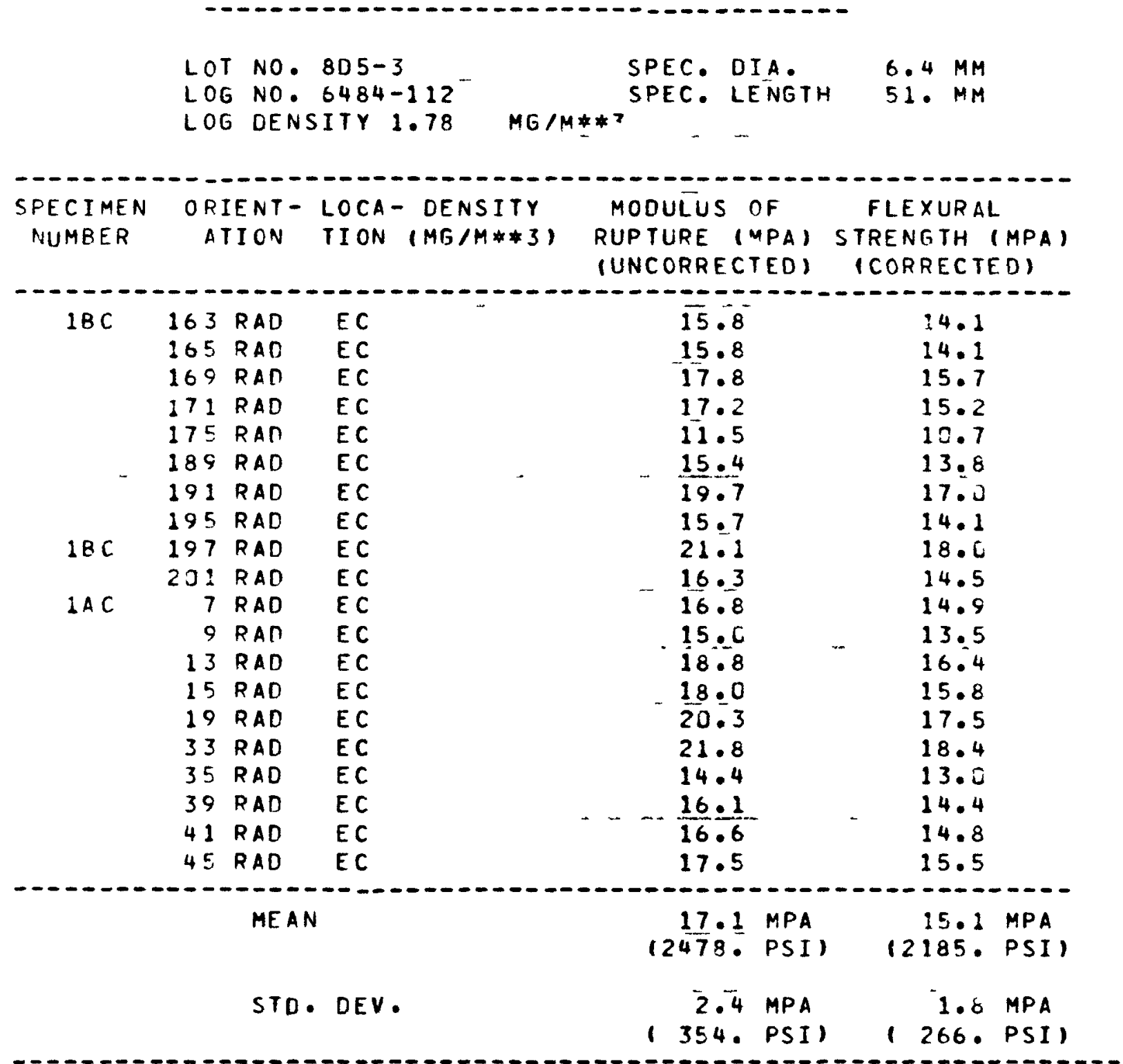


TABLE C-2 (Continued)

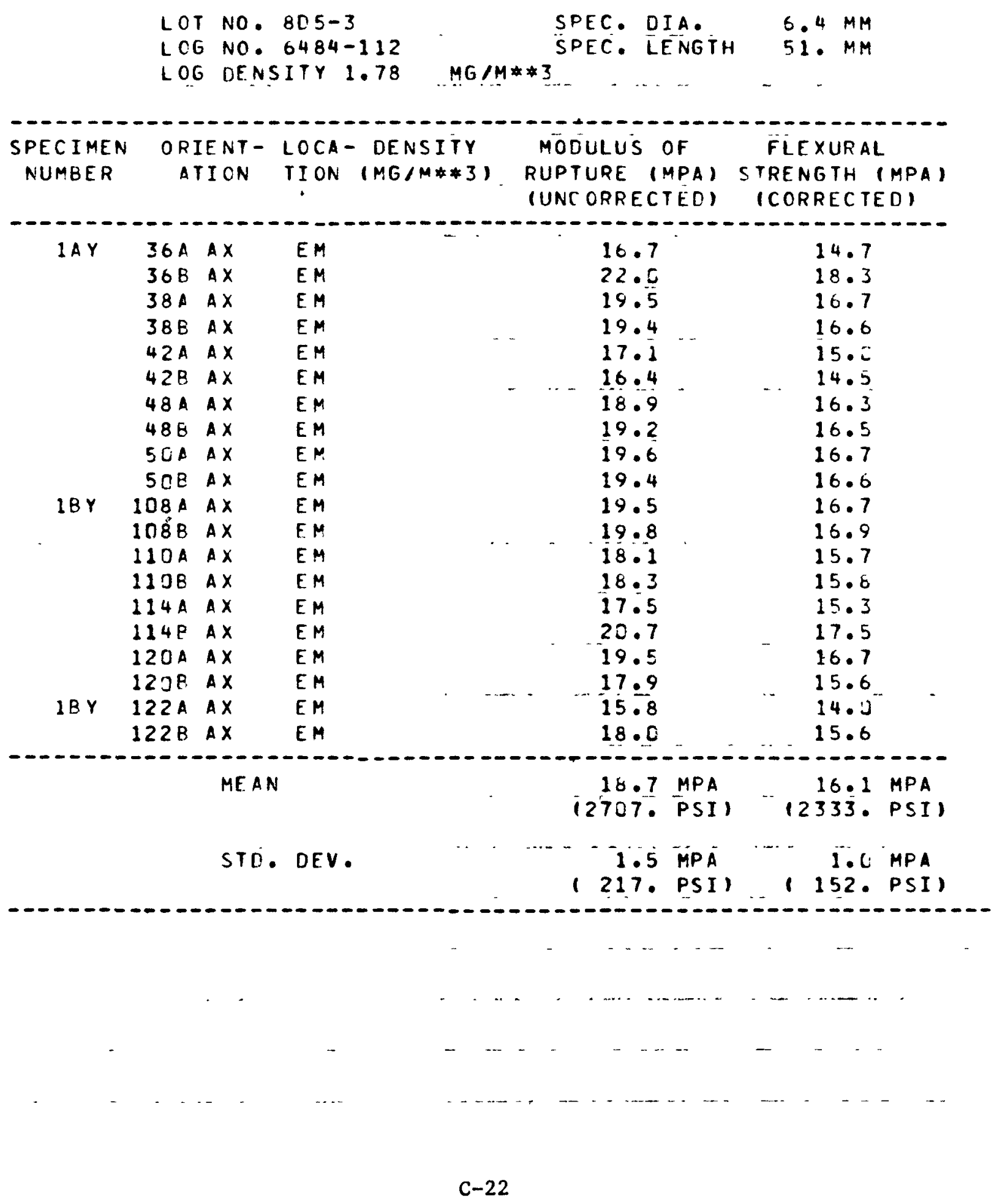


TABLE C-2 (Continued)

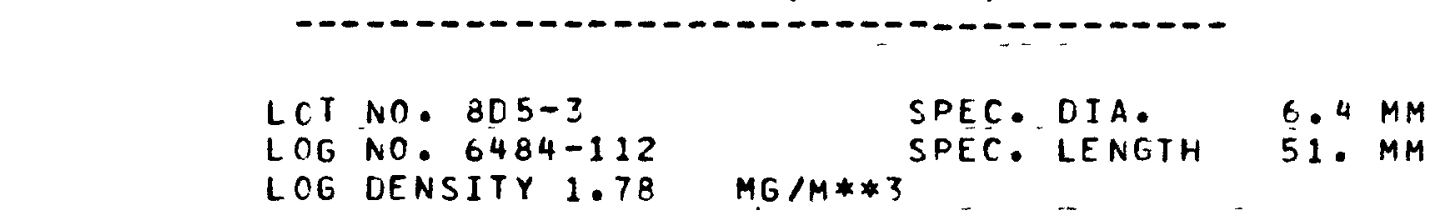

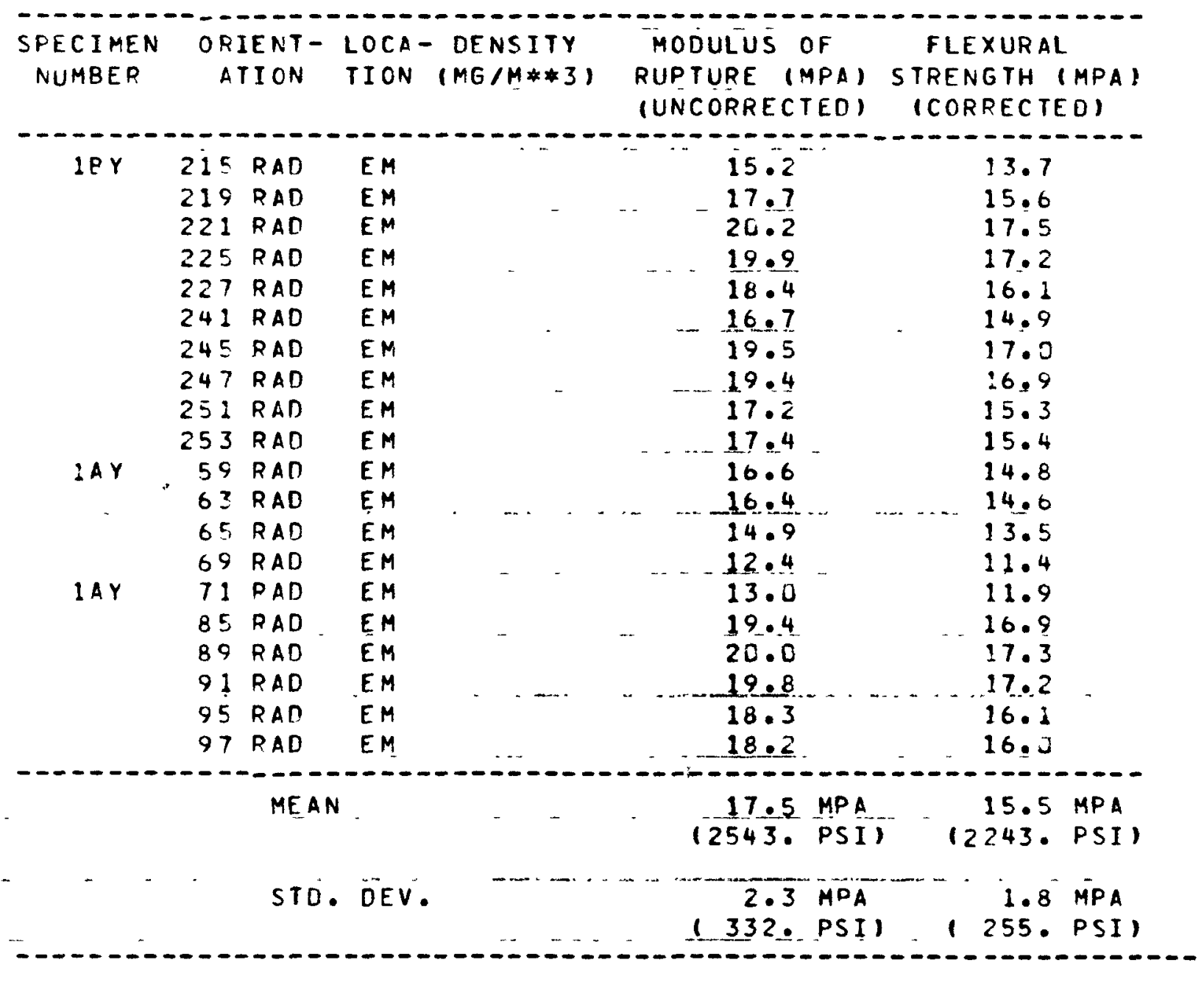


TABLE C-2 (Continued)

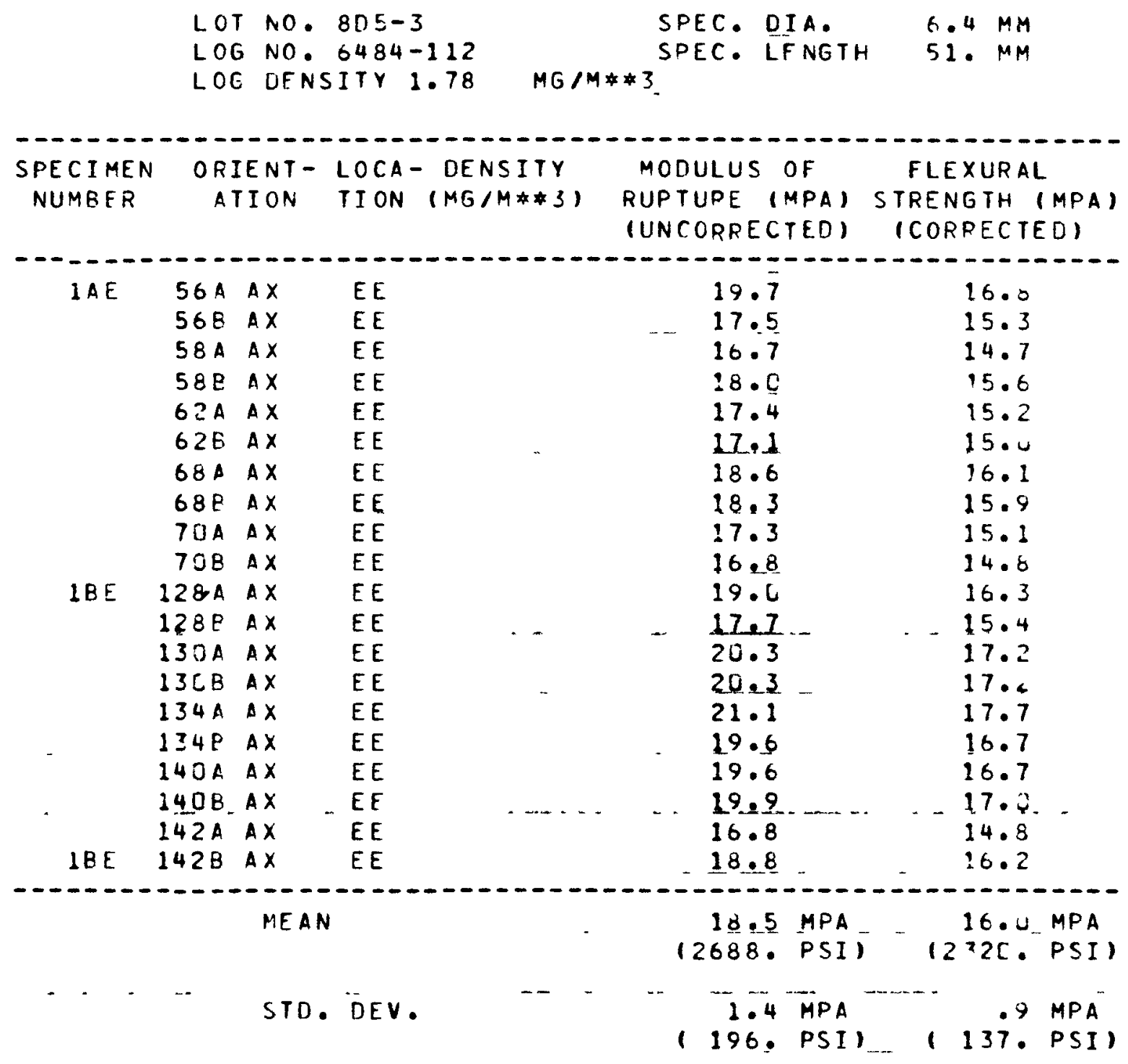

t)

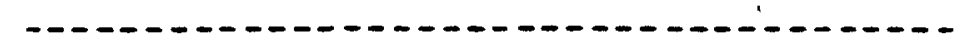

$\begin{array}{llll}\text { LOT NO. } 805-3 & \text { SPEC. DIA. } & 6.4 M M \\ \text { LOG NO. } 6484-112 & \text { SPEC. LFNGTH } & 51 . M M\end{array}$

LOE OENSITY 1.78 MG/M**3. 
TABLE C-2 (Continued)

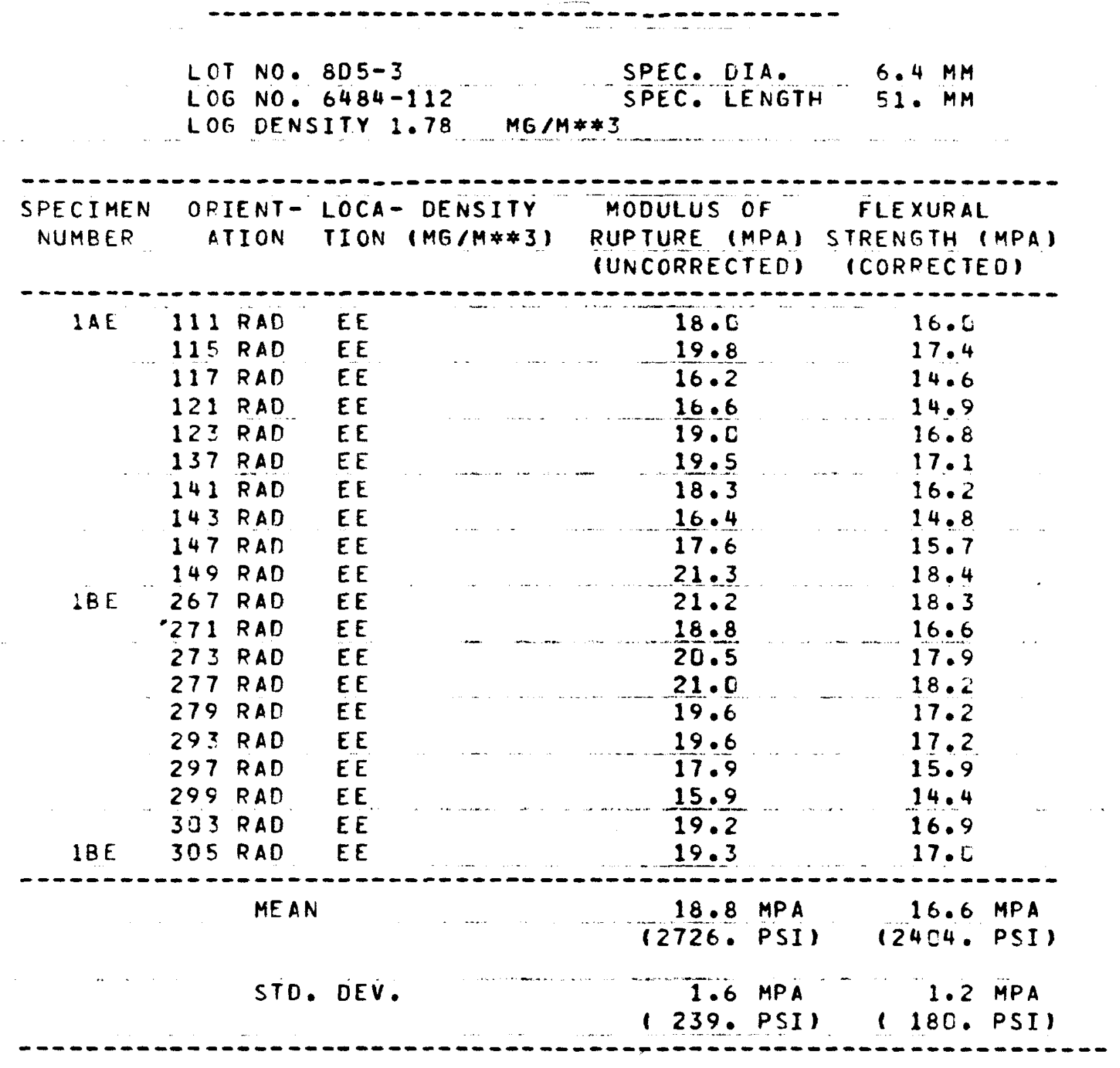


TABLE C-2 (Continued)

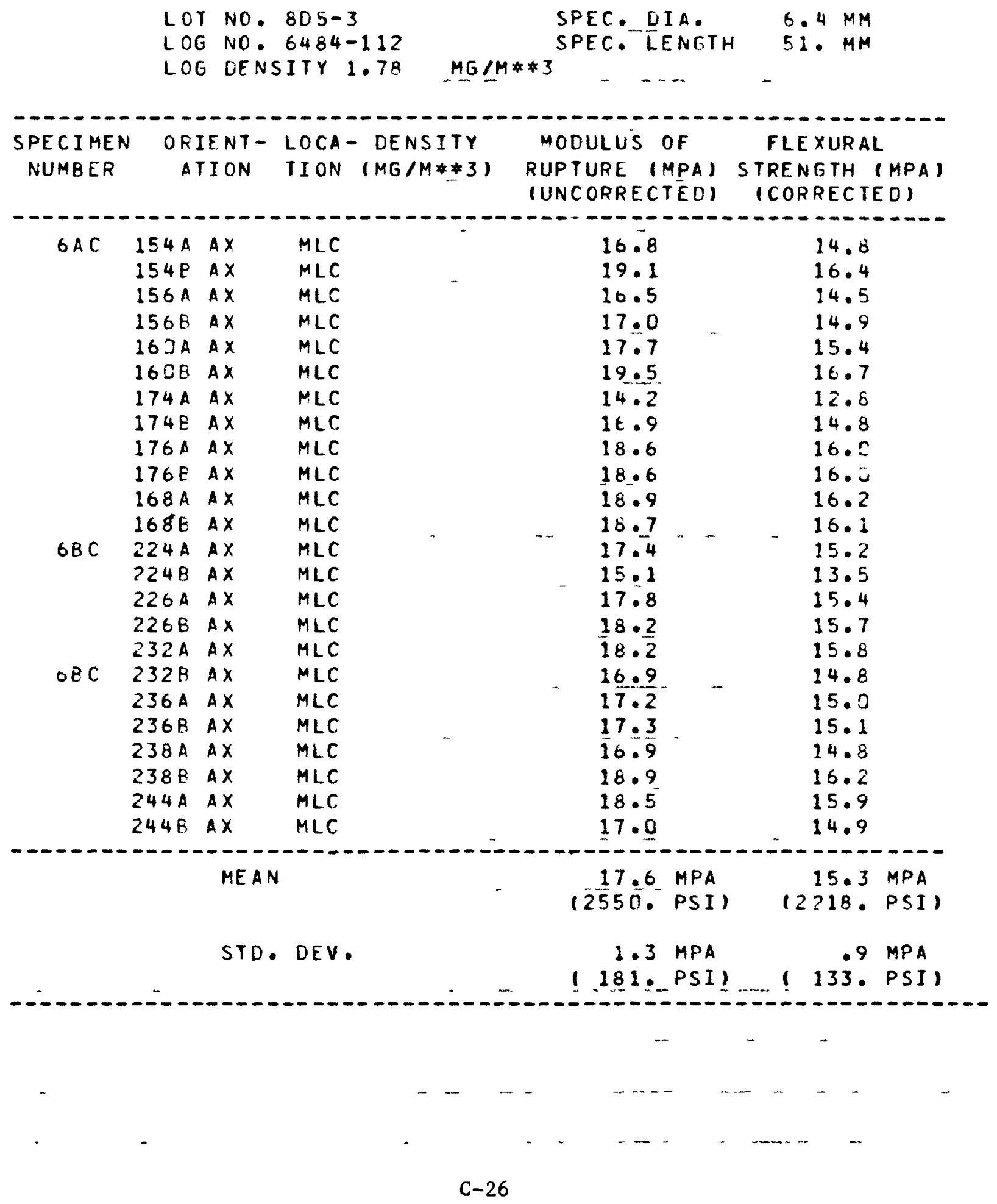


TABLE C-2 (Continued)

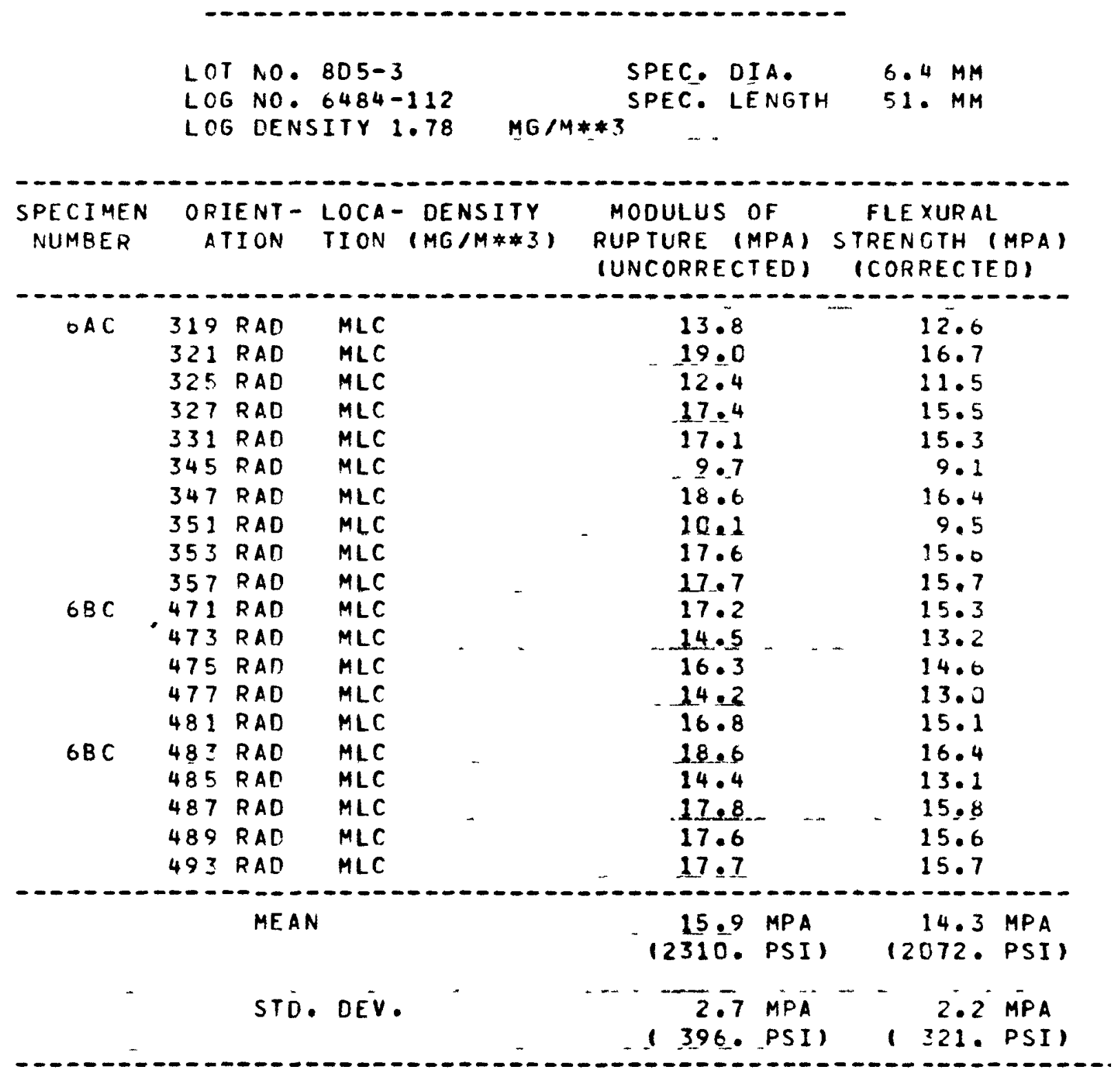


TABLE C-2 (Continued)

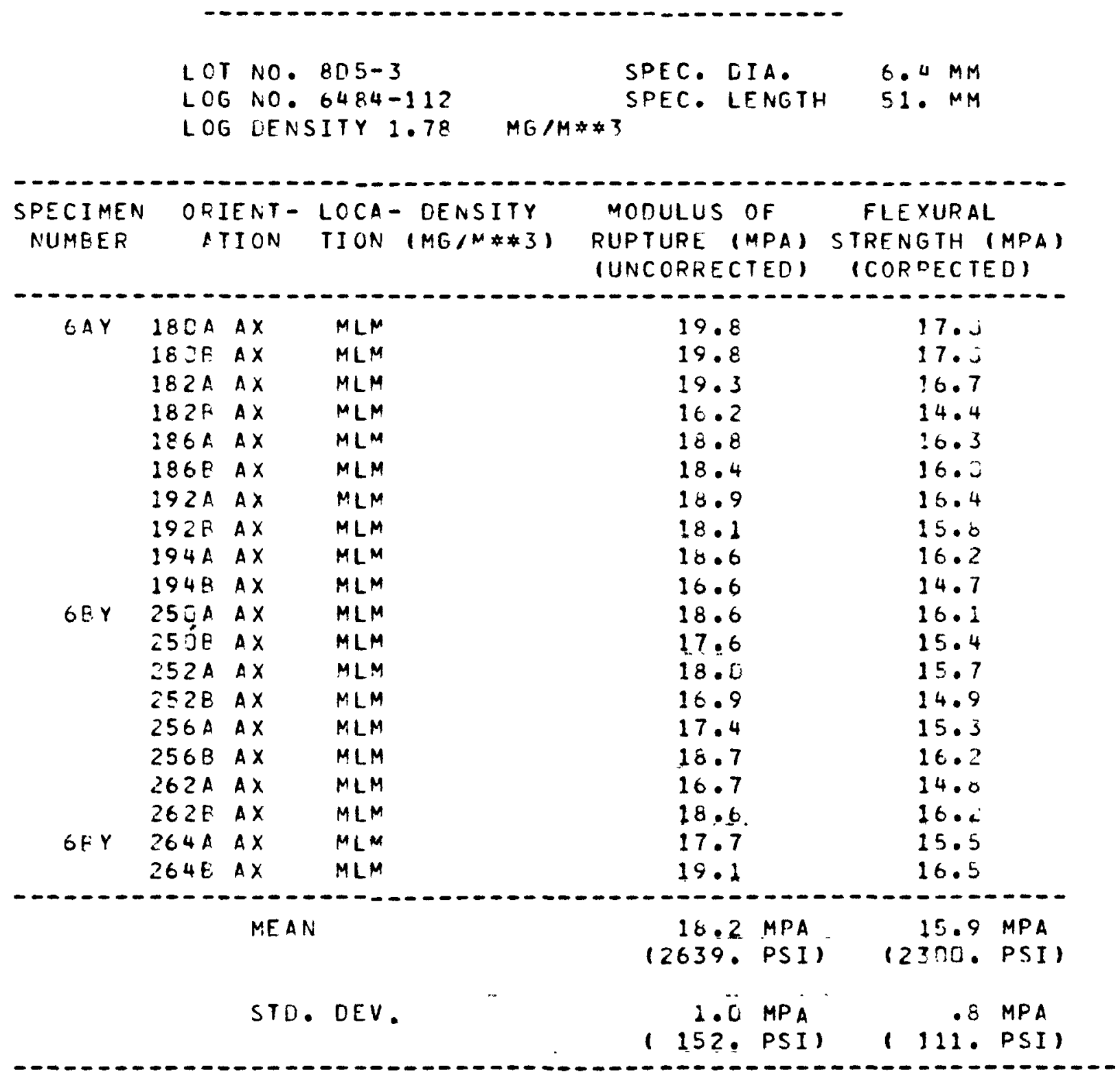


TABLE C-2 (Continued)

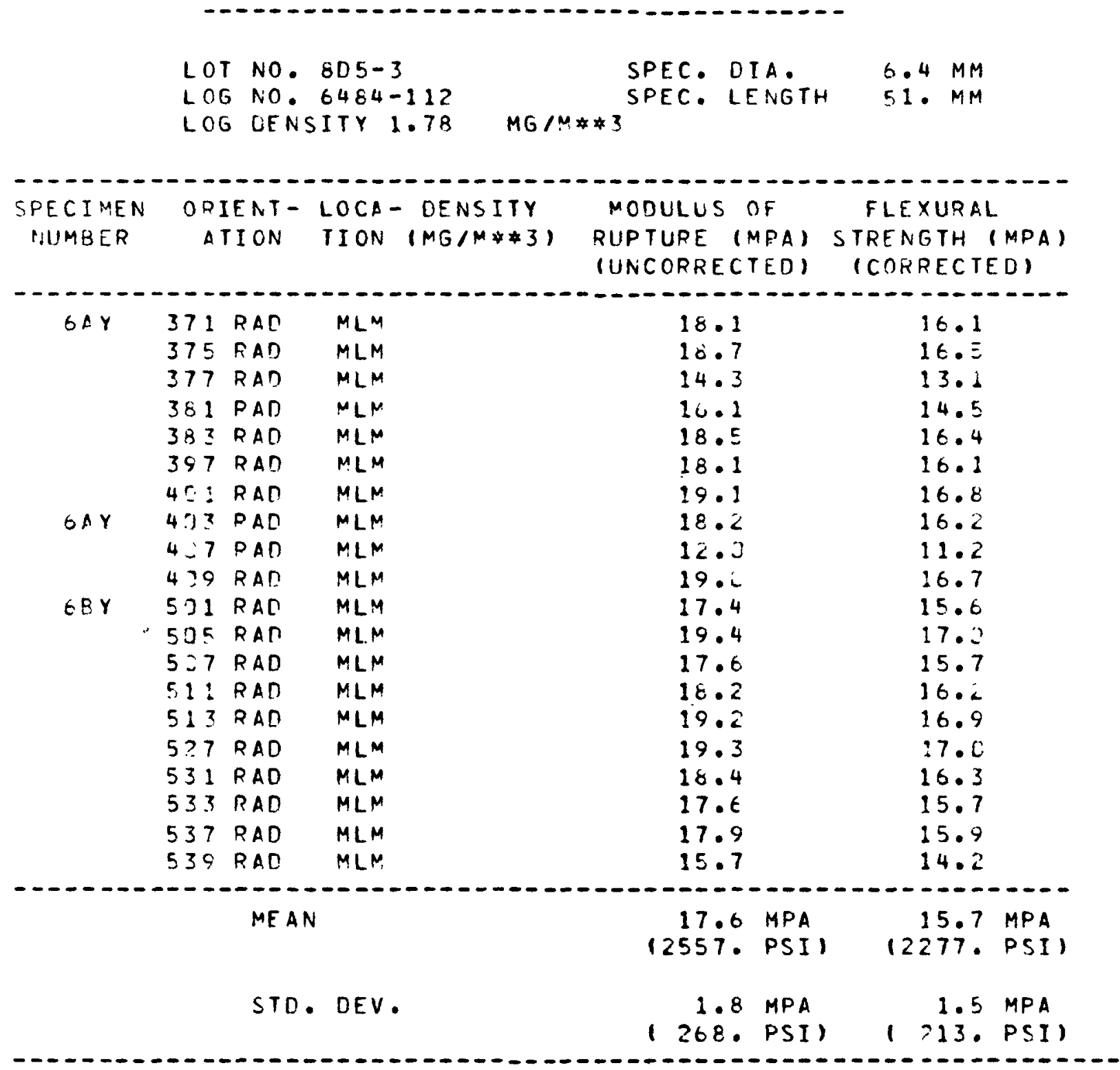


TABLE C-2 (Continued)

$\begin{array}{lll} & \text { LOT NO. 8R5-3 } \\ \text { LOC NO. 6484-11? SPEC. DIA. } & \text { SPEC. LEIVGTH MM } \\ \text { LOG LENSITY } 1.78 & \text { MG/M\# MM }\end{array}$

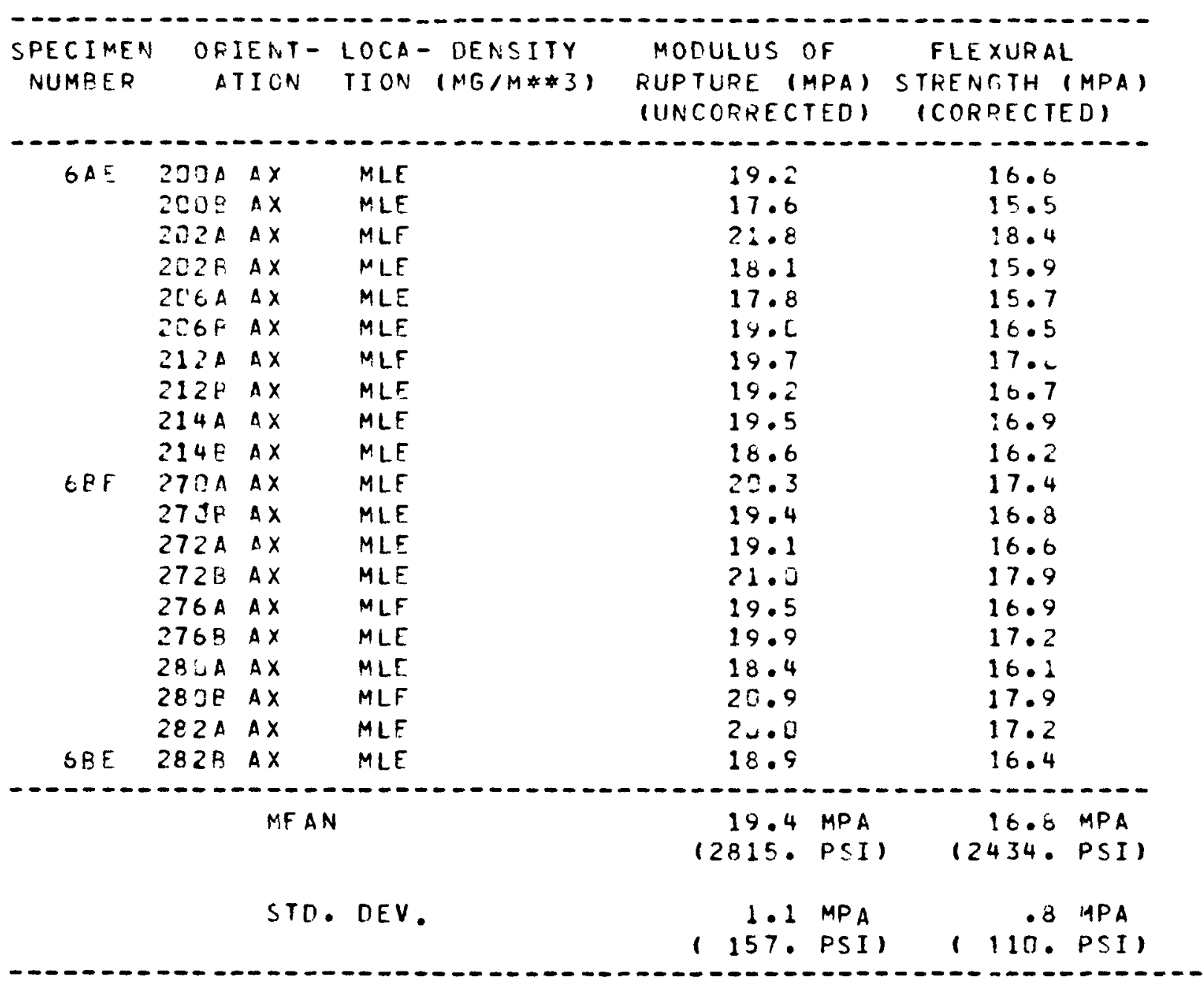


TABLE C-2 (Continued)

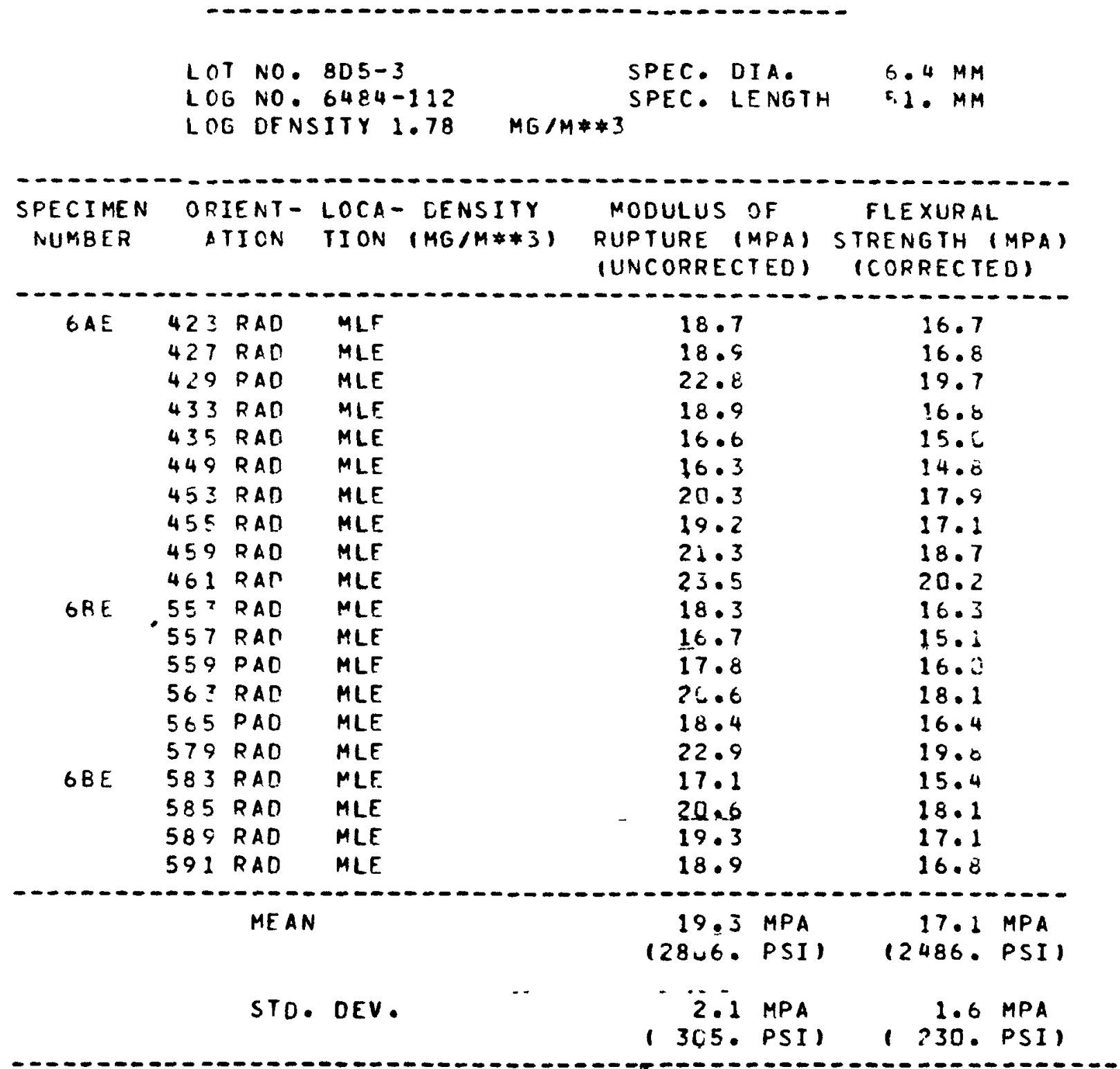


TABLE C-2 (Continued)

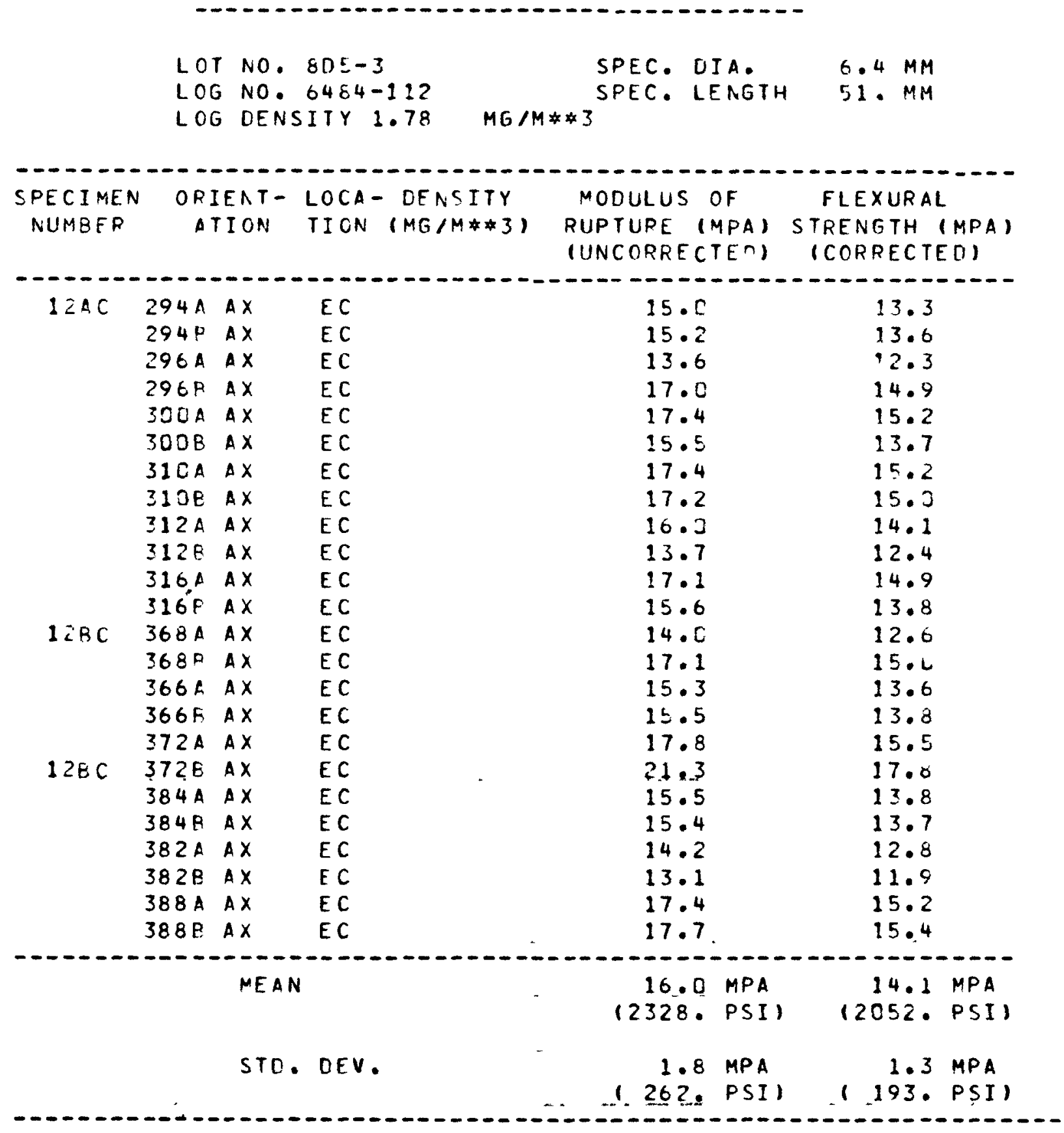


TABLE C-2 (Continued)

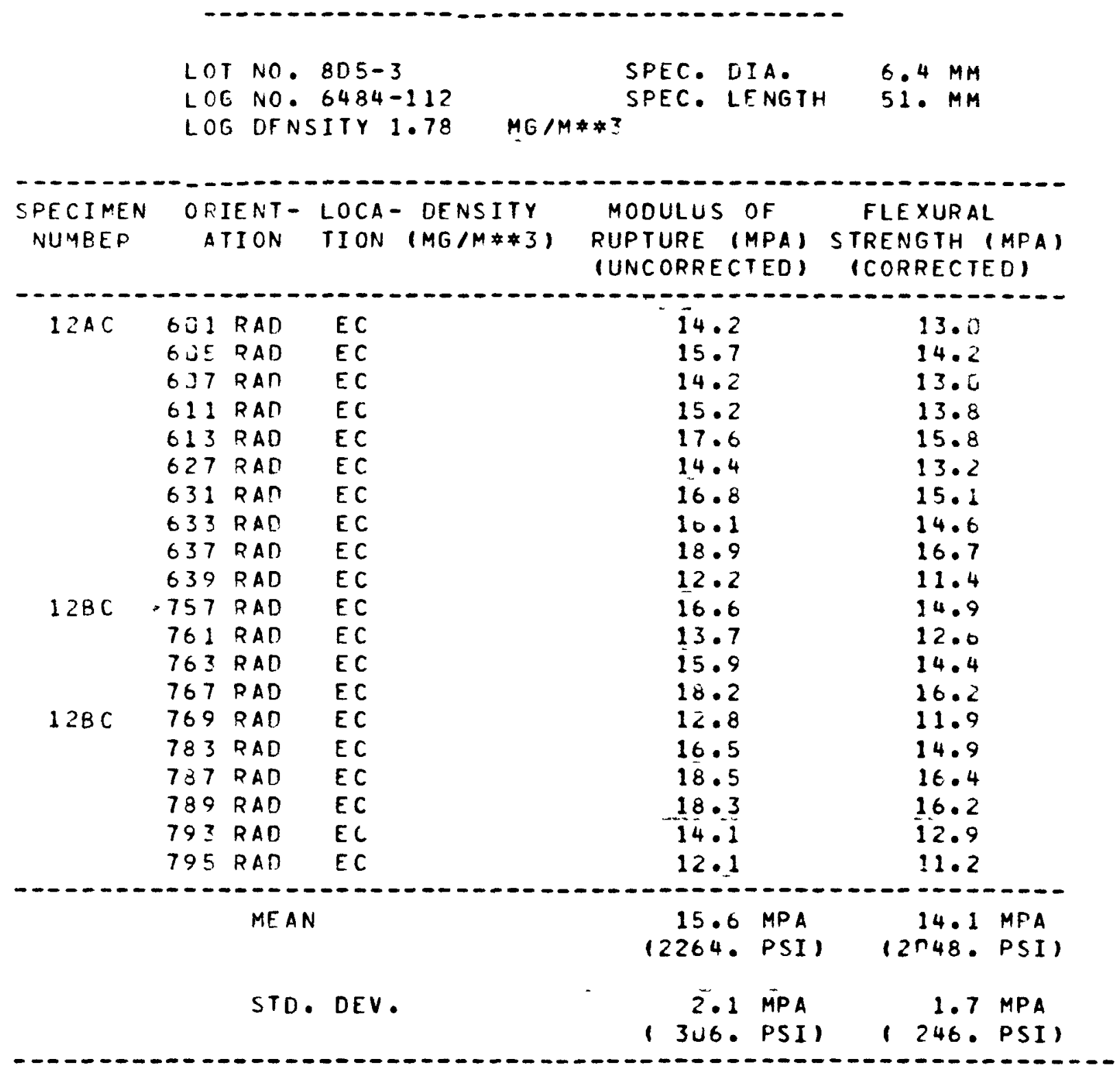


TABLE C-2 (Continued)

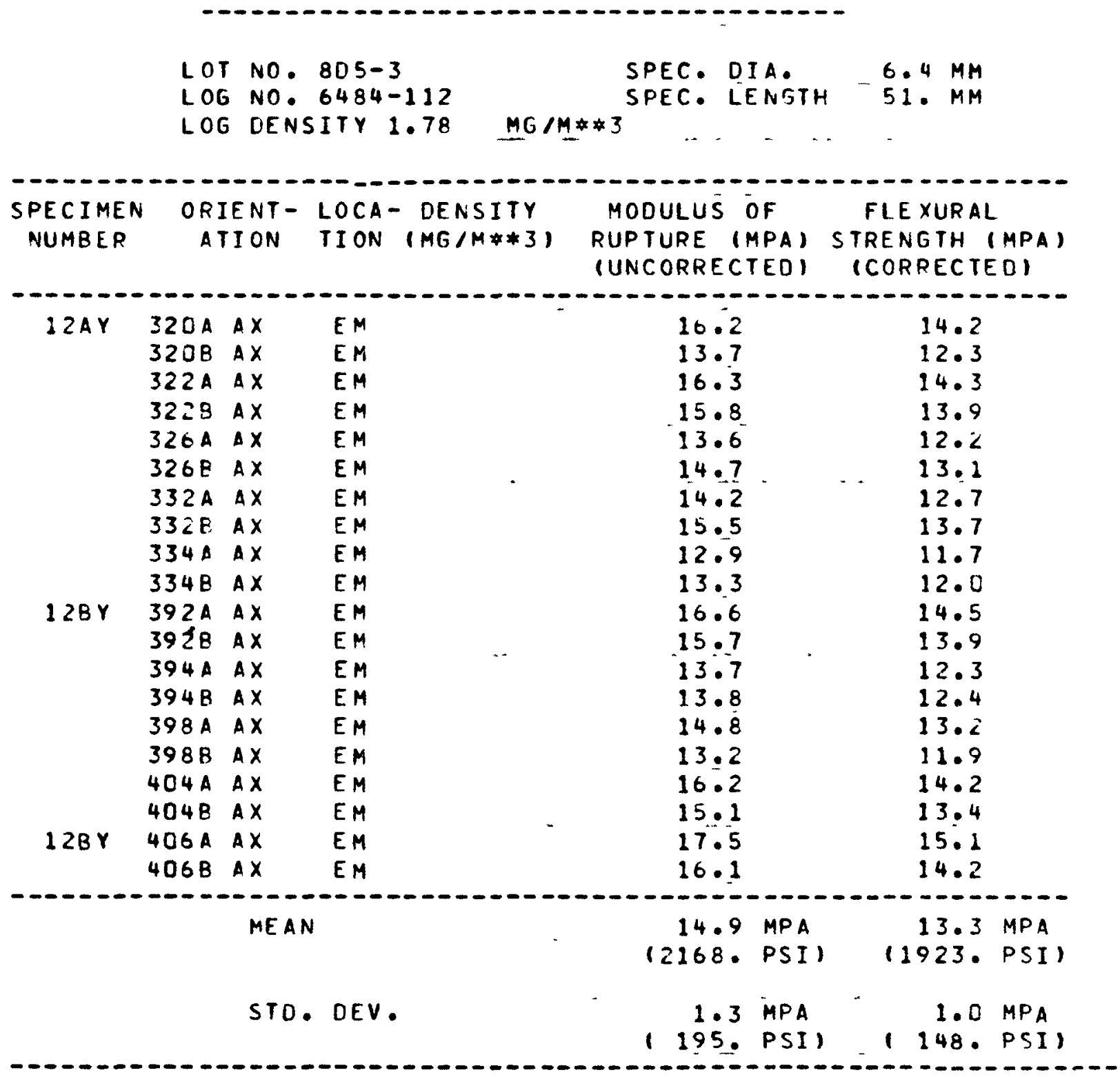


TABLE C-2 (Continued)

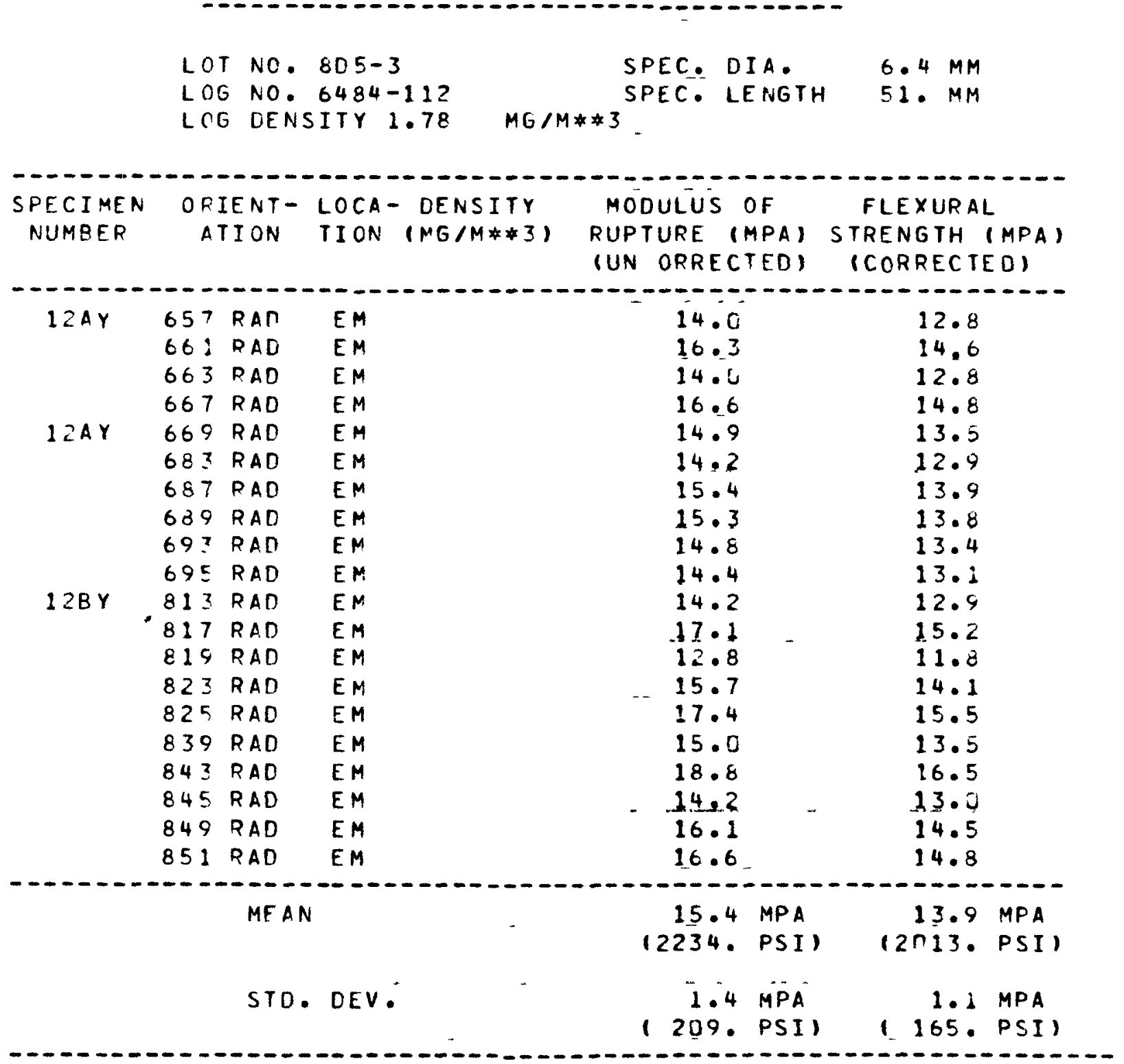


TABLE C-2 (Continued)

LOT NO. $805-3$
LOG NO. 6484-112 SPEC. DIA.
LOG LENSITY $1.78 \quad$ SPEC. LENGTH

SPECIMEN ORIENT- LOCA DENSITY MODULUS OF I FLEXURAL
NUMBER ATION TION (MGIM\#*3) RUPTURE (MPA) STRENGTH (MPA)
(UNCORRECTED) (CORRECTED)

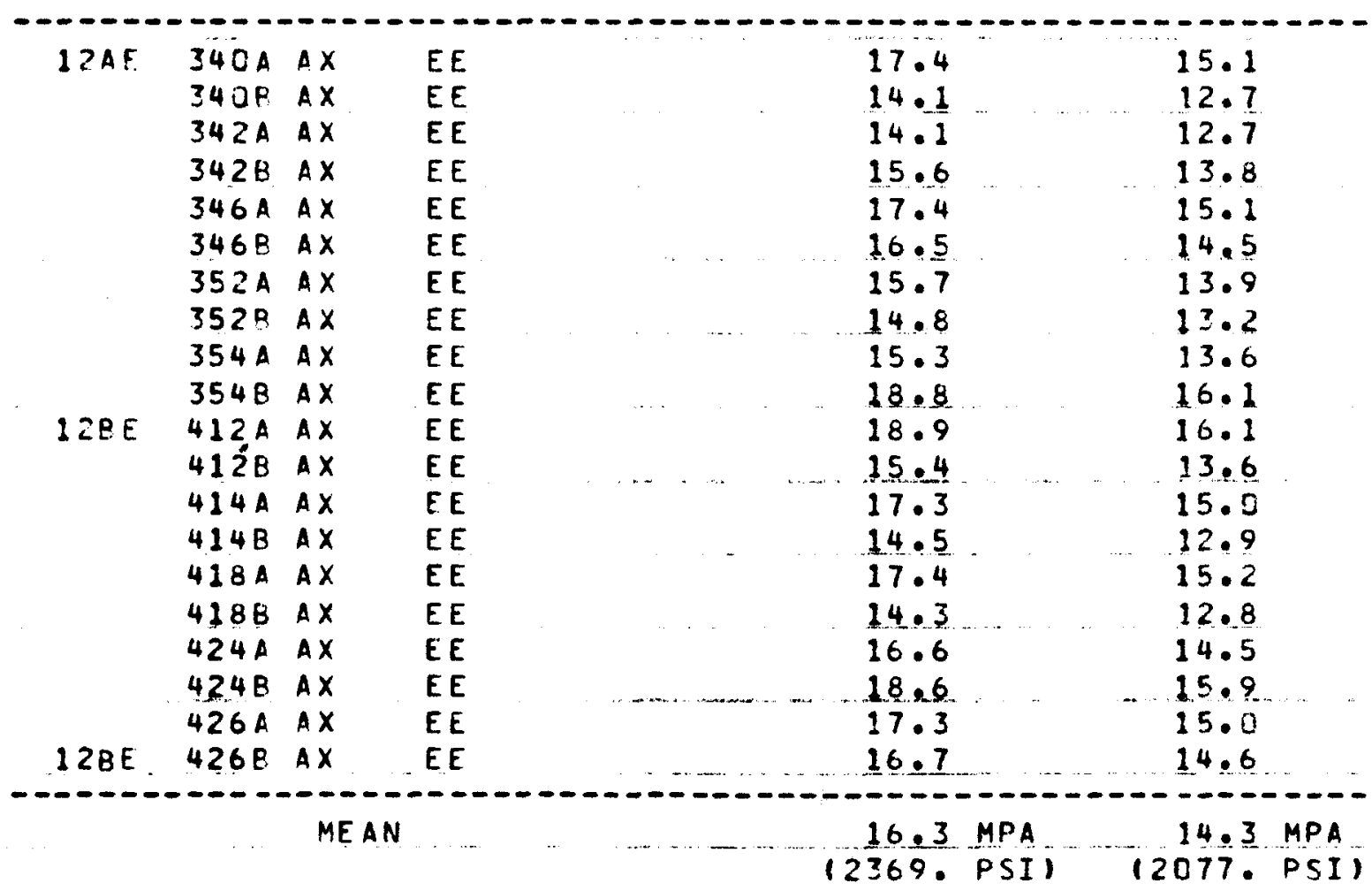

$\begin{array}{ccc}\text { STO. DEV. } & 1.5 \text { MPA } & 1.21 \mathrm{MPA}\end{array}$


TABLE C-2 (Continued)

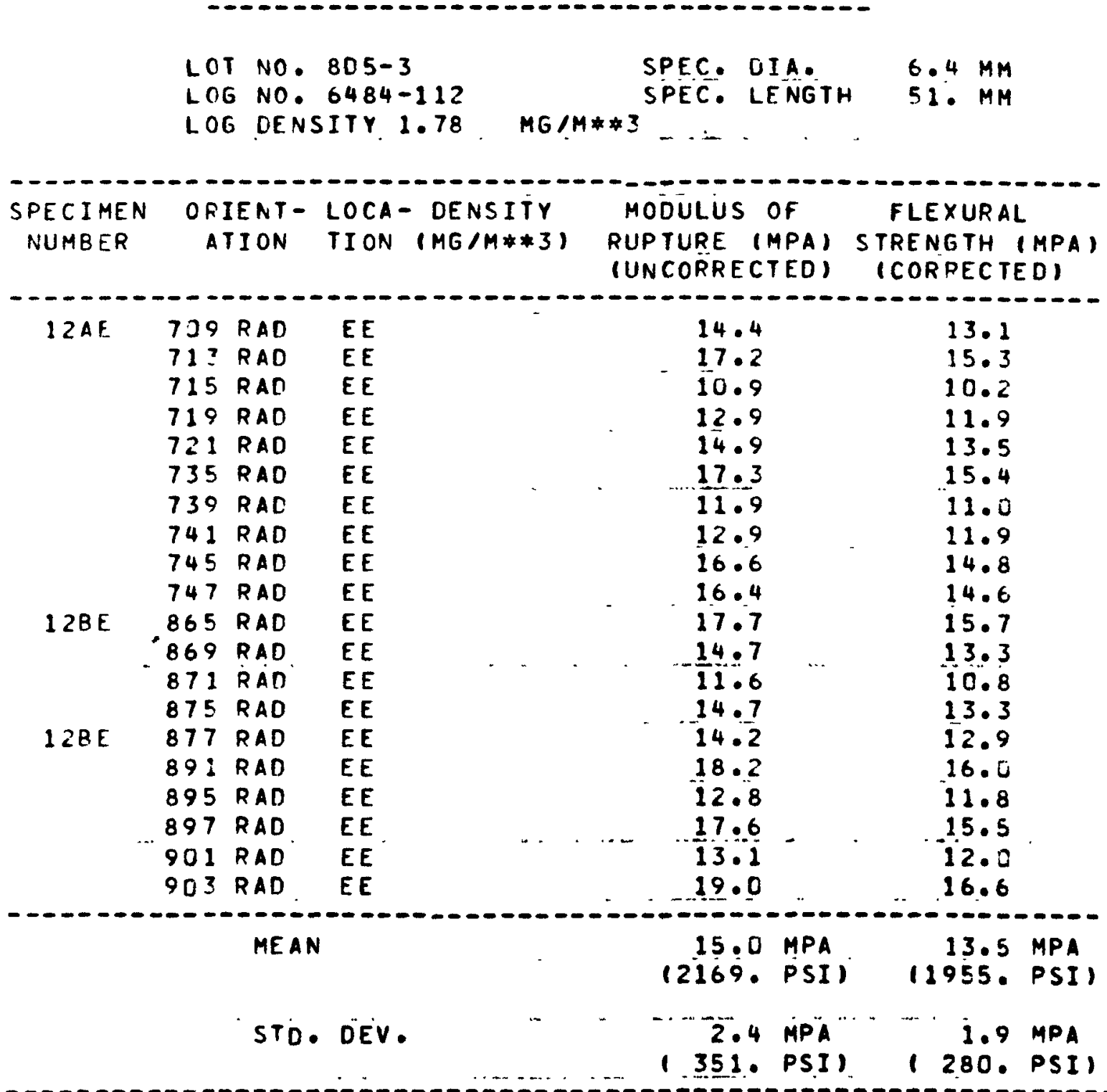


TABLE $\mathrm{C}-3$

COMPRESSIVE PROPERTIES OF PGX GRAPHITE

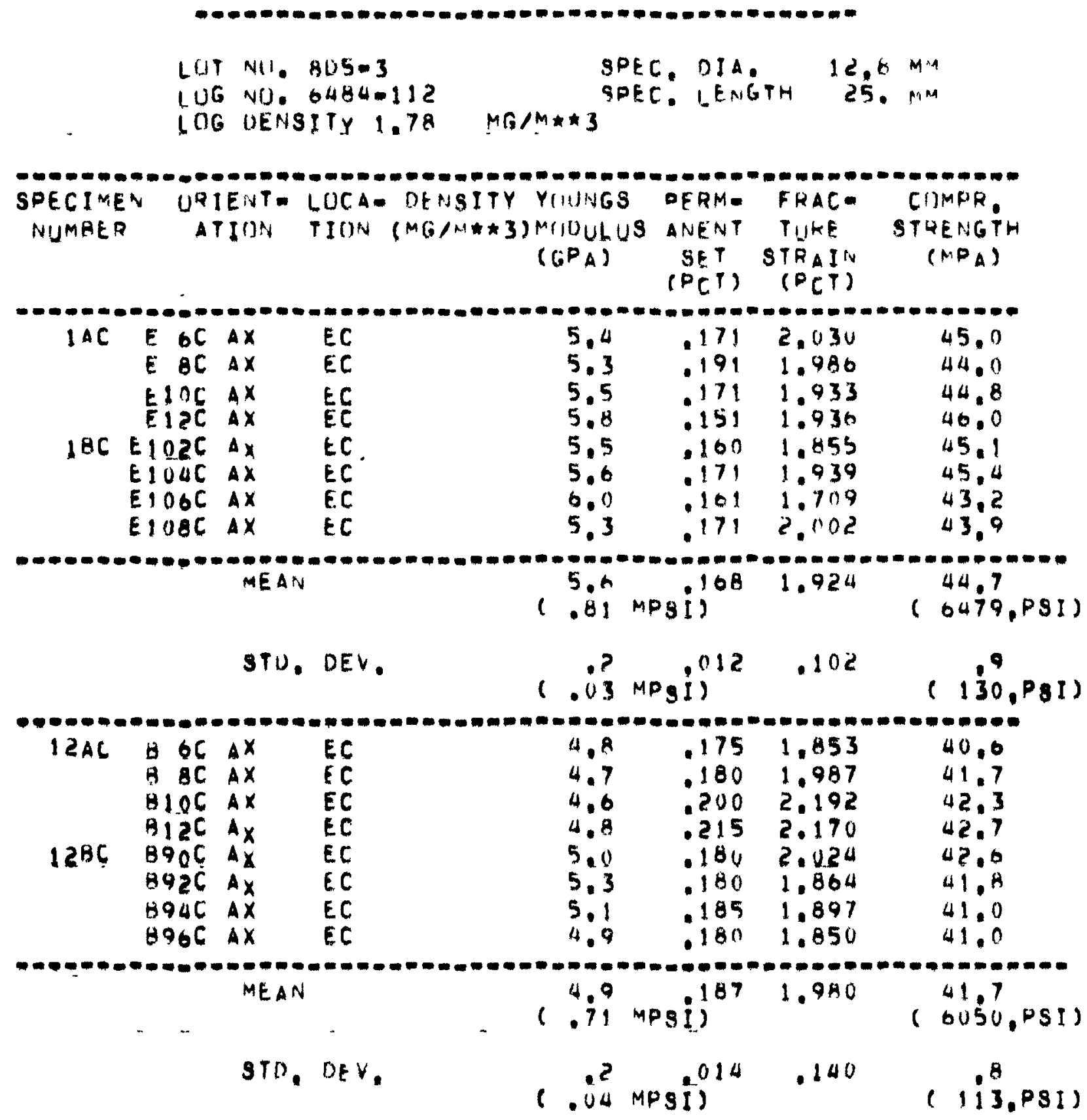

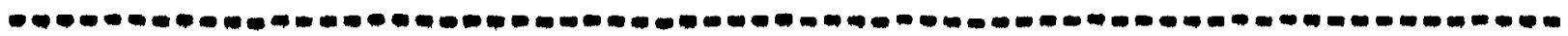


TABLE C-3 (Continued)

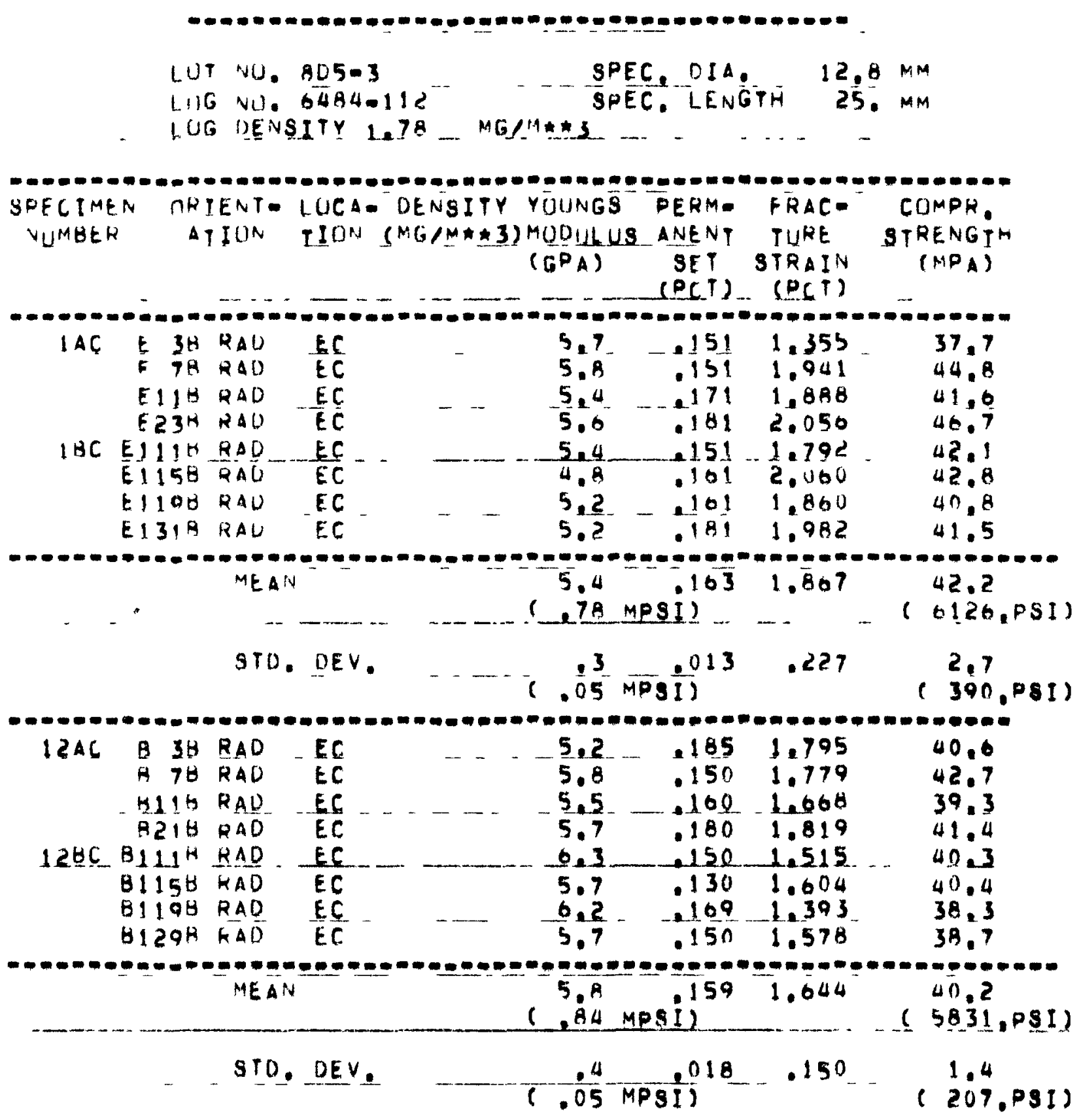


TABLE C-3 (Continued)

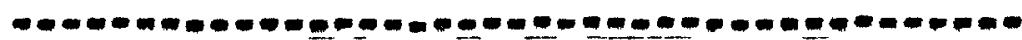
LUT NO, BDHE 3
SPEC. DIA,
$12.8 M M$
LUG NU. 6484-112
SPEC. LENGTH 25. MM

LOG DENSITY_. 78 MGLME

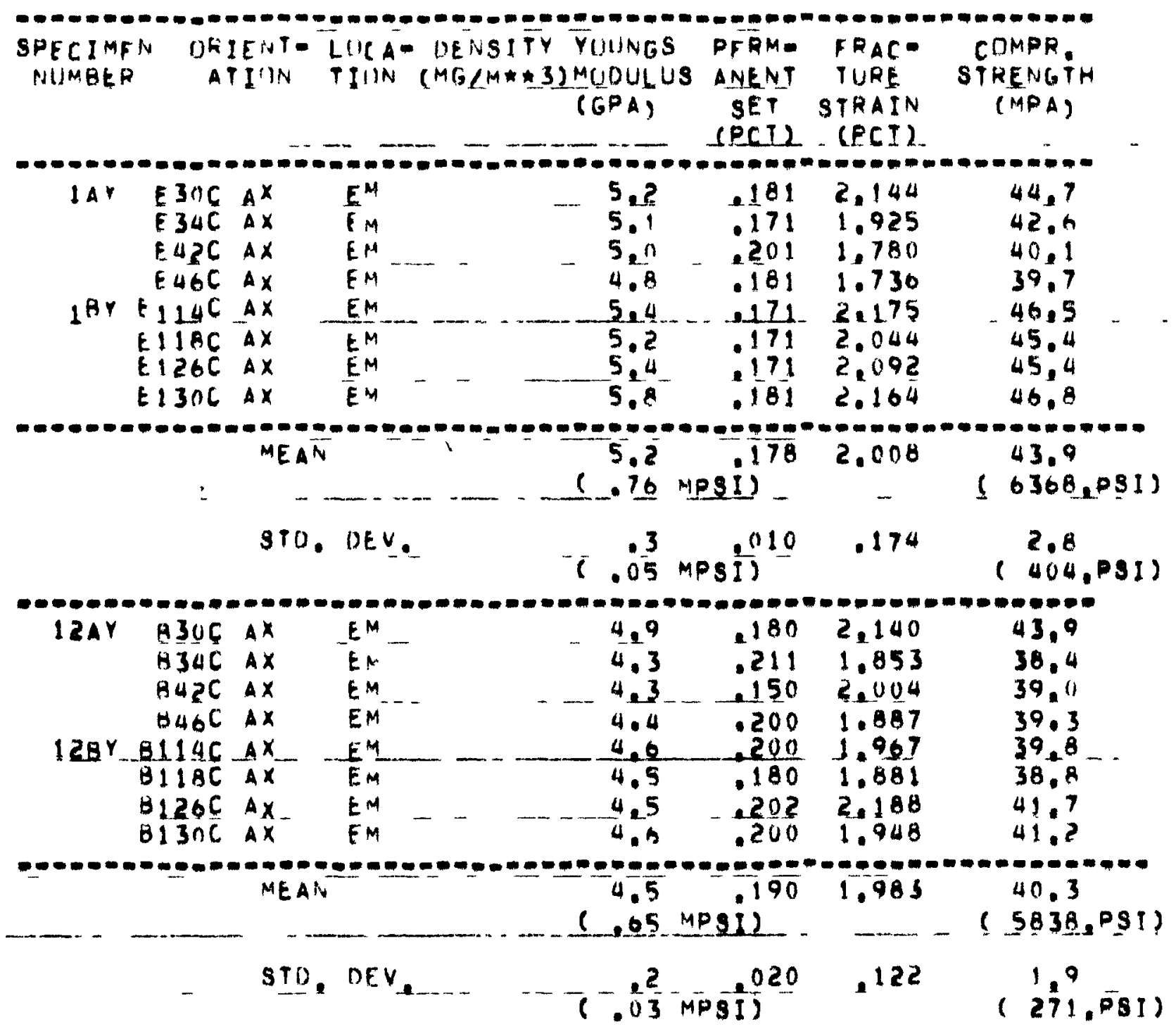

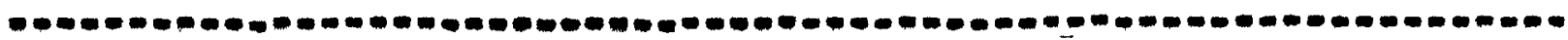


TABLE C-3 (Continued)

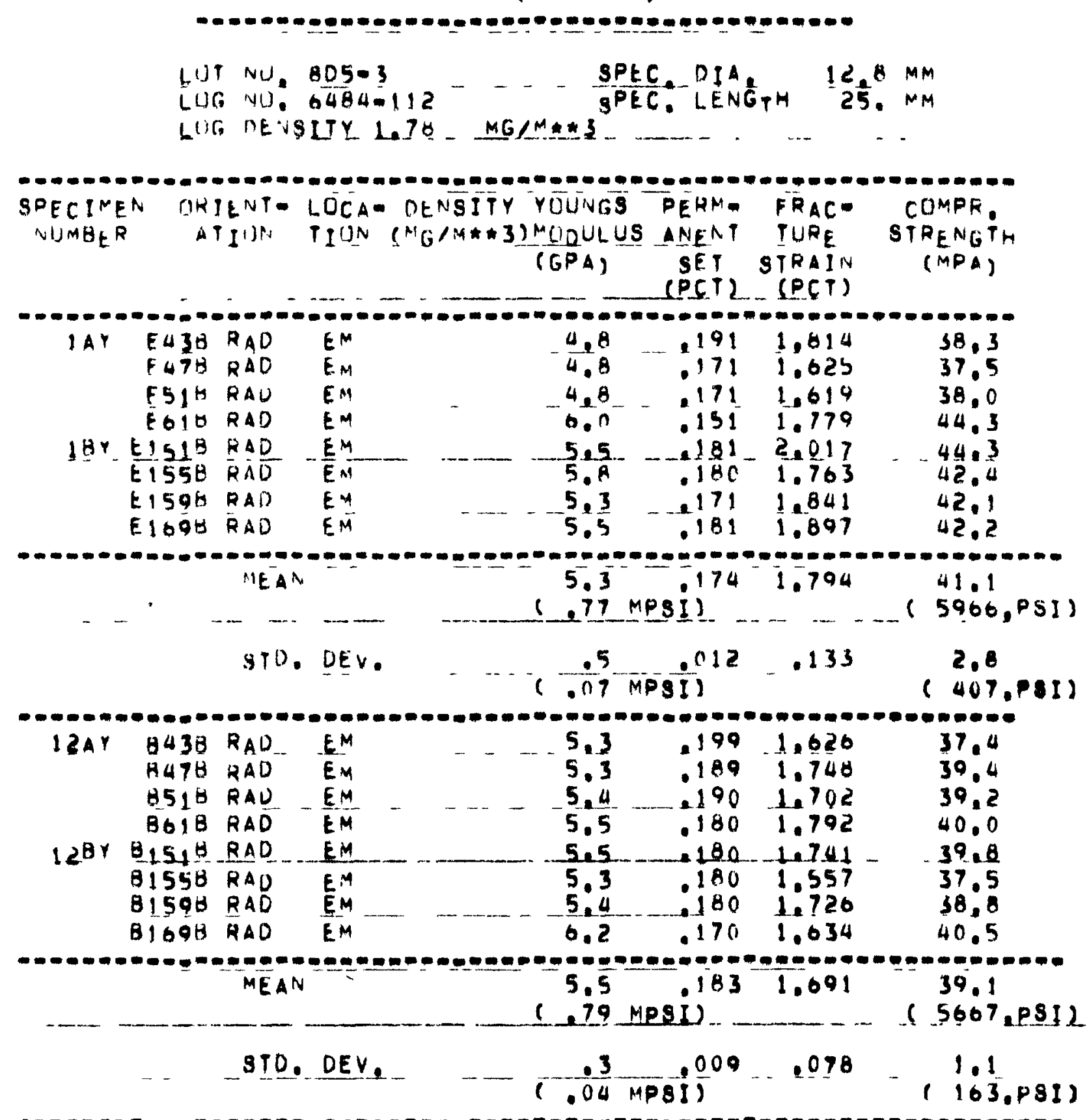


TABLE C-3 (Continued)

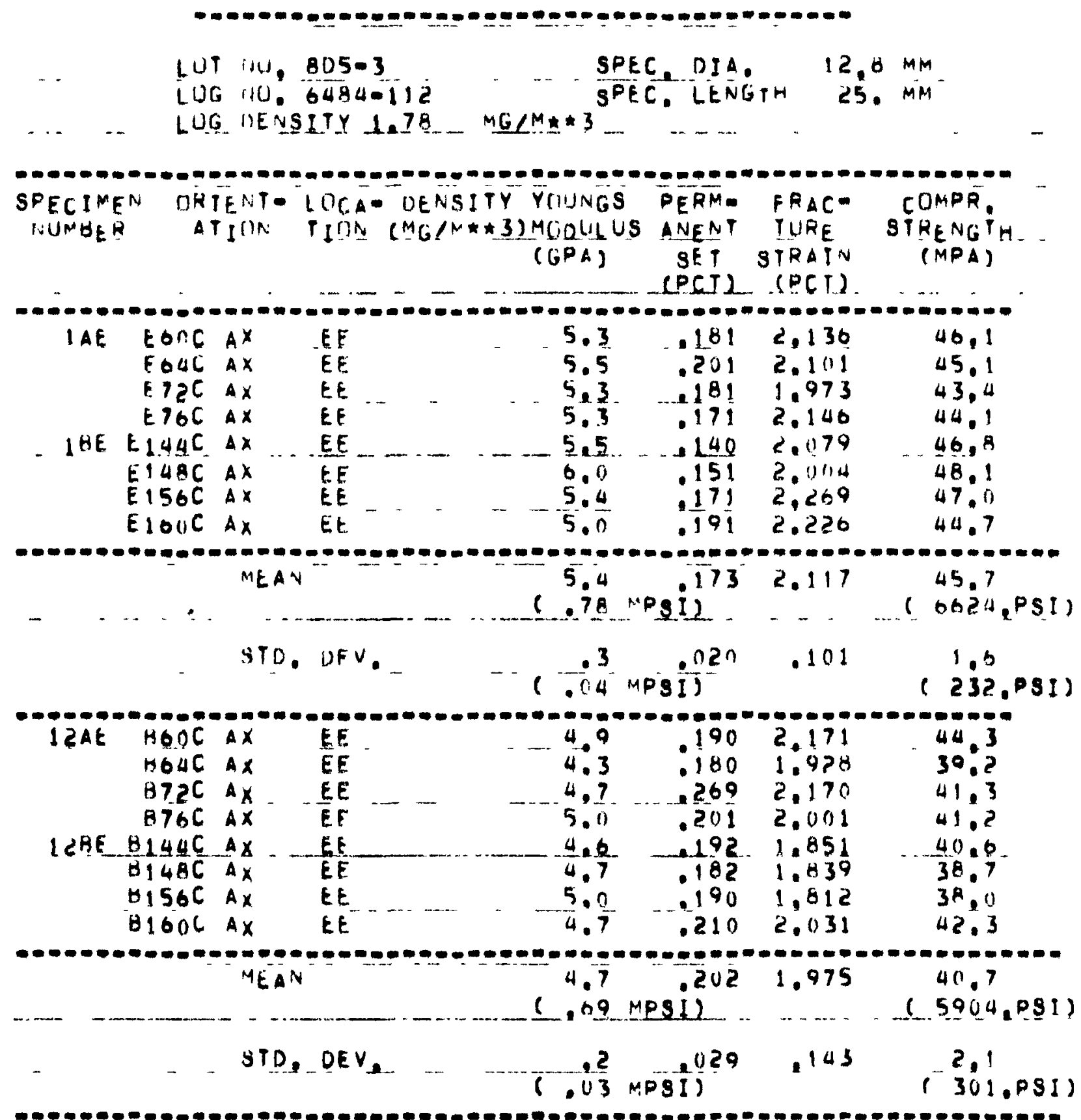


TABLE C-3 (Continued)

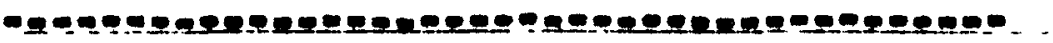

LUT NO. ADS-3

LDG ND. $6484-112$ SPEC, DIS. $12,8 \mathrm{~mm}$ SPEC. LENGTH $25 . \mathrm{Mm}$

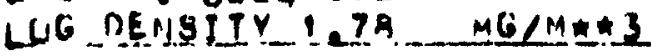

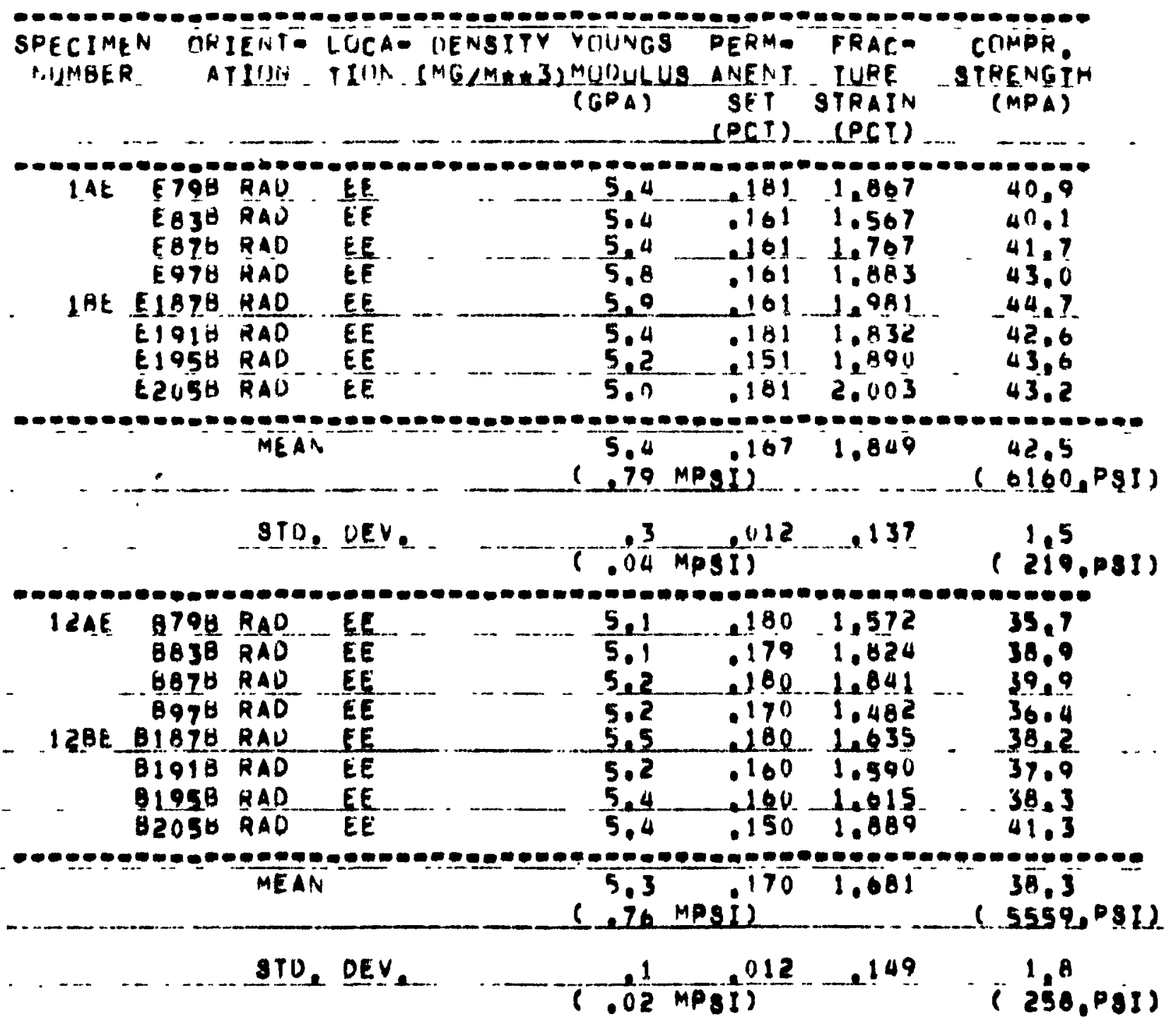

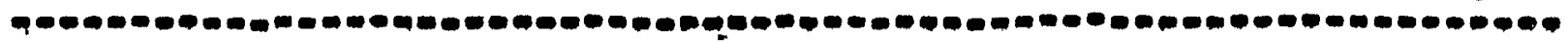


TABLE C-3 (Continued)

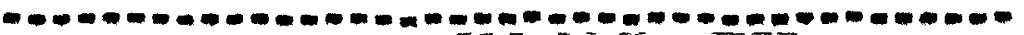

IUT NII. AUSE-3

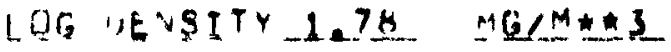

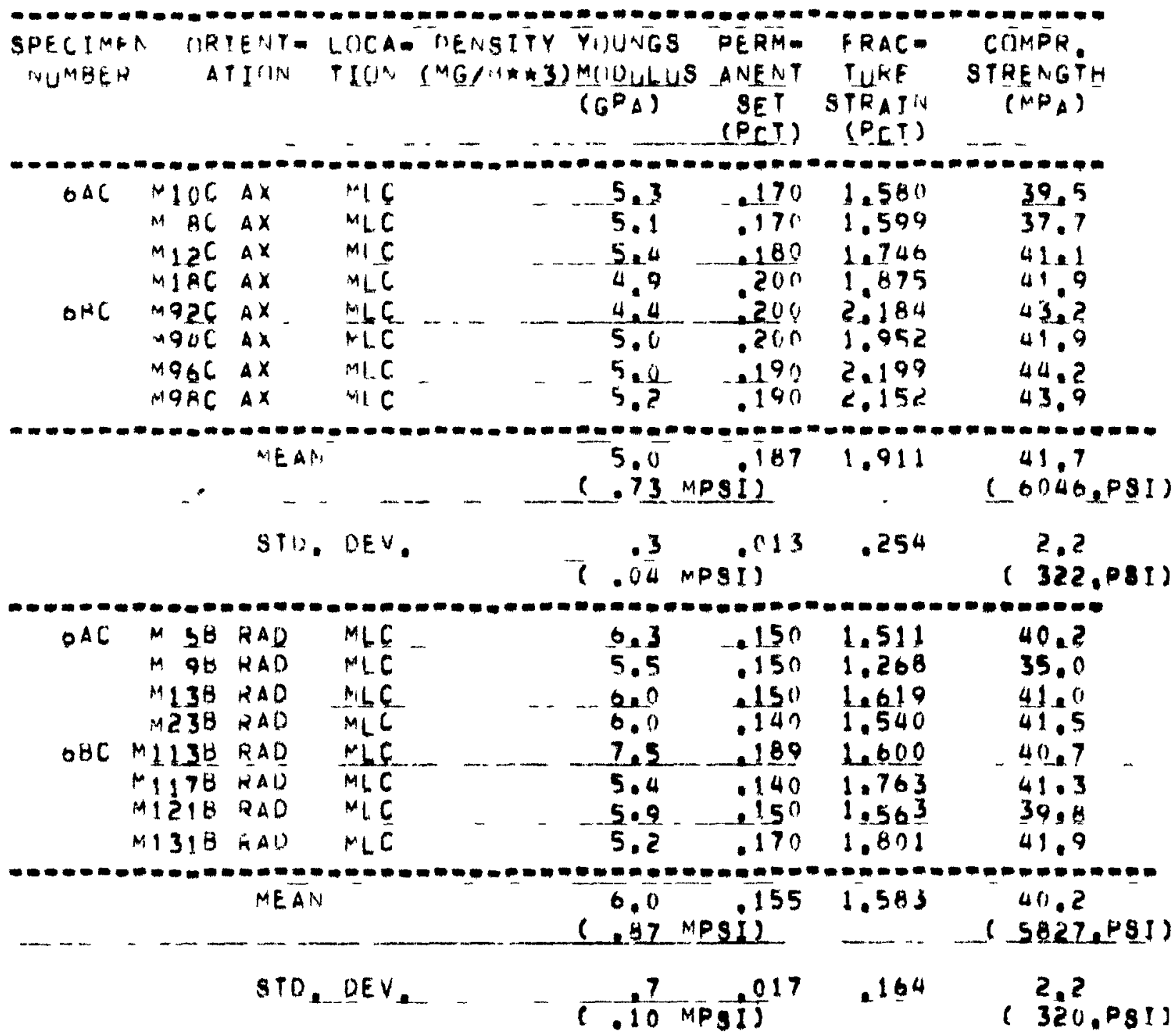

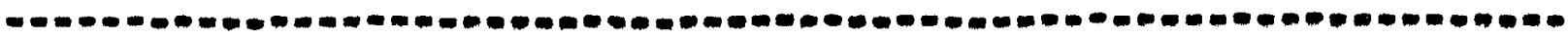


TABLE C-3 (Continued)

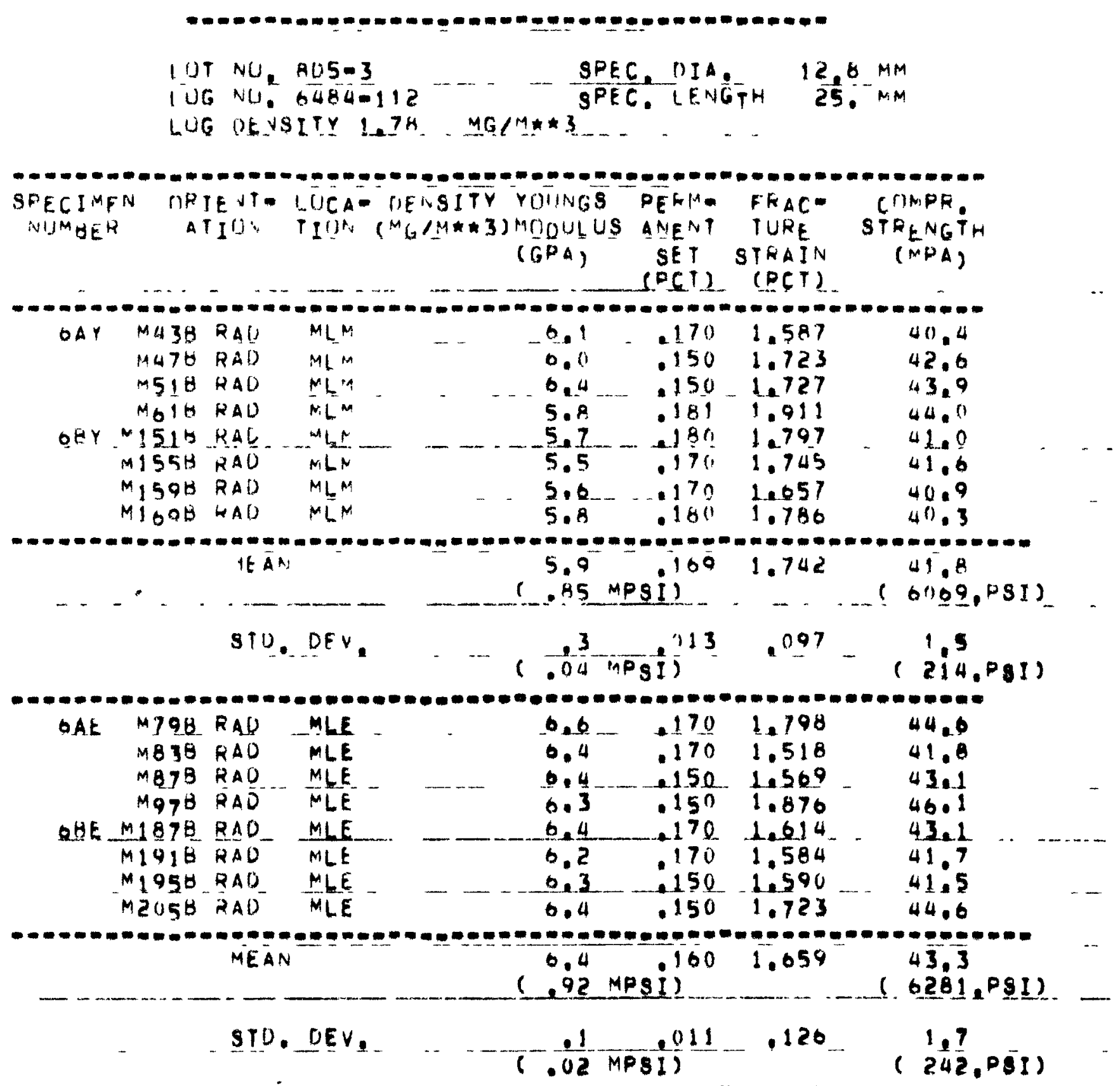


TABLE C-3 (Continued)

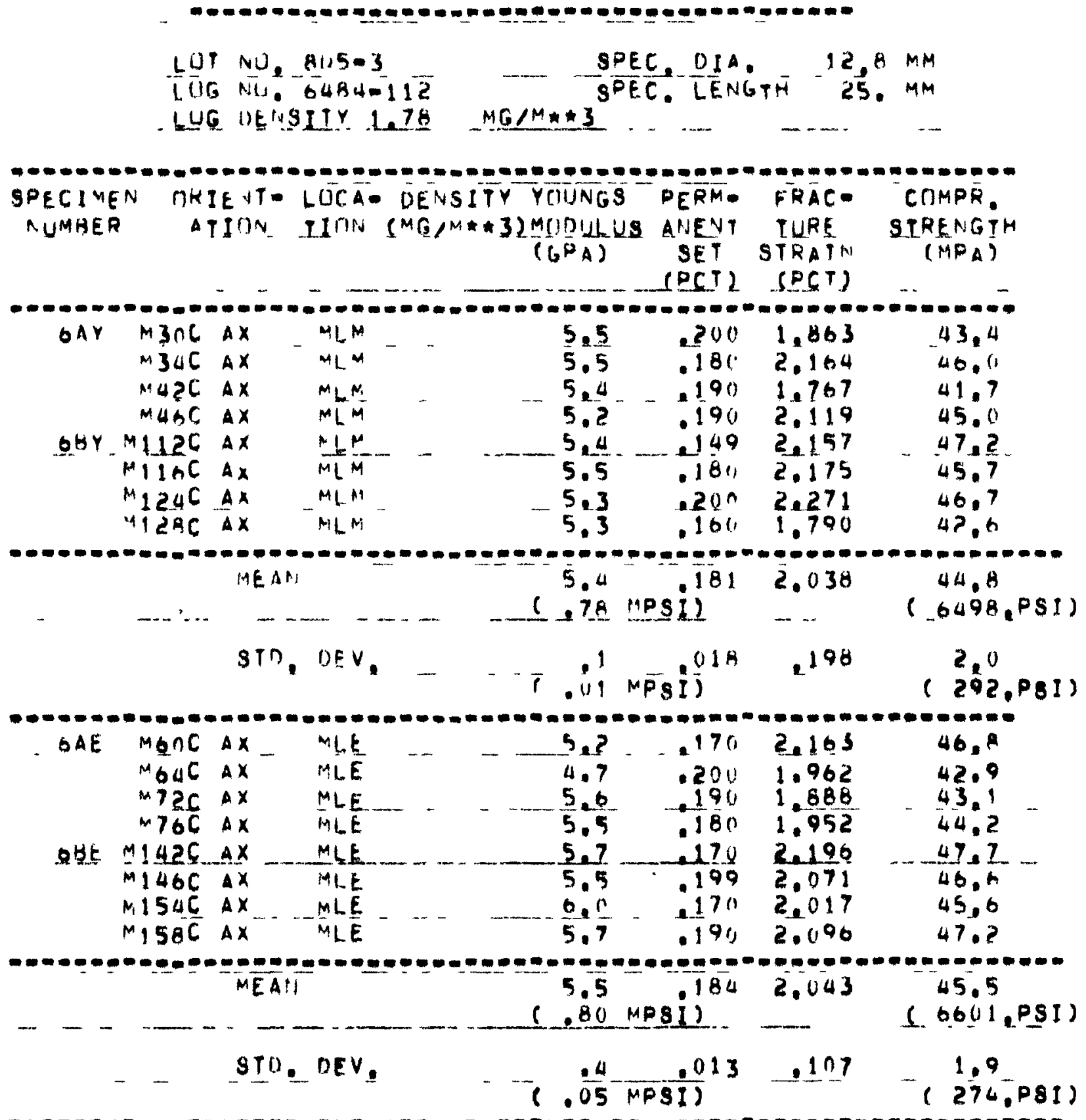



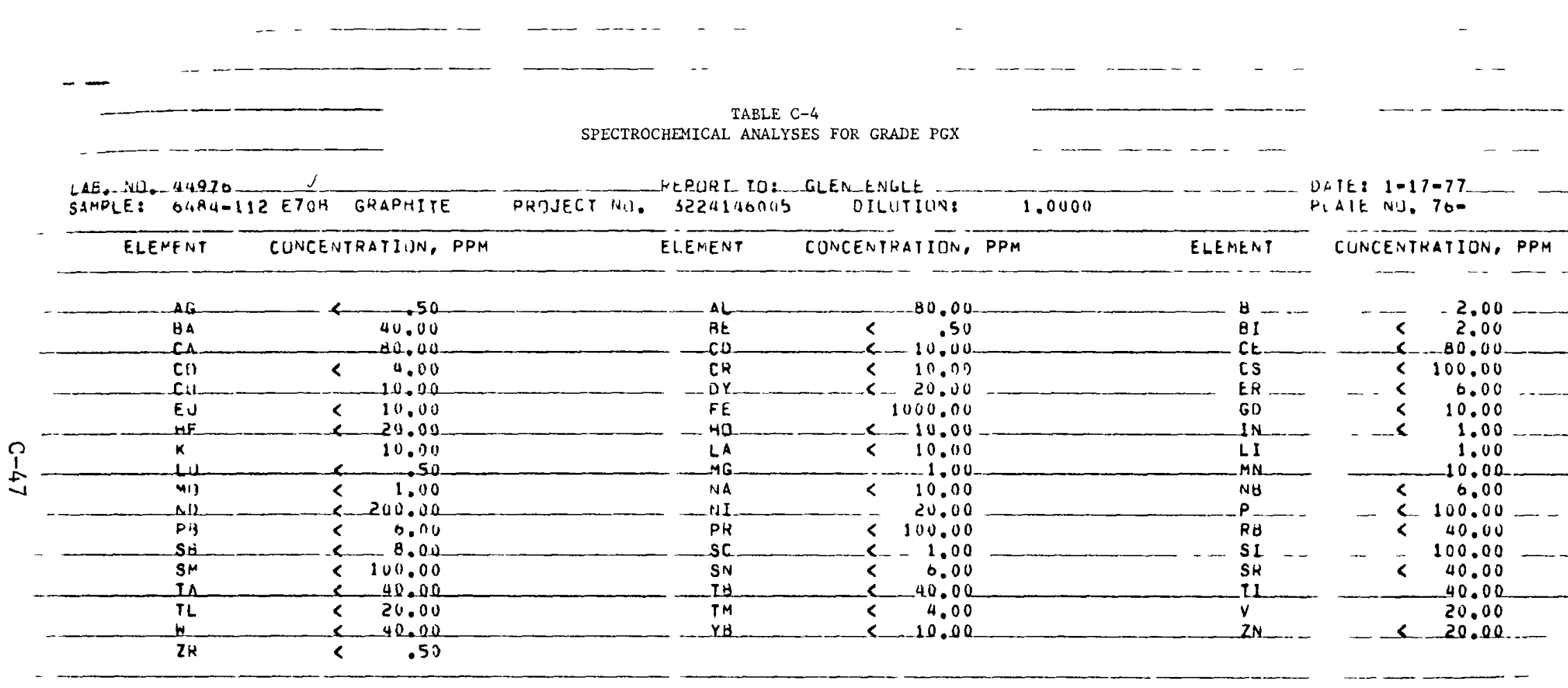

CDNCENTRAIION BASED ON CRGINAL SAMPLE BEFORE DILUTI JN WIIH_DLUENL

> MEANS GRE $T$ TER THAN

C MEANS LESS THAN IHE SENSIIIVITYOF THE SPECIROGRAPMIC PROCEUURE USED

RESULTS AHE CORRECT WITHIN A FACTOR IF $40 \%$, (UNE STANDARO DEVIATION) 
TABLE C-4 (Continued)

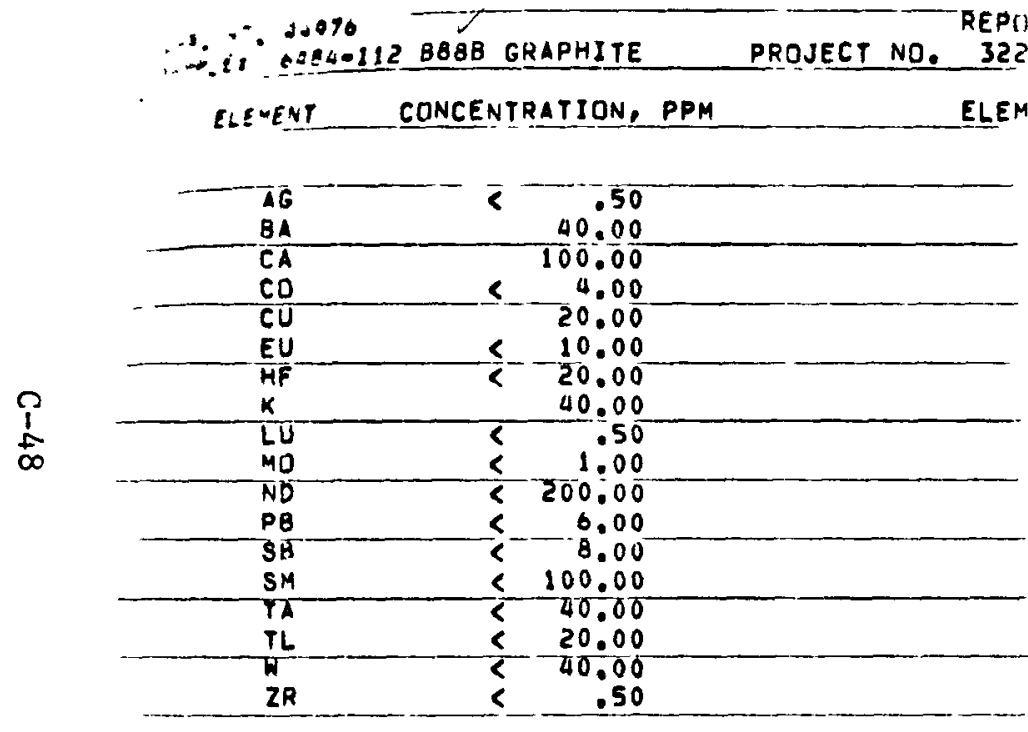

3224146005 GLEN ENGLE

$+\cdots+\cdots$

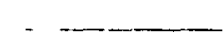

LEMENT CONCENTRATION, PPM PLAIt $1-17-79$

COACENTRATION, PPM
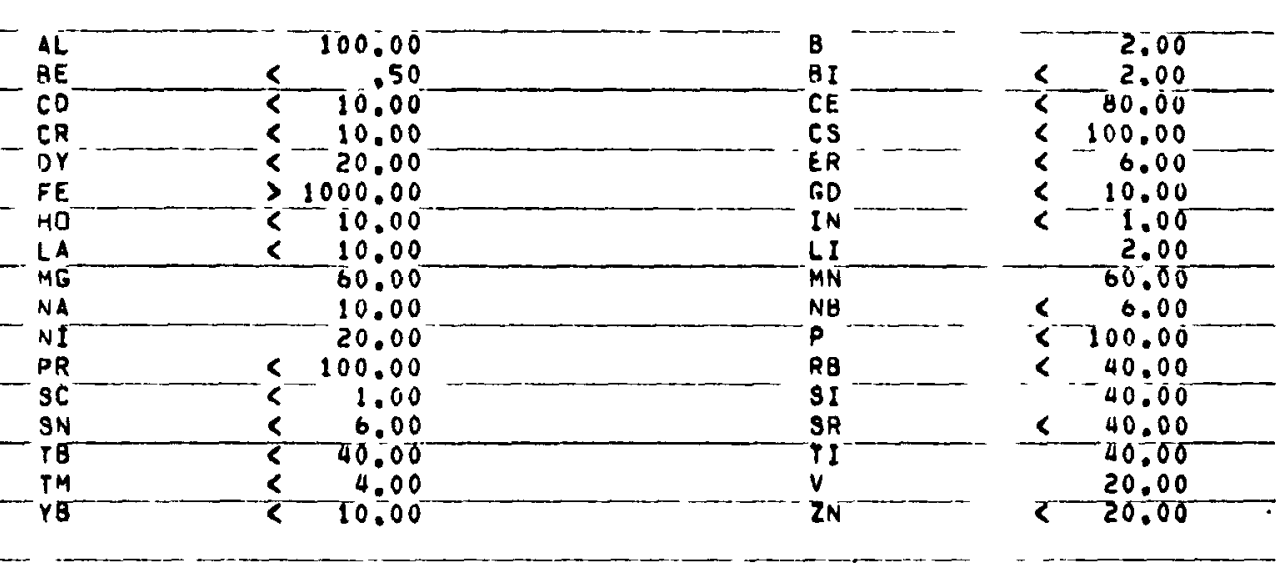

CONCENTRATION BASED ON ORGINAL SAMPLE BEFORE OILUTION WITH DILUENT

$>$ MEANS GREATER THAN

S MEANS LESS THAN THE SENSITIVITYOF THE SPECTROGRAPHIC PROCEDURE USED

ESULTS ARE CORRECT WITHIN A FACTOR OF COX, (ONE STANDARD DEVIATION) 


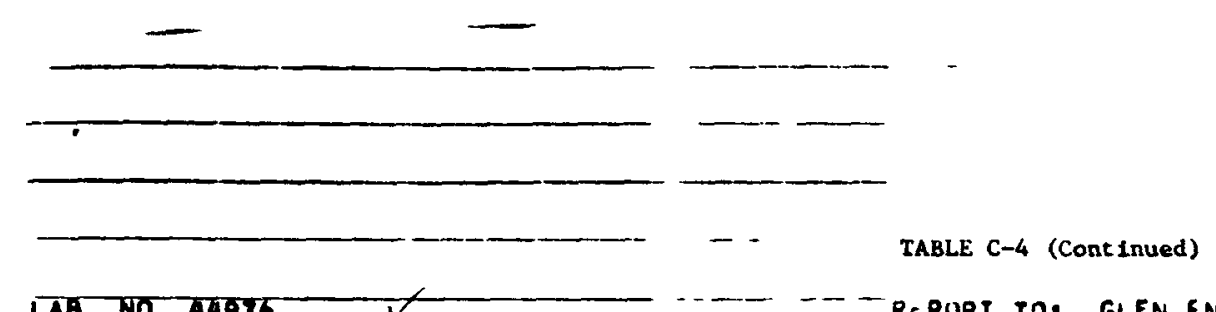

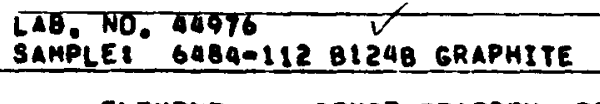
D.- - REPURT TOZ GLEN ENGLE PROJECT NO. 3224346005 GLEN DILUTION! 1.0000

DATEB $2-17-7 T$ ELEMENT CONEEMTRATION, PPH ELEMENT _ CONCENTRATION, PPM ELEMENT.

DATE NO. T6-
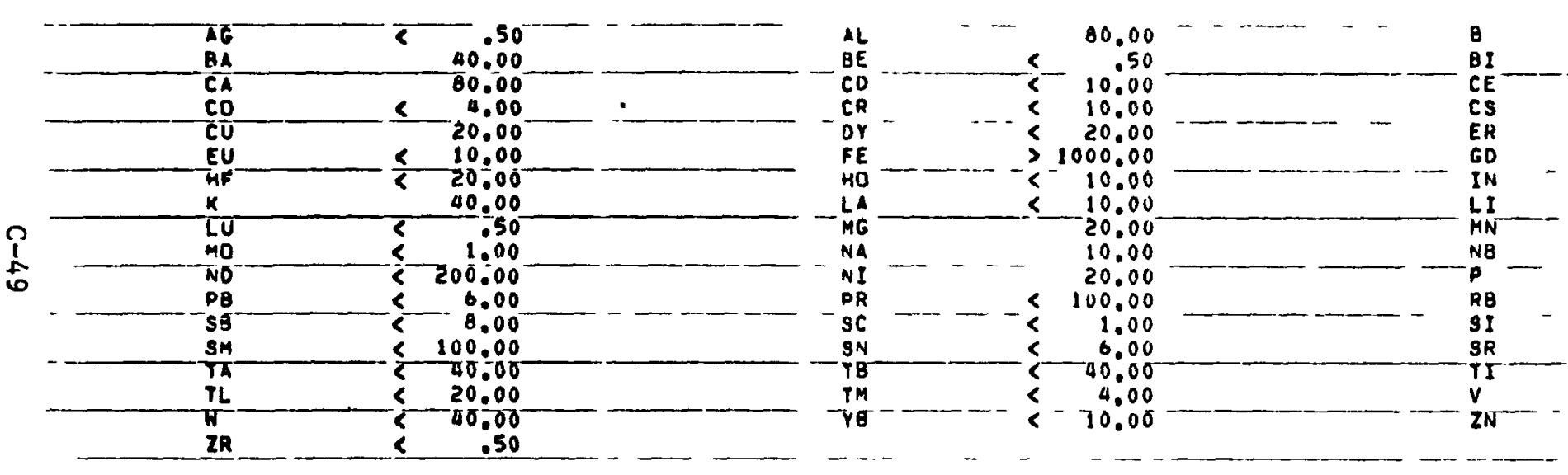
CONCENTRATION, PPM

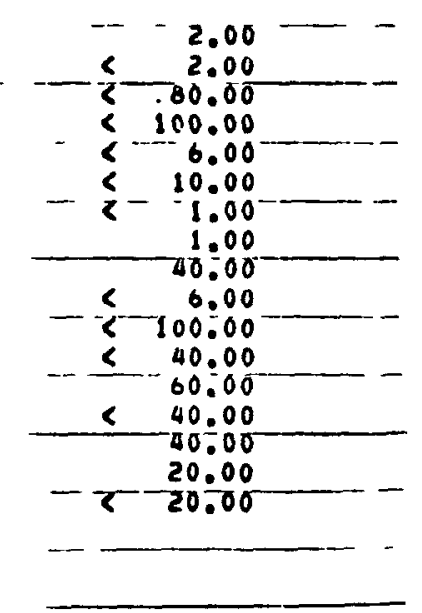

CONCENTRTTION BASEO ON OREINAL STAPLE BEFORE DILUTION WITH OILUENT

> MEANS GREATER THAN

WEANS LESS THAN THE SENSTTIVITYOF THE SPECTROGRAPHIC PROCEOURE USED

RESULTS ARE CORRECT WITHIN A FACTOR OF $40 X$. (ONE STANDARD DEVIATION) 


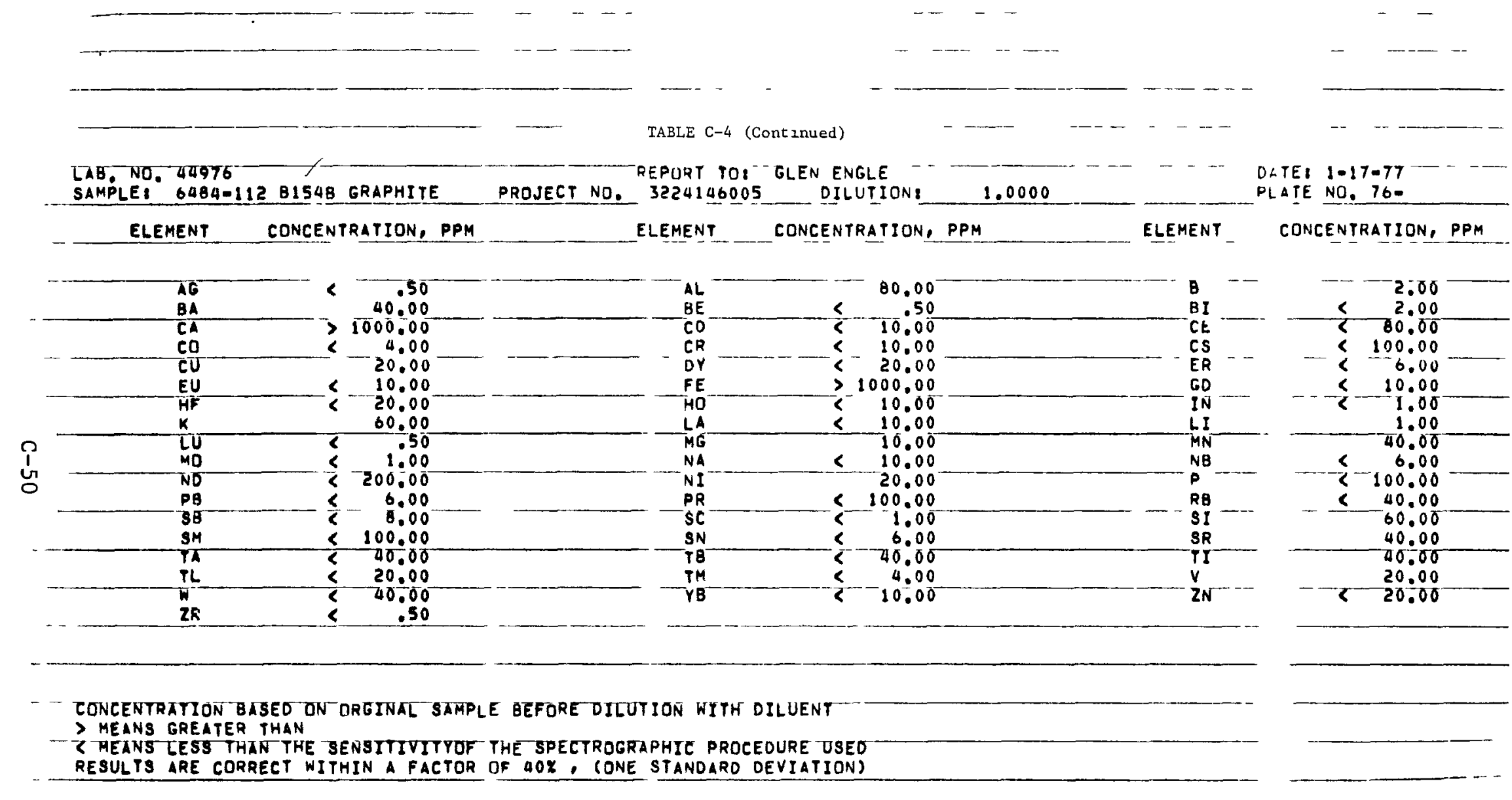




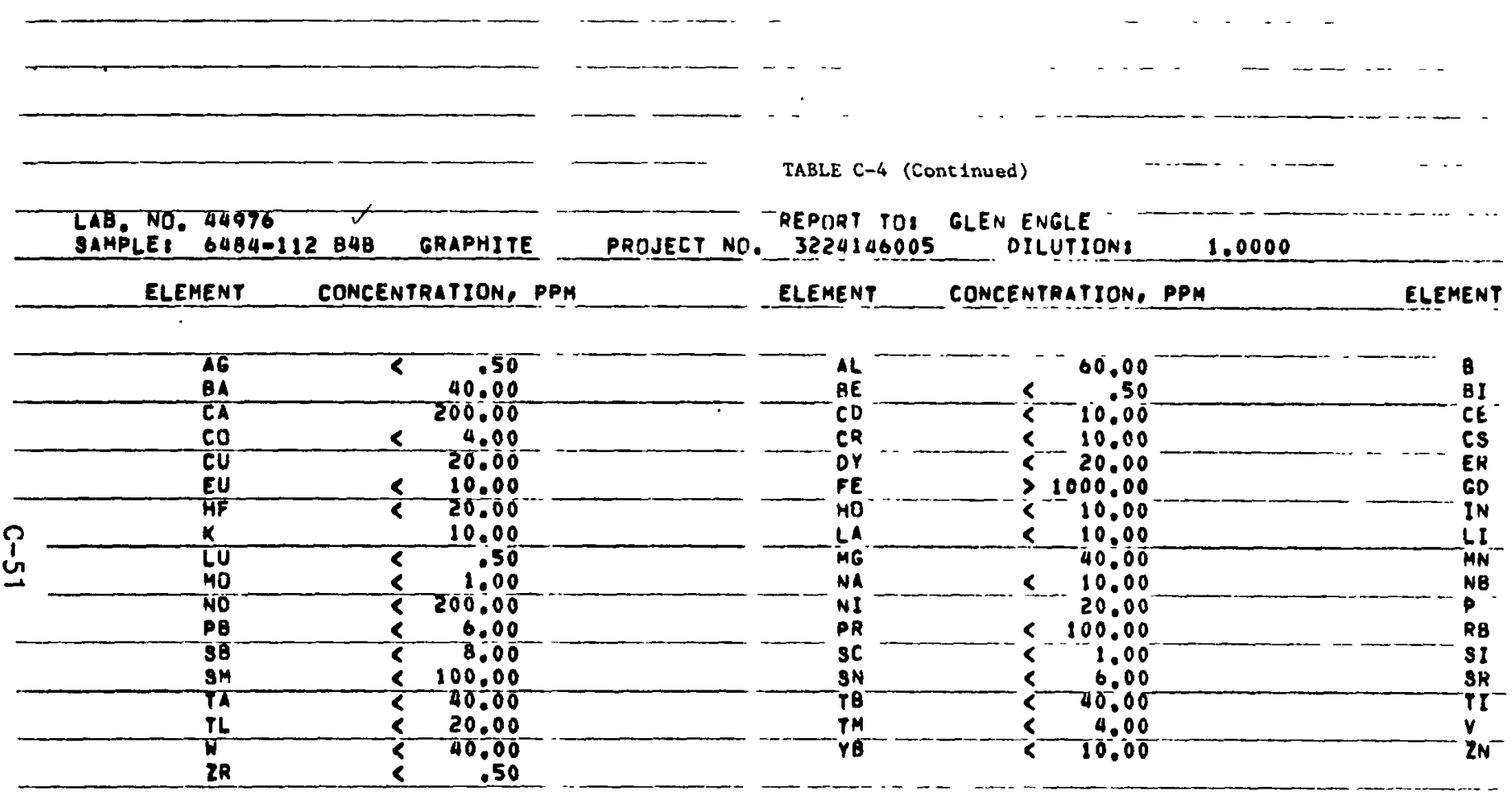

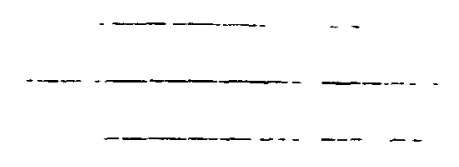

DATE: $1-\Gamma 7-77$

PLAIE NU. 76-

\section{CONCENTRATION, PPM}

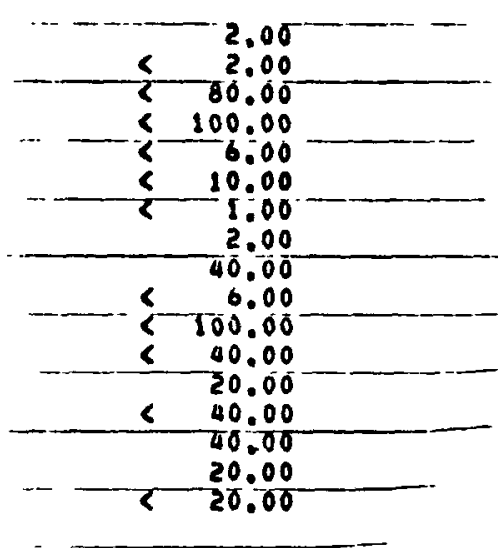

CONCENTRTTION BATSED ON ORGINAL SAMPLE BEFORE DILUTION WITH DILUENT

> MEANS GREATER THAN

CMEANS LESS THAN THE SENSITIVITYOF THE SPECTROGRAPHIC PROCEDURE USED

RESULTS ARE CORRECT WITHIN A FACTOR OF $40 \mathrm{O}$. (DNE STANOARD DEVIATIDN) 

TABLE C-4 (Cont 2nued)

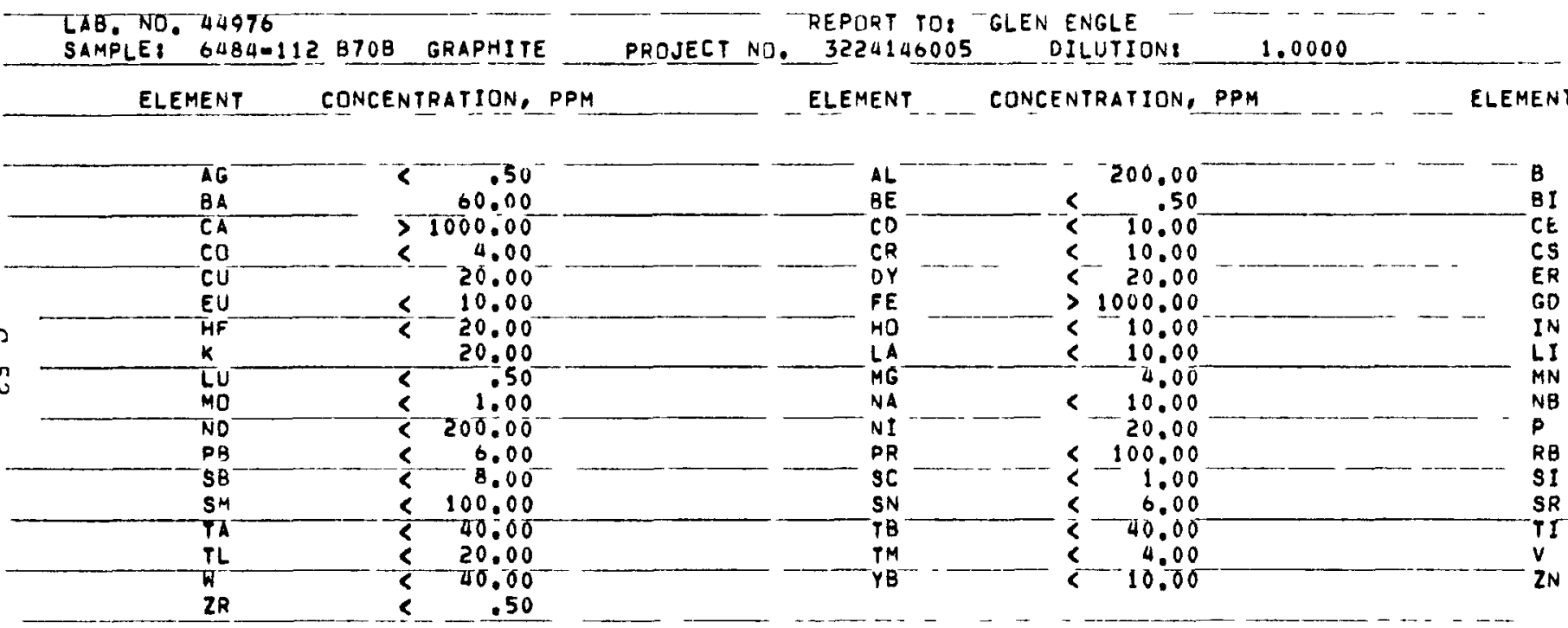

CONEENTRATION BASED ON ORGINAL

> MEANS GREATER THAN

RESUITS

RESULTS ARE CORRECT WITHIN A FACTOR DF $40 \%$, (ONE STANDARD DEVIATION)
DATE: $1-17-77 \ldots$

PLATE NO. 76-

CONCENTRATION, PPM

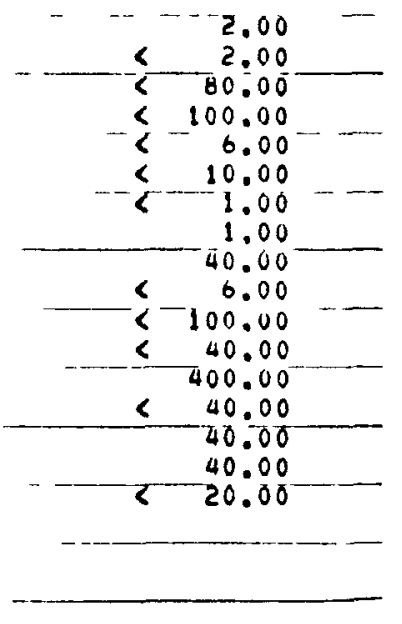

- 
LAB NO: 44976 MAPLE: 6484-112 MI008 GRAPHITE

AMPLE \& 6484-112 M1008 GRAPHITE ELEMENT__CONCENTRATION, PPM

PROJECT NO. 3224146005 GLEN ENGLE

ELEMENT CONCENTRATION, PPM

1.0000

ELEMENT

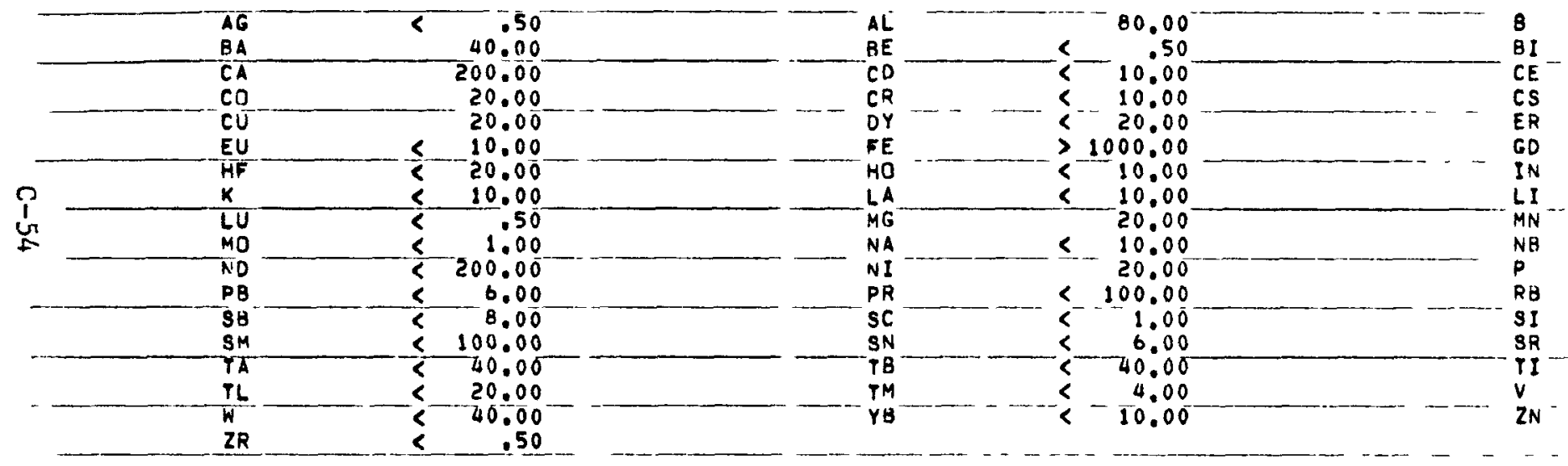

CONCENTRATION BASED DN OORGINAL SAMPLE BEFORE OILUTIONTWITH DILUENT

ᄀ MEANS GREATER THAN

C MEANNS LESS THAN THE SEÑSITIVI TYOF THE SPECTROGRAPHIC PROCEDURE USEO

RESULTS ARE CORRECT WITHIN A FACTOR OF $40 \%$, (ONE STANDARD DEVIATION)
TTE: $1-17-77$

CONCENTRATION, PPM

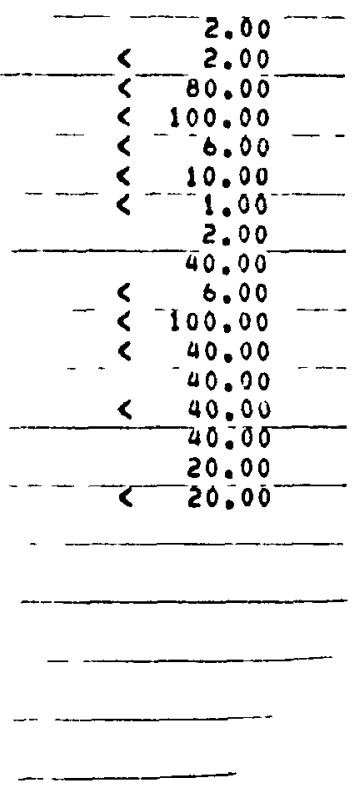




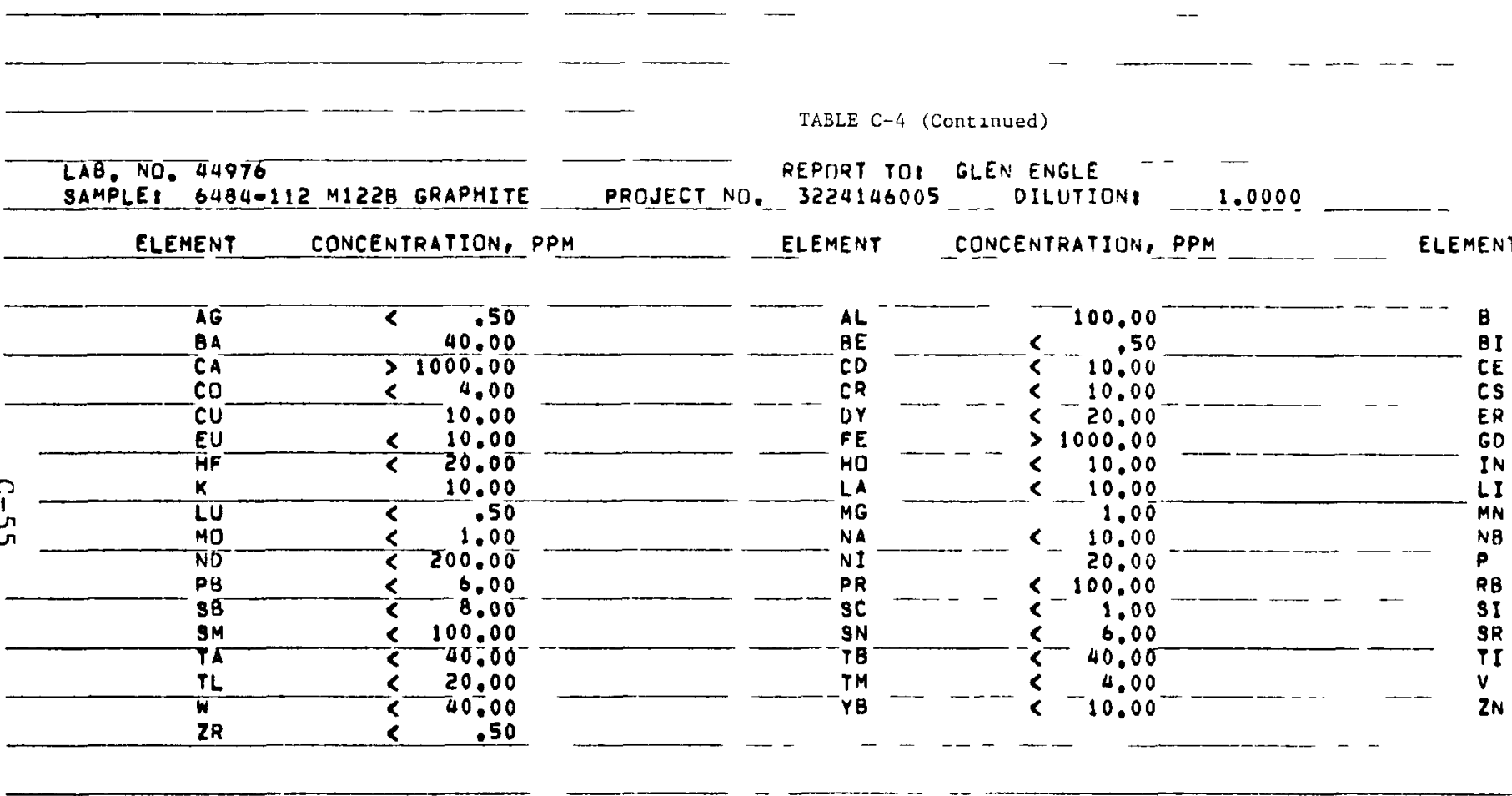

CONCENTRATION BASED ON ORGINAL SAMPLE BEFORE DILUTION WITH DILUENT

$>$ MEANS GREATER THAN

R MEANS LESS THAN YHE SENSITIVITYOF THE SPECTRUGRAPHIC PROCEDURE USED

RESULTS ARE CORRECT WITHIN A FACTOR OF $40 X$. (ONE STANDARD DEVIATION) 


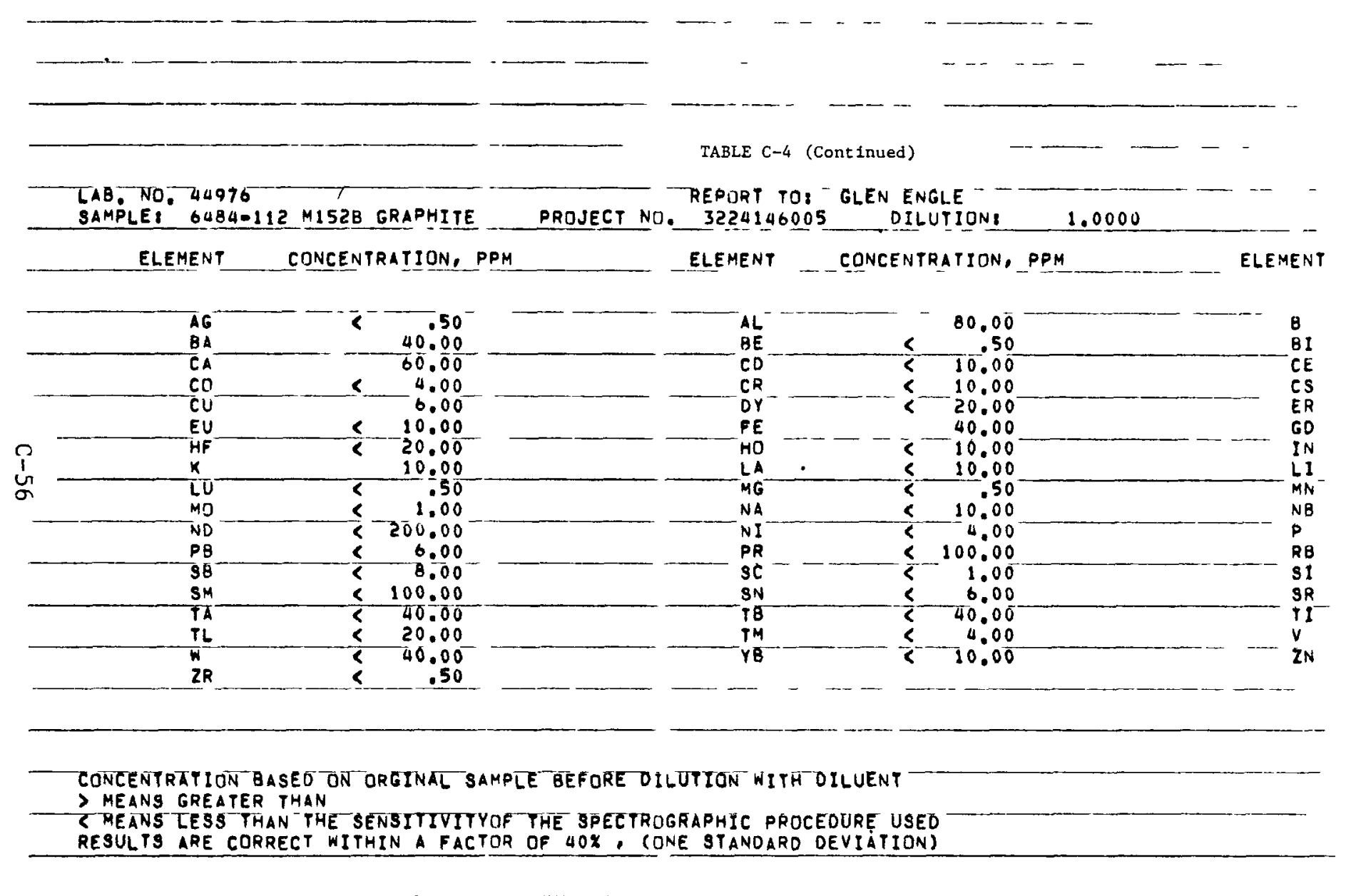

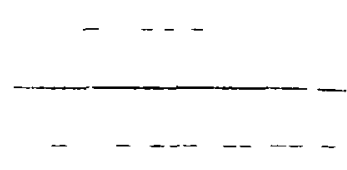

DATE: $1-17-77^{-}-\cdots$ PLATE NO, 76-

CONCENTRATION, PPM
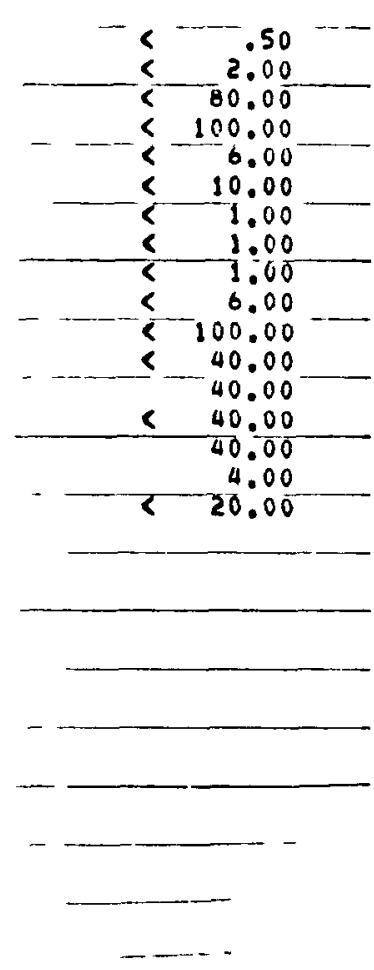

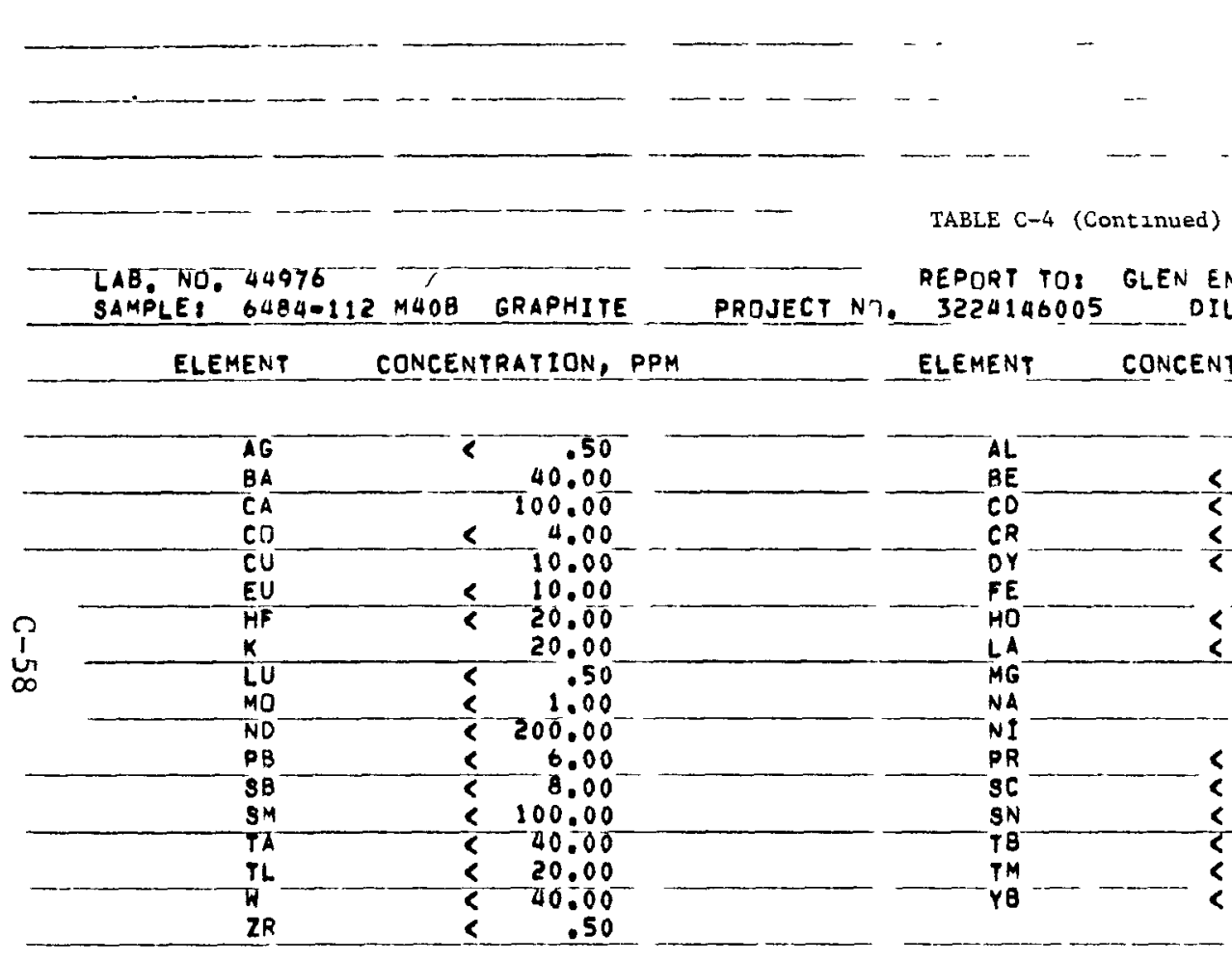

DATE, $1-17-77$

PLATE NO. $76-$

CONCENTRATION, PPM

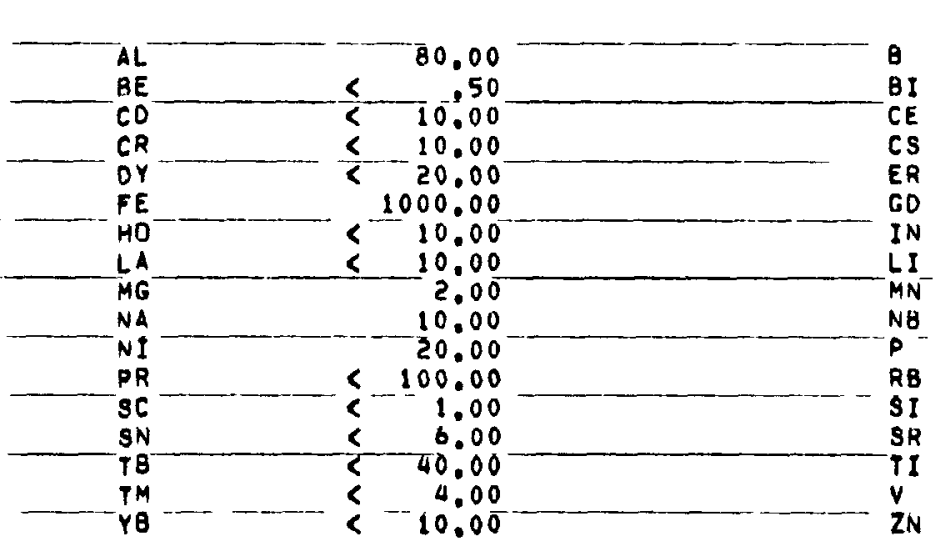

CONCENTRATION BASEO OÑ ORGINAL SAMPLE BEFORE DILOTION WITH DILUENT

> MEANS GREATER THAN

C MEANS LESS THAN THE SENSITIVITYOF THE SPECTROGRAPHIC PROCEOURE USEO

RESULTS ARE CORRECT WITHIN A FACTOR OF $40 \mathrm{X}$, (ONE STANDARD DEVIATION)

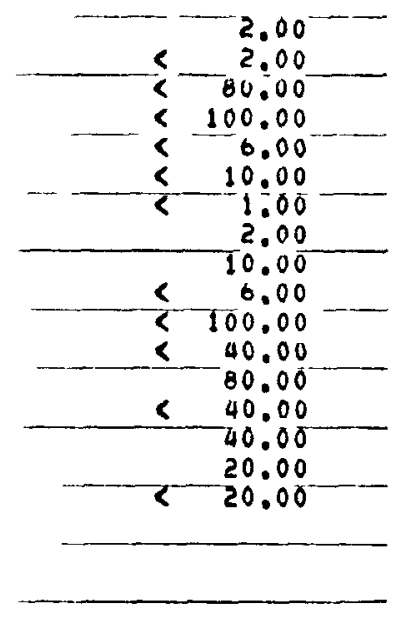




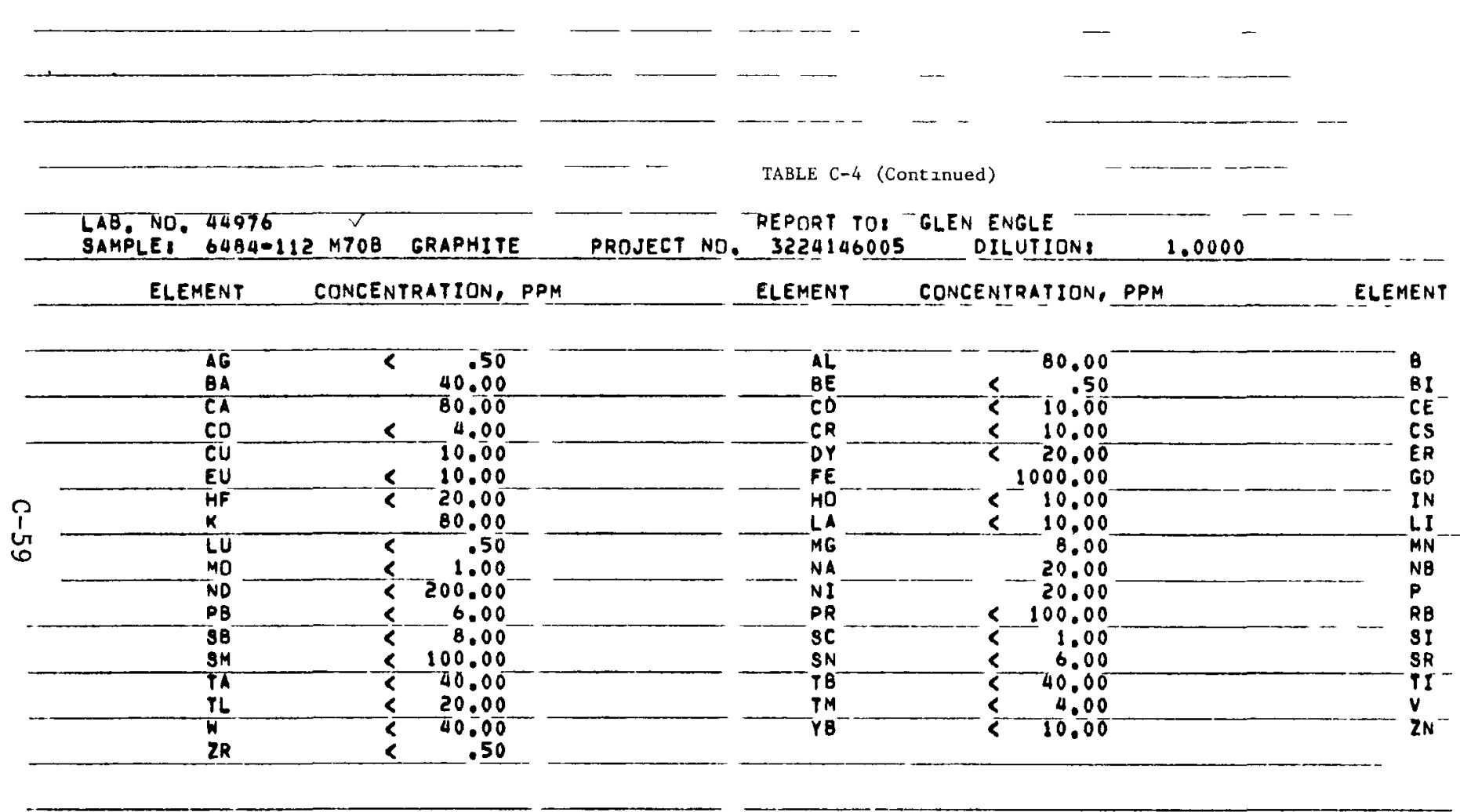

DATE, $1-17-77$ PLATE NO. 70-

CONCENTRATION, PPM

CONCENTRATION BASED ON ORGINAL SAMPLE GEFORE DILUTYON WITH DILUENT

> MEANS GREATER THAN

MEANS LESS TAAN TAE SENSITIVITPOF THE SPECTROGRAPHIC PROCEDURE USEO

RESULTS ARE CORRECT WITHIN A FACTOR OF WOX, (ONE STANOARD DEVIATION)
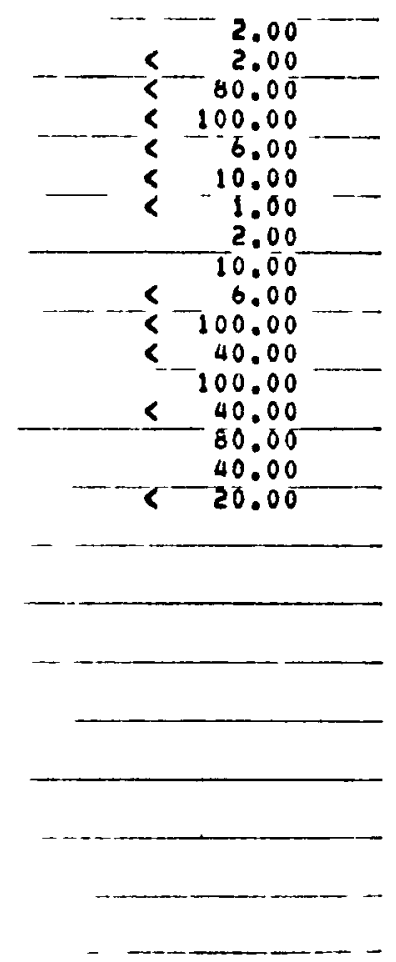


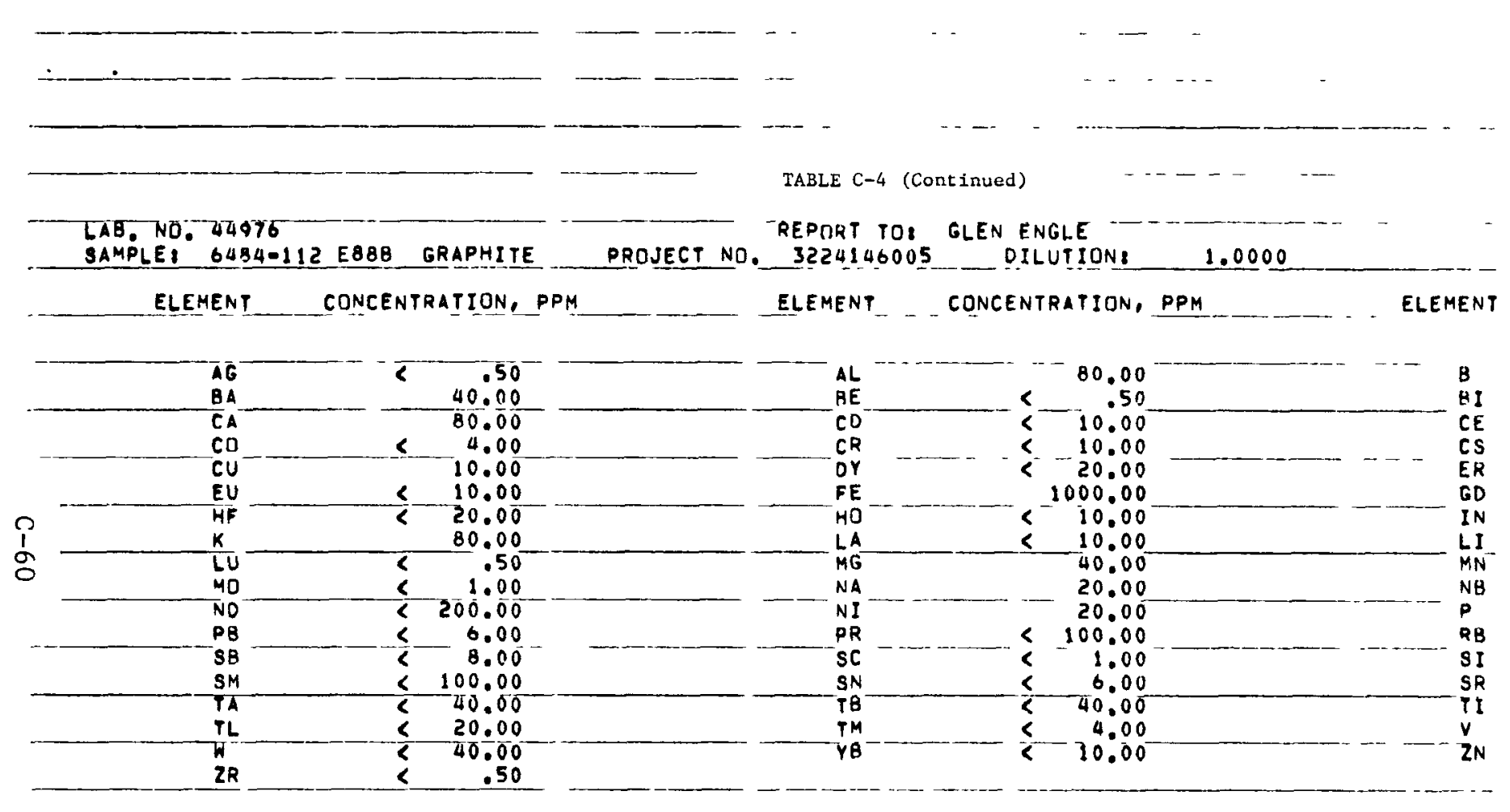

DATE: $1-17-77$

PLATE NO. 76-

CONCENTRATION, PPM

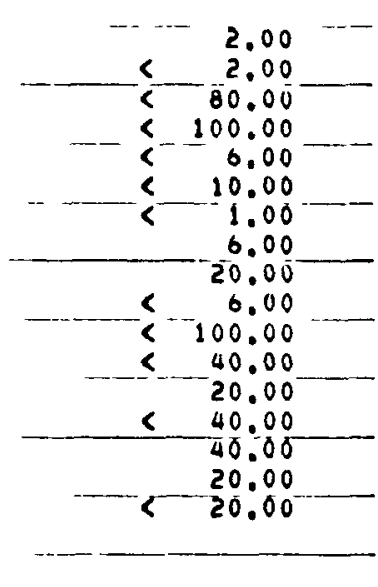

CONCENTRATION BASED ON ORGINAL SAMPLE BEFORE DILUTION WITH DILUENT

> MEANS GREATER THAN

C MEANS GREATER THAN ON ORGINAL SAMPLE BEFORE DILUTION WITH DILUENT

RESULTS ARE CORRECT WITHIN A FACTOR OF $40 \mathrm{X}$, (OVE STANDARO OEVIATION) 


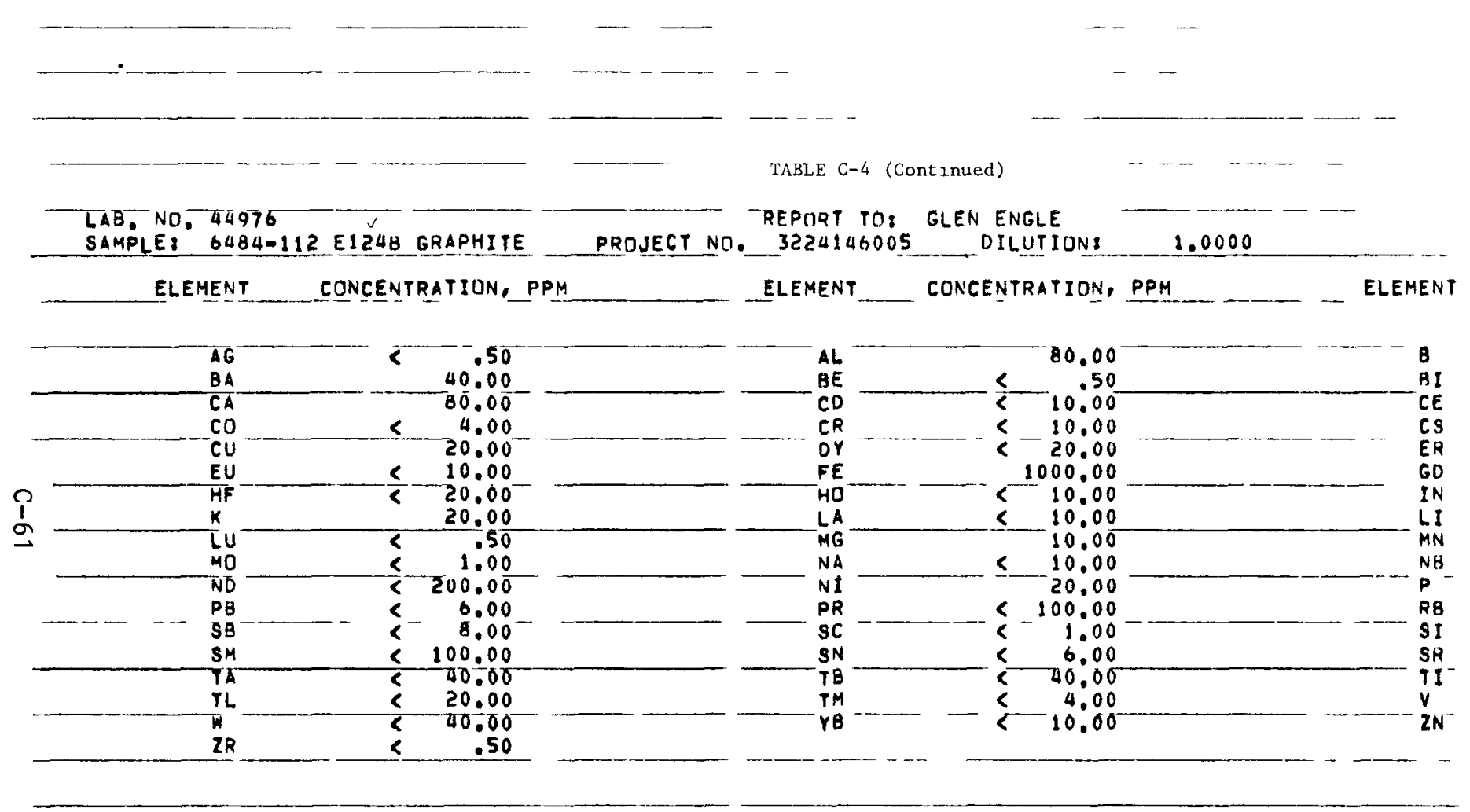

CONCENTRATION BASEO ON ORGINAL SAMPLE BEFORE DILUTION WITH DILUENT

\ MEANS GREATER THAN

MEANS LESS THAN THE SENSITIVITYOF THE SPECTROGRAPHIC PROCEDURE USEO

RESULTS ARE CORRECT WITHIN A FACTOR OF $40 \mathrm{X}$. (UNE STANDARD DEVIATION) 


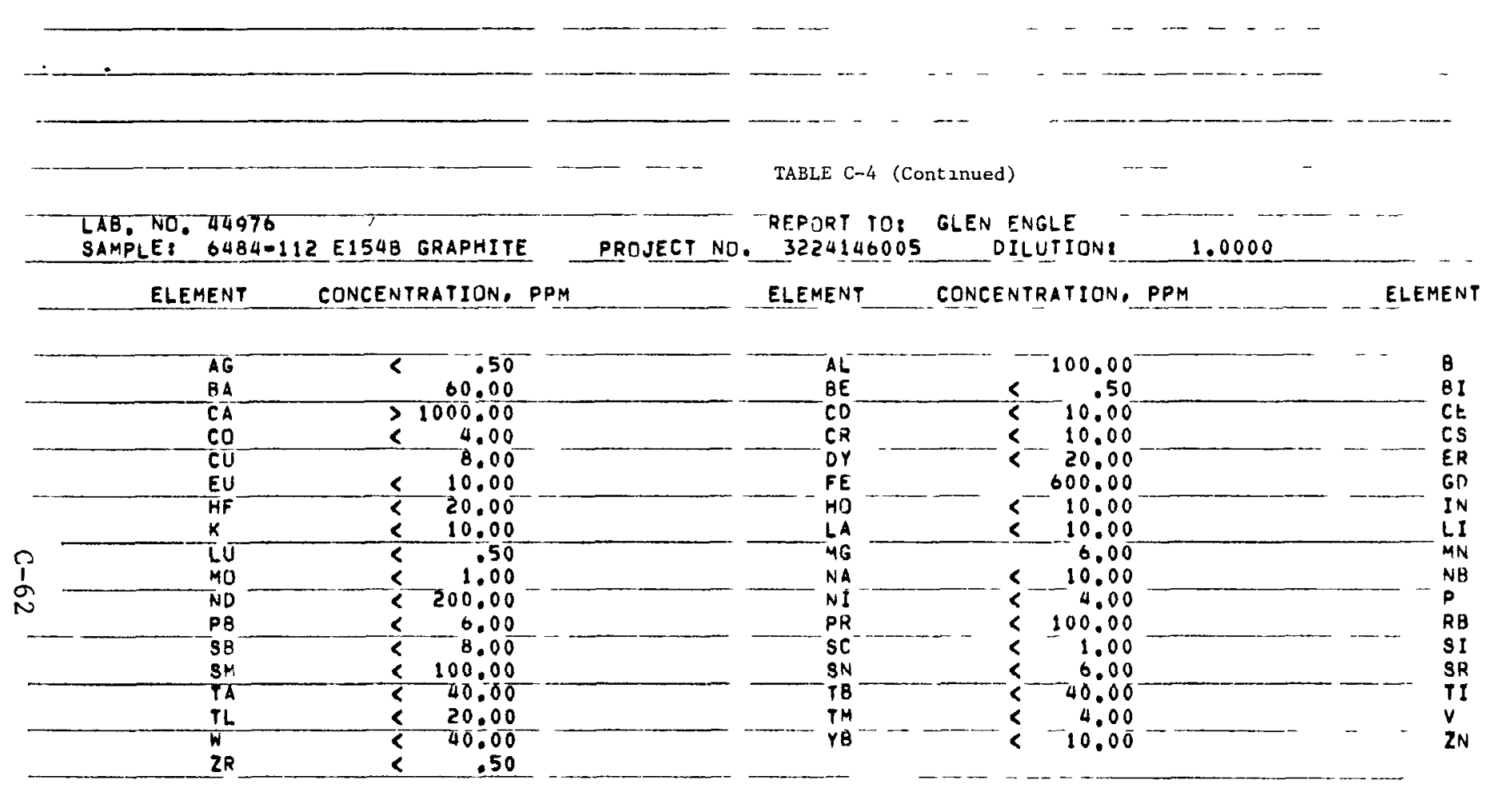

DATE: $1-17-77$

PLATE NO. 76-

CONCENTRATION, PPM

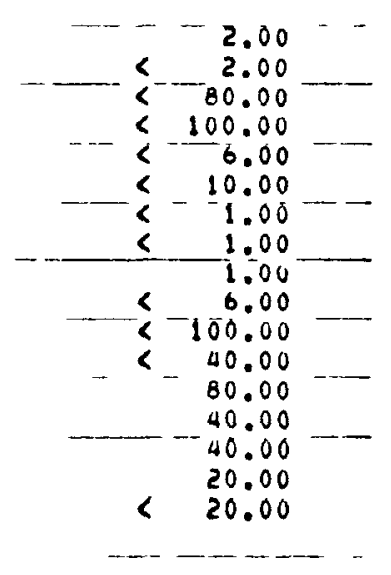

CONCENTRATI ON BASEO ON ORGINAL SAMPLE BEFORE DILUTION WITH DILUENT

> MEANS GREATER THAN

\MEANS [ESS THAN THE SENSITIVITYOF THE SPECTROGRAPHIE PROCEDORE USED

RESULTS ARE CORRECT WITHIN A FACTDR OF $40 X$, (ONE STANDARO DEVIATION)
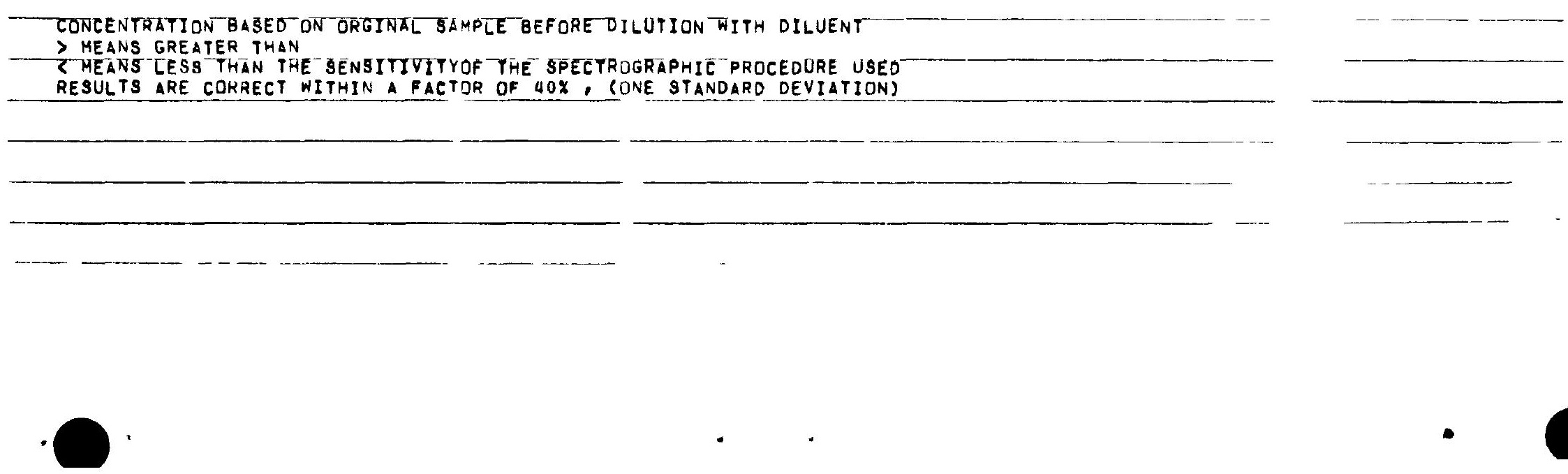


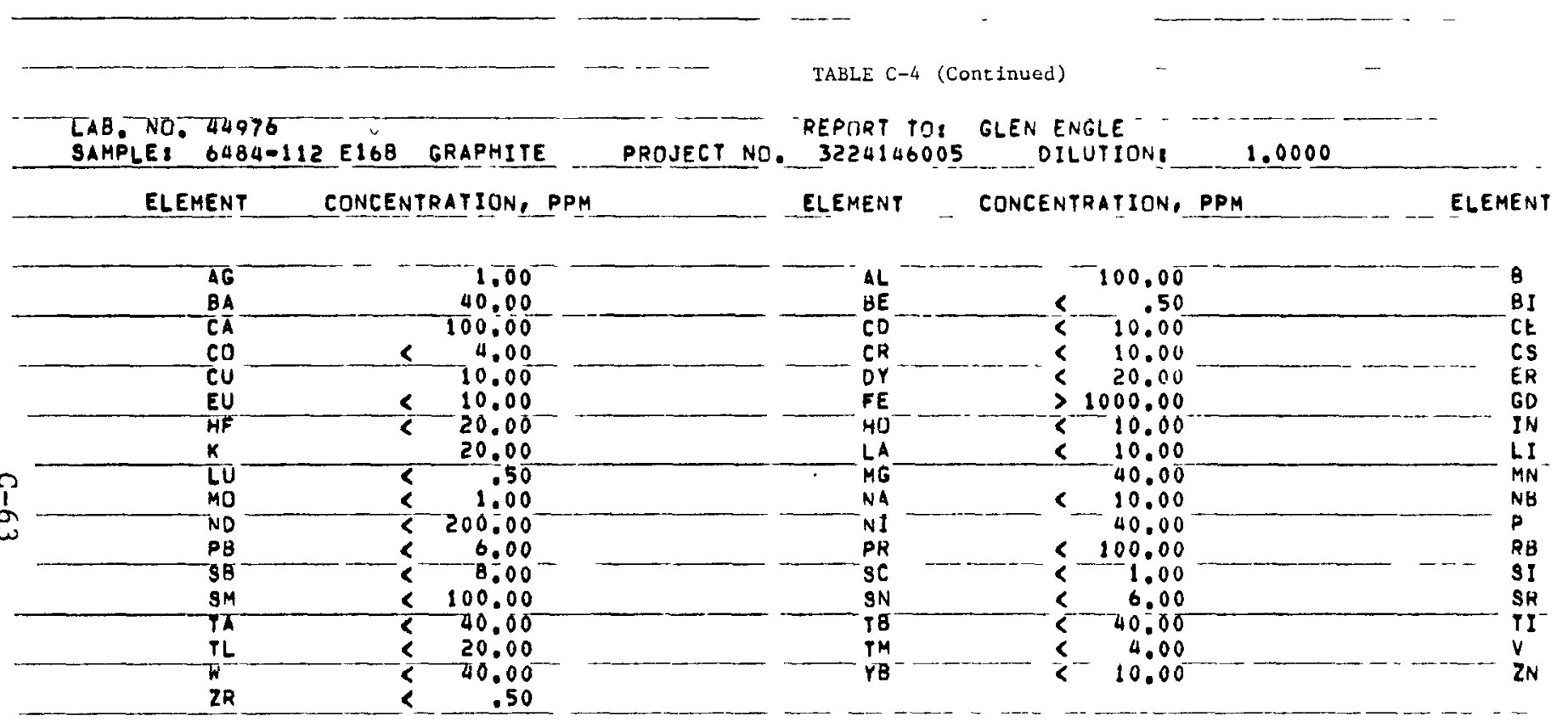

CONCENTRATION BASED ON ORGINAL SAMPLE BEFORE DILUTION WITH DILUENT

> MEANS GREATER THAN

RESUTS

RESULTS ARE CORRECT WITHIN A FACTOR OF $40 X$, (ONE STANDARD DEVIATIUN)

OATE, $1-17-77$
PLATE NO, TE-

CONCENTRATION, PPK
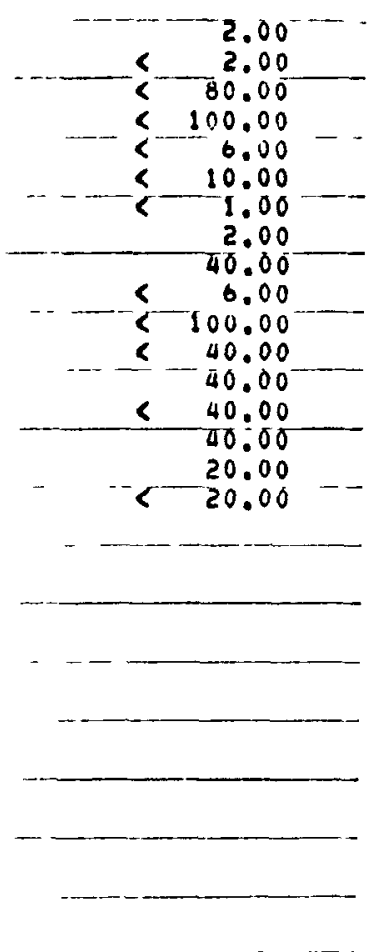


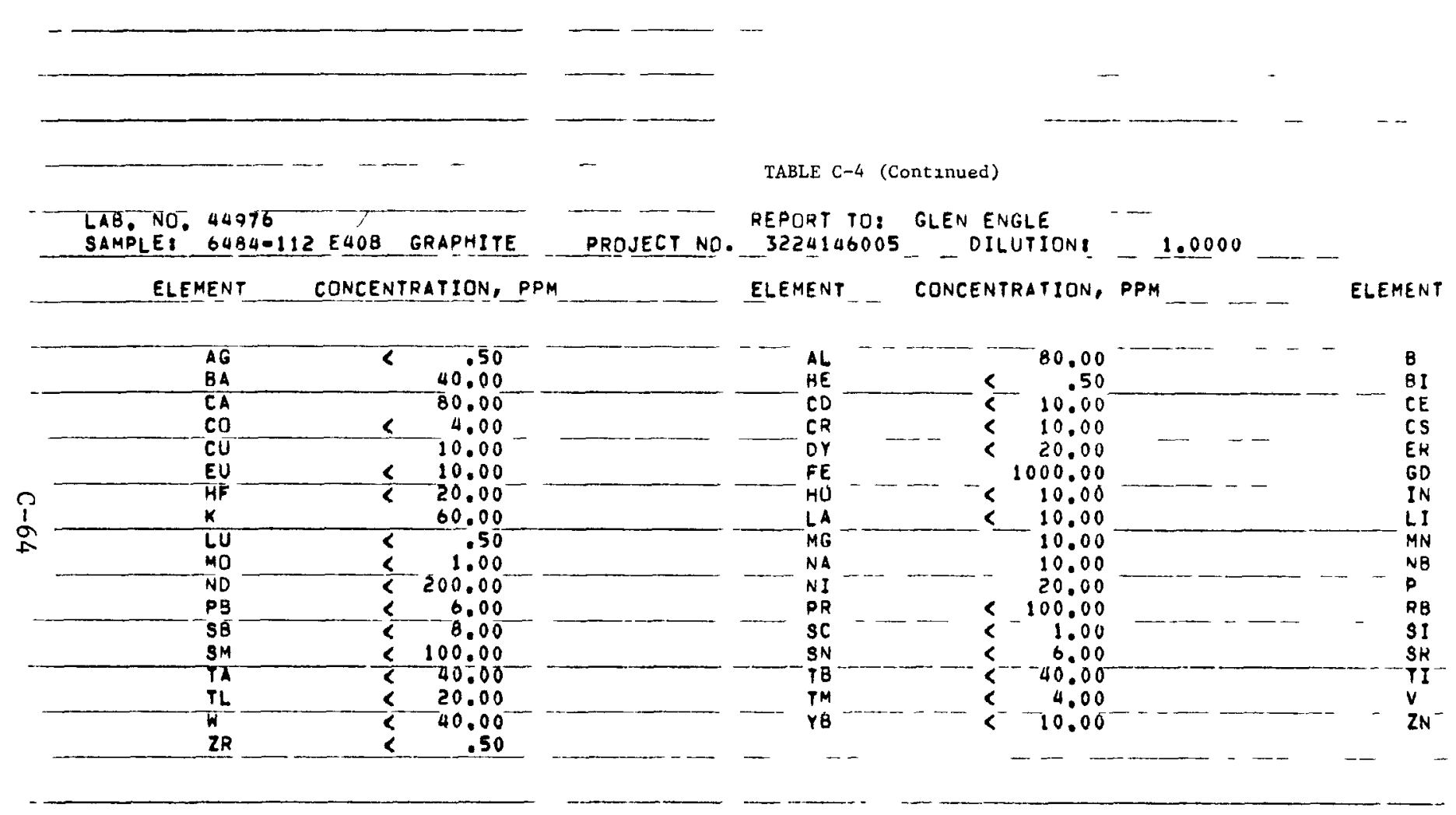

OATE: $1-17-77$

PLATE 10.760

CONCENTRATION, PPM

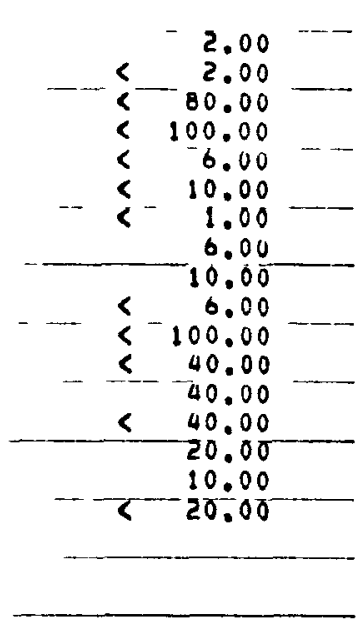

CONCENTRATION BASEO ON ORGINAL SAMPLE BEFORE OILUTION WITH DILUENT

$\checkmark$ MEANS GREATER THAN

TMEANNS LESS THAN THE SENGITIVITYUF THE SPECTROGRAPHIC PROCEOURE USËD

RESULTS ARE CORRECT WITHIN A FACTOR OF $40 \%$. (ONE STANOARD DEVIATION) 
APPENDIX D

GRADE HLM DATA

This appendix contains complete data sets for the bulk density, tensile properties, flexural strength, compressive properties, and chemical impurity content of grade HLM graphite. 
TABLE $D-1$

TENSILE PROPERTIES OF HLM GRAPHITE

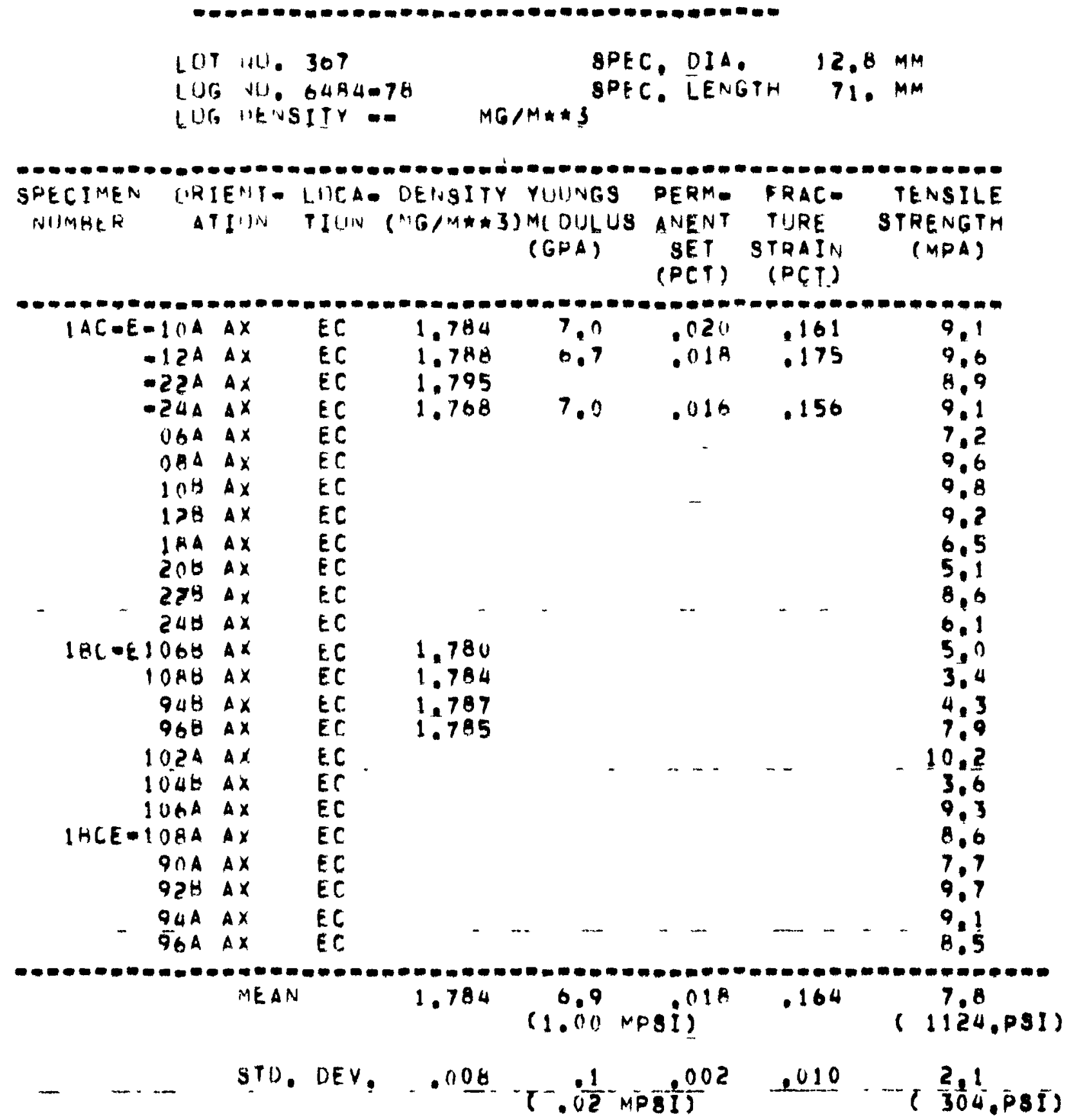


TABLE D-1 (Continued)

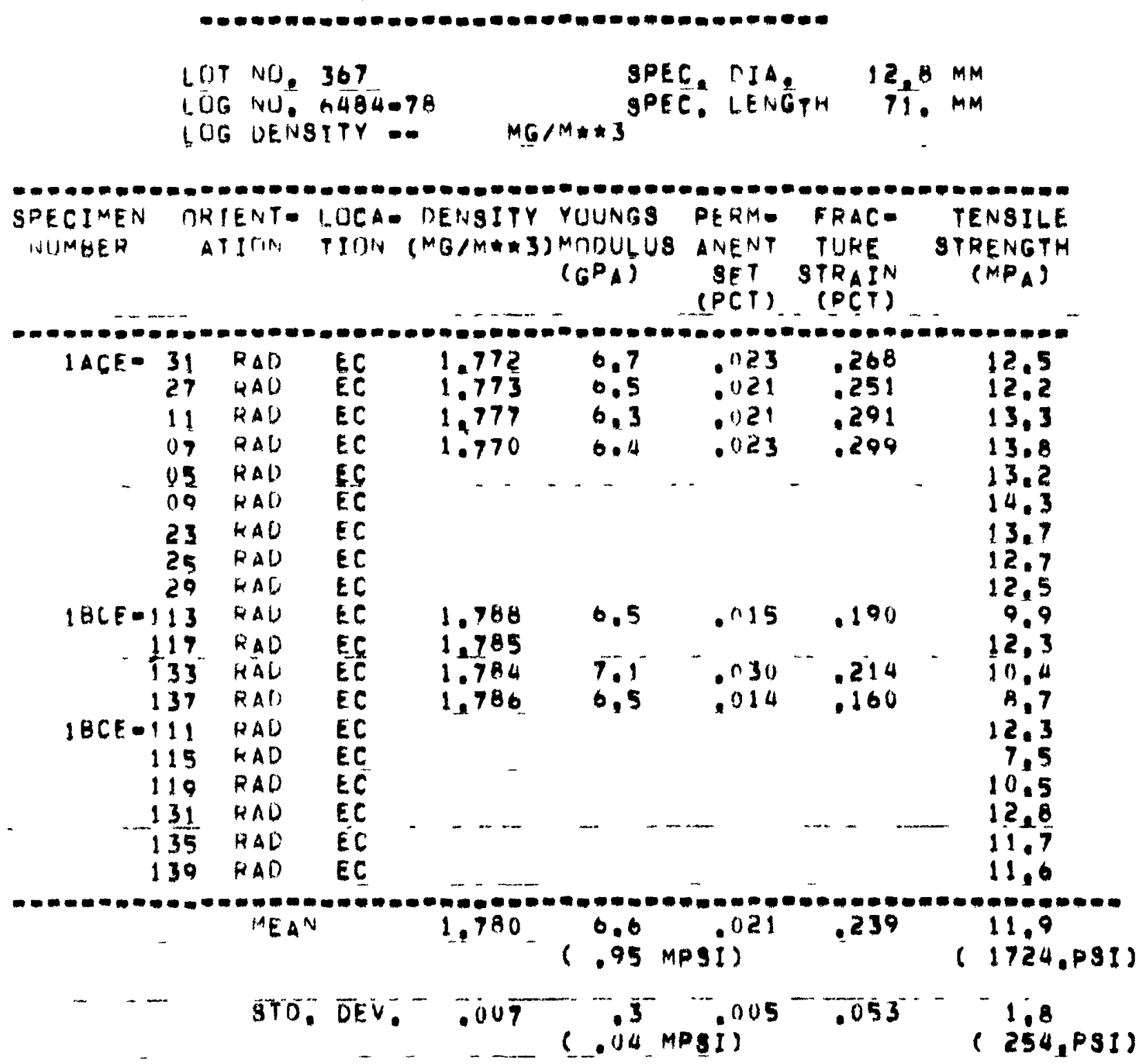


TABLE D-1 (Continued)

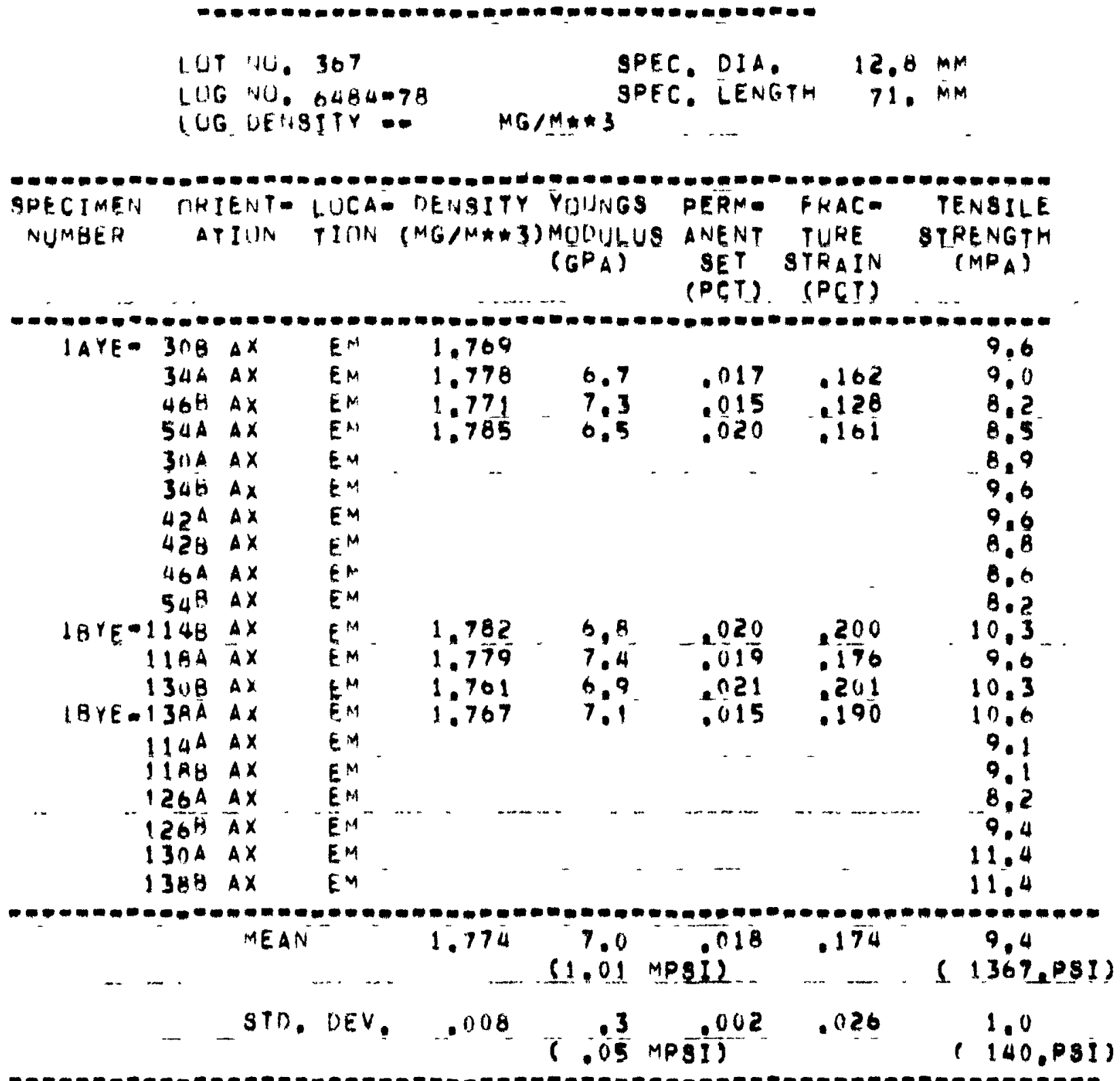


TABLE D-1 (Continued)

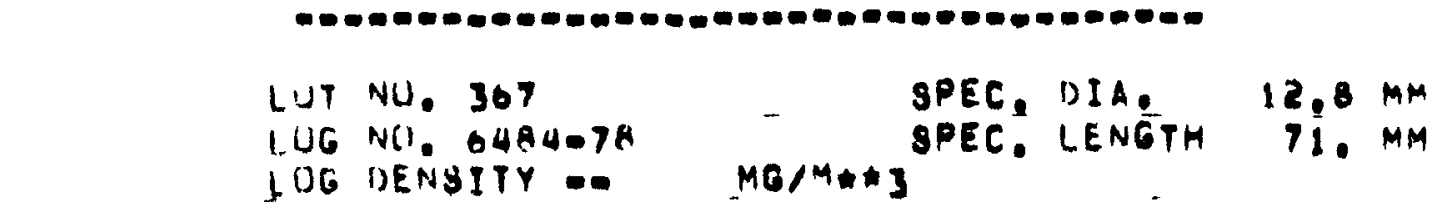

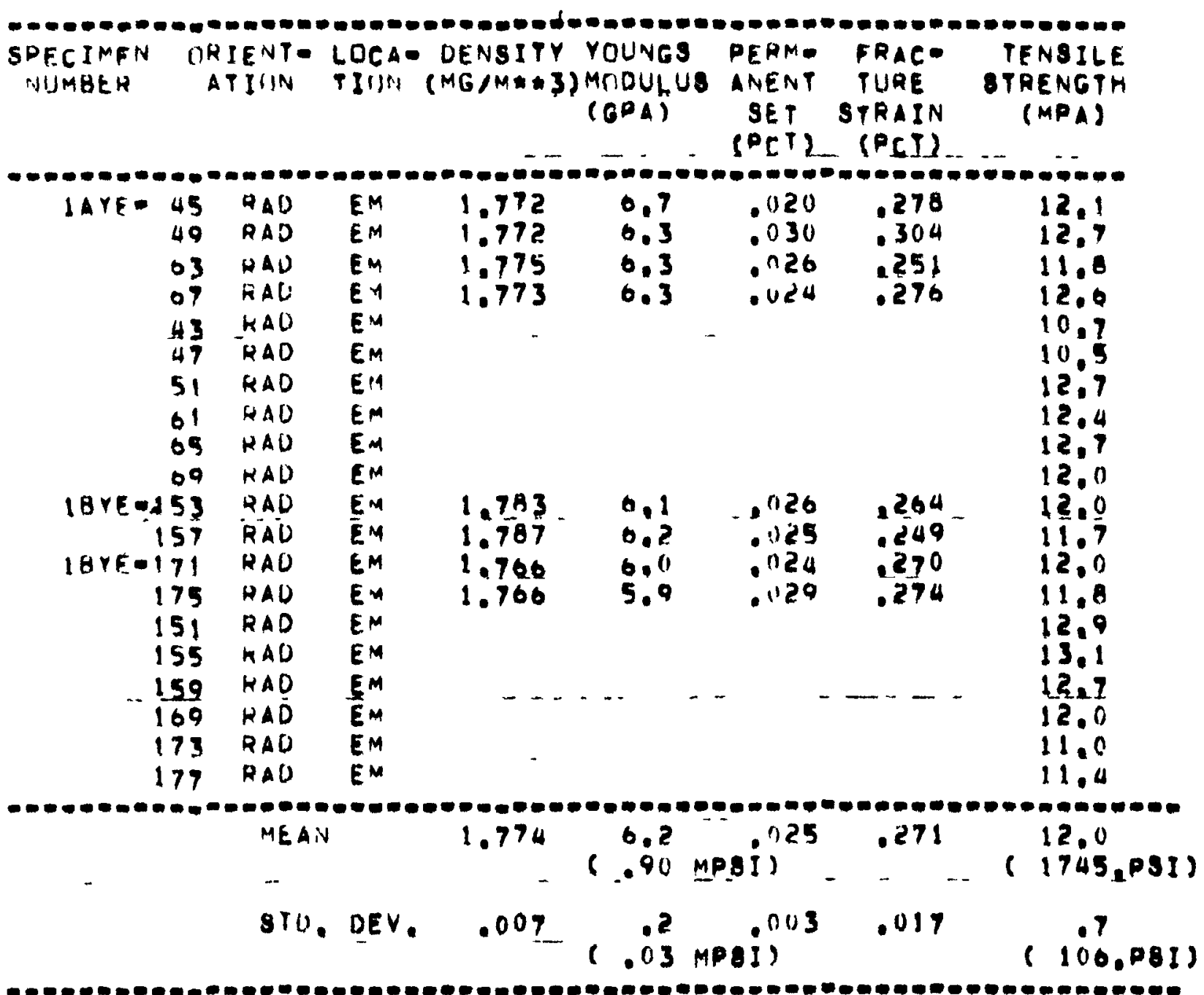


TABLE D-1 (Continued)

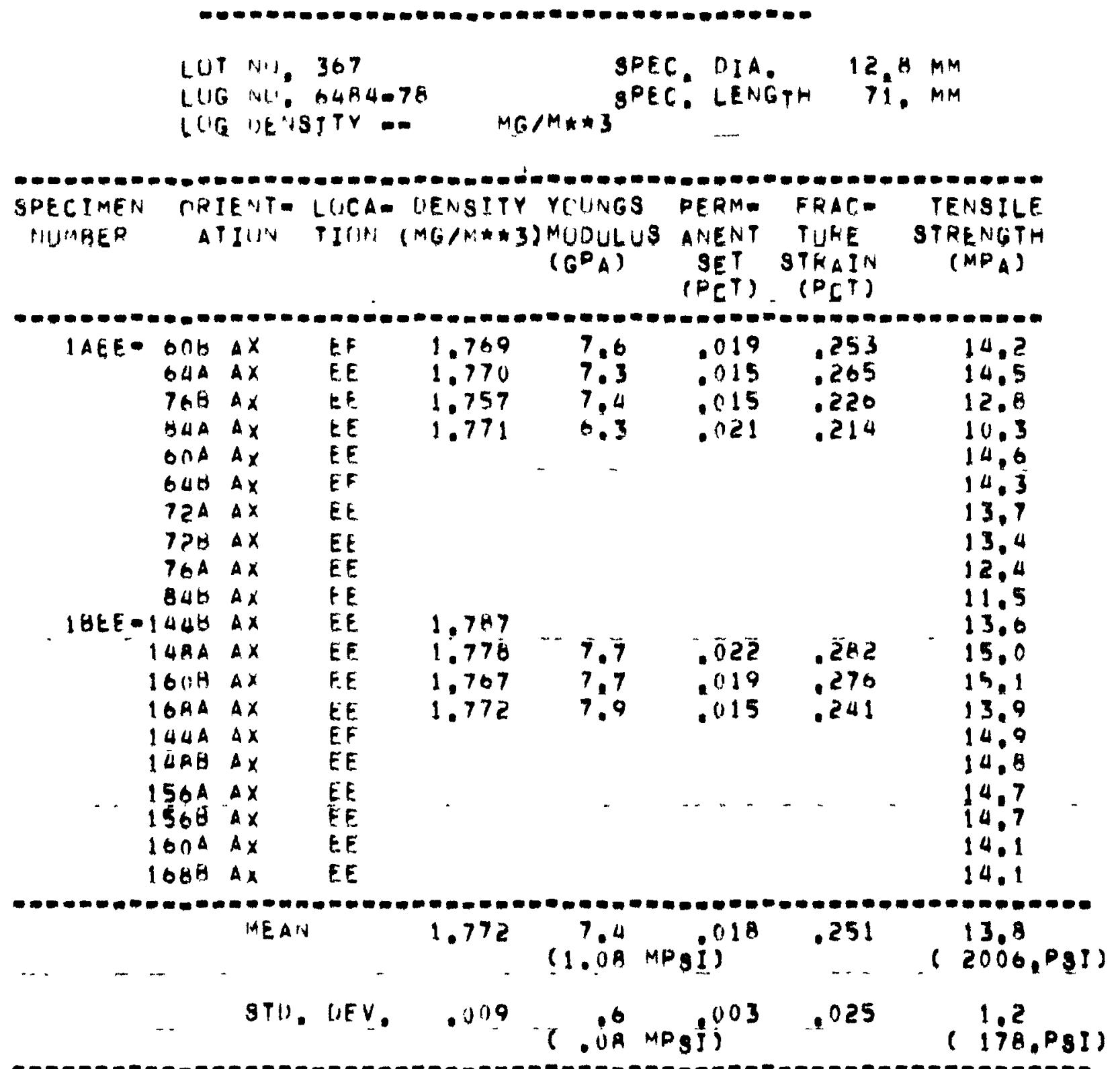


TABLE D-1 (Continued)

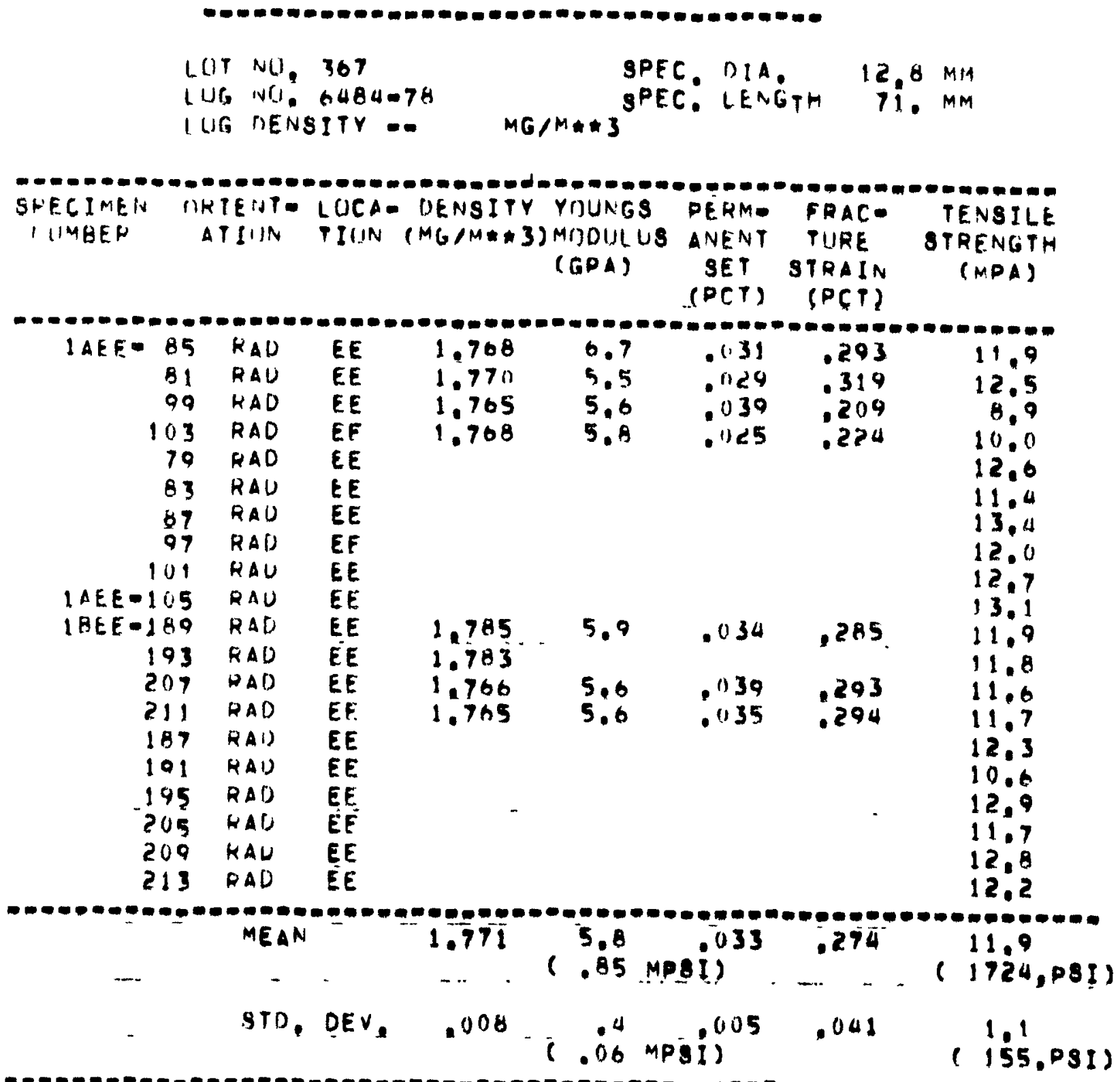


TABLE D-1 (Continued)

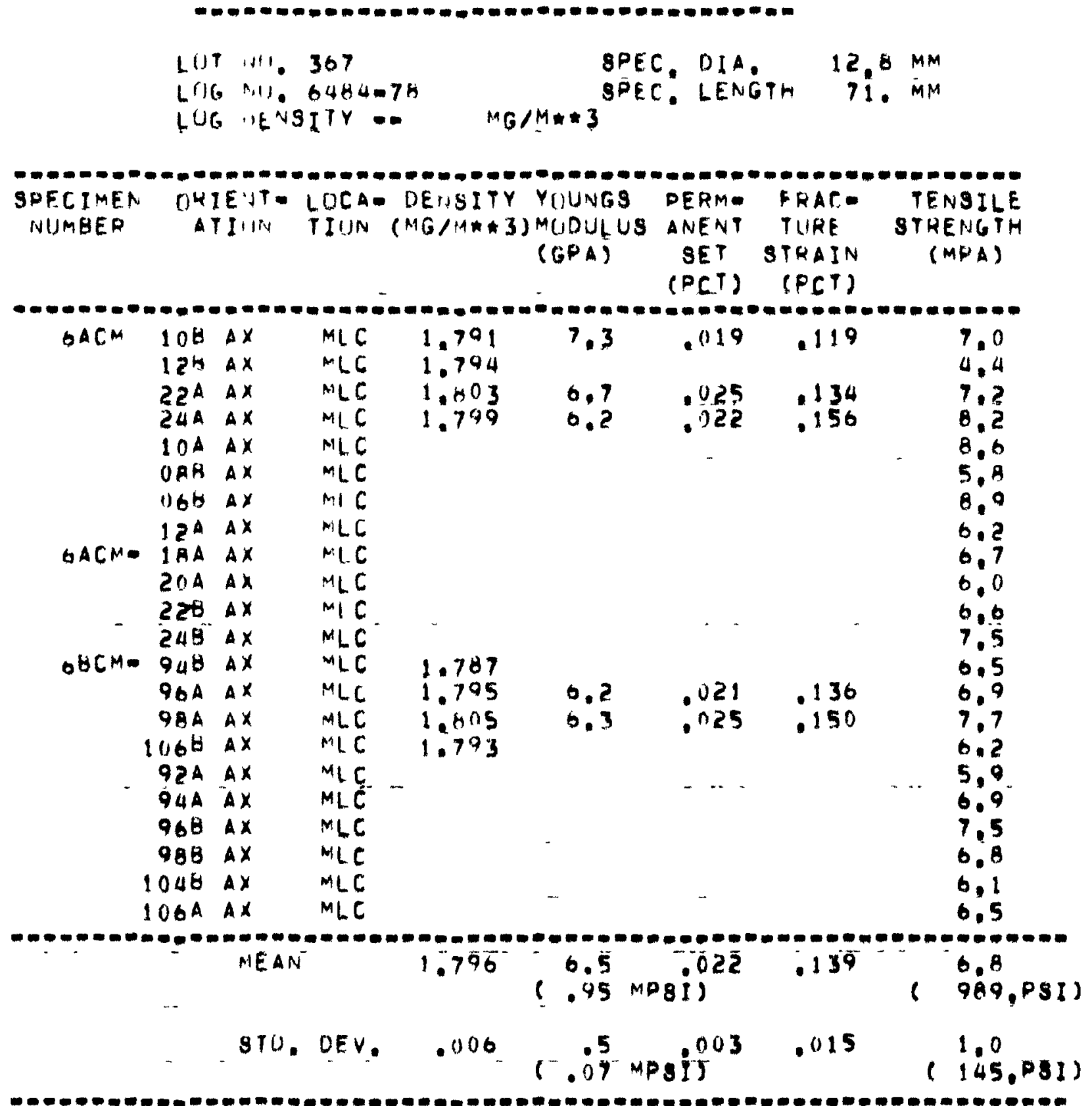


TABLE D-1 (Continued)

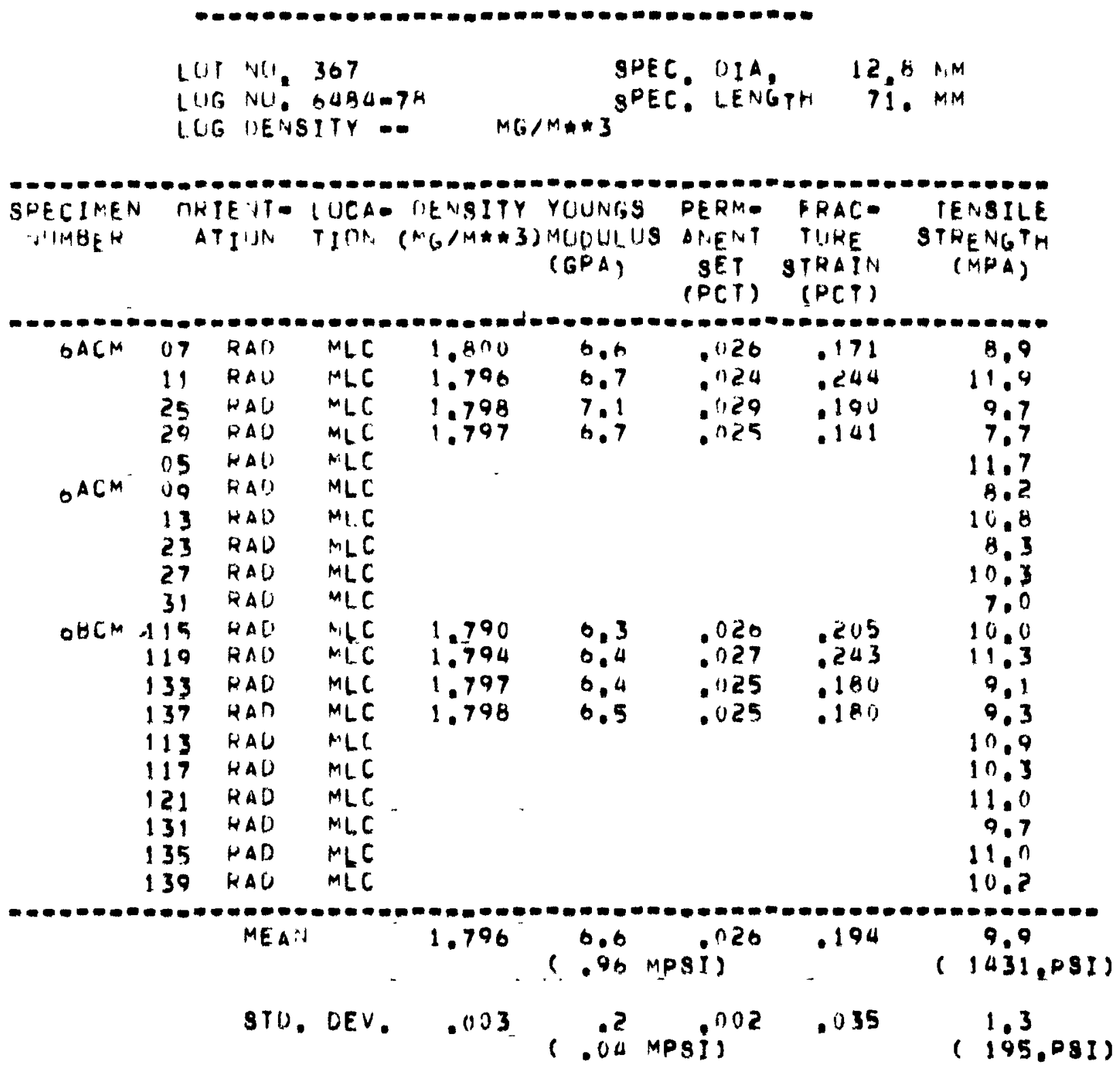


TABLE D-1 (Continued)

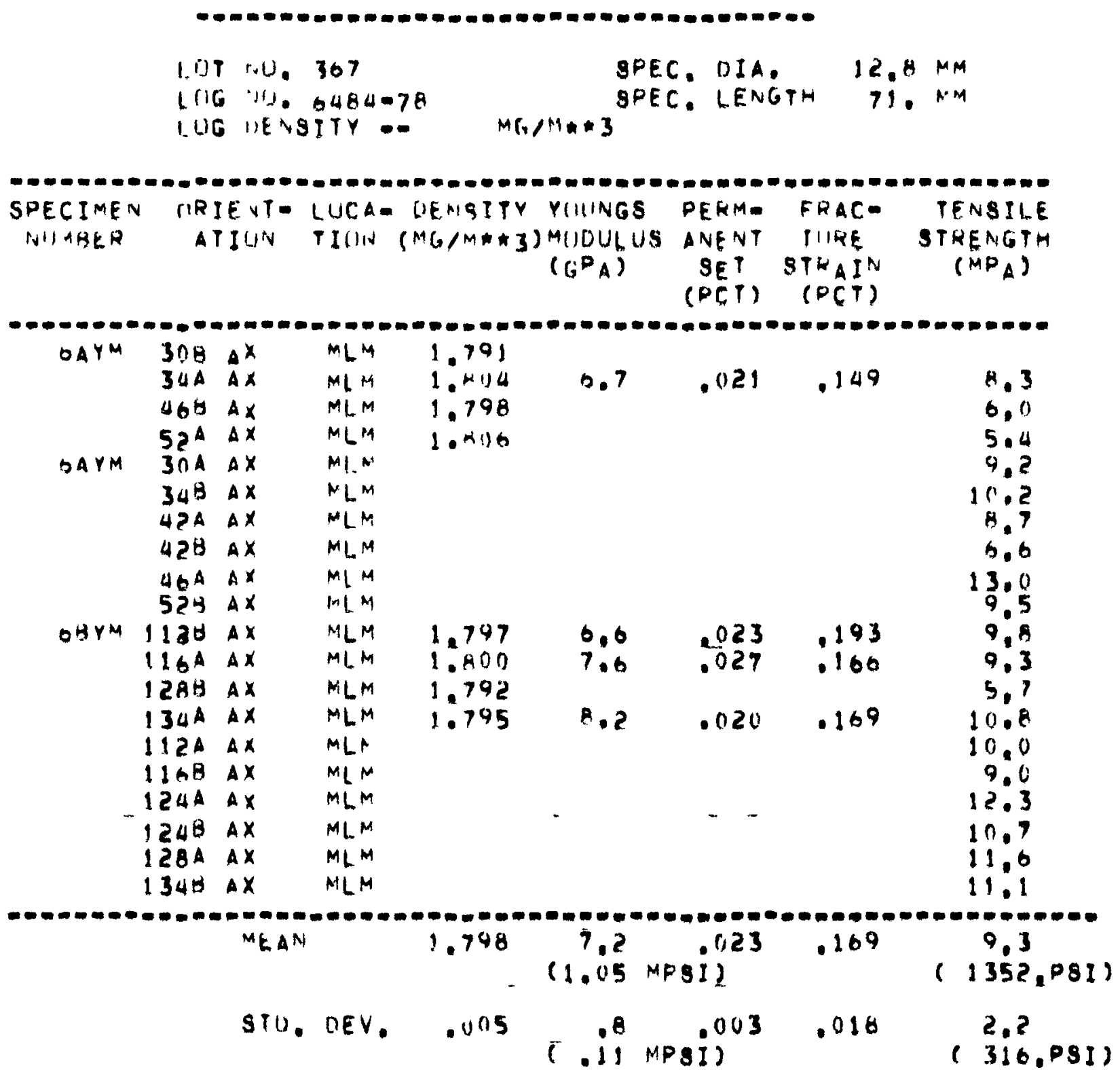

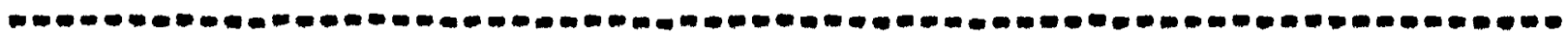


TABLE D-1 (Continued)

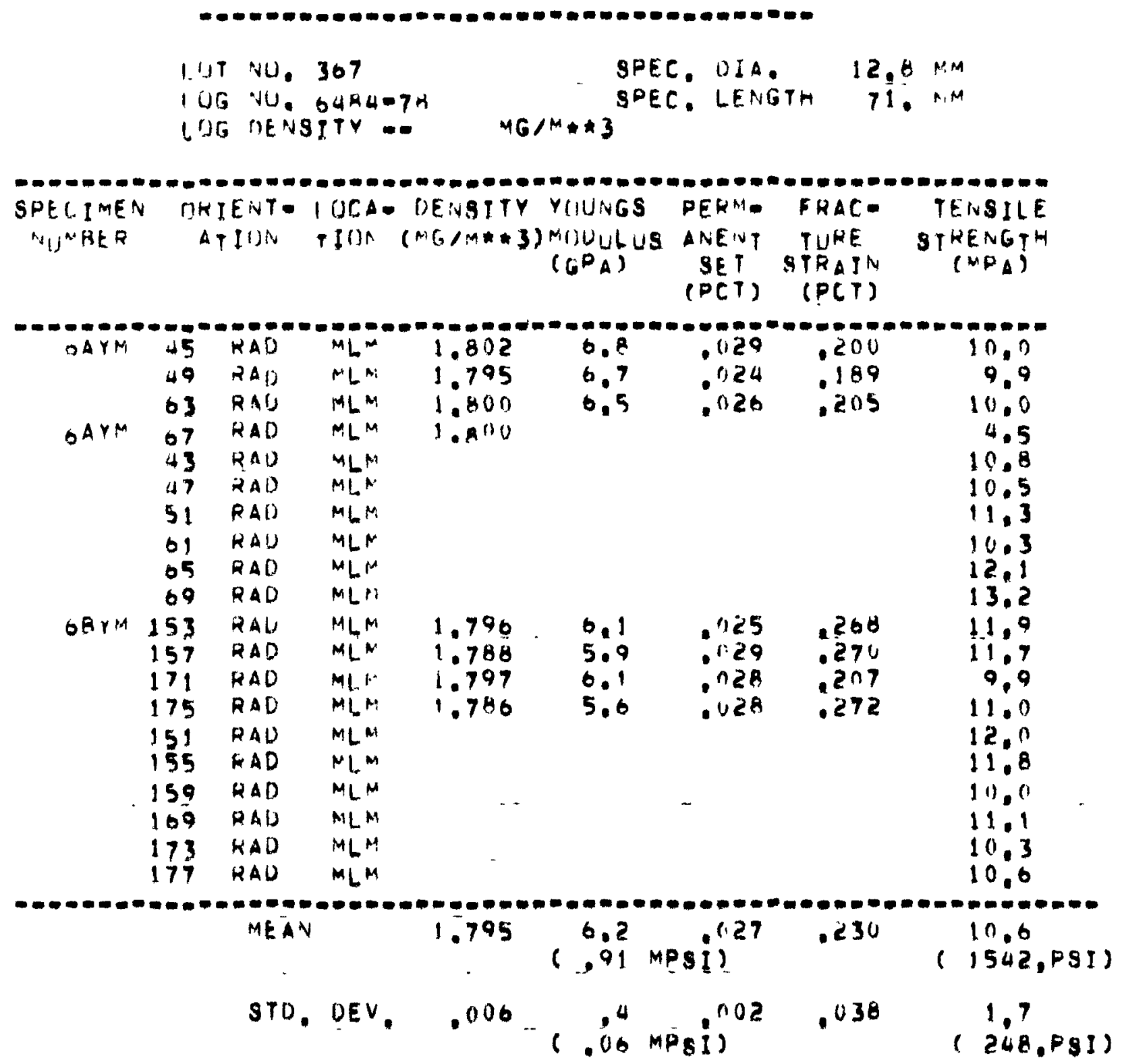


TABLE D-1 (Continued)

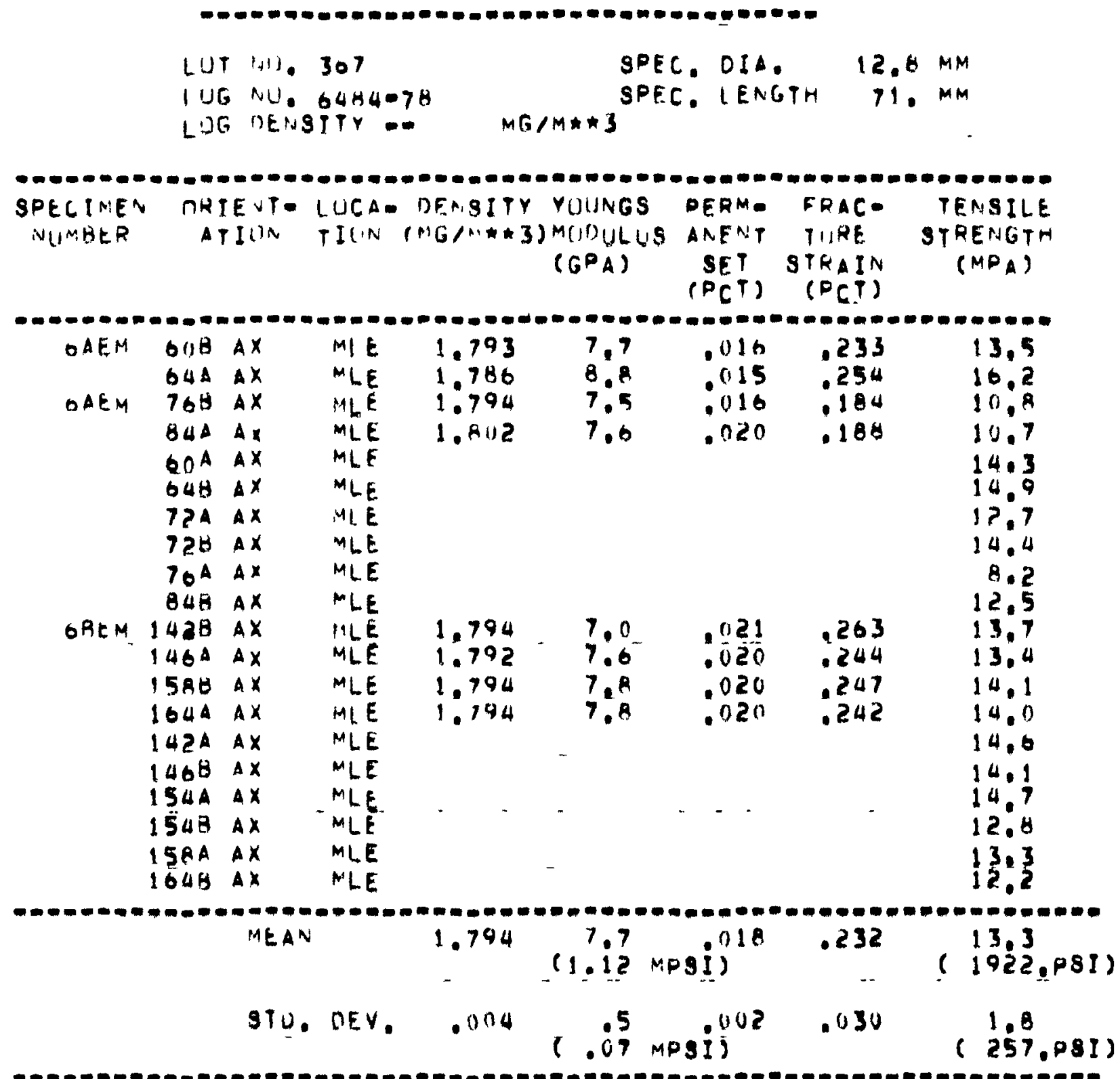


TABLE D-1 (Continued)

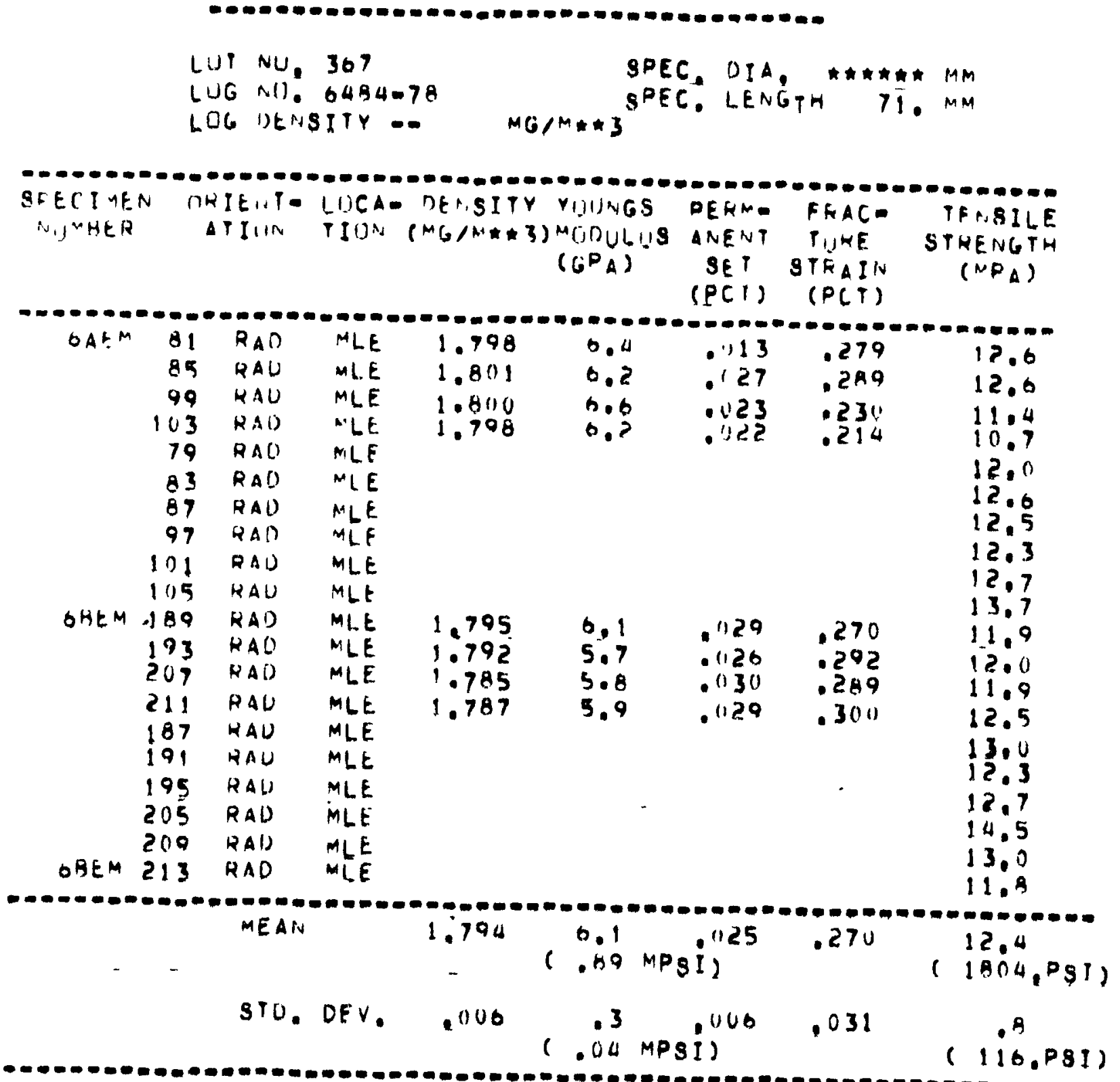


TABLE D-2

FLEXURAL PROPERTIES OF HLM GRAPHITE

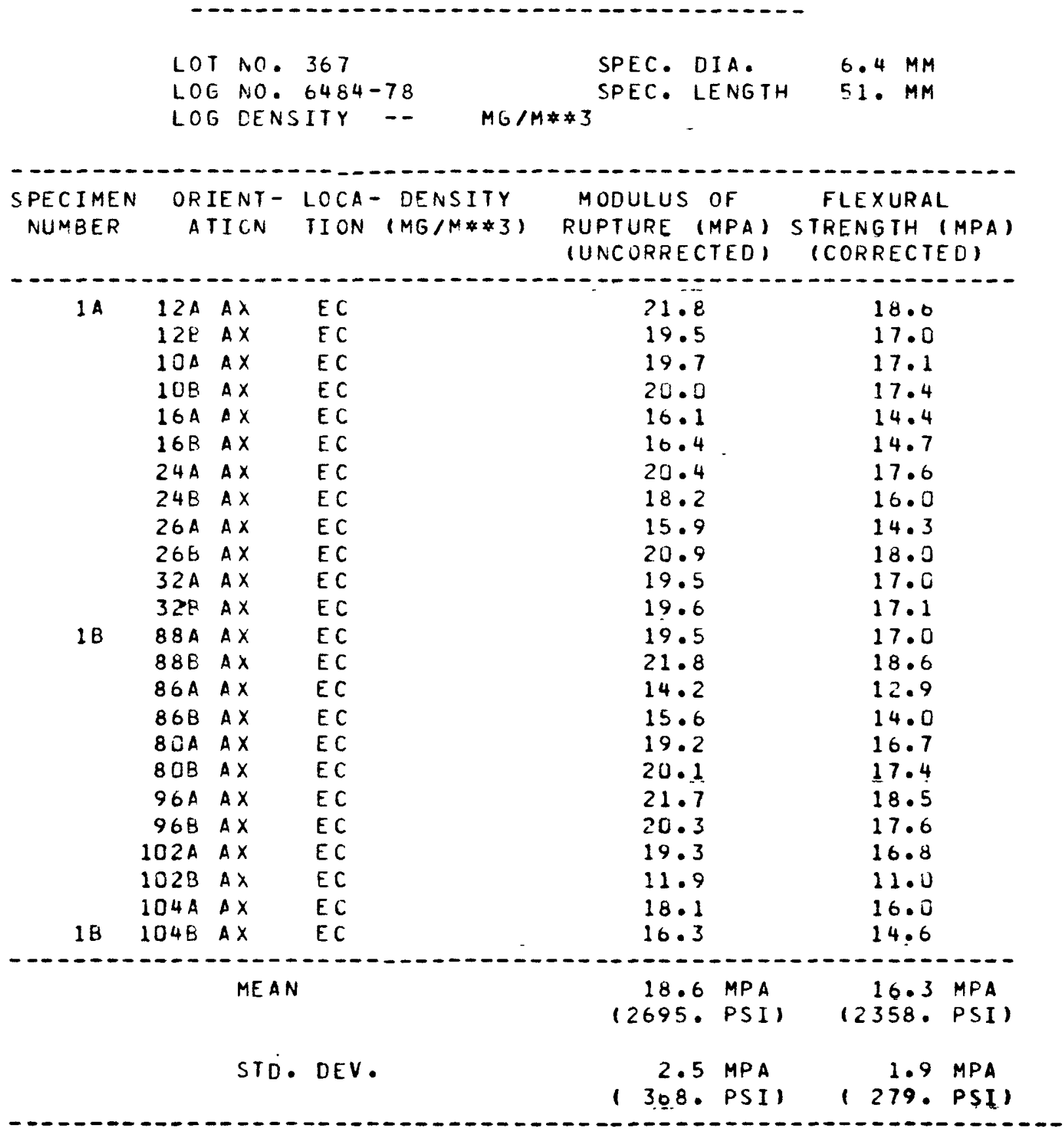




\section{TABLE D-2 (Continued)}

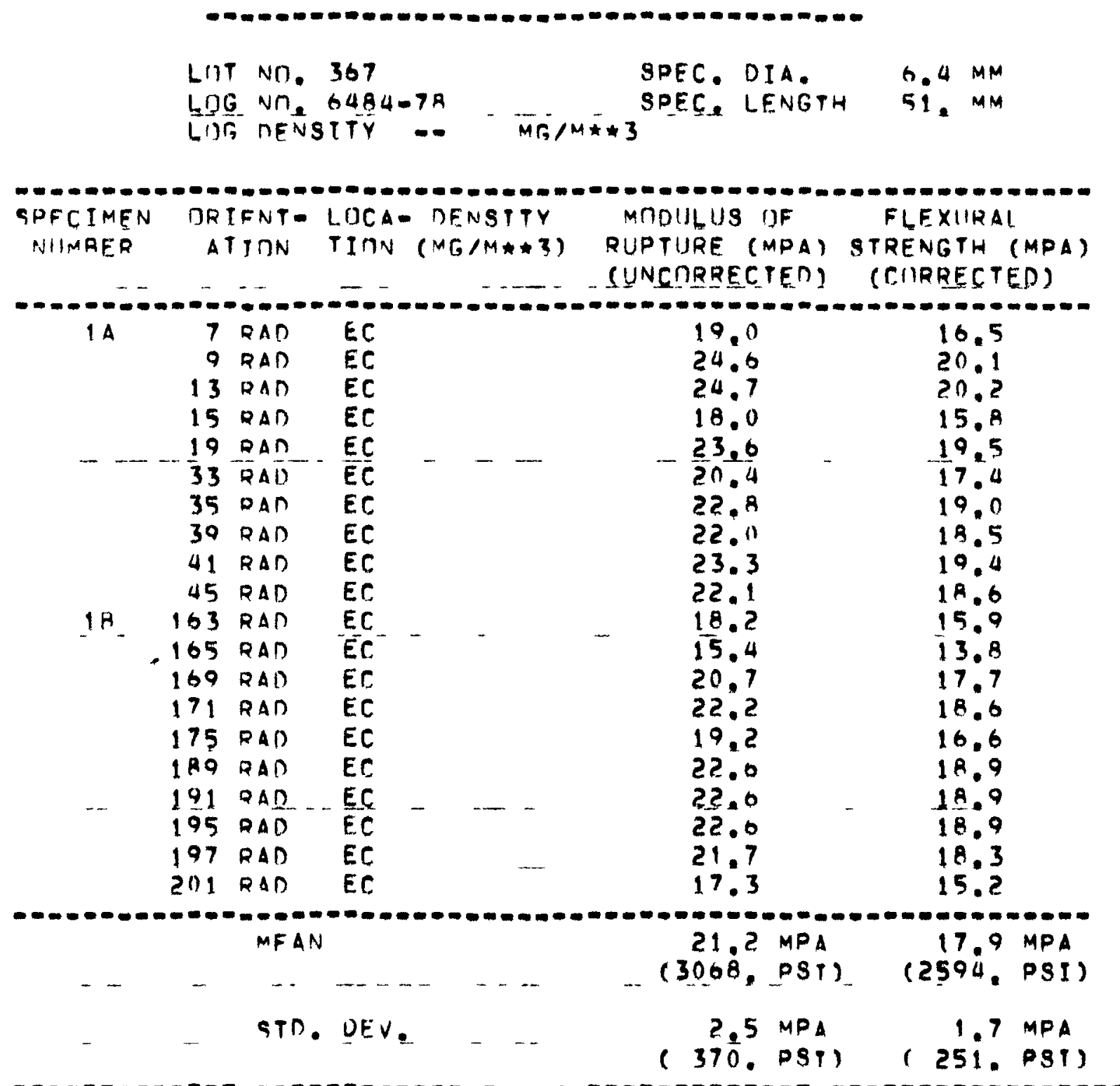


TABLE D-2 (Continued)

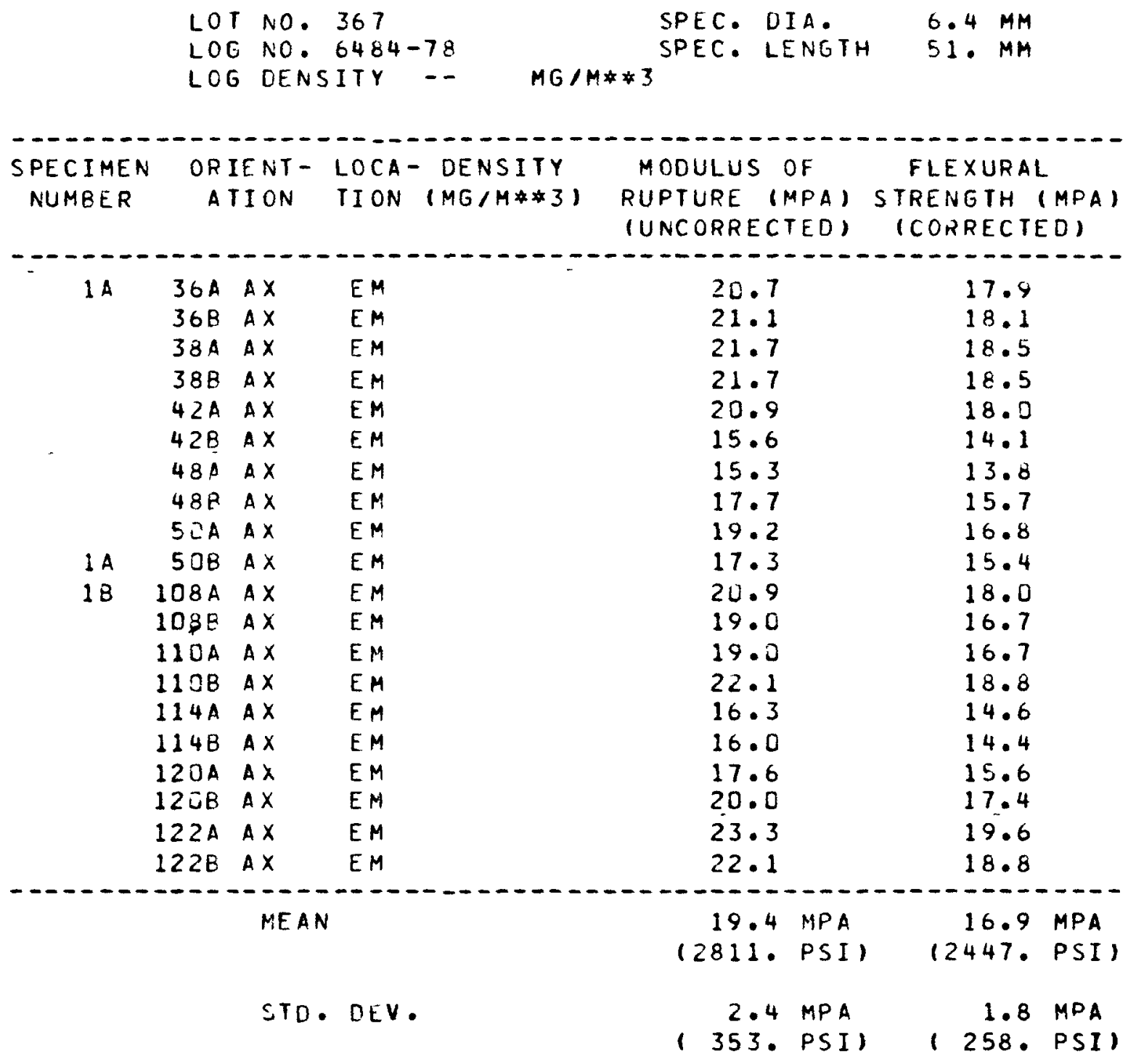


TABLE D-2 (Continued)

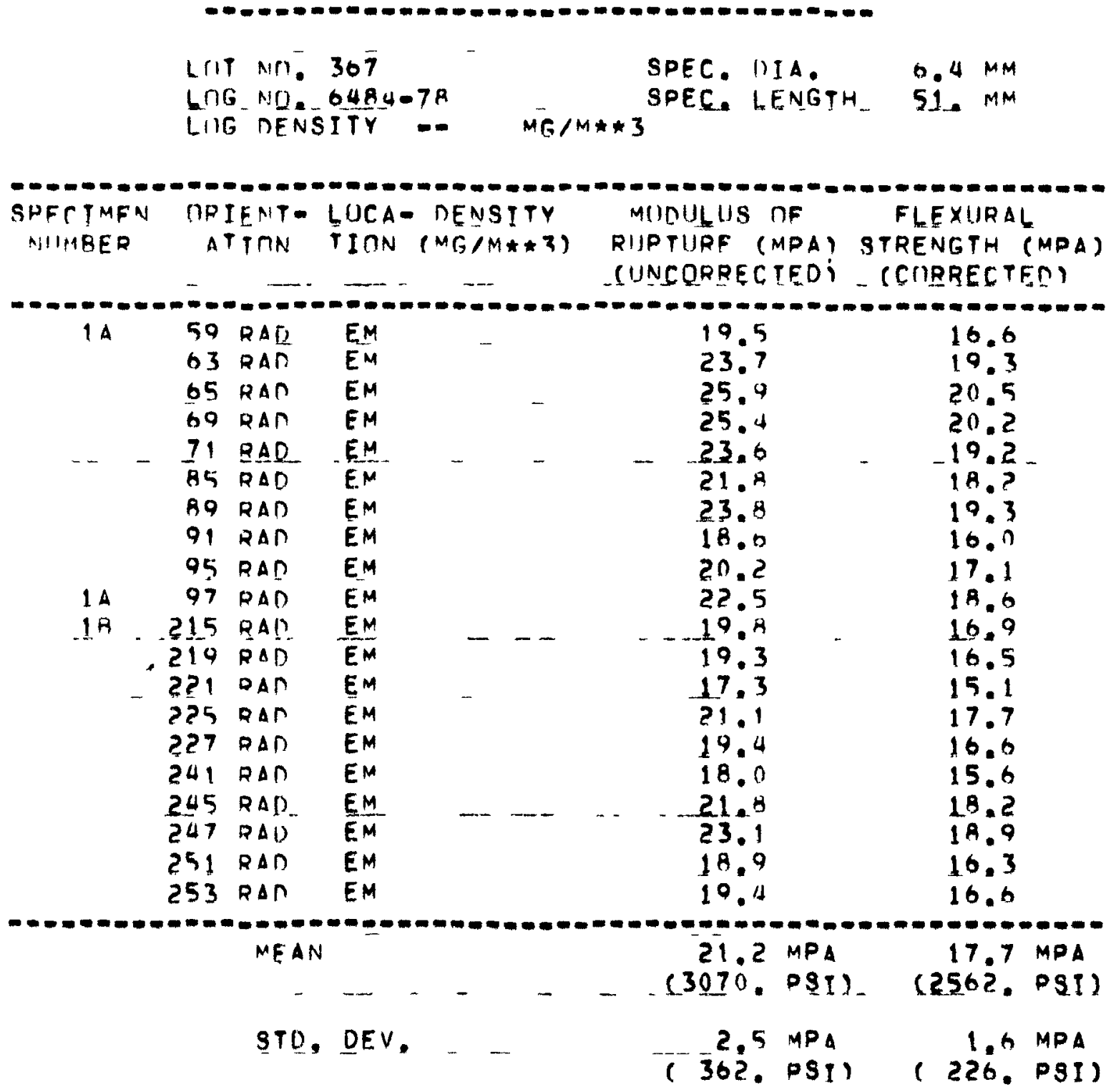


TABLE D-2 (Continued)

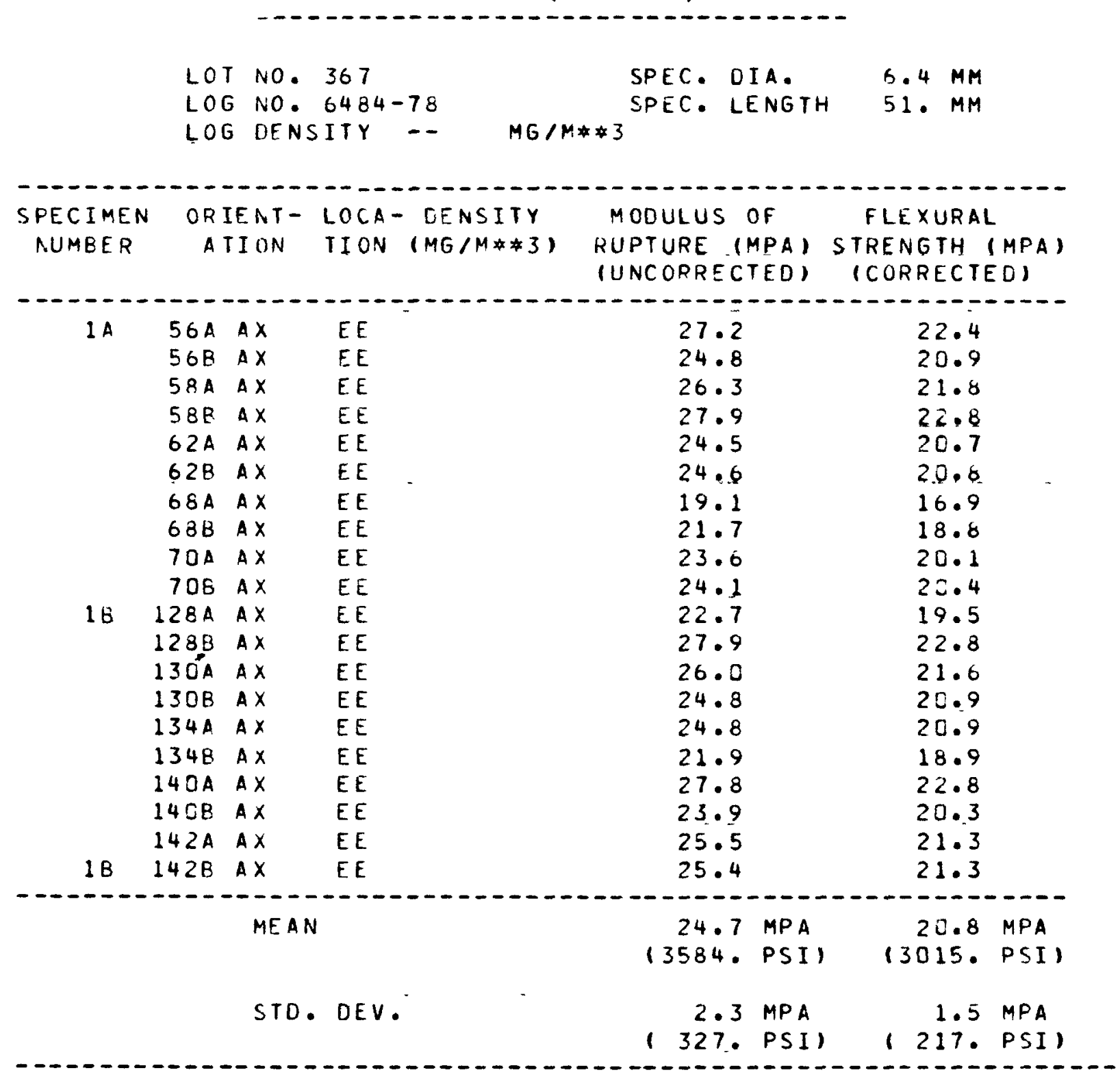


TABLE D-2 (Continued)

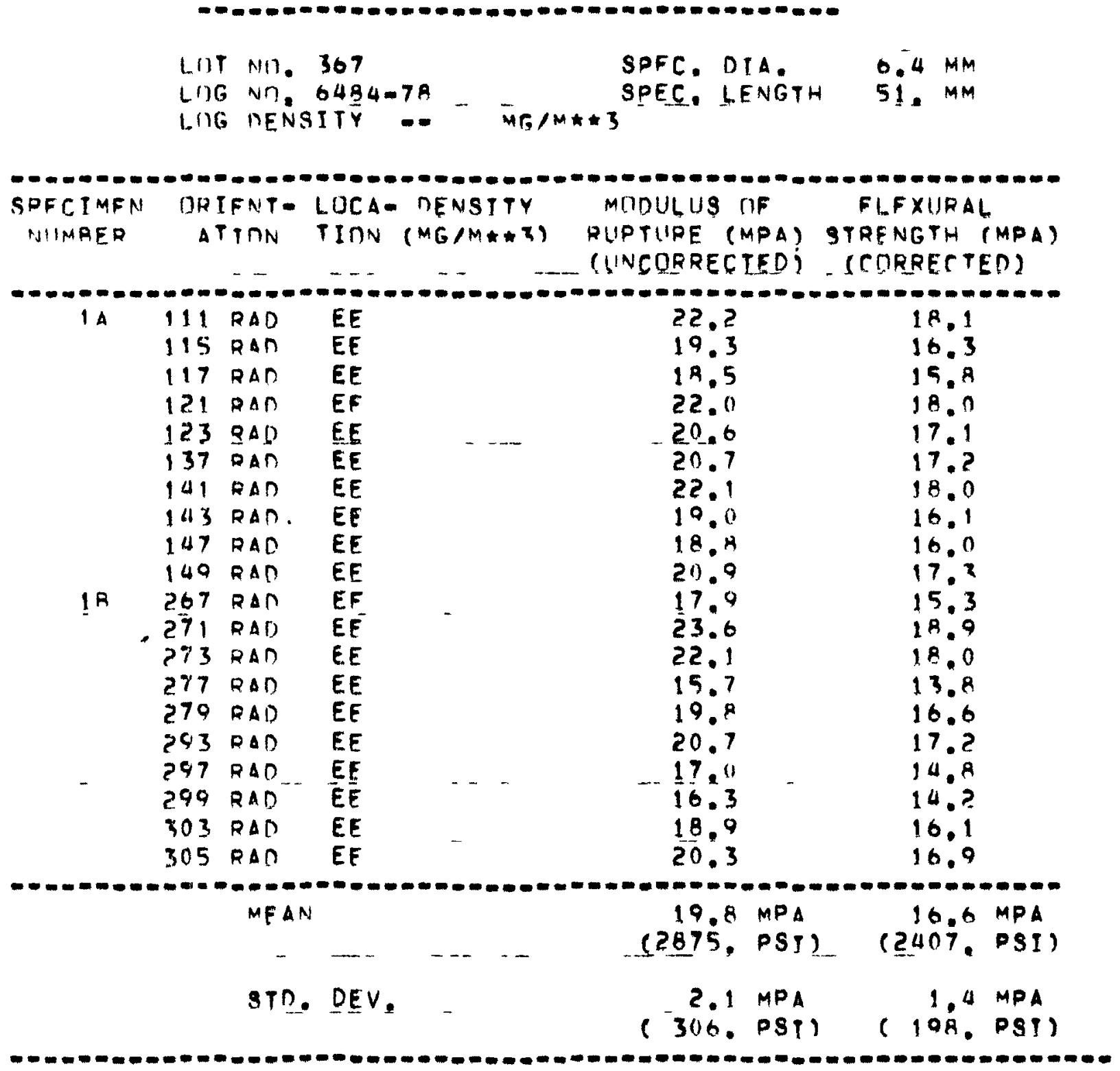


TABLE D-2 (Continued)

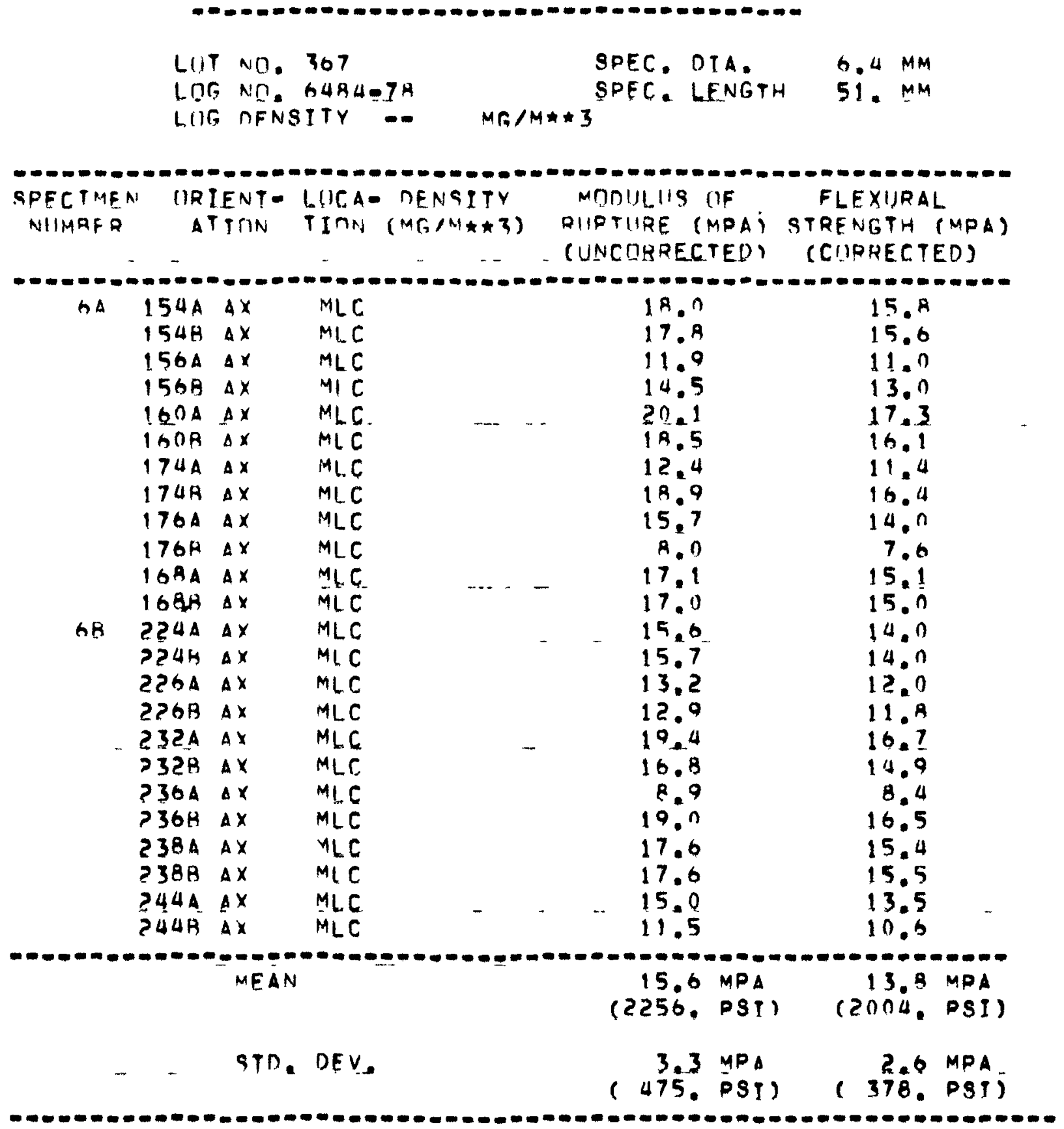


TABLE D-2 (Continued)

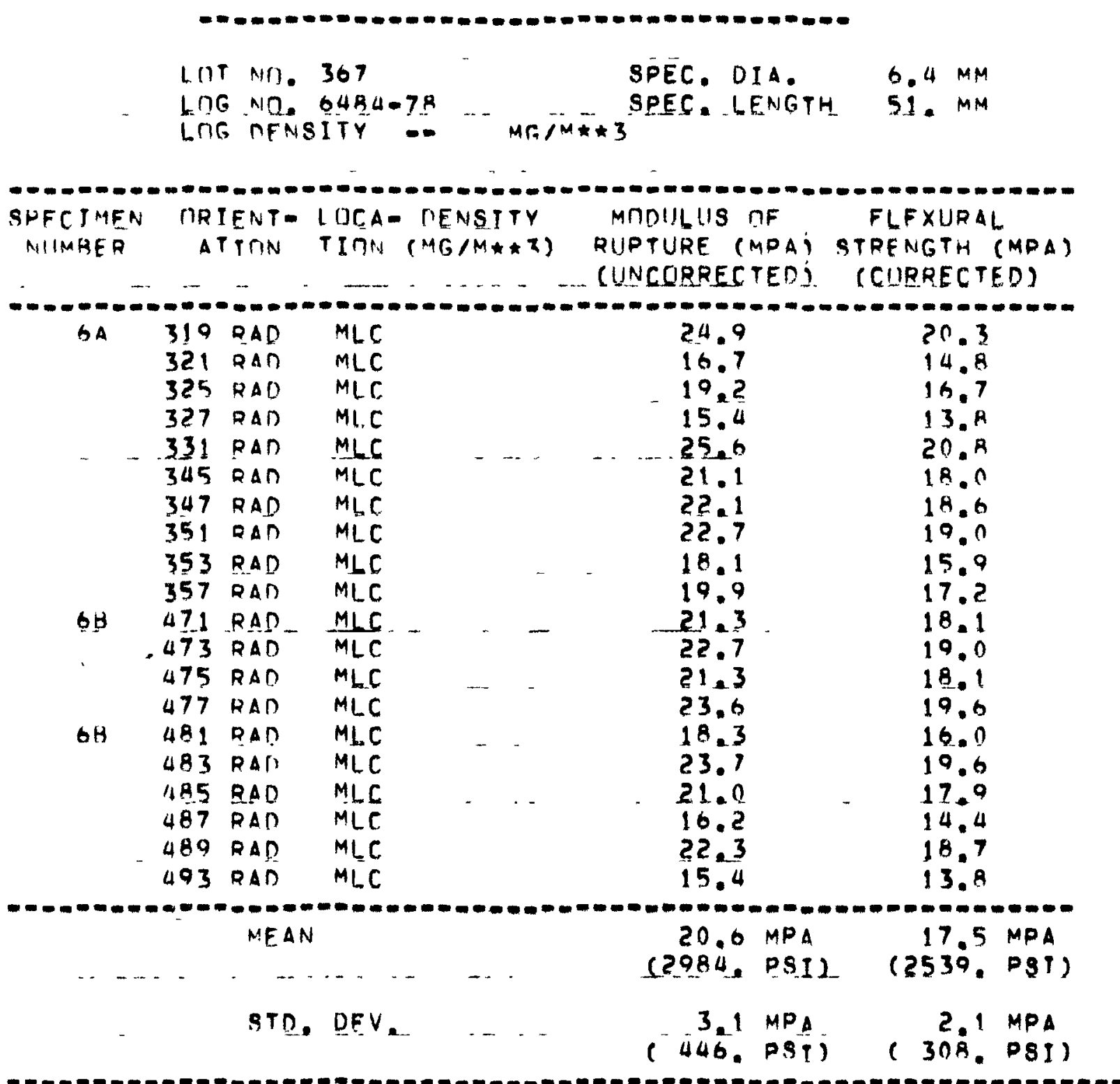


TABLE D-2 (Continued)

$\begin{array}{lll}\text { LOT NU. } 367 & \\ \text { LOR NI. 64RA-7A } & \text { SPEC. DIA. } & 6.4 \mathrm{MM} \\ \text { LOF OFASITY SPEC. LENGTH } & 51 . \mathrm{MM}\end{array}$

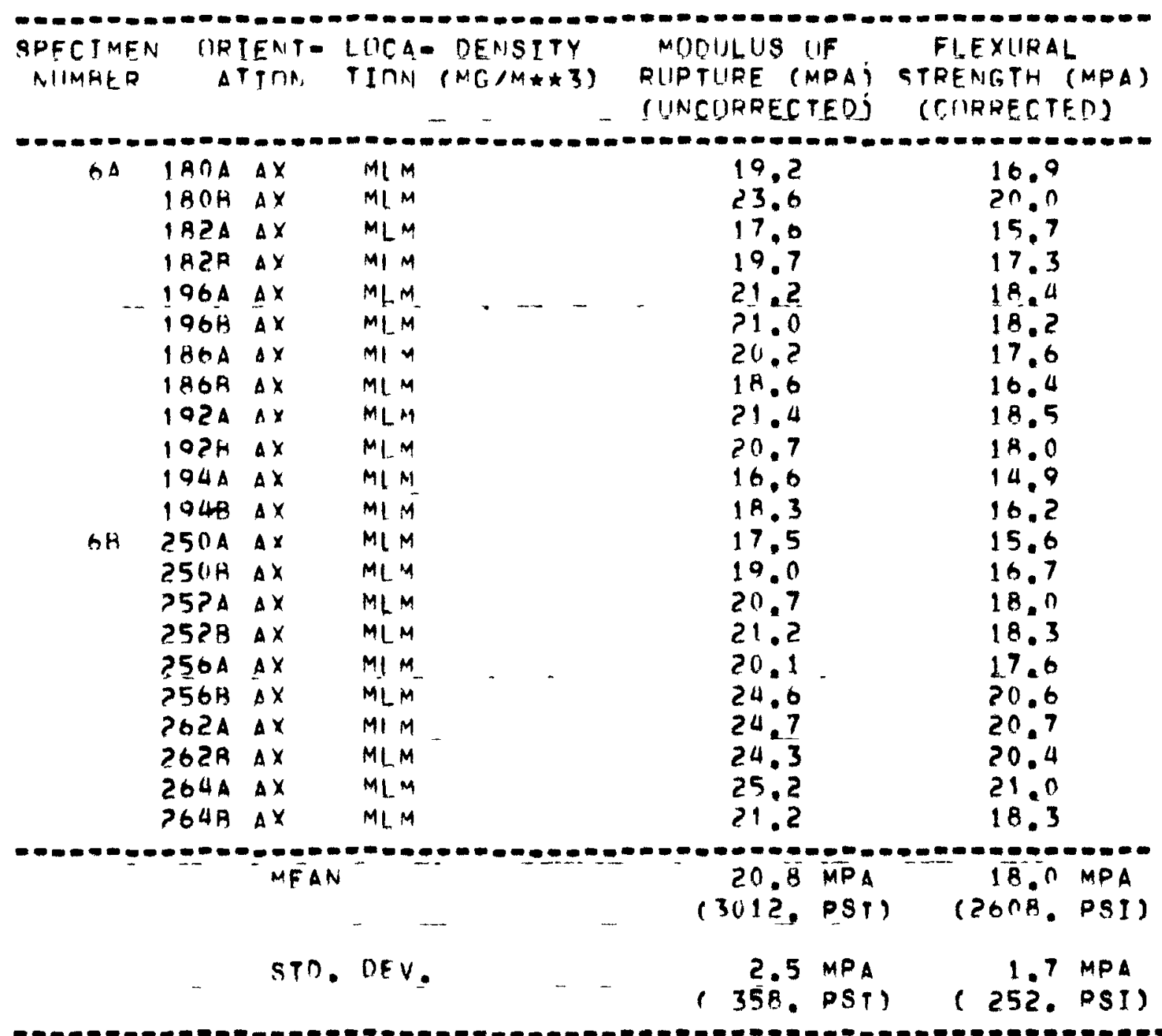


TABLE D-2 (Continued)

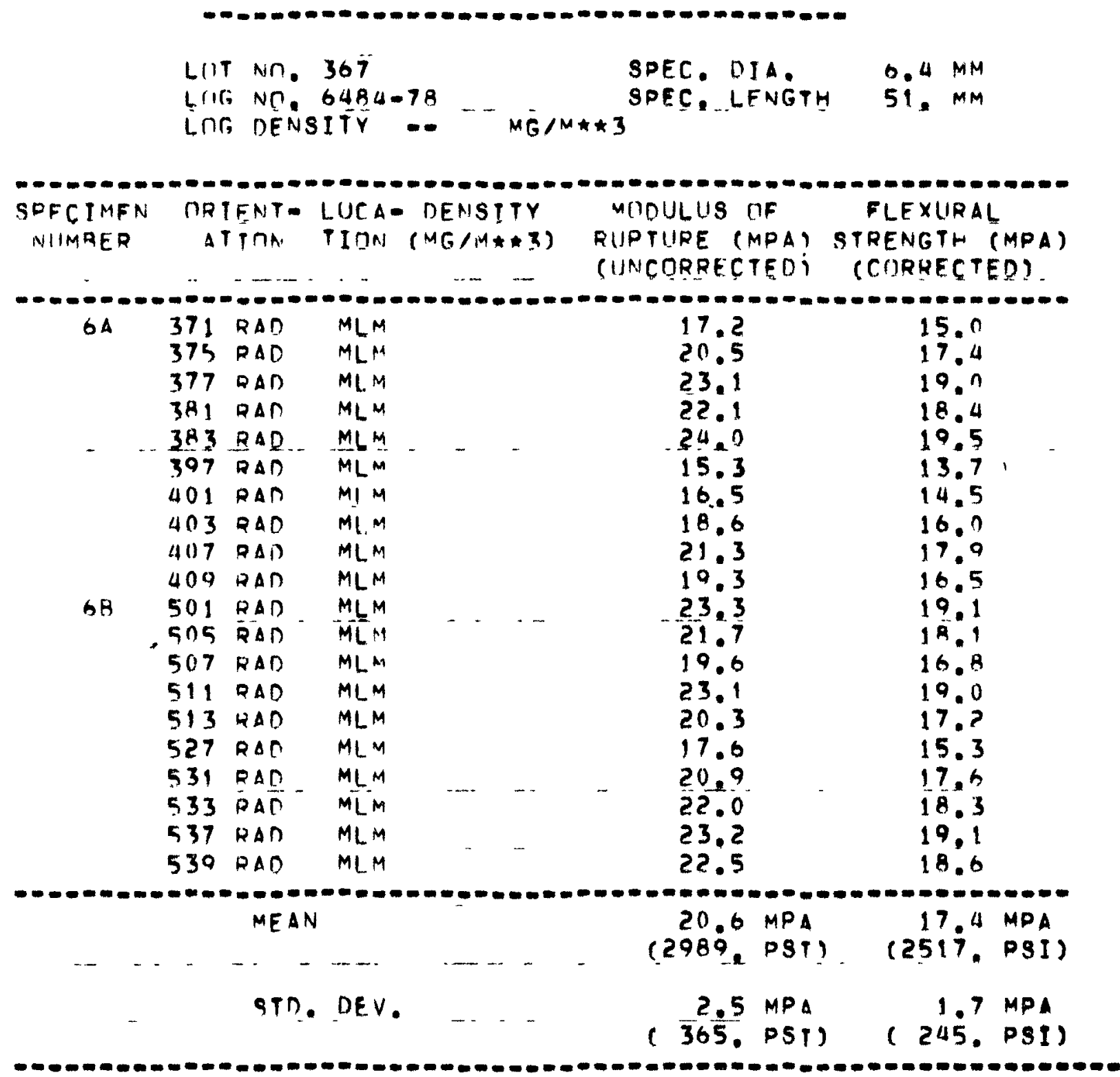


TABLE D-2 (Continued)

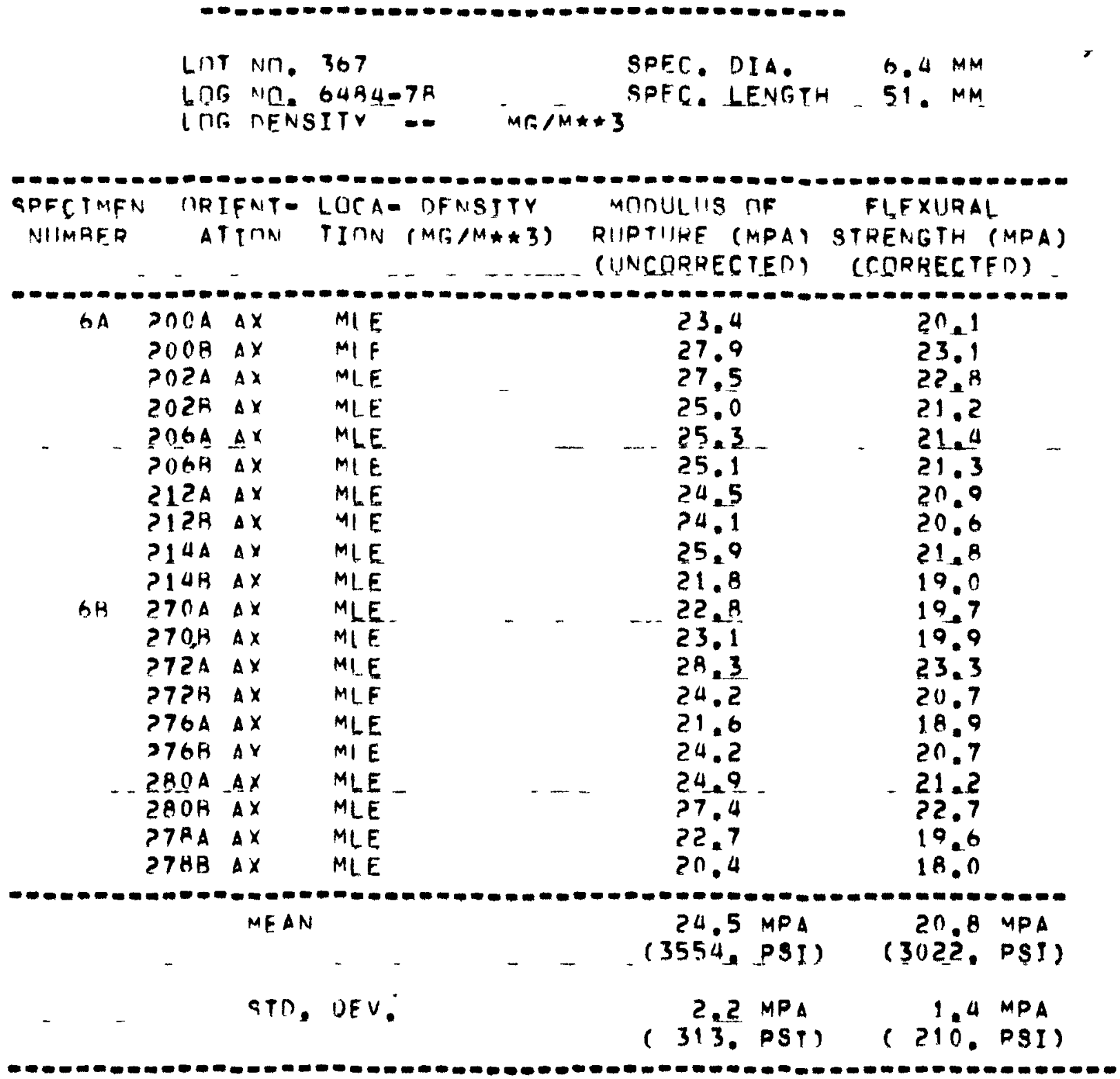


TABLE D-2 (Continued)

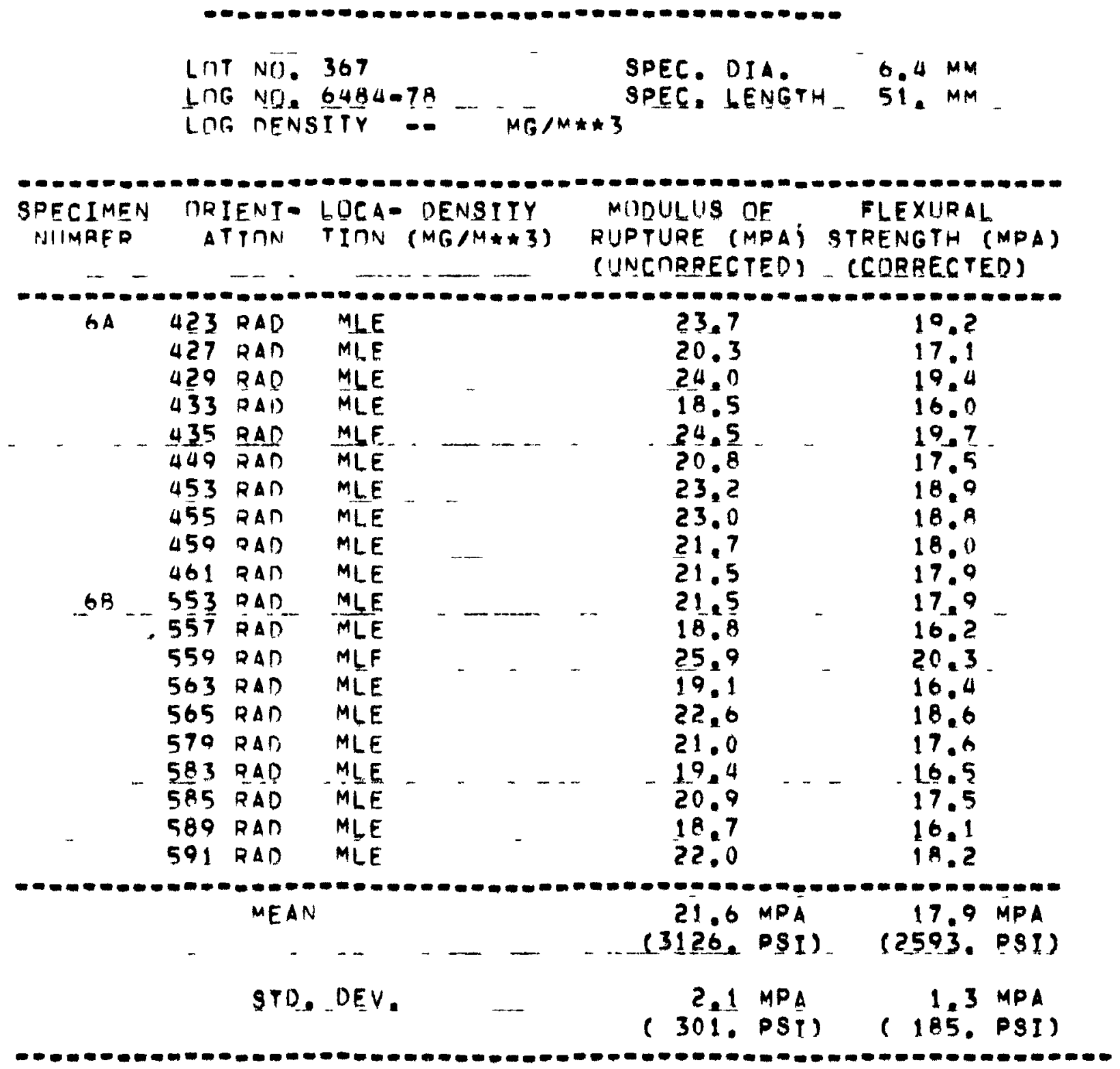


TABLE D-3

COMPRESSIVE PROPERTIES OF HLM GRAPHITE

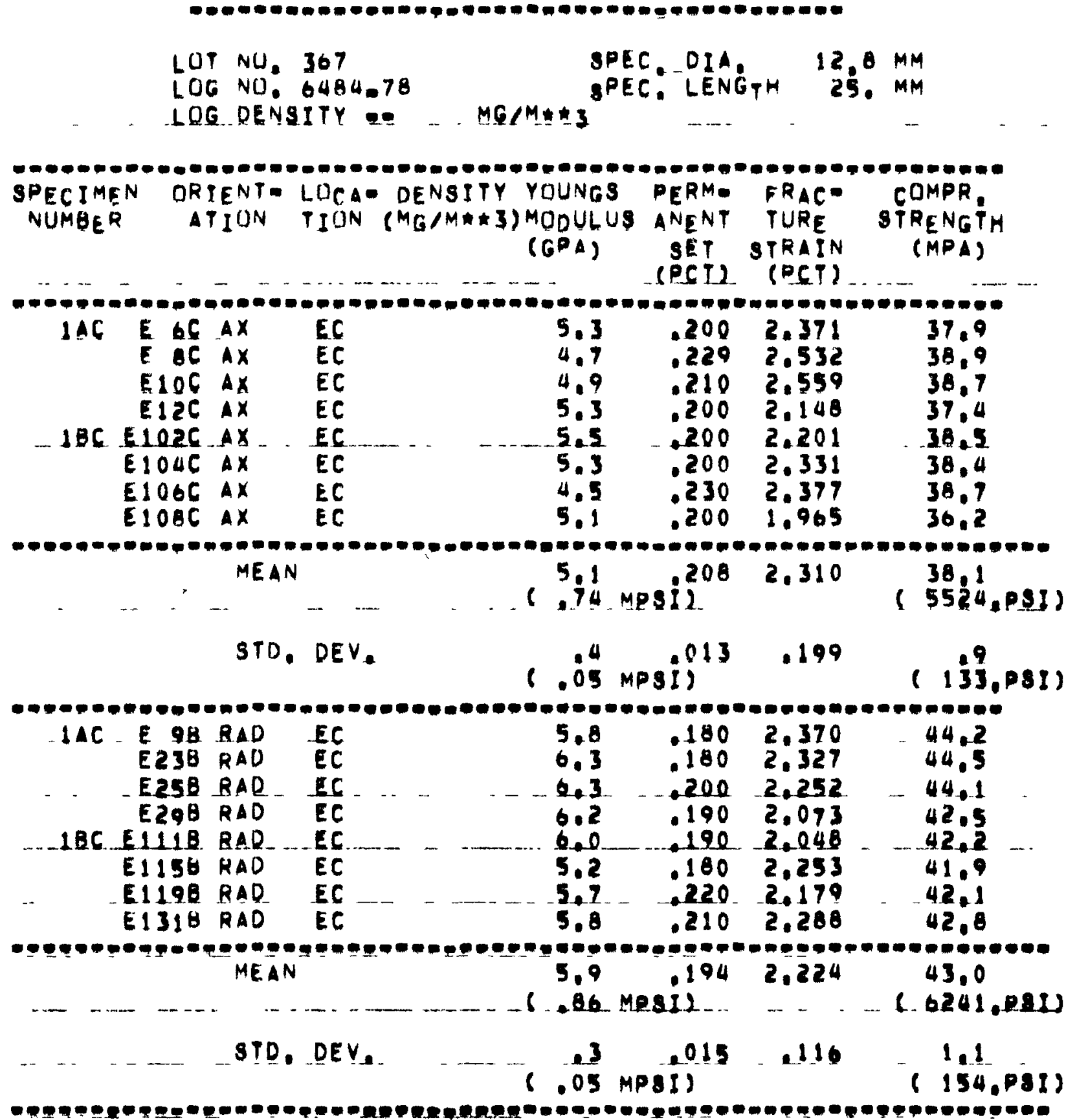


TABLE D-3 (Continued)

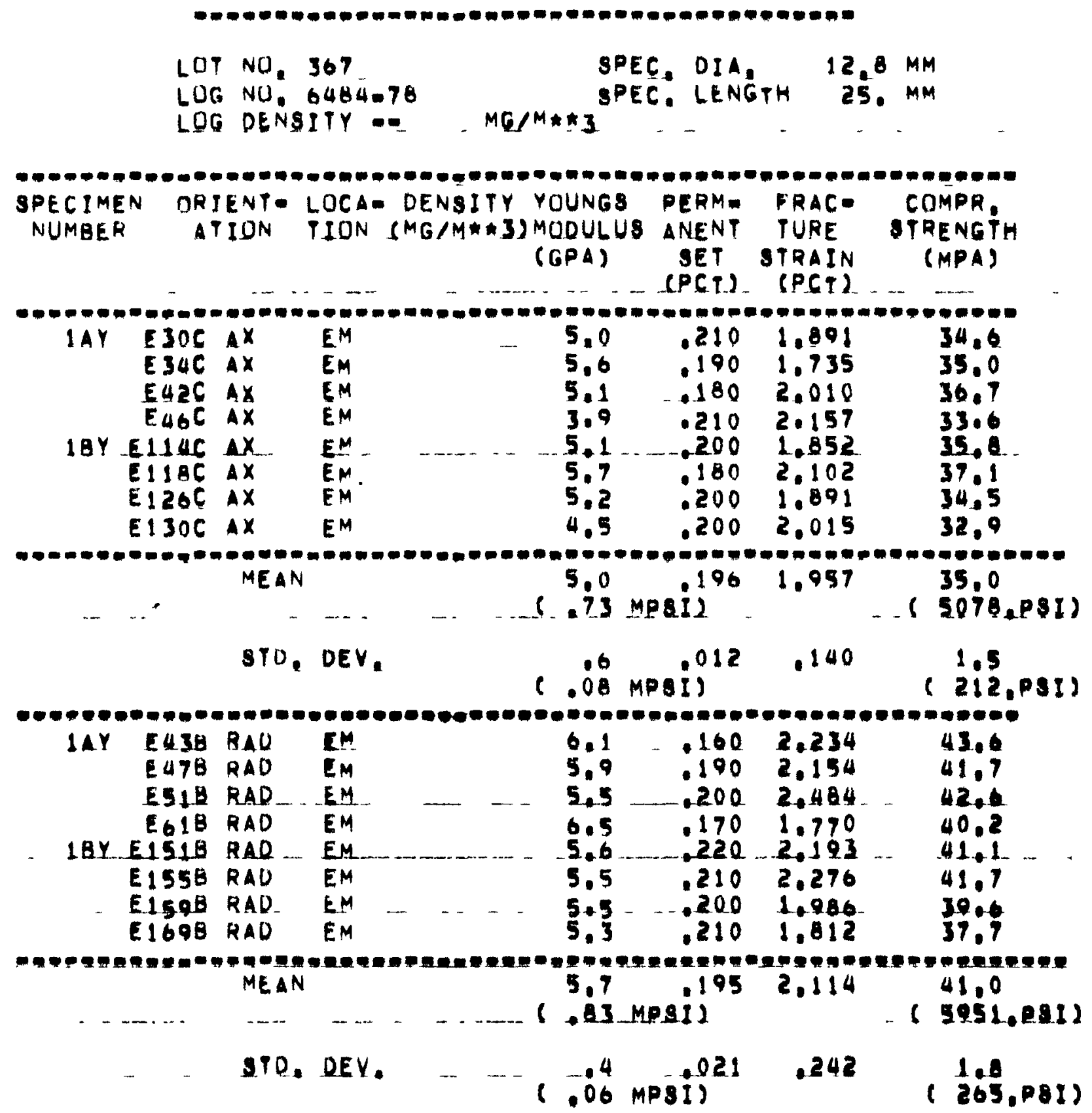

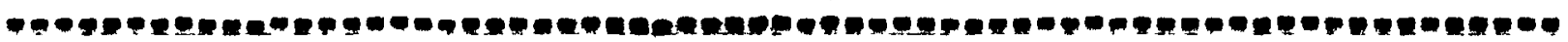


TABLE D-3 (Continued)

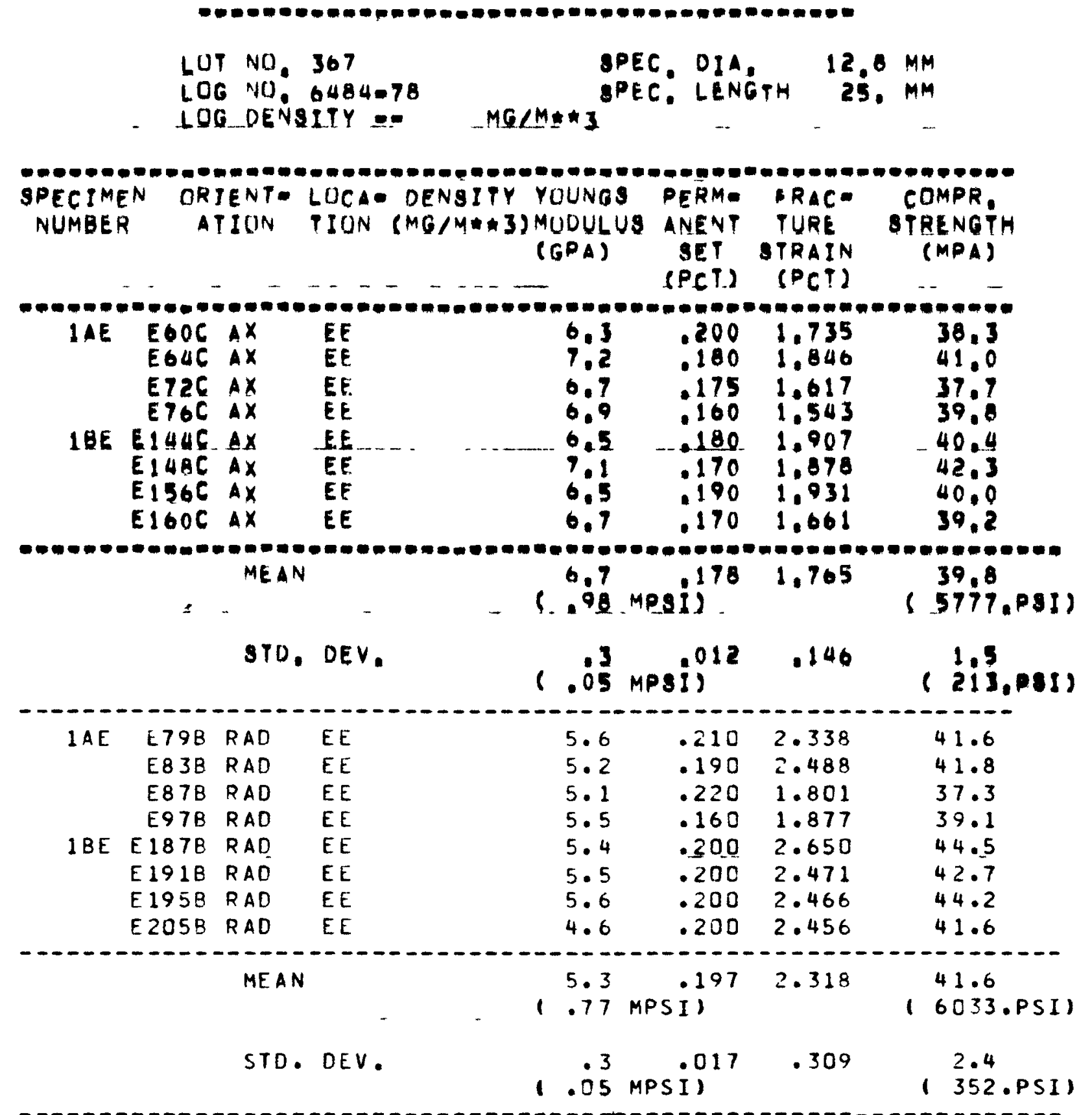


TABLE D-3 (Continued)

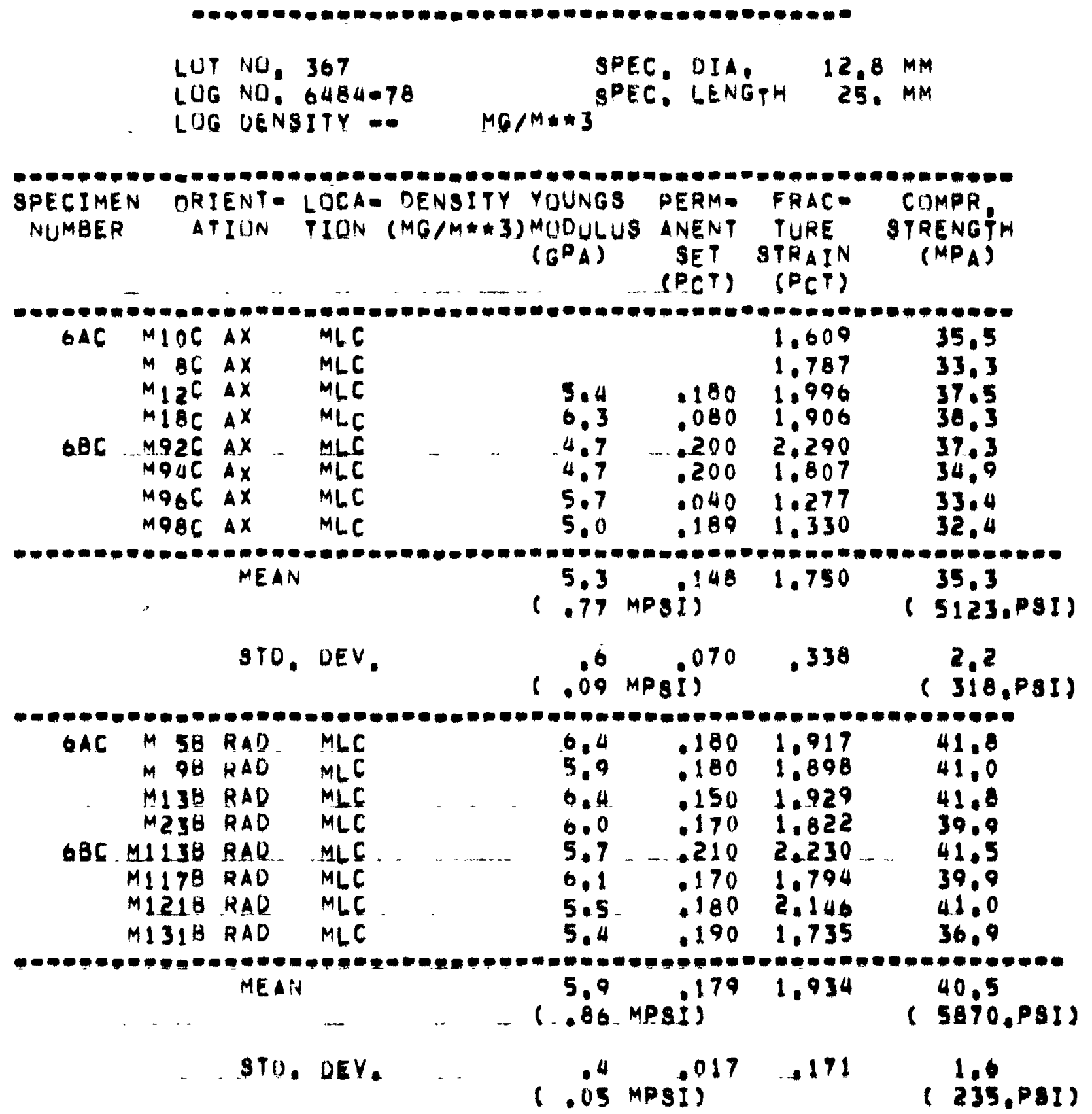

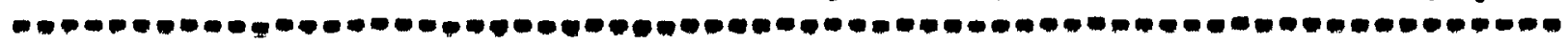


TABLE D-3 (Continued)

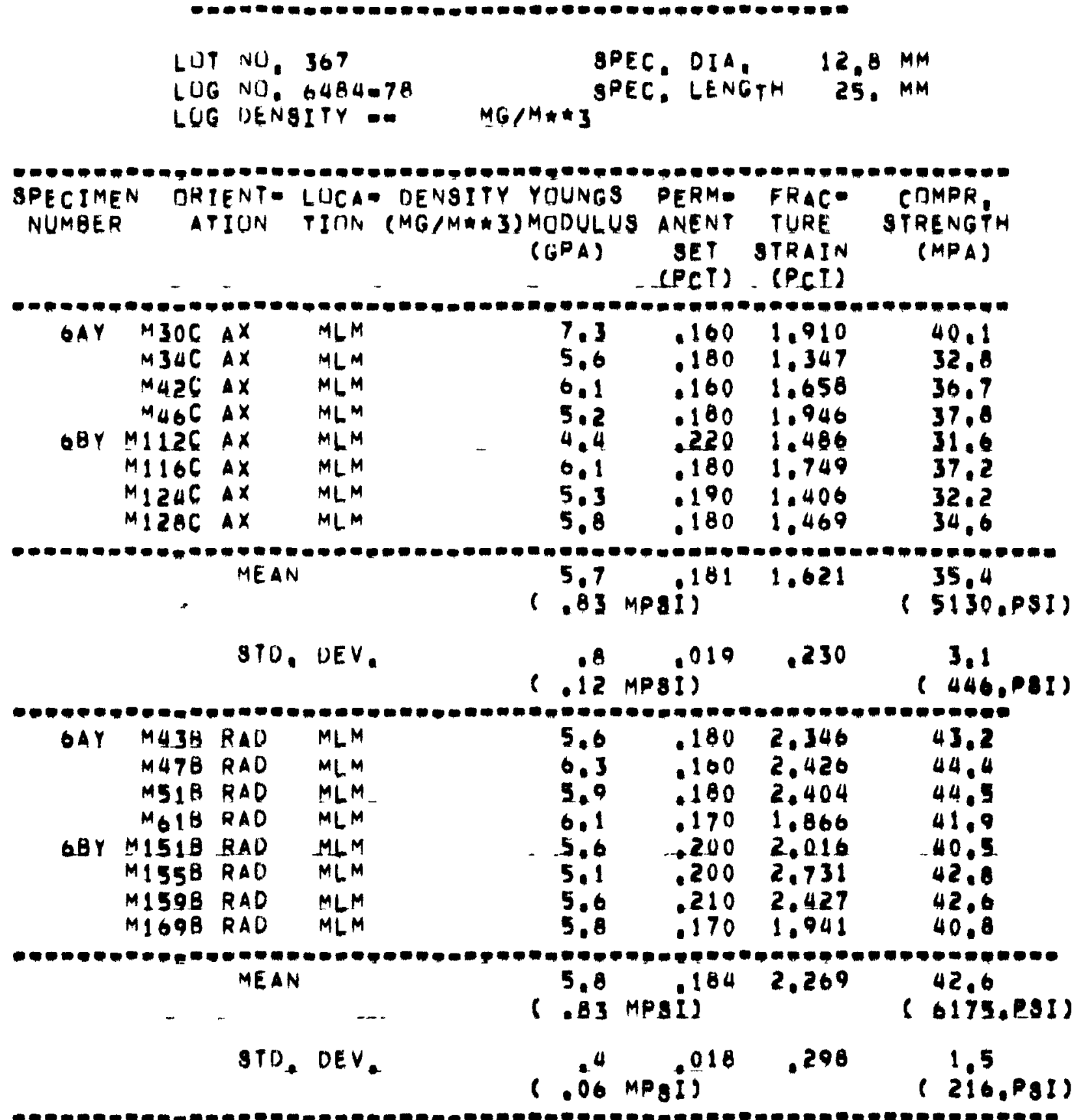


TABLE D-3 (Continued)

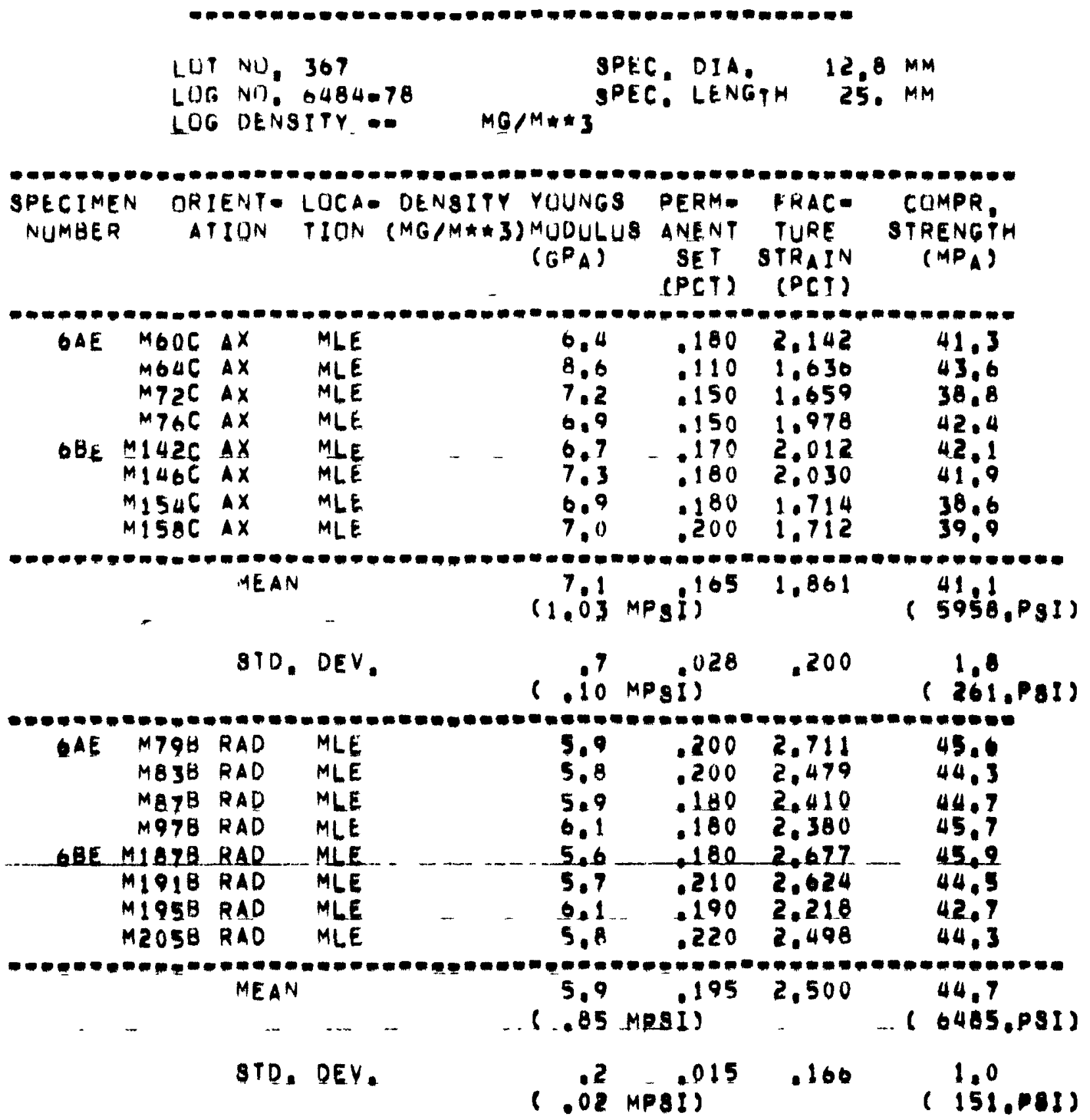

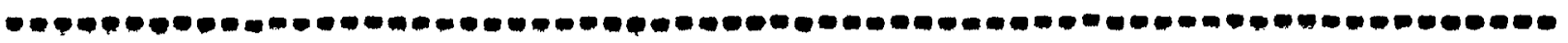


TABLE D-4

OF HIM GRAPHITE

LOG NO. 6484-78, SPECTROGRAPHIC ANALYSIS

LAB, NU, 44247 REPURI ILII G ENGLE

SAMPLEL GRAPAIIE QUB4-76-ETOO PROJECT NU, 1224146005

DILUTLONI

1.0400

DATEL $0.22 \cdot 70$

ELEMENT CONGENTRATION PPN

ELEMENT CONCENTRATIUN, PDM

PLATE NU, 100006
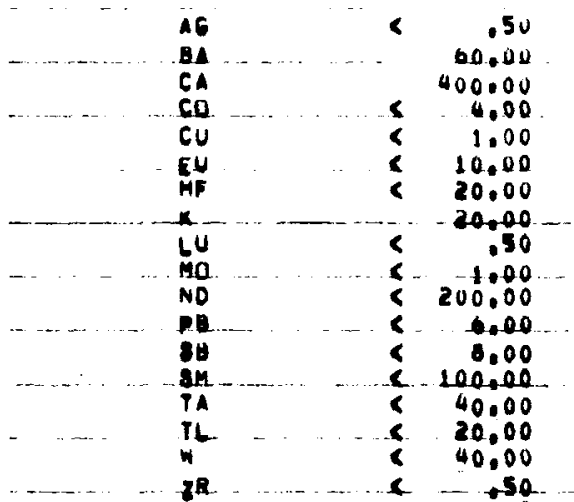

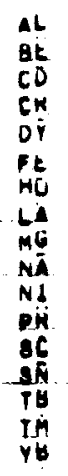

$\quad 0.00$
$<\quad .50$
10.00
10.00
20.000
1000.00
10.00
10.00
2.00
10.00
20.00
$<\quad 100.00$
1.00
$<\quad 6.00$
40.00
4.000
$<\quad 10.00$

ELEMENI

CONGEOTRAIION, PPM

GAB, NO. 44247 $<\ldots$

re$$
10.00
$$

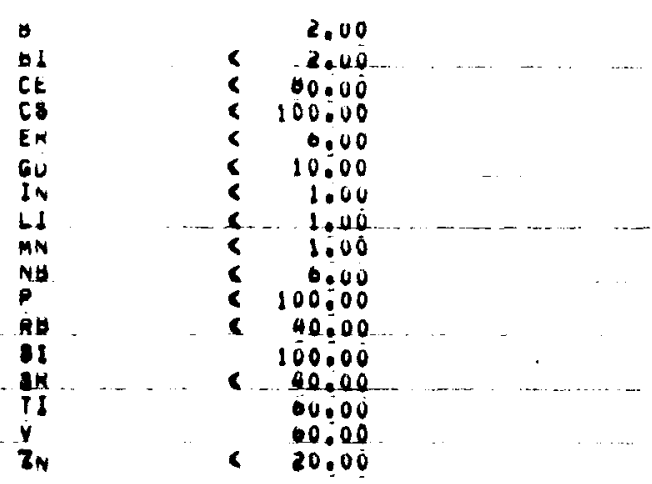

DATEI OBO21076

PLAFE NO. $16=066$

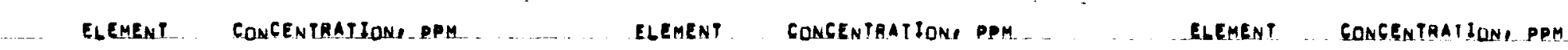

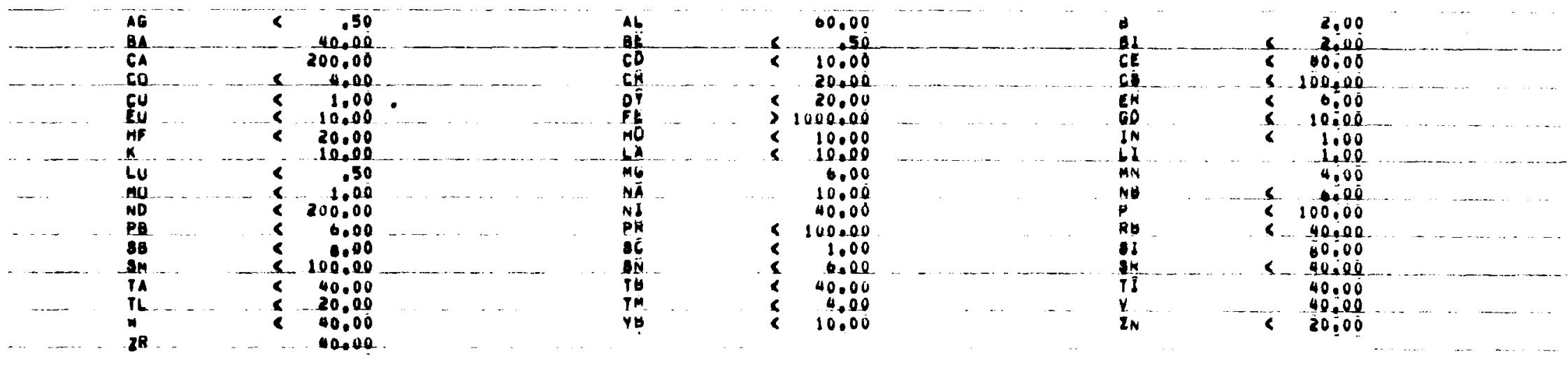

CONCENTAAIION GASED ON URGINAL SAMPLE BEFOAE OILUTION WITH DILUENT

2 MEANG GREATEK THAN

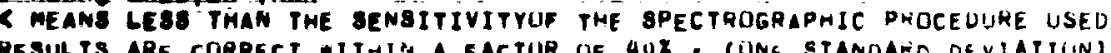




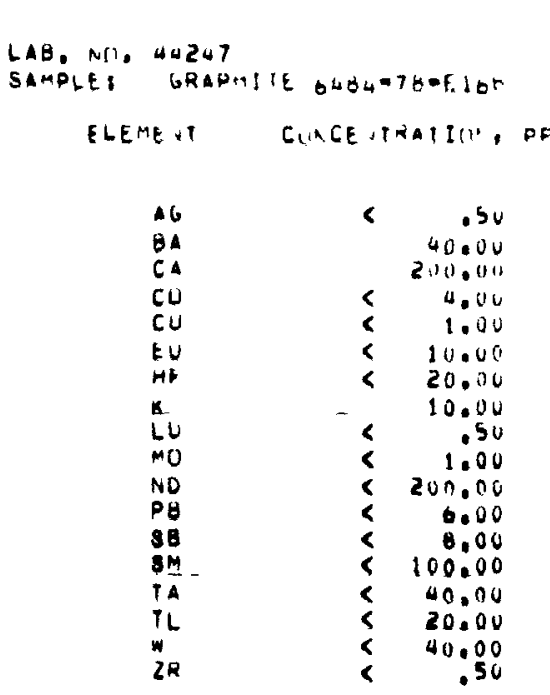

LAB, NO, 44247

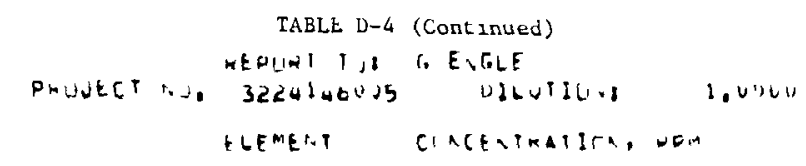

$A L$
$O E$
$C U$
$C K$
$D Y$
$P E$
$M U$
$L A$
$L G$
$N A$
$N I$
$P H$
$8 C$
$8 N$
IO
IM
$Y U$

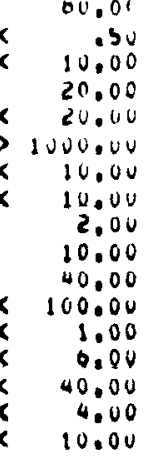

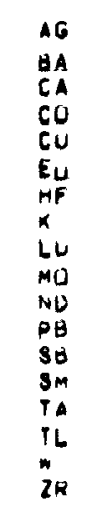$$
\text { CONCENTRATION, PPM }
$$

PRUJECI NO, REPURI TOI 6 ENGLE

ELEMENT

CUNCENTRATION, DPM

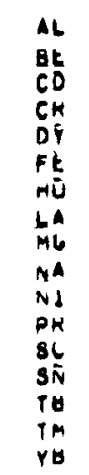

$A L$
$B E$
$C D$
$C K$
$D Y$
$F E$
$M U$
$L A$
$M G$
$M A$
$N I$
$P K$
$8 L$
$S N$
$T U$
$I M$
$Y B$

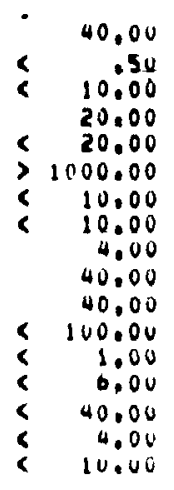

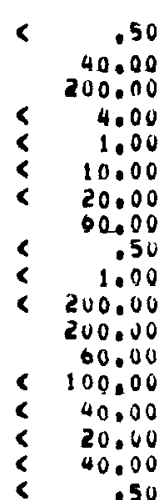

0,000

.00

0.00

4.00
0.00
Lalfi $0-21=10$ blate N. To-vo

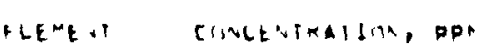

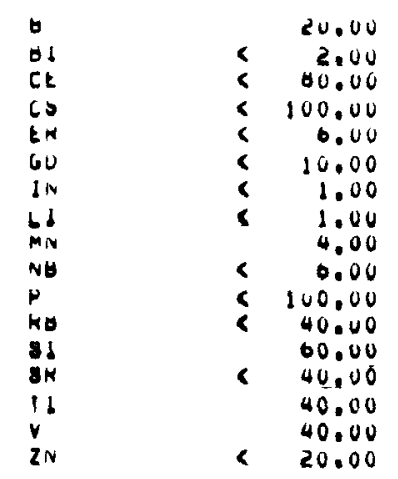

DAlES $0-21=70$

PLAJE ND, 760060

ELEMENT CONCENTHATIJNA PPM
$1.000 y$

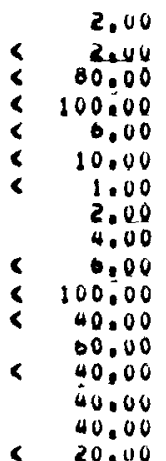




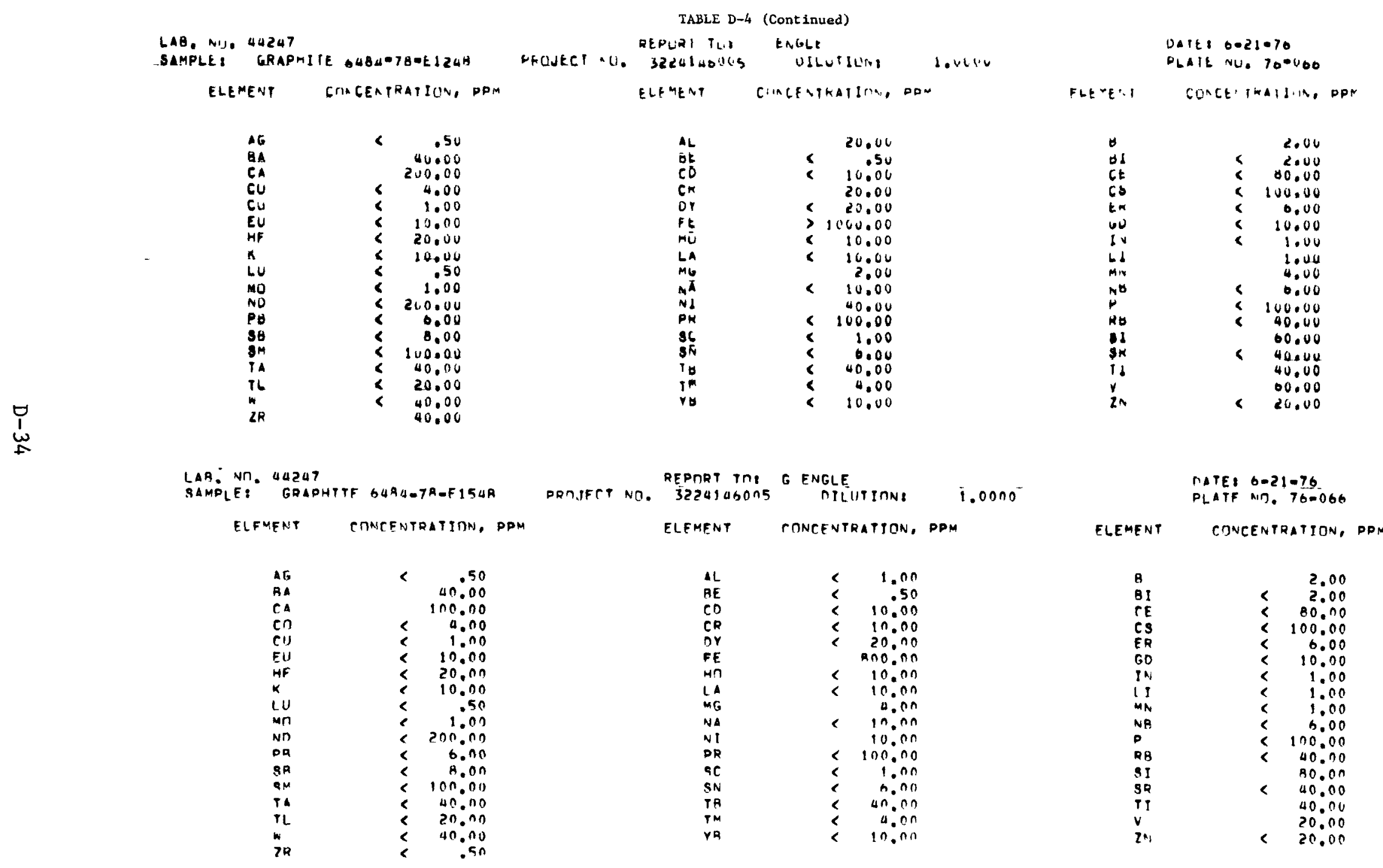

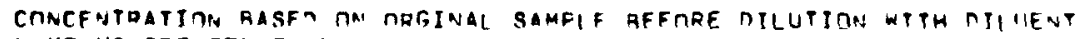
T MEANS GREATEQ TWAM

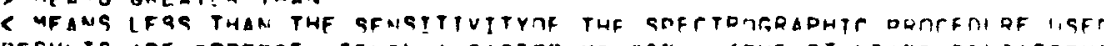

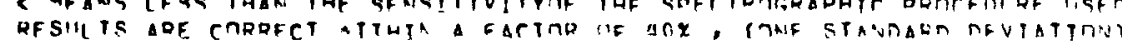




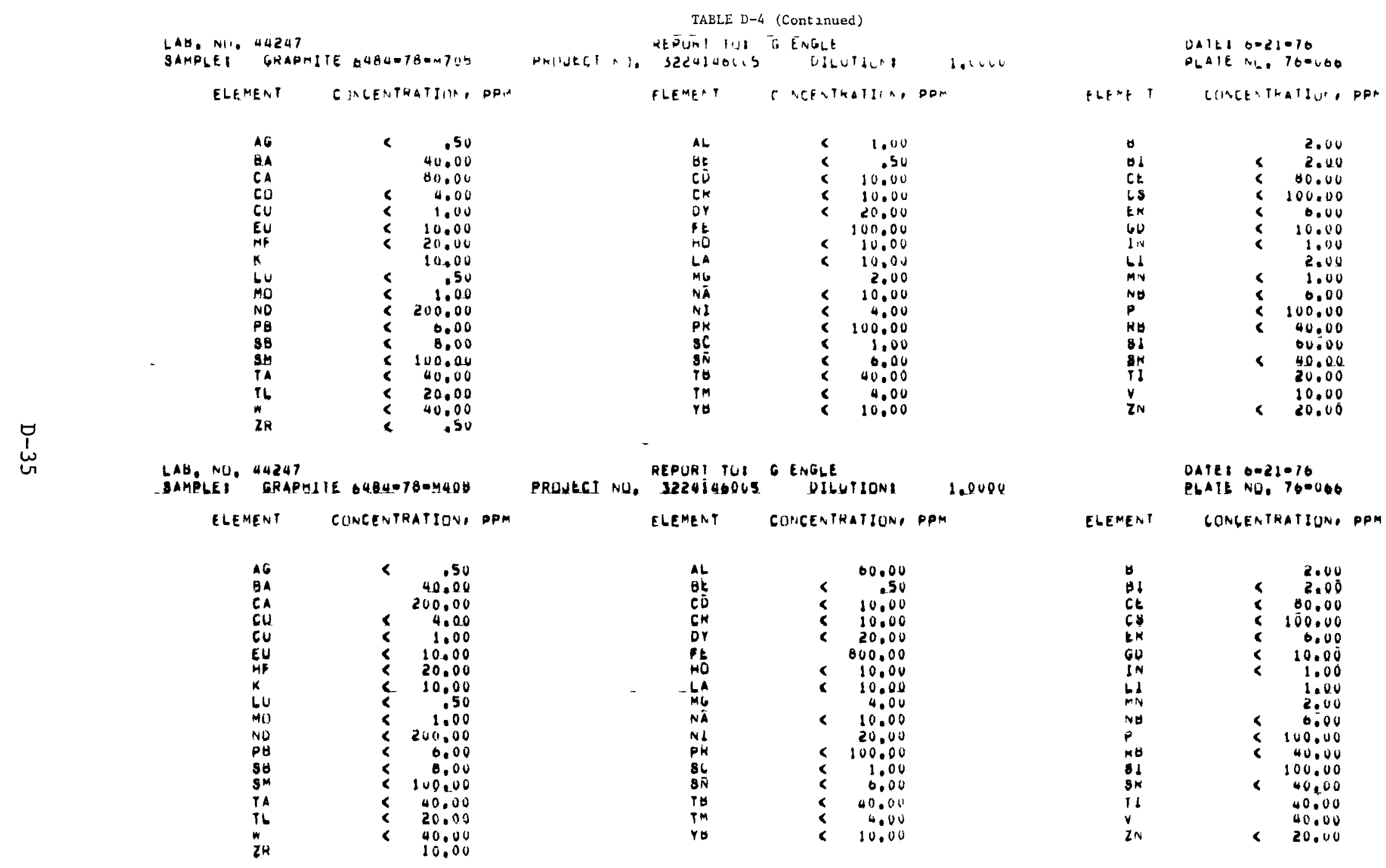

CONCENTRATION GASED UN ORGINAL SAMPLF BEFURE DILUTION WITM DILUENI

2 MEARS GREAIEK THAN

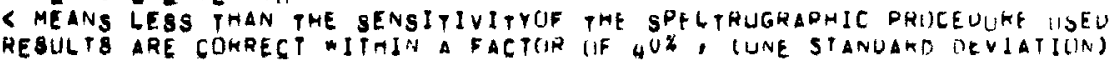




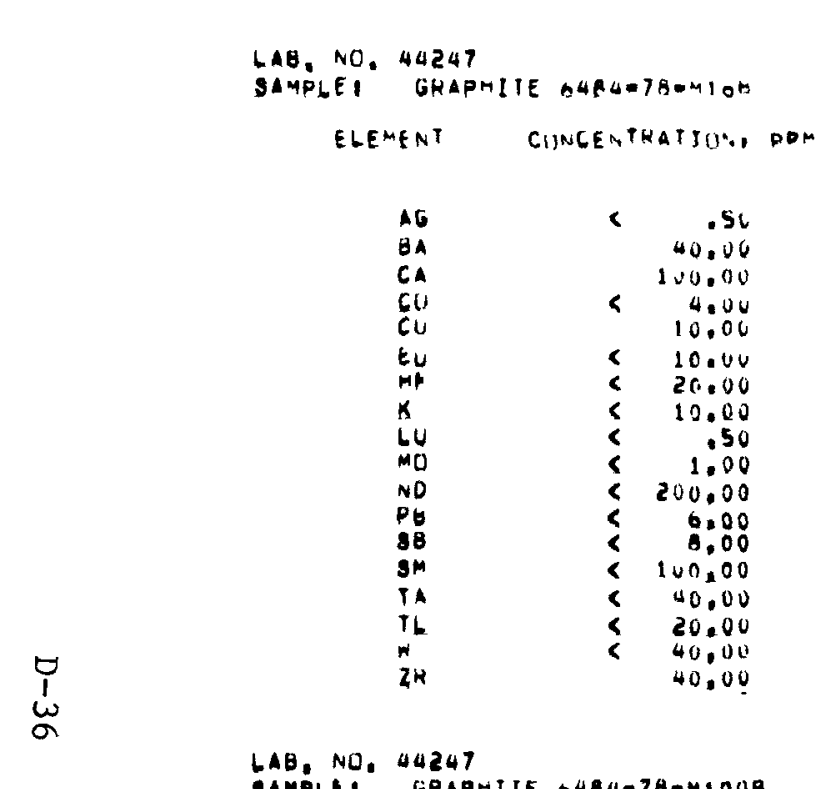

TABLE D-4 (Continued)
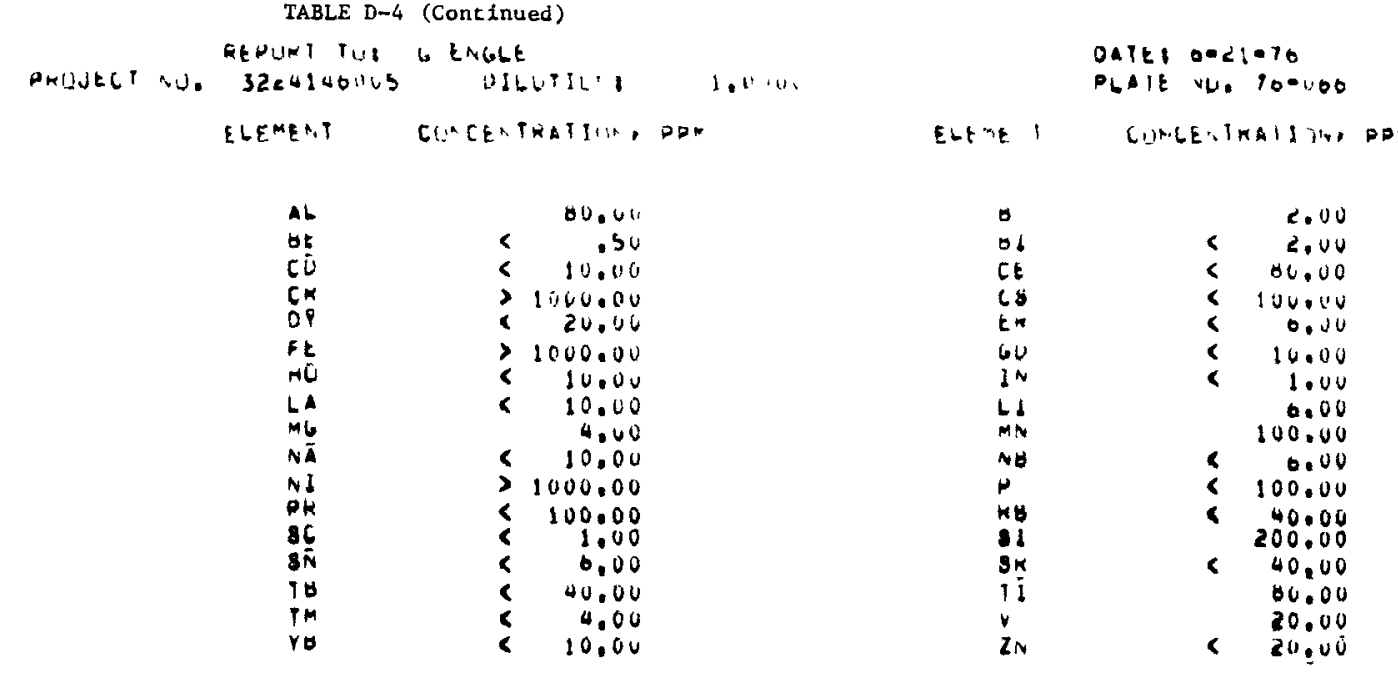

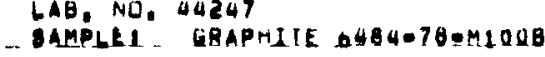

ELEMENT CONGENTRATIUN, PPM

PRUJECT REPURT IUI G ENGLE

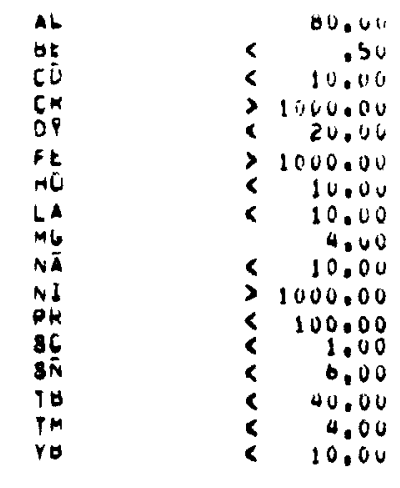

ELEMENT

CONCENTRATIUN, PPM

1.0000

\begin{tabular}{|c|c|c|}
\hline$A G$ & 6 & .50 \\
\hline CA & & $\begin{array}{r}20.00 \\
100.00\end{array}$ \\
\hline cu & C & 4.00 \\
\hline EU & \{ & $\begin{array}{r}1.00 \\
10.00\end{array}$ \\
\hline MF & c & 20.00 \\
\hline$k$ & 5 & 10.00 \\
\hline wH & $<$ & .50 \\
\hline MU & 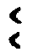 & 200000 \\
\hline $\begin{array}{l}P B \\
\text { SB }\end{array}$ & & $\begin{array}{r}200.00 \\
40.00\end{array}$ \\
\hline SM & 5 & 140.40 \\
\hline TA & 3 & $\begin{array}{l}0.00 \\
20.00\end{array}$ \\
\hline * & $<$ & 40.00 \\
\hline $2^{k}$ & & 10.00 \\
\hline
\end{tabular}

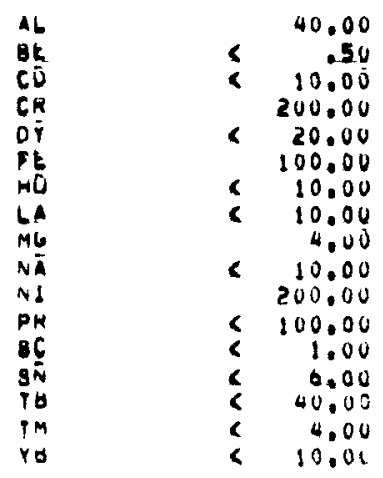

DAIES $0.21-76$

PLAIE NOA $10-426$

ELEMEAT

CONCENTHATIUN, POM

CONCENTAATION GASEU UN GRGIMAL SAMPLE BEFURE OILUTIIN WITH DILUENT

CMEANS GREATER THAN

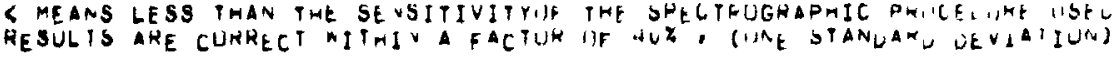

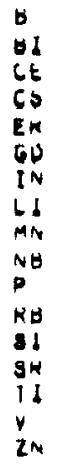

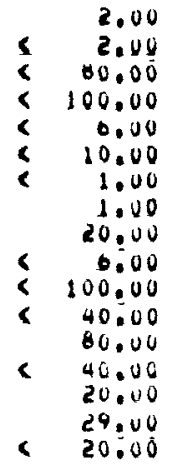




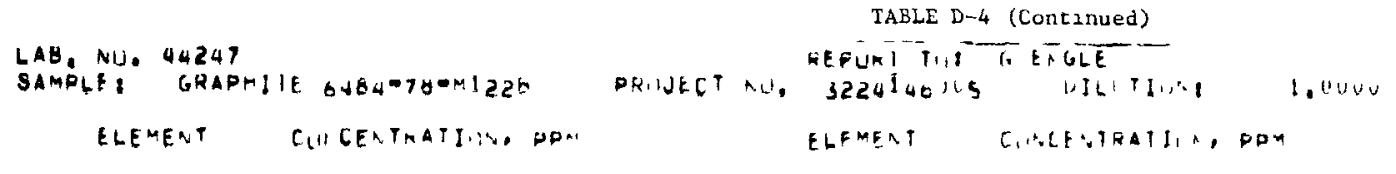

$A G$
$B A$
$C A$
$C O$
$C U$
$E U$
$H G$
$K$
$L U$
$M O$
$N O$
$P B$
$8 B$
$S M$
$T A$
$I L$
$W E$
$2 E$

$\underset{v}{1}$

$$
\begin{aligned}
& \begin{array}{r}
.50 \\
40.00
\end{array} \\
& 4.00 \\
& \begin{array}{r}
4.00 \\
\hline \quad 1.00
\end{array} \\
& \text { < } \quad 10.00 \\
& \begin{array}{l}
20.00 \\
10.00
\end{array} \\
& \begin{array}{r}
1.50 \\
<\quad 20.00 \\
\hline
\end{array} \\
& \begin{array}{r}
200.00 \\
<\quad 0.00
\end{array} \\
& <\quad 8.00 \\
& \left\{\begin{array}{r}
100.00 \\
40.00
\end{array}\right. \\
& \begin{array}{l}
20.00 \\
40.00
\end{array} \\
& .50
\end{aligned}
$$

\begin{tabular}{|c|c|c|}
\hline$\Delta L$ & & 100.04 \\
\hline$\forall E$ & $<$ & .50 \\
\hline & $<$ & 19.00 \\
\hline$C K$ & $<$ & 10.0 \\
\hline ur & $<$ & 20.0 \\
\hline $\mathrm{FE}$ & & 400.0 \\
\hline सİ & $<$ & 100 \\
\hline LA & i & 10.0 \\
\hline 46 & & 2.0 \\
\hline Nä & $<$ & 10.0 \\
\hline N1 & & 10.0 \\
\hline PK & 6 & 100.0 \\
\hline 86 & 5 & $\begin{array}{l}1.00 \\
6.00\end{array}$ \\
\hline $8 \bar{n}$ & 5 & $\begin{array}{r}6.00 \\
40,00\end{array}$ \\
\hline TS & z & $\begin{array}{r}40.0 \\
4.0\end{array}$ \\
\hline YB & $c$ & 10.00 \\
\hline
\end{tabular}

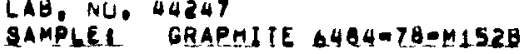

\begin{tabular}{|c|c|}
\hline & .50 \\
\hline & \\
\hline & \\
\hline & \\
\hline & \\
\hline & \\
\hline & \\
\hline & \\
\hline & \\
\hline & \\
\hline & \\
\hline & \\
\hline & \\
\hline & \\
\hline & $\begin{array}{l}40.00 \\
00.00\end{array}$ \\
\hline & $\begin{array}{l}4.00 \\
1.00\end{array}$ \\
\hline & 10.00 \\
\hline & 10.00 \\
\hline & \\
\hline & 200.00 \\
\hline & 0.00 \\
\hline & 100.64 \\
\hline & \\
\hline & \\
\hline
\end{tabular}

ELEMENT

CONCENTRATION, PPM

PROJEET NOA

RE POS

epuar tol

\begin{abstract}
ELEMENT
COACENTRATIUN, PPM
\end{abstract}

DILUTIONT.

1.0000

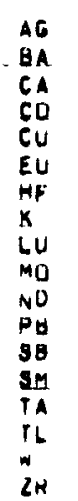

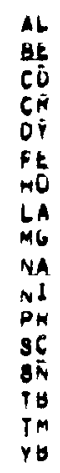

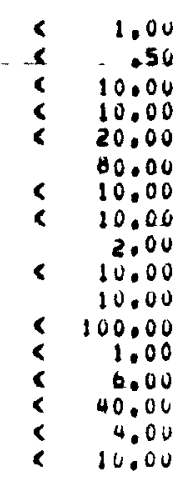

UIK: $0.21 \cdot 10$ LATE .u. 10-viog

CNLE THATLI D POM

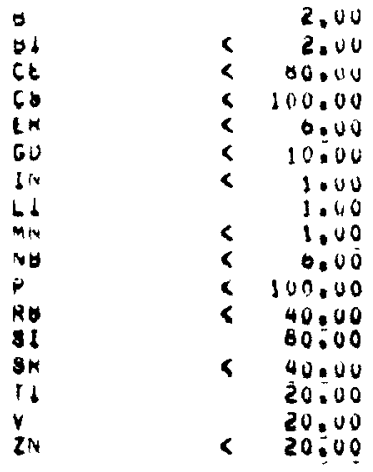

\section{DATE' 0021070}

CONGETHATION, PP

ELEMENT

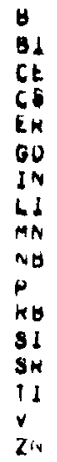

CONCENTKATION OASEO UN IJMGINAL SAMPLE GEFURE OILUTION MITH UILUENT

> MEANS GREATEK ITAN

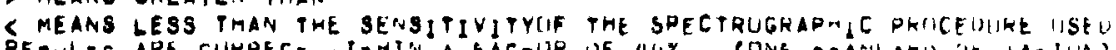

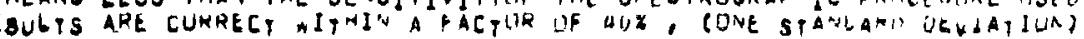

